\title{
Lysosomal and mitochondrial crosstalk: the role of lysosomal signaling on mitochondrial biogenesis and function
}

\author{
Dissertation \\ in partial fulfilment of the requirements \\ for the degree "Doctor of Philosophy (PhD)" \\ in the Neuroscience Program \\ at the Georg August University Göttingen, \\ Faculty of Biology
}

submitted by

King Faisal Yambire

born in

Bolgatanga, Ghana

Göttingen, September 2017 


\section{Members of the Thesis Advisory Committee}

\author{
Dr. Ira Milosevic \\ (Co-supervisor and first referee)
}

Prof. Dr. André Fischer (second referee)

Prof. Dr. Klaus-Armin Nave
Synaptic Vesicle Dynamics

European Neuroscience Institute Göttingen, Germany

Department of Psychiatry and Psychotherapy University Medical Center German Center for Neurodegenerative diseases Göttingen, Germany

Department of Neurogenetics Max Planck Institute for Experimental Medicine Göttingen, Germany

\section{Extended Thesis Committee members}

Dr. Nuno Raimundo

(Co-supervisor)

Prof. Dr. Steven Johnsen
Institute of Cellular Biochemistry

Department of Biochemistry

University Medical Center

Göttingen, Germany

Translational Cancer Research

University Medical Center

German Center for Molecular Biosciences

Göttingen, Germany 
"One thing I have learned in a long life: that all our science, measured against reality, is primitive and childlike and yet it is the most precious thing we have".

\section{ALBERT EINSTEIN}


Martha Y. Yambire, in memoriam 


\section{Affidavit}

I hereby declare that the dissertation, entitled 'Lysosomal and mitochondrial crosstalk: the role of lysosomal signaling on mitochondrial biogenesis and function', was written independently and with no other aids or sources than quoted.

King Faisal Yambire

Göttingen, September 2017 


\section{Table of Contents}

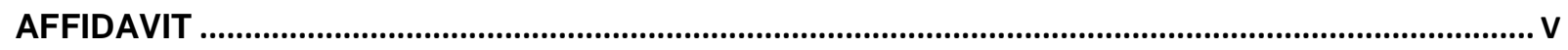

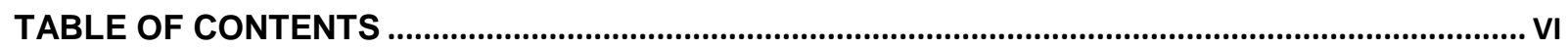

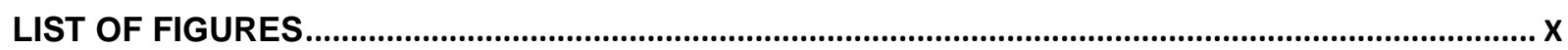

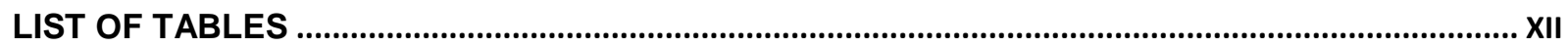

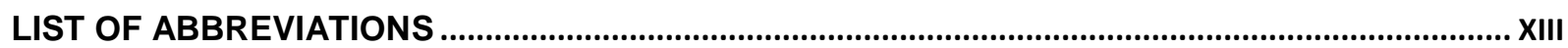

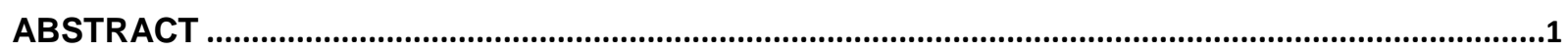

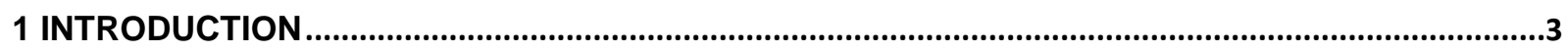

1.1 EVOLUTION OF THE EUKARYOTIC CELL: MEMBRANE-BOUND ORGANELLES …......................................... 3

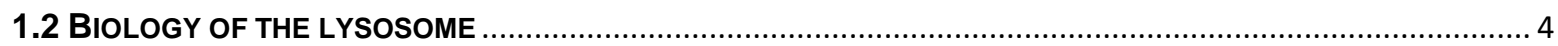

1.3 STRUCTURE, BIOGENESIS, AND FUNCTION OF THE LYSOSOME .................................................. 5

1.3.1 Structure of the lysosome

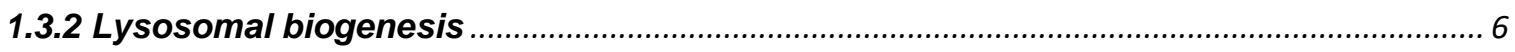

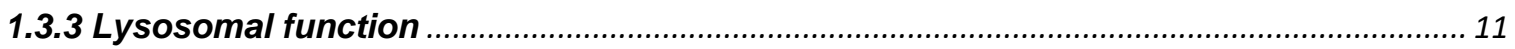

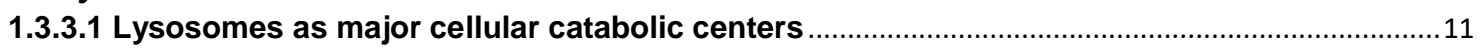

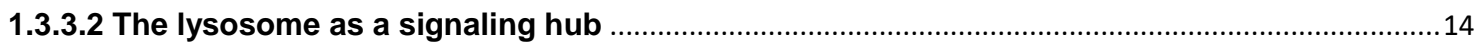

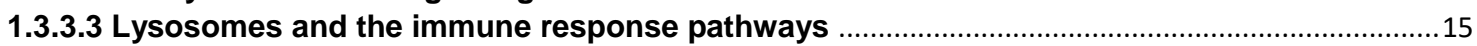

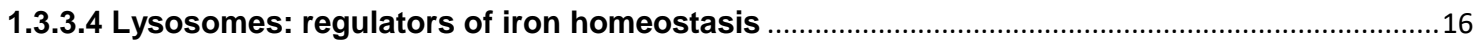

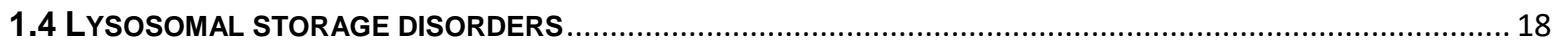

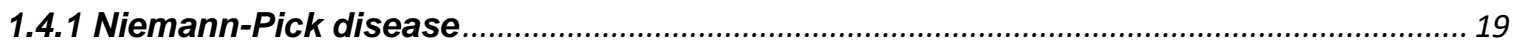

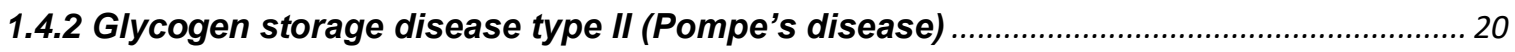

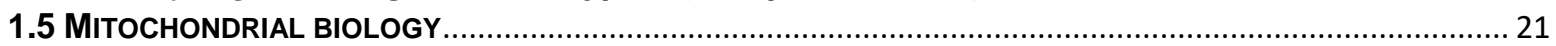

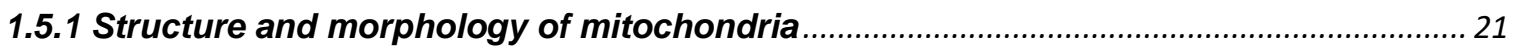

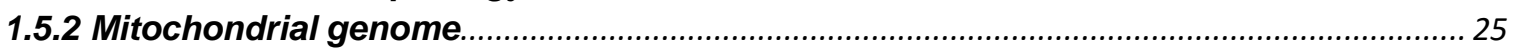

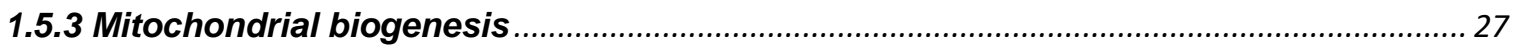

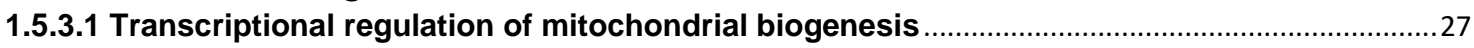

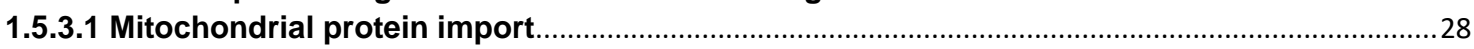

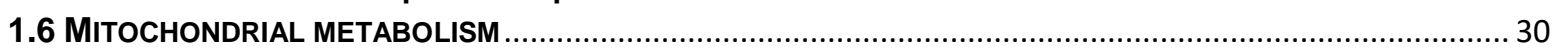

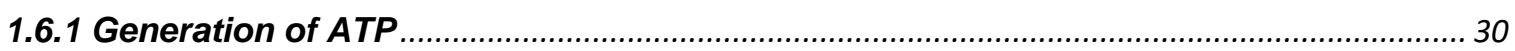

1.6.1.1 Glycolysis

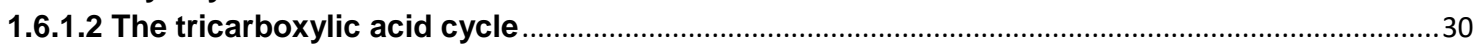

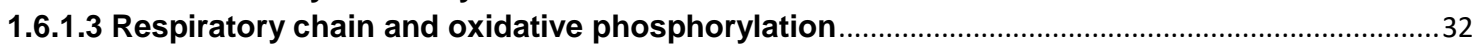

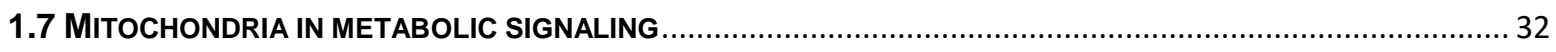

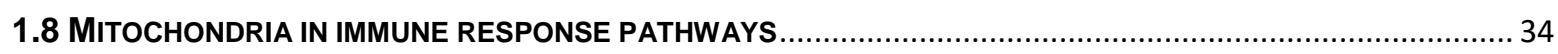

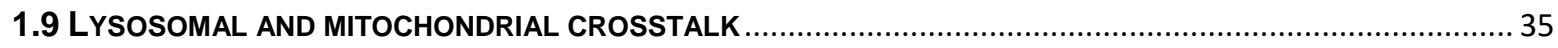

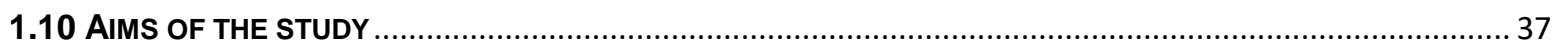

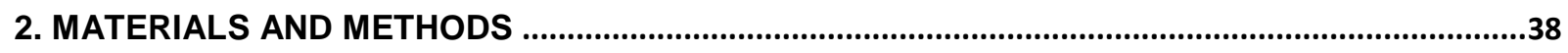

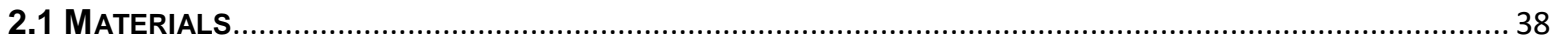

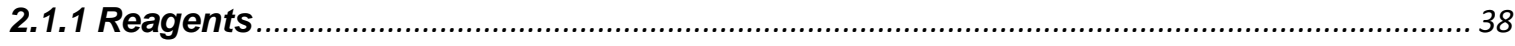

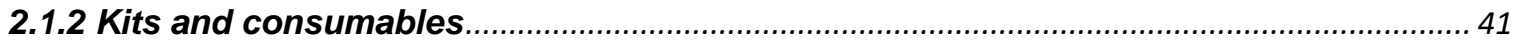

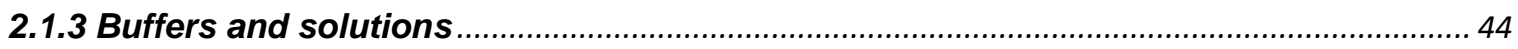

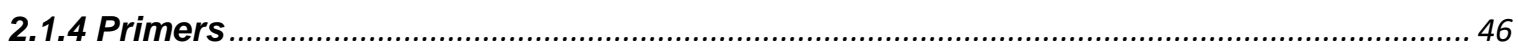

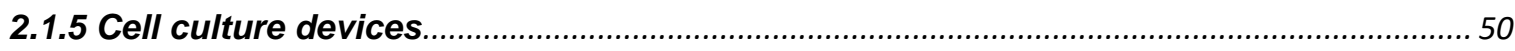

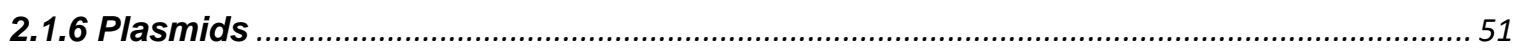

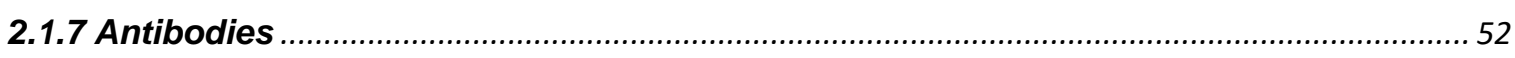




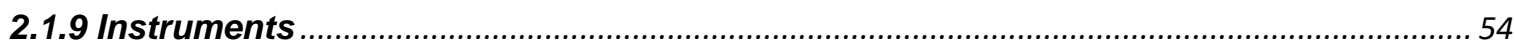

2.1.10 Software

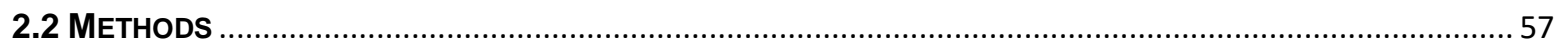

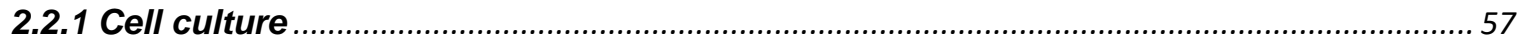

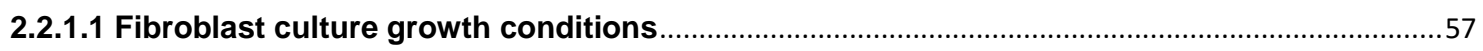

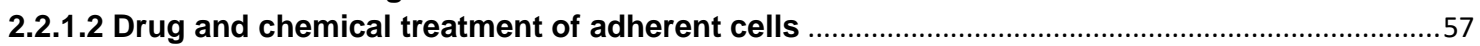

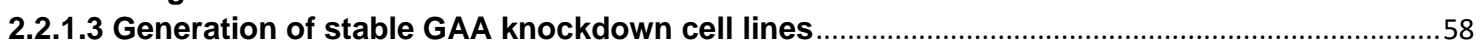

2.2.1.4 Transfection of dermal and mouse embryonic fibroblasts .....................................................58

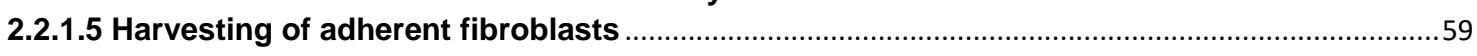

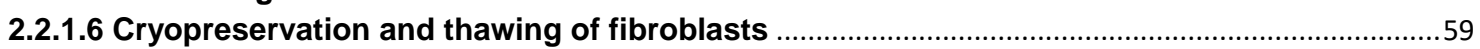

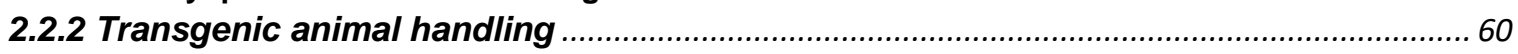

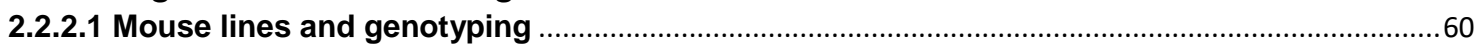

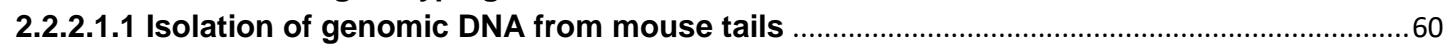

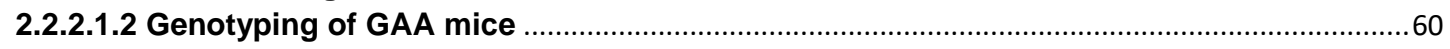

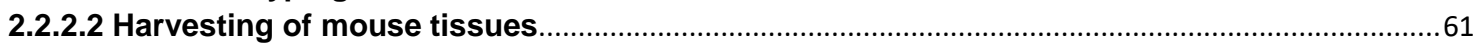

2.2.2.3 Perfusion and fixation of mouse tissues and immunohistochemistry ...................................62

2.2.2.4 Isolation of mitochondria from mouse tissue ...................................................................62

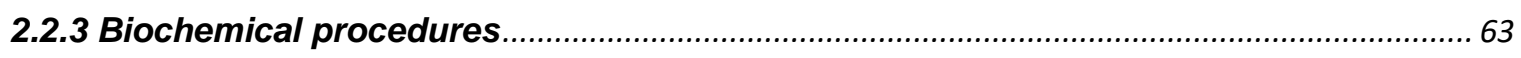

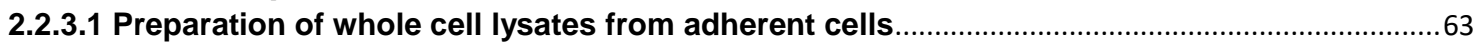

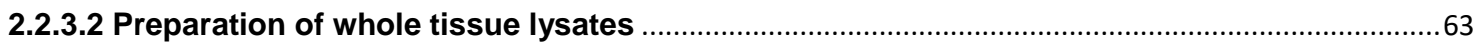

2.2.3.3 Protein concentration determination using Bradford assay .................................................64

2.2.3.4 Protein concentration determination using Pierce BCA assay ..............................................64

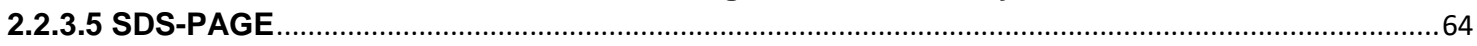

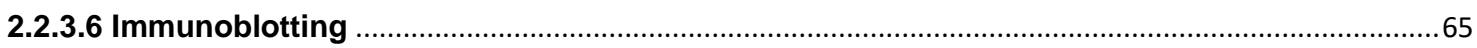

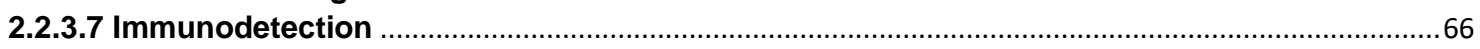

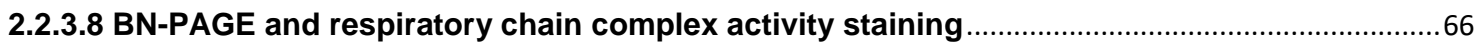

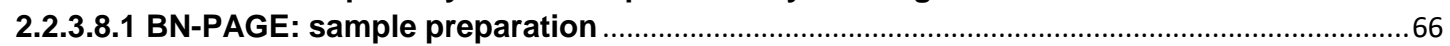

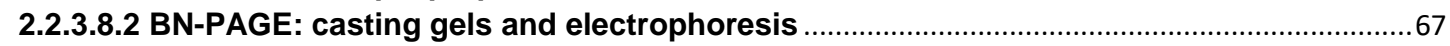

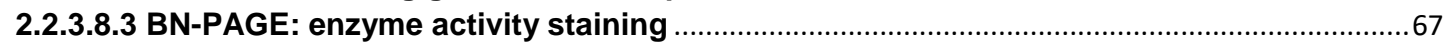

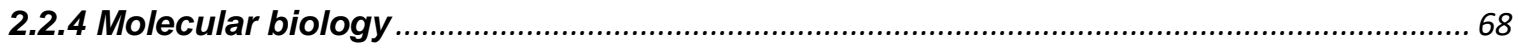

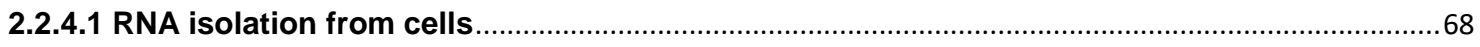

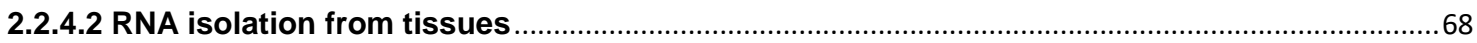

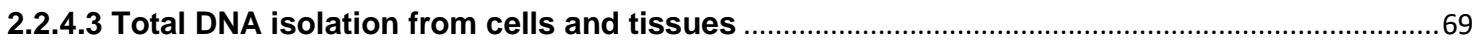

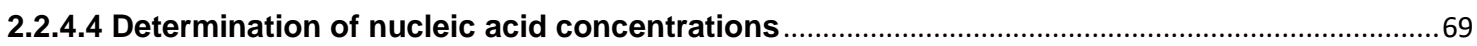

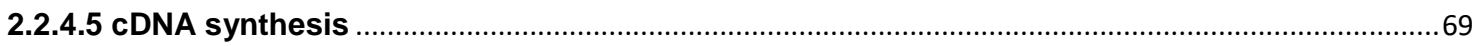

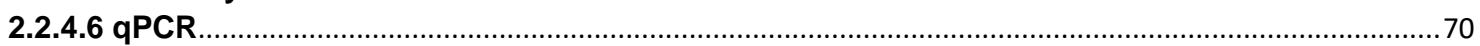

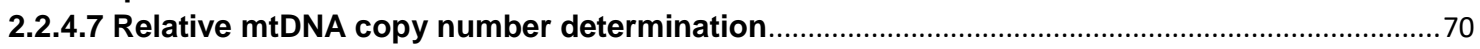

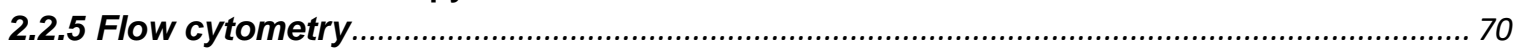

2.2.5.1 Apoptosis analyses by Annexin V and Propidium lodide staining assay ...............................71

2.2.5.2 Measurement of mitochondrial superoxide levels ...................................................................... 71

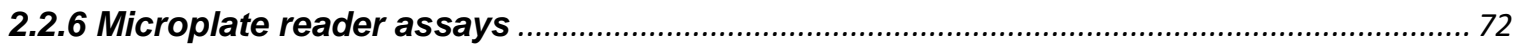

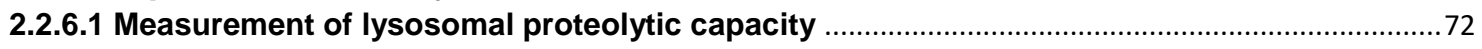

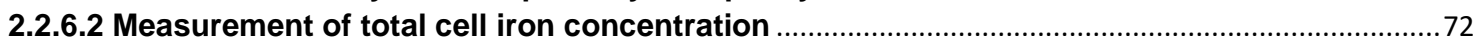

2.2.6.3 Protein concentration determination using Pierce BCA microassay .......................................73

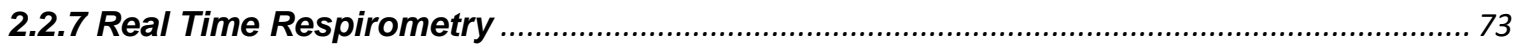

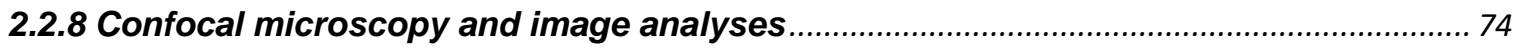

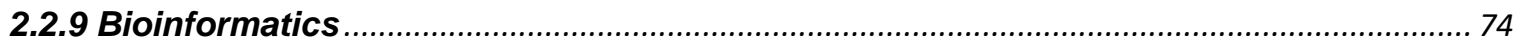

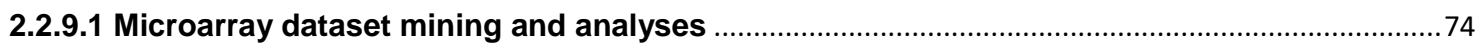

2.2.9.2 Pathway analyses and identification of transcriptional regulators ....................................... 75

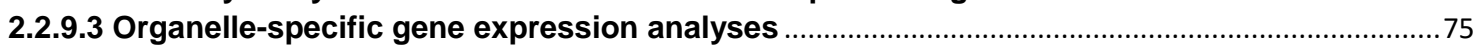

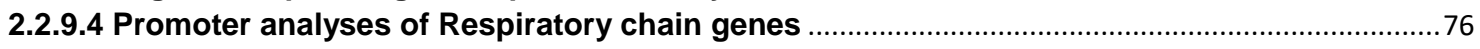

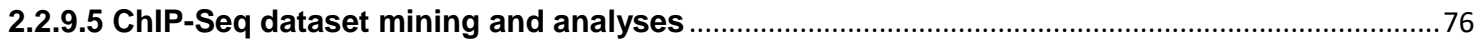

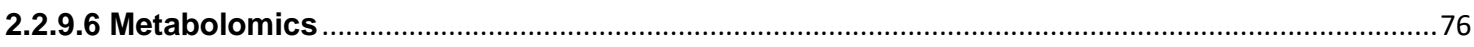




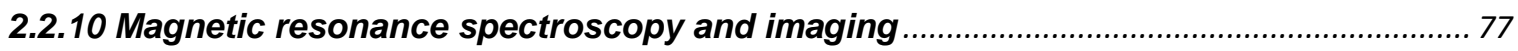

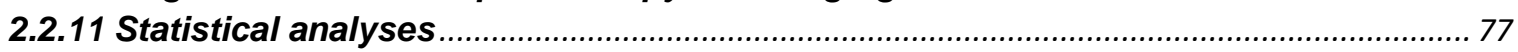

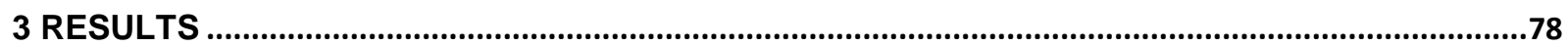

3.1 IMPAIRED SPHINGOSINE-1-PHOSPHATE SIGNALING IN NIEMANN-PICK DISEASE REPRESSES

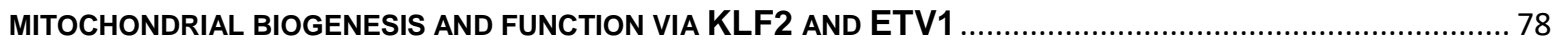

3.1.1 Lysosomal dysfunction in Niemann-Pick patient fibroblasts ......................................... 78

3.1.2 Mitochondrial function is compromised in NP fibroblasts............................................. 79

3.1.3 Repression of mitochondrial biogenesis in NP fibroblasts......................................... 80

3.1.4 Desipramine treatment impairs lysosomal function ...................................................... 82

3.1.5 Desipramine treatment represses mitochondrial biogenesis and function ................... 82

3.1.6 Transcriptomics reveal global downregulation of mitochondria-related genes in NPC1-

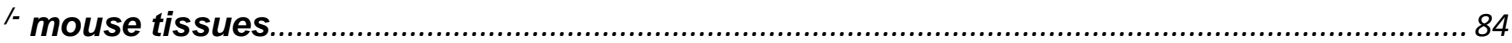

3.1.7 Reduced mitochondrial respiration in NPC1 ${ }^{-/}$MEFs................................................... 87

3.1.8 KLF2 and ETV1 mediate repression of mitochondrial biogenesis in Niemann-Pick

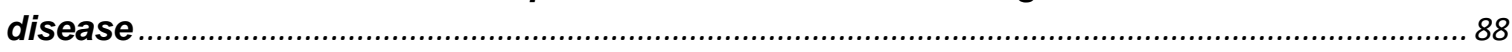

3.1.9 Mechanism of KLF2 and ETV1 regulation in Niemann-Pick disease ............................. 92

3.1.9.1 KLF2 activates ETV1 in an ERK-dependent manner ……......................................................93

3.1.9.2 Impaired sphingosine-1-phosphate signaling activates KLF2 ……...................................94

3.1.10 Activation of sphingosine 1-phosphate signaling restores mitochondrial function in

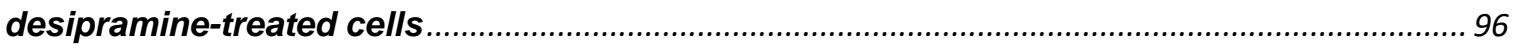

3.1.11 Induction of KLF2 and ETV1 in Niemann-Pick disease is cytoprotective.................... 96

3.2 IMPAIRED IRON HOMEOSTASIS DUE TO LYSOSOMAL MALFUNCTION CAUSES MTDNA DEPLETION, WHICH

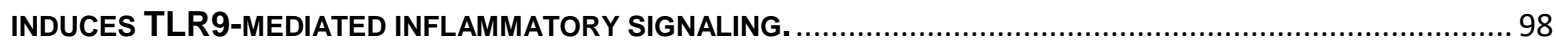

3.2.1 Cortical mitochondrial defects in Pompe's disease model ..........................................98

3.2.2 Progressive cortical mtDNA depletion in GAA ${ }^{-/}$mice ................................................. 100

3.2.3 Cardiac mitochondrial deficits in Pompe's disease ...................................................... 101

3.2.4 Loss of TFAM but not mtDNA depletion in GAA ${ }^{-/}$is dependent on mTORC1 ............. 103

3.2.5 Impaired nucleotide metabolism affects mtDNA levels.............................................. 105

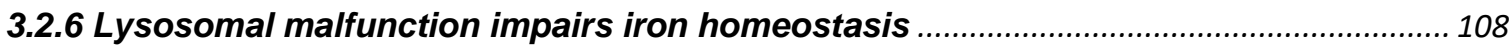

3.2.6 Iron deficiency causes mtDNA depletion ................................................................... 110

3.2.7 mtDNA defects due to iron deficiency trigger the innate immune response................ 112

3.2.8 Increased gliosis in the cortex of GAA ${ }^{-/-}$mice............................................................ 114

3.2.9 Iron supplementation ameliorates brain mtDNA defects and reduces innate immune

response in $\mathrm{GAA}^{-/-}$mice in vivo.

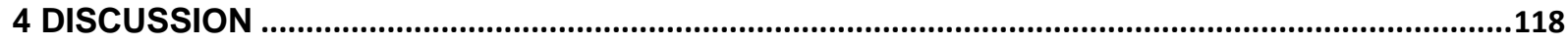

4.1 Defective lysosomal Signaling IN NIEMANN-PICK DISEASE REPRESSES MitochondRIAL

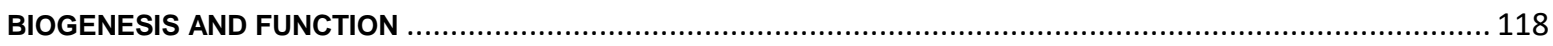

4.1.1 Mitochondrial biogenesis is repressed in Niemann-Pick disease .............................. 118

4.1.2 KLF2 and ETV1 mediate repression of mitochondrial biogenesis in Niemann-Pick

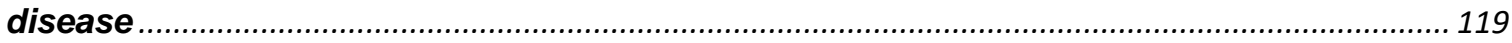

4.1.2 Mechanisms of KLF2 and ETV1 induction in Niemann-Pick disease .......................... 120

4.2 PERTURBED IRON HOMEOSTASIS LINKS LYSOSOMAL MALFUNCTION TO MTDNA DEPLETION AND

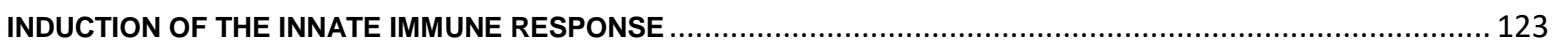

4.2.1 Mitochondrial genome defects in Pompe's disease ................................................... 123

4.2.2 Mechanisms of mtDNA depletion in Pompe's disease .................................................. 124

4.2.3 Lysosomal malfunction impairs iron homeostasis ...................................................... 125

4.2.5 Induction of innate immune response in $\mathrm{GAA}^{-/-}$mice .................................................. 127

4.2.6 Iron rescues $m$ tDNA defects and induced immune responses.................................. 128 


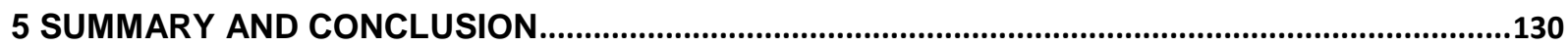

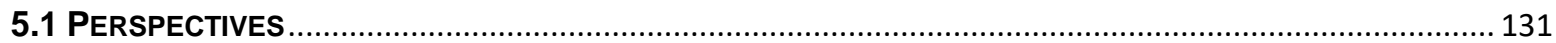

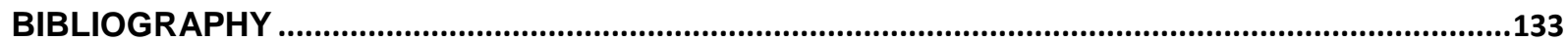

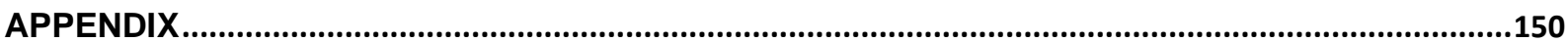

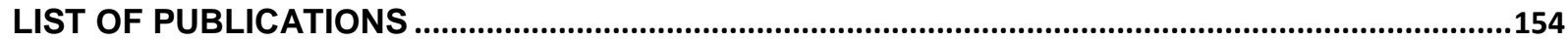

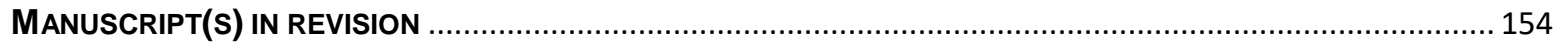

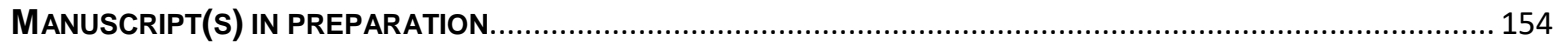

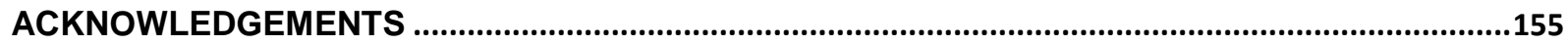

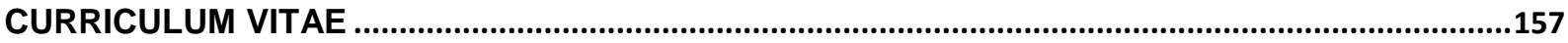




\section{List of figures}

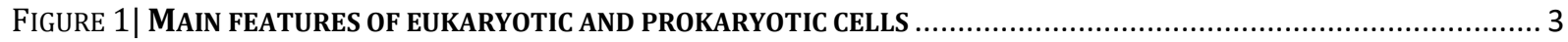

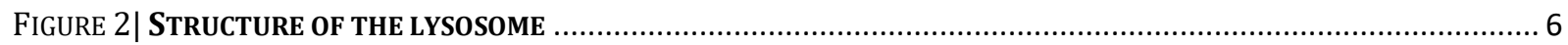

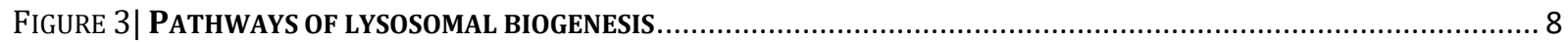

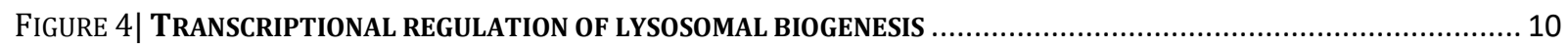

FIGURE 5| MACROAUTOPHAGY PATHWAY DEGRADES CELLULAR CONTENTS WITHIN LYSOSOMES................................. 13

FIGURE 6| MICROAUTOPHAGY AND CHAPERONE-MEDIATED AUTOPHAGY PATHWAYS MEDIATE DIRECT LYSOSOMAL

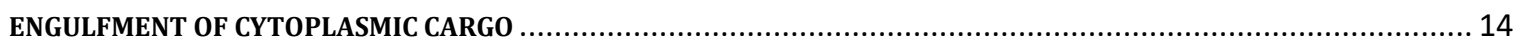

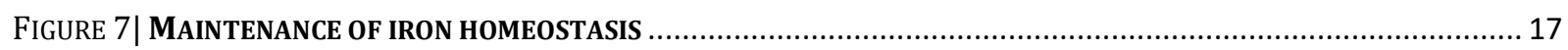

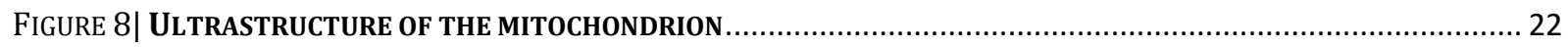

FIGURE 9| MITOCHONDRIAL NETWORK IN MOUSE EMBRYONIC FIBROBLASTS ……............................................... 24

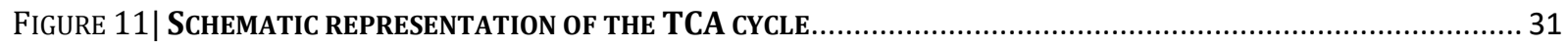

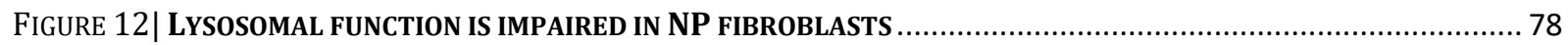

FIGURE 13| MITOCHONDRIAL MALFUNCTION IN NP FIBROBLASTS..................................................................... 79

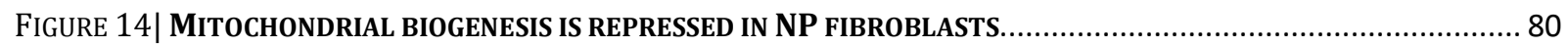

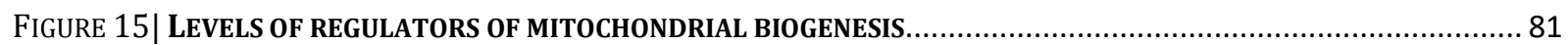

FIGURE 16| LYSOSOMAL FUNCTION IS IMPAIRED IN DESIPRAMINE-TREATED CELLS................................................ 82

FIGURE 17| DESIPRAMINE TREATMENT REPRESSES MITOCHONDRIAL BIOGENESIS AND FUNCTION .............................. 83

FIGURE 18| SCHEMATIC REPRESENTATION OF GENOMICS APPROACH FOR IN SILICO EXPERIMENTS ……...................... 84

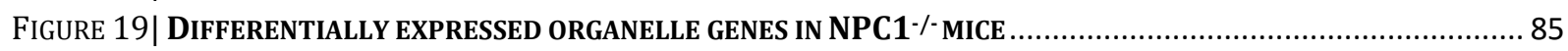

FIGURE 20| DISEASE PROGRESSION CHANGES IN MITOCHONDRIAL GENE EXPRESSION .............................................. 86

FIGURE 21| ORGANELLE-SPECIFIC GENE EXPRESSION CHANGES IN NPC1\% MICE …............................................. 87

FIGURE 22| MITOCHONDRIAL RESPIRATION IS REDUCED IN NPC1 $\%$ MEFS …....................................................... 88

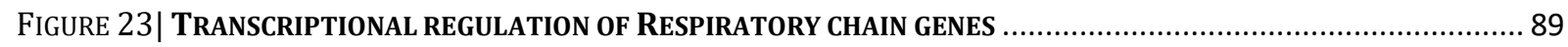

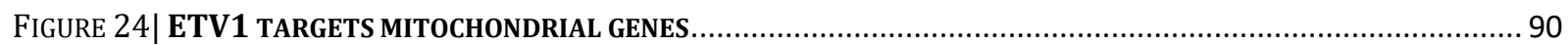

FIGURE 25| KLF2 AND ETV1 REPRESS MITOCHONDRIAL BIOGENESIS AND FUNCTION.......................................... 91

FIGURE 26| AUTOPHAGY DEFECTS IN NP ARE INDEPENDENT OF KLF2 AND ETV1 …........................................... 92

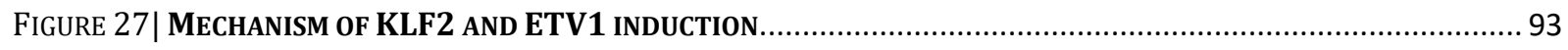

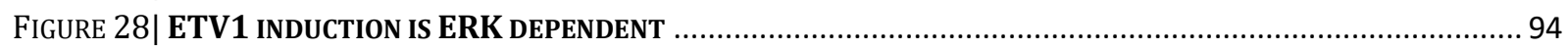

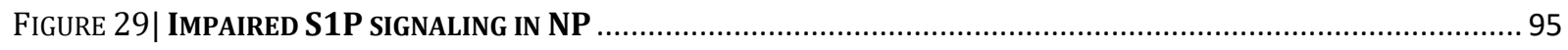

FIGURE 30| S1PR1 SIGNALING RESCUES MITOCHONDRIAL FUNCTION IN ASM INHIBITED CELLS............................... 96

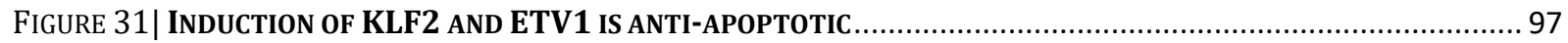

FIGURE 32| REPRESSED MITOCHONDRIAL BIOGENESIS IN GAA / CORTEX ...................................................... 99

FIGURE 33| REDUCED TRANSCRIPTIONAL REGULATION OF MITOCHONDRIAL BIOGENESIS .......................................... 100

FIGURE 34| PROGRESSIVE MTDNA COPY NUMBER DEPLETION IN GAA \% CORTEX ................................................. 100

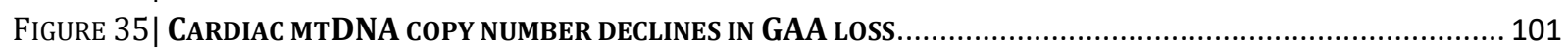

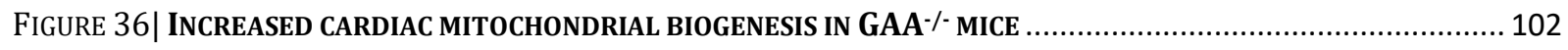

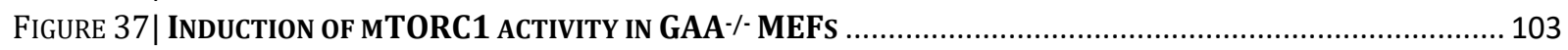

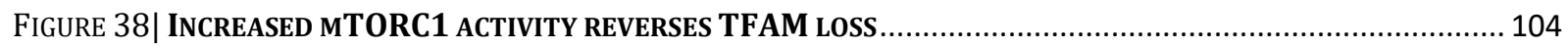

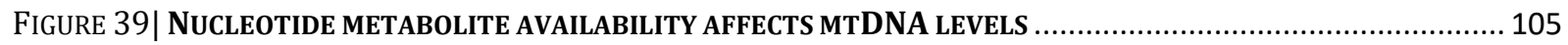

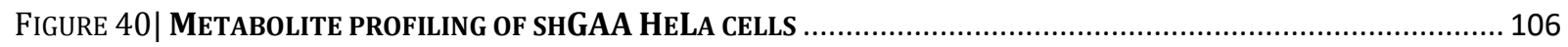

FIGURE 41| ENHANCING NUCLEOTIDE SYNTHESIS RESCUES MTDNA DEPLETION ................................................ 108

FIGURE 42| DECREASED COMPLEX IV ACTIVITY IN GAA \% CORTICAL MITOCHONDRIA ........................................... 109

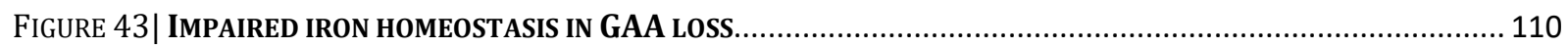

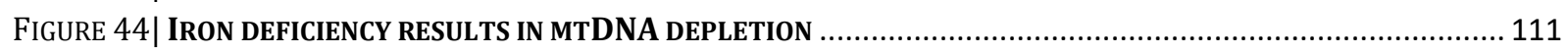

FIGURE 45 | IRON SUPPLEMENTATION RESCUES MTDNA COPY NUMBER DEPLETION ............................................. 112

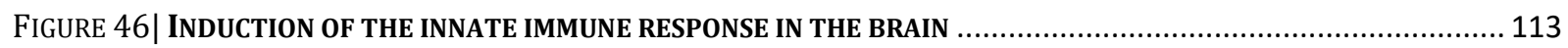

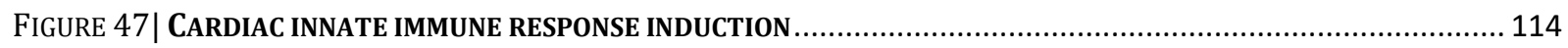

FIGURE 48| GLIAL CELL PROLIFERATION IN THE CORTEX OF GAA $\%$ MICE........................................................ 115 
FIGURE 49| IRON SUPPLEMENTATION RESCUES MTDNA DEFECTS AND DOWN-REGULATES INNATE IMMUNE ACTIVATION

FIGURE 50| MODEL OF LYSOSOMAL AND MITOCHONDRIAL CROSSTALK IN NIEMANN-PICK DISEASE ........................ 122

FIGURE 51| MECHANISM OF LYSOSOMAL-MITOCHONDRIAL CROSSTALK IN POMPE'S DISEASE .................................. 129

FIGURE 52| EXPRESSION LEVELS OF ETS TRANSCRIPTION FACTORS IN NP PATIENT CELLS ................................. 150

FIGURE 53| KLF2, ETV1 AND TFAM PROTEIN LEVELS IN DESIPRAMINE-TREATED CELLS .................................. 150

FIGURE 54| ENHANCED S1P SIGNALING INDUCES MITOCHONDRIAL GENE EXPRESSION..................................... 151

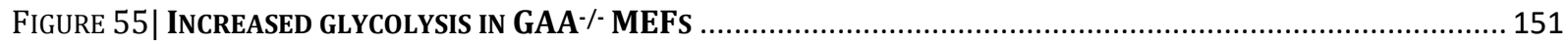

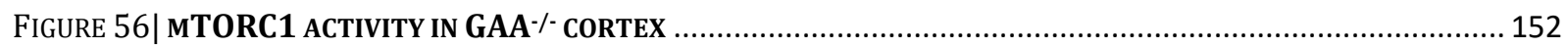

FIGURE 57| LONP1 LEVELS ARE PARTLY DEPENDENT ON MTORC1 ACTIVITY IN GAA $/$ - MEFS ........................... 152

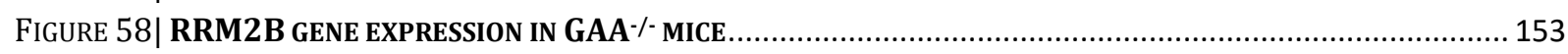




\section{List of tables}

TABLE 1: LIST OF REAGENTS AND THEIR SUPPLIERS AND CATALOG NUMBERS............................................ 38

TABLE 2: LIST OF KITS AND CONSUMABLESABLES USED IN THE STUDY ...................................................... 41

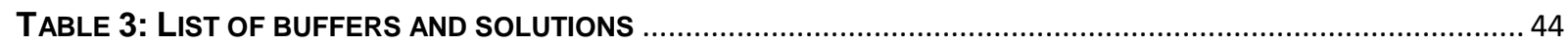

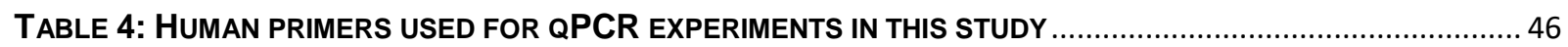

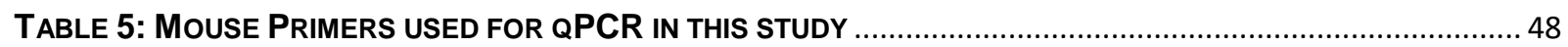

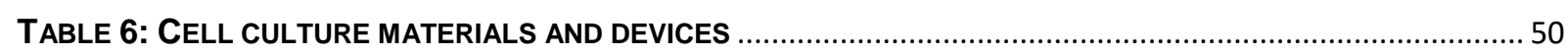

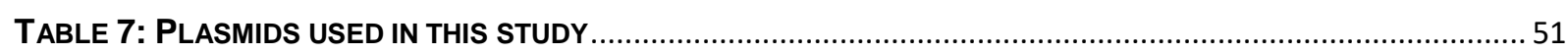

TABLE 8: PRIMARY ANTIBODIES FOR IMMUNOBLOTTING AND IMMUNOCYTOCHEMISTRY ................................... 52

TABLE 9: SECONDARY ANTIBODIES FOR IMMUNOBLOTTING AND IMMUNOCYTOCHEMISTRY ............................. 54

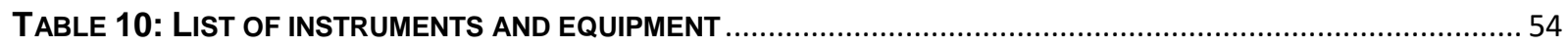

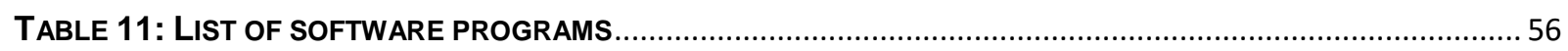

TABLE 12: SEEDING DENSITY AND APPLICATION FOR CELL CULTURE EXPERIMENTS .................................... 57

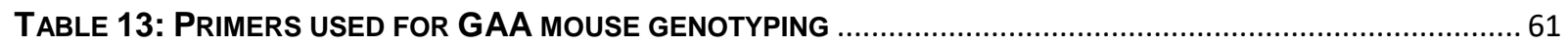

TABLE 14: SOURCE OF ORGANELLE-SPECIFIC PROTEOME DATABASE …….................................................. 75

TABLE 15: TRANSCRIPTION FACTORS WITH ENRICHED CIS-ELEMENTS IN RC GENE PROMOTERS .................... 89

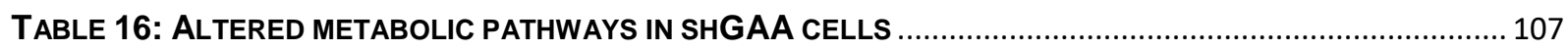

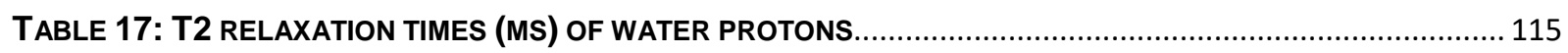




\section{List of abbreviations}

$A D$

ADP

ALS

AMP

AMPK

APS

ASM

ATP

$\mathrm{BN}$

BSA

CCCP

cDNA

ChIP-Seq

CMA

CNS

CoA

DAPI

${ }_{\text {dd }} \mathrm{H}_{2} \mathrm{O}$

DEG

DMEM

DMSO

DNA

dNTPs

EBSS

EDTA

ER

FAD

$\mathrm{FADH}_{2}$

FBS

FCCP

GAA

gDNA

HEPES
Alzheimer's disease

Adenosine diphosphate

Amyotrophic lateral sclerosis

Adenosine monophosphate

AMP-activated protein kinase

Ammonium persulfate

Acid sphingomyelinase

Adenosine triphosphate

Blue Native

Bovine serum albumin

Carbonyl cyanide-m-chlorophenyl hydrazine

Complementary DNA

Chromatin immunoprecipitation sequencing

Chaperone-mediated autophagy

Central nervous system

Coenzyme A

4',6-diamidino-2-phenylindole

Double distilled water

Differentially expressed genes

Dulbecco's modified eagle's medium

Dimethyl sulfoxide

Deoxyribonucleic acid

Deoxyribonucleotide triphosphates

Earl's balanced salt solution

Ethylenediaminetetraacetic acid

Endoplasmic reticulum

Flavin adenine dinucleotide

Reduced Flavin adenine dinucleotide

Fetal bovine serum

Carbonyl cyanide-p-trifluoromethoxyphenol hydrazone

Acid alpha-glucosidase

Genomic DNA

4-(2-hydroxyethyl)-1-piperazineethanesulfonic acid 


$\begin{array}{ll}\text { HRP } & \text { Horseradish peroxidase } \\ \text { IBM } & \text { Inner boundary membrane } \\ \text { IMM } & \text { Inner mitochondrial membrane } \\ \text { LSDs } & \text { Lysosomal storage disorders } \\ \text { MEFs } & \text { Murine/mouse embryonic fibroblasts } \\ \text { MHC } & \text { Major histocompatibility complex } \\ \text { mRNA } & \text { Messenger RNA } \\ \text { mtDNA } & \text { Mitochondrial DNA } \\ \text { mTORC1 } & \text { mammalian/mechanistic Target of Rapamycin } \\ \text { NADH } & \text { Reduced Nicotinamide adenine dinucleotide } \\ \text { NMDA } & \text { N-methyl-D-aspartate } \\ \text { NP } & \text { Niemann-Pick disease } \\ \text { OCR } & \text { Oxygen consumption rate } \\ \text { OMM } & \text { Outer mitochondrial membrane } \\ \text { OXPHOS } & \text { Oxidative phosphorylation } \\ \text { PAGE } & \text { Polyacrylamide gel electrophoresis } \\ \text { PBS } & \text { Phosphate buffered saline } \\ \text { PCR } & \text { Polymerase chain reaction } \\ \text { PD } & \text { Parkinson's disease } \\ \text { PFA } & \text { Paraformaldehyde } \\ \text { PMSF } & \text { Phenylmethylsulfonyl fluoride } \\ \text { PVDF } & \text { Polyvinylidene fluoride } \\ \text { qPCR } & \text { Quantitative real time PCR } \\ \text { RC } & \text { Respiratory chain } \\ \text { RNA } & \text { Ribonucleic acid } \\ \text { ROS } & \text { Reactive oxygen species } \\ \text { rRNA } & \text { Ribosomal RNA } \\ \text { RT } & \text { Room temperature } \\ \text { S1P } & \text { Sphingosine-1-phosphate } \\ \text { SDS } & \text { Sodium dodecyl sulfate } \\ \text { shRNA } & \text { Short hairpin RNA } \\ \text { SiRNA } & \text { Short interfering RNA } \\ \text { TBST } & \text { Triethylenediamine } \\ \text { TEMED } & \end{array}$


TIM

TOM

tRNA

UMP
Translocase of the inner membrane

Translocase of the outer membrane

Transfer RNA

Uridine monophosphate 


\section{Abstract}

Defects in metabolism result in various diseases, with neurodegeneration being one of the most prevalent consequences. Mitochondria and lysosomes are long known to be fundamental for cellular metabolism. In addition to being energy 'factories', mitochondria are key cellular signaling platforms, contributing to cellular stress responses like autophagy, apoptosis or cell proliferation. Lysosomes have evolved beyond their 'waste management' roles and are now understood to coordinate major processes such as autophagy and nutrient sensing. Although for many years, these organelles were seen as independent functional entities in the cell, recent evidence suggests the existence of functional interdependent networks between lysosomes and mitochondria. In this study, we elucidate mechanisms beyond autophagy of crosstalk between lysosomes and mitochondria, and show that lysosomal defects affect mitochondrial function.

To identify mechanisms regulating lysosomal and mitochondrial crosstalk, we employed two distinct models of lysosomal storage disorders (LSDs): Niemann-Pick disease and Pompe's disease. We evaluated the effects of chronic lysosomal malfunction in these models on mitochondrial fitness and function.

We showed in patient cells of Niemann-Pick disease that impaired S1P signaling engages transcriptional programs, via KLF2 and ETV1, to repress mitochondrial biogenesis and function. In support of these findings, in silico analyses on microarray datasets of brain and liver samples of $\mathrm{NPC}^{-/}$mice confirmed the repression of mitochondrial biogenesis and the induction of KLF2 and ETV1. Interestingly, mechanisms of KLF2 and ETV1 downregulation, including siRNA-mediated silencing and enhanced S1P signaling, are sufficient to promote mitochondrial biogenesis in Niemann-Pick disease. These findings uncover the involvement of a transcriptional network in the regulation of lysosomal and mitochondrial crosstalk and the therapeutic potential of modulating S1P signaling in Niemann-Pick disease.

In the acid alpha-glucosidase (GAA) knockout mouse model, a model for the human Pompe's disease, we showed extensive mtDNA defects in various tissues and cells. Interestingly, loss of mtDNA was independent of mitochondrial biogenesis. Rather, we demonstrated that mtDNA depletion was dependent on reduced nucleotide bioavailability. Strikingly, mtDNA depletion could be reversed by enhanced nucleotide biosynthesis through folate supplementation. Furthermore, this study demonstrated that impaired iron homeostasis, which affected the activity of iron-containing proteins including respiratory chain complex IV and ribonucleotide 
reductases among others, was culpable for reduced nucleotide bioavailability and mtDNA depletion. In addition, mtDNA defects were associated with induction of the innate immune response via TLR9-mediated signaling in $\mathrm{GAA}^{-/-}$mouse tissues, which culminates in gliosis in the cortex. Strikingly, iron supplementation reverses mtDNA defects in MEFs and in the cortex of GAA knockout mice and dampens pro-inflammatory signaling in the cortex.

Altogether, the findings of this study demonstrate that lysosomal malfunction has detrimental consequences for mitochondrial fitness and function, both in vitro and in vivo, and elucidate novel mechanisms of lysosomal and mitochondrial interplay. In addition, this study shows that the mechanisms of organelle crosstalk could provide therapeutic avenues for LSDs and even for neurodegenerative diseases (e.g., Parkinson's disease). 


\section{Introduction}

\subsection{Evolution of the eukaryotic cell: membrane-bound organelles}

Cellular efficiency underlay the evolutionary success of eukaryotes which was based on the symbiotic acquisition of subcellular compartments thereby enabling essential functions in metabolism and signaling (Douglas, 2014). These subcellular compartments were restricted to the mediation of processes involved in energy production. As such, eukaryotic cells unequivocally became larger without compromising energy production (Figure 1A). Bacterial cells, on the other hand, which retained energy production machinery in the cell membrane, are restricted to relatively small sizes (Figure 1B) although they can attain larger cell sizes through a variety of adaptations (Lane and Martin, 2010; Schulz and Jørgensen, 2001). Furthermore, the membranes of these subcellular compartments provided platforms for several cellular processes while restricting different metabolic processes in separate compartments.

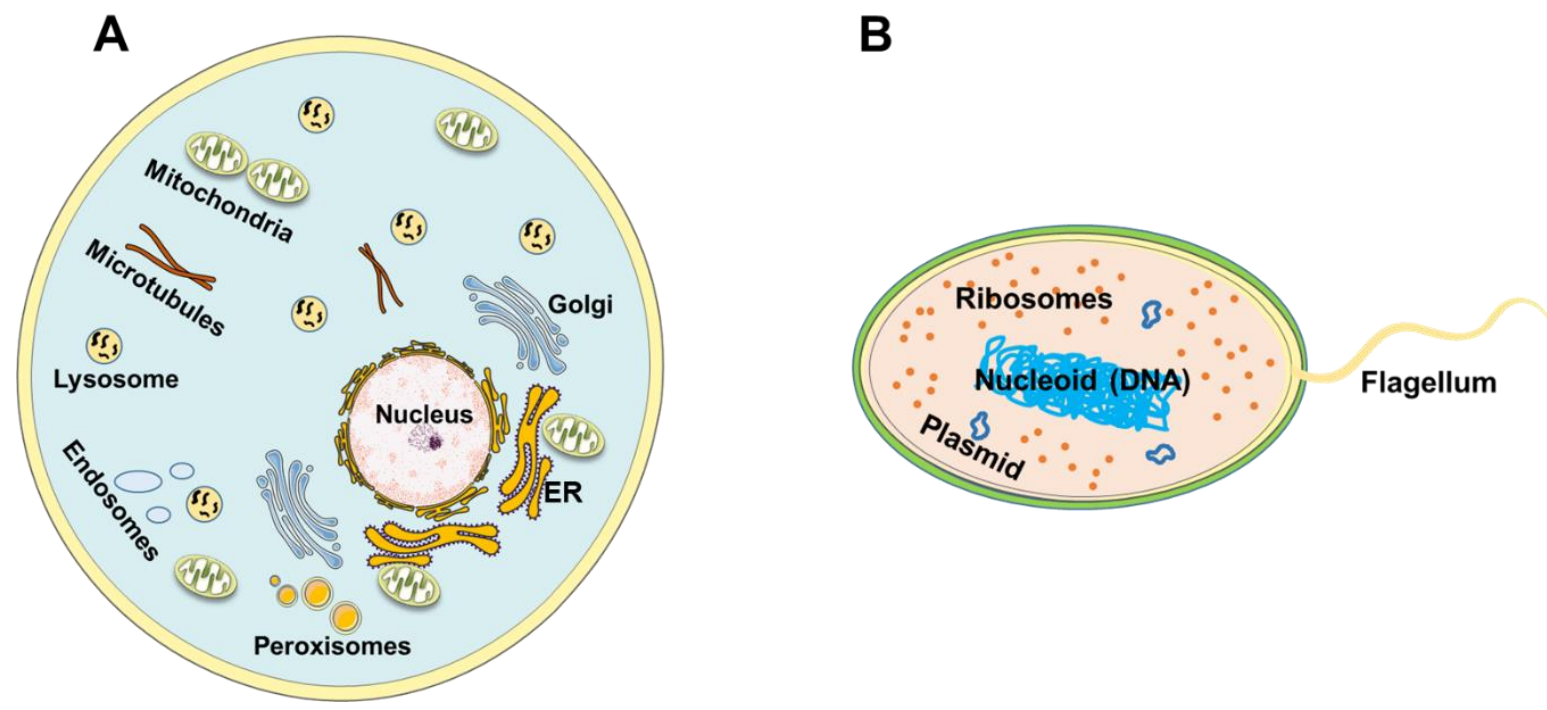

Figure 1| Main features of eukaryotic and prokaryotic cells -A A schematic representation of a simple eukaryotic cell, which is bound by a cell membrane showing intracellular components. Phototrophic eukaryotes contain plastids, which are not indicated. B A hypothetical flagellated prokaryote bound by a cell wall enclosing a cell membrane and defined by the lack of intracellular membrane-bound compartments. (original illustration, KF Yambire) 
For instance, lysosomes (or vacuoles) evolved in eukaryotes to assume most of the catabolic processes in the cell while mitochondria retained the energy production machinery of the cell. Undeniably, the acquisition of some of these organelles defined the evolution of eukaryotes, with the increased cellular efficiency shaping evolutionary diversification. It is unimaginable how specialized cells like myocytes and neurons would have been acquired without the dimension of lifeform ushered in by subcellular organelles.

It is widely accepted that eukaryotes of today evolved from an ancestor that already possessed a mitochondrion (Müller et al., 2012), the acquisition of which underlay the important events in the path to evolutionary success and diversification. In addition, a widely accepted mechanism of how the mitochondrion was acquired is solely based on phagocytosis. This is further supported by evidence of the existence of basic phagocytic and endocytic machinery prior to the endosymbiotic acquisition of the mitochondrion (Poole et al., 2014) suggesting an ancient interaction between the mitochondrion and the primitive lysosome. This study, which seeks to characterize such crosstalk between lysosomes and mitochondria, will also deepen our understanding of these two subcellular compartments in the succeeding sections.

\subsection{Biology of the lysosome}

In the early 1950s, while characterizing the role of glucose 6 -phosphatase in the mechanism of insulin action by tissue fractionation studies, Christian de Duve came across acid phosphatase-containing cytoplasmic granules which were entirely distinct from microsomal and mitochondrial fractions. These cytoplasmic granules, later termed lysosomes, were found to be rich in hydrolytic enzymes (De Duve et al., 1955). More than 50 of such acid hydrolytic enzymes have been discovered, making the lysosome the primary catabolic sub-compartment of the cell, where cellular components are broken down and recycled. These acid hydrolases are known to function optimally at acidic lysosomal luminal $\mathrm{pH}$ ranging between 4.5 and 5.0. The highly acidic lumen of the lysosome is maintained by the vacuolar $\mathrm{H}^{+}$ATPase (v-ATPase), which pumps protons into the lumen while providing the gradient for the counter flux of other ions like $\mathrm{Na}^{+}, \mathrm{K}^{+}$, and $\mathrm{Cl}^{-}$(Forgac, 2007; Mindell, 2012). The degradative role of the lysosome ultimately depends on the cargo that is delivered to it, of which multiple pathways of cargo delivery have been described. Endocytosis, including the primitive phagocytosis and micropinocytosis, deliver cargo from the extracellular space to the lysosome while the self-catabolic pathway of autophagy mediates the delivery of subcellular macromolecules or whole compartments sequestered in autophagosomes to the lysosome (Di Fiore and von Zastrow, 2014; Mizushima 
and Komatsu, 2011). Given the essential role of lysosomes in the maintenance of metabolic homeostasis, it is not surprising that gene mutations that affect key lysosomal components lead to a heterogeneous group of heritable disorders known as lysosomal storage disorders, which are often associated with neurodegenerative phenotypes (Platt et al., 2012; Sambri et al., 2017).

\subsection{Structure, biogenesis, and function of the lysosome}

\subsubsection{Structure of the lysosome}

Lysosomes are membrane-bound compartments of the eukaryotic cell, which contain several hydrolytic enzymes, thereby making them sites of intracellular digestion of macromolecules. As acid hydrolases, these enzymes require an acidic environment for their activation by proteolytic processing and for optimal activity. Although given this arrangement, lysosomal hydrolases are virtually inactive in the cytosol with $\mathrm{pH}$ of about 7.2, lysosomes are delimited by a phospholipid bilayer about 7-10nm thick, whose components allow for luminal acidification. Morphologically, lysosomes are a heterogeneous group of organelles with diverse shapes, sizes and numbers in a species-, cell type- and context-dependent manner. Several transmembrane proteins span the length of the lysosomal membrane, the most abundant of which are the lysosomalassociated membrane protein (LAMP) 1 and LAMP2, the lysosomal integral protein, LIMP2 and tetraspanin, CD63 (Perera and Zoncu, 2016; Saftig and Klumperman, 2009) as illustrated in Figure 2. These transmembrane proteins are unusually highly glycosylated on the luminal side, thus forming a glycocalyx, which serves to protect against self-digestion of the limiting lysosomal membrane. In addition to the v-ATPase which maintains the luminal $\mathrm{pH}$, several other transmembrane proteins, although less abundant, span the entire length of the lysosomal membrane and mediate processes which include transport of digestive end-products such as amino acids, sugars, lipids and nucleotides into the cytosol, and membrane fusion (Saftig and Klumperman, 2009). 


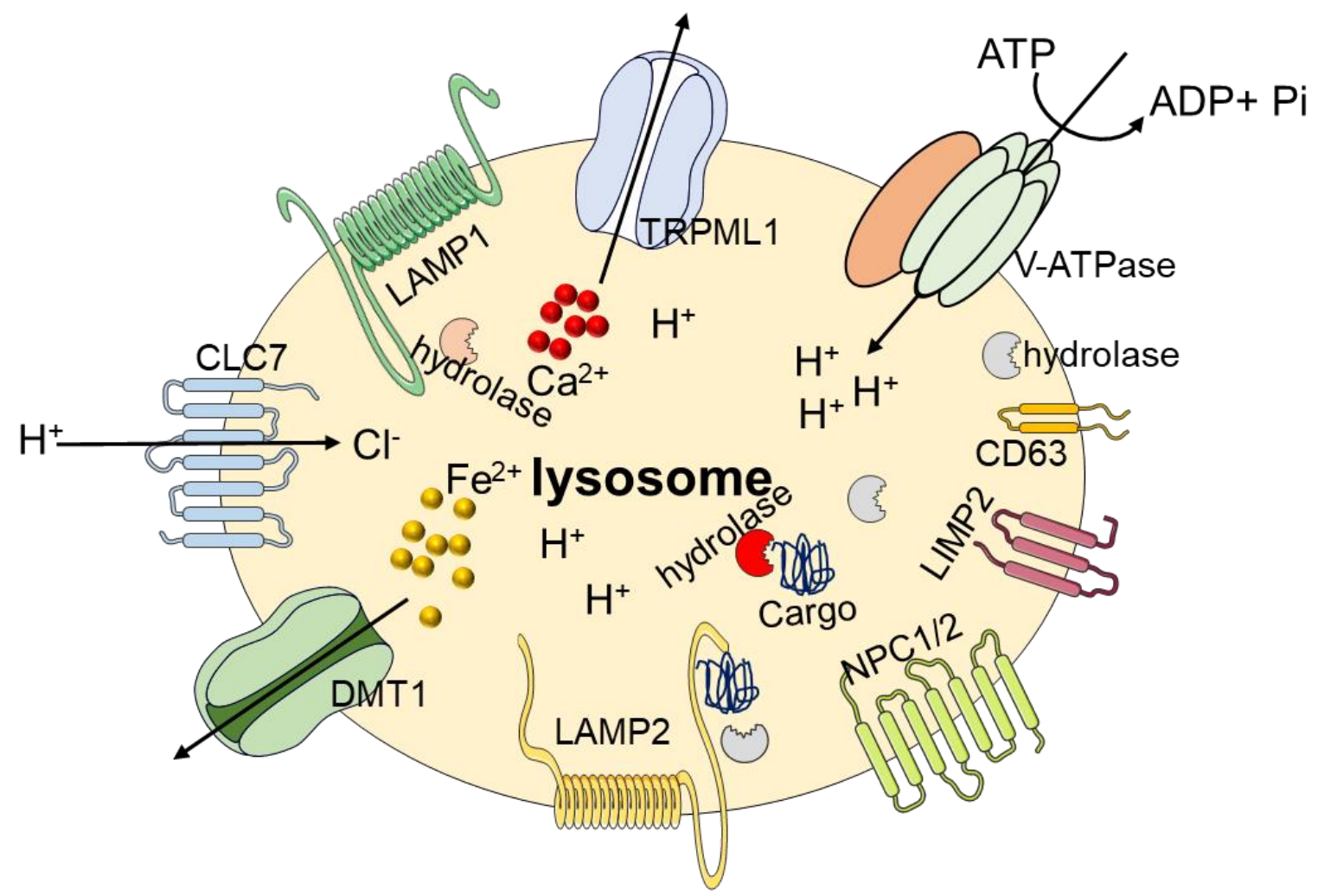

Figure 2| Structure of the lysosome - Lysosomes are membrane-bound organelles with several transmembrane proteins, some of which, LAMP1 and LAMP2 are indicated. The vATPases, which ensure luminal acidification, are also displayed. Different types of ion channels and nutrient exporters span the lysosomal membrane. The lumen contains hydrolases which degrade lysosomal cargo. (original illustration, KF Yambire)

\subsubsection{Lysosomal biogenesis}

Lysosomes arise from the integration of endocytic vesicles and those from the biosynthetic pathway of the cell through the trans-Golgi network. After budding from the plasma membrane and prior to reaching the lysosomes, cargo-containing endocytic vesicles pass through endosomal intermediates, notably, the early and late endosomes which are distinct from each other based on their content, morphology, $\mathrm{pH}$ and molecular make-up. The biosynthetic pathway targets newly synthesized lysosomal proteins through vesicles from the trans-Golgi network which eventually merge with the late endosome in a direct fashion or indirectly through delivery to the plasma membrane and subsequent endocytosis (Figure 3). The most well characterized direct pathway involves mannose 6-phosphate receptor-mediated transport of lysosomal hydrolases (Kornfeld and Mellman, 1989). Lysosomes are by no means just the terminal point of the endocytic pathway. Several trafficking routes deliver cargo to the 
lysosome. In addition, some specialized lysosomes have the machinery to fuse with the plasma membrane and are thus capable of secreting undigested contents. To mediate some of these processes, lysosomes must traffic to merge with the cargo-containing vesicles or with the plasma membrane. These trafficking processes are made possible by microtubule-associated mobility of lysosomes. For instance, Pu et al. (2015) recently described a multi-subunit complex BLOC-1-related complex (BORC), which promotes movement of lysosomes to the cell periphery by recruiting the GTPase Arl8 to the lysosomal membrane. Arl8 then, in turn, initiates the coupling of lysosomes to the microtubule plus-end-directed kinesin motors. Centripetal and centrifugal lysosomal movements have also been described. Their respective GTPases and effectors were shown to be Rab7 and Rab-interacting lysosomal protein (RILP), and Arl8 and SKIP. These effectors respectively recruit minus-end-directed microtubule motor DyneinDynactin to lysosomes for centripetal movement or plus-end-directed microtubule kinesin motors for centrifugal movement (Cantalupo et al., 2001; Rosa-Ferreira and Munro, 2011). TRPLM1 activity, which results in $\mathrm{Ca}^{2+}$ release from lysosomes, was recently reported to regulate lysosomal motility, positioning and tubulation while the cholesterol sensor ORPIL controls the association of late endosomes with motor proteins and their positioning in cells ( $\mathrm{Li}$ et al., 2016; Rocha et al., 2009). Undoubtedly, these processes are more complex than previously envisaged.

Beyond motility, subsequent fusion with cargo-containing vesicles and degradation of cargo, lysosomes are restored through lysosomal reformation mechanisms. Reactivation of mTORC1 signaling following prolonged starvation was shown to trigger tubulation from autolysosomes (fused autophagosomes and lysosomes). Vesicles which matured into functional lysosomes budded from such tubules (Chen and Yu, 2017; Yu et al., 2010). Furthermore, a mutation in Spinster, a putative lysosomal permease, and sugar transporter, which ablated mTORC1 reactivation following protracted starvation, resulted in enlarged autolysosomes, which could not bud proto-lysosomal tubules (Rong et al., 2011). To complete lysosomal reformation following autophagy, various factors have been reported to mediate scission of lysosomes from proto-lysosomal tubules of autolysosomes including PIP5K1A and the GTPase, dynamin 2 (Rong et al., 2012; Schulze et al., 2013). The relevance of lysosomal reformation is further emphasized by the motor neuron disease, spastic paraplegia, whose gene products, Spastizin and Spatacsin are unable to mediate lysosomal reformation (Chang et al., 2014). 


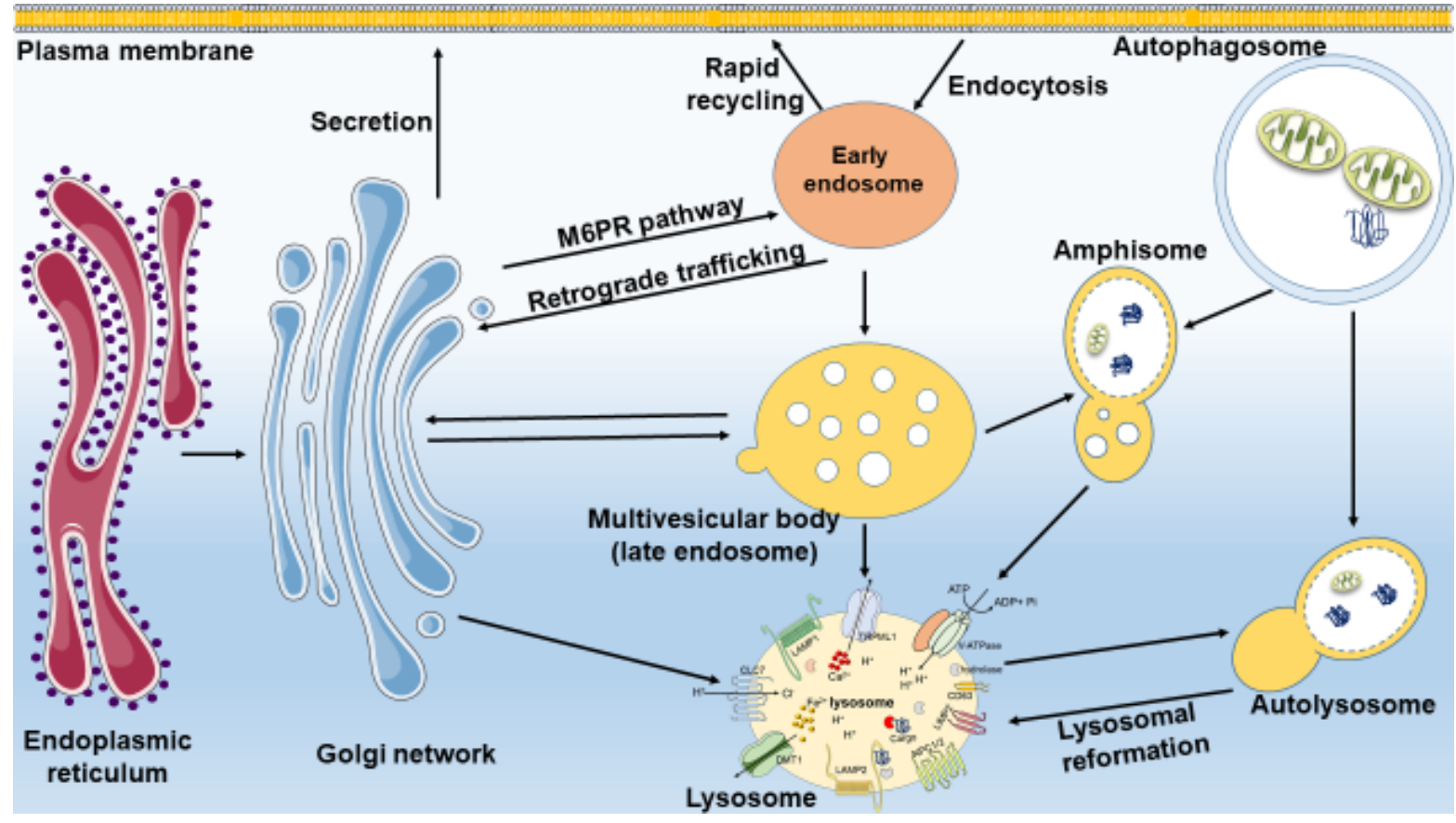

Figure 3| Pathways of lysosomal biogenesis - The biosynthetic pathway interacts with the endocytic (and autophagy) pathways during lysosomal biogenesis. Newly synthesized lysosomal proteins in the ER are processed and transported via the Golgi network directly to endocytic pathway or indirectly through secretion and subsequent endocytosis from the plasma membrane. Through the endocytic pathway, cargo reaches the lysosome via maturation and fusion events. Cargo that is sequestered by the autophagosome is delivered via autophagy to the lysosome, with a subsequent budding of new lysosomes from the autolysosome. (original illustration, KF Yambire)

Lysosomal function ultimately depends on the coordinated action of several mechanisms including their trafficking towards cargo, integration of biosynthetic pathways of lysosomal proteins and endocytosis. It was thus rational to postulate the presence of a concerted program of regulation of lysosomal biogenesis and function. This led to the discovery, by Andrea Ballabio and colleagues, in 2009, of a transcriptional program, which coordinated the expression of lysosomal genes including those of lysosomal hydrolases and lysosomalassociated membrane proteins (Sardiello et al., 2009). Employing a bioinformatic approach, these authors identified that most lysosomal genes shared a palindromic 10-base pair GTCACGTGAC motif in their promoters. They would later term this motif the coordinated lysosomal expression and regulation (CLEAR) element and find that its sequence overlaps that 
of the E-box which is targeted by basic helix-loop-helix (bHLH) transcription factors (Sardiello et al., 2009).

In addition, they described members of the microphthalmia-transcription factor E (MiT/TFE) subfamily to bind to the CLEAR element and demonstrated that overexpression of TFEB, a member of this subfamily in humans, was sufficient to drive expression of lysosomal genes and significantly increase the number of lysosomes per cell (Sardiello et al., 2009). Subsequently, it was demonstrated that, in addition to regulating lysosomal biogenesis, TFEB mediated the expression of autophagy genes, thereby coupling these two fundamental processes of lysosomal function (Palmieri et al., 2011; Settembre et al., 2011). It is noteworthy that following the discovery of TFEB and its role in regulating lysosomal biogenesis and function, several investigations focused on the other members of MiT/TFE subfamily. These studies demonstrated that MITF and TFE3 in particular mediated lysosomal biogenesis and function similar to TFEB in a context or cell type-specific manner, thus illustrating the redundancy between these transcription factors (Martina et al., 2014; Nezich et al., 2015; Ploper and De Robertis, 2015).

Conversely, the postulation of a coordinated program of regulation of lysosomal biogenesis and function is certainly not restricted to transcriptional activation. Indeed, a zinc finger family DNA-binding protein with a SCAN and a KRAB domain, ZKSCAN3 was shown to regulate autophagy and lysosomal biogenesis (Chauhan et al., 2013). ZKSCAN bound to promoter regions of several lysosomal and autophagy-related genes; however, unlike TFEB, ZKSCAN repressed expression of lysosomal and autophagy genes. Consistently, silencing ZKSCAN resulted in an increase in the number of lysosomes as well as the induction of autophagy (Chauhan et al., 2013). More recently, the bromodomain-containing protein 4 (BRD4) was also found to negatively regulate lysosomal and autophagy functions through RNAi screening and transcriptome analyses. BRD4 was shown to bind to acetylated histones in the promoter regions of lysosomal and autophagy genes and suppress their expression. Consistently, it was demonstrated that nutrient deprivation via AMPK activated the histone deacetylase SIRT1 through its dissociation from the inhibitory molecule DBC1. Histone deacetylation by SIRT1 prompted BRD4 dissociation from the promoters of lysosomal and autophagy genes thereby inducing their expression (Sakamaki et al., 2017). Despite this progress, discovering more transcriptional networks of lysosomal-autophagic function regulation is still the subject of several ongoing investigations. 
Following the striking discovery of the role of MiT/TFE factors in lysosomal-autophagic function, the lysosomal and signaling fields were interested in elucidating the mechanisms that controlled the activity of these transcription factors. In the initial report of TFEB as a regulator of lysosomal

\section{$A_{\text {Nutrient repletion }}$}
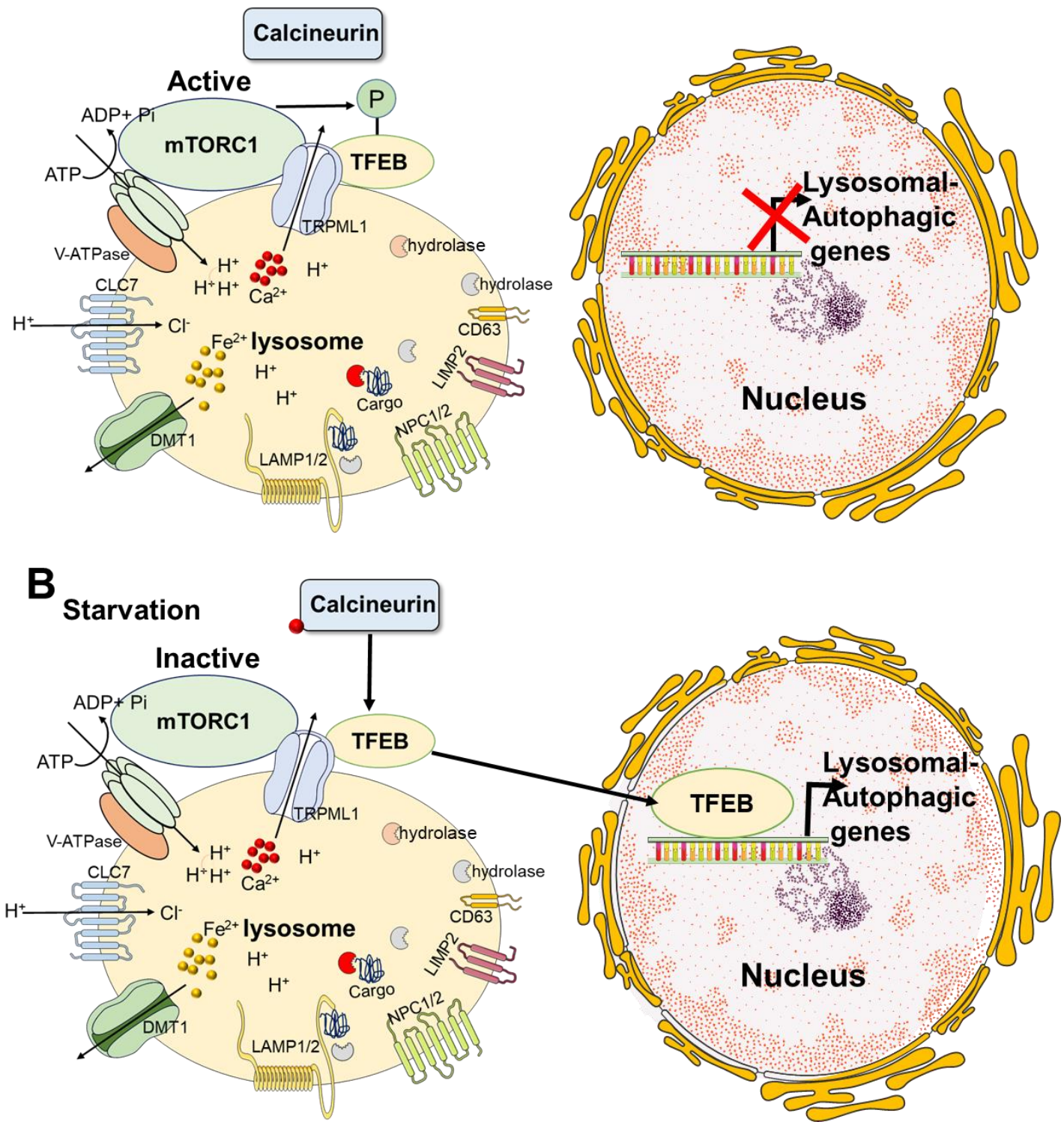

Figure 4| Transcriptional regulation of lysosomal biogenesis - A TFEB is phosphorylated by mTORC1 under nutrient-replete condition thereby blocking its nuclear translocation and activity. B Under starvation conditions, mTORC1 inactivity and calcineurinmediated dephosphorylation of TFEB allow its nuclear translocation and transcriptional activation of lysosomal and autophagy genes. (original illustration, KF Yambire) 
biogenesis, amino acid starvation was found to promote nuclear translocation of TFEB, a required step for its transcriptional function (Sardiello et al., 2009). Nuclear-cytoplasmic relocation of TFEB was found subsequently to be under the control of mTORC1 (Martina et al., 2012; Roczniak-Ferguson et al., 2012; Settembre et al., 2012). These studies found that nutrient withdrawal, which led to $\mathrm{mTORC} 1$ inhibition, as well as $\mathrm{mTORC} 1$ catalytic inhibitors, led to the rapid relocation of TFEB into the nucleus (Figure 4B). On the contrary, under nutrientreplete conditions, TFEB was phosphorylated by mTORC1 on Serine 142 and Serine 211, leading to its binding to 14-3-3 proteins and cytoplasmic retention (Figure 4A). This mechanism of regulation was found to be shared between members of the MiT/TFE family, especially MITF and TFE3 ((Martina et al., 2012; Roczniak-Ferguson et al., 2012). In addition, MEK/ERK and AKT have also been shown to regulate the nuclear-cytoplasmic translocation of MiT/TFE family members (Palmieri et al., 2017; Settembre et al., 2011). While the activity of these kinases negatively regulates TFEB by phosphorylation leading to its cytoplasmic retention, lysosomal calcium signaling has been shown to positively mediate TFEB activity through its dephosphorylation and subsequent nuclear translocation (Medina et al., 2015). Like the endoplasmic reticulum, the lysosomal lumen is a storage site for $\mathrm{Ca}^{2+}$. Following nutrient withdrawal, activation of the lysosomal TRPML1 channel releases $\mathrm{Ca}^{2+}$ into the cytosol, which activates calcineurin, a serine-threonine phosphatase. Calcineurin dephosphorylates TFEB prompting its rapid nuclear relocation. Consistent with this mechanism, calcium chelation or TRPML1 silencing blocked nuclear translocation of TFEB under starvation conditions (Medina et al., 2015).

\subsubsection{Lysosomal function}

\subsubsection{Lysosomes as major cellular catabolic centers}

Although lysosomes have evolved to play other pivotal roles in metabolism such as nutrient sensing, serving as reservoirs of amino acids and ions, and transcriptional regulation, they remain the principal sites of intracellular digestion of macromolecules. Cargo destined for degradation is delivered to the lysosome through endocytic (including pinocytic and phagocytic) and autophagic pathways. These pathways terminate in the lysosome where its hydrolytic enzymes mediate the degradation of diverse macromolecules.

The endocytic pathway targets extracellular cargo to the lysosome through budding of vesicles from the plasma membrane. The endocytic pathway is also reached by biosynthetic cargo from the Golgi network directly or indirectly following secretion and subsequent retrieval from the 
plasma membrane (Figure 3). The endocytic pathway begins with the budding of coated vesicles of about 60 to $100 \mathrm{~nm}$ in diameter from the plasma membrane. The vesicles undergo multiple rounds of interaction and fusion events with early endosomes near the plasma membrane resulting in the gradual remodeling and maturation of these early endosomes (Klumperman and Raposo, 2014). These fusion and maturation events are pivotal for the acquisition of functional and biological markers. One of such markers is Rab5, which has been shown to be necessary for the biogenesis of the endolysosomal system (Zeigerer et al., 2012). In this study, liver-specific silencing of Rab5 was reported to lead to a striking decrease in the number of early endosomes and, as a consequence, a decrease in the number of late endosomes, further illustrating the dynamic interplay between these endosomal subtypes (Zeigerer et al., 2012).

Not all contents of early endosomes are destined for degradation given the variety of cargo that they receive. As such, functionally, endosomes sort cargo for degradation or recycling. Endosomes extend tubules which contain materials to be recycled while cargo targeted to the lysosomes remain in the sorting endosomal vacuole. Another characteristic distinction of the vacuoles is the presence of intraluminal vesicles which are absent in tubules (Saftig and Klumperman, 2009). These sorting endosomal vacuoles fuse with and mature into late endosomes while exchanging biological markers like Rab5 for Rab7 (Rink et al., 2005). Multiple fusion events result in an increase in the number of intraluminal vesicles in the late endosomes, thus giving them the characteristic appearance which is commonly referred to as multivesicular bodies. Late endosomes, when formed, are typically competent to fuse with the lysosome where the contents are either degraded or resident lysosomal proteins are retained (Klumperman and Raposo, 2014).

Autophagy is a conserved and highly regulated pathway that targets intracellular cargo and even organelles to the lysosome for degradation. Although the concept existed for a long time, since the lysosomes were considered to be the 'garbage bags' of the cell, the mechanisms governing the autophagic process were not appreciated. This was probably due to the unavailability of technologies to study such dynamic processes in the cell. Genetic studies in yeast, in the early 1990s, led to the discovery of a series of autophagy-related (ATG) genes (Klionsky et al., 2003). Since then, the autophagy field has expanded exponentially. Most autophagy events are categorized into either one of three classes: macroautophagy, microautophagy, or chaperone-mediated autophagy (CMA) (Figures 5 and 6). Macroautophagy is the most broadly studied class of autophagy since it is thought to be the most predominant type. 


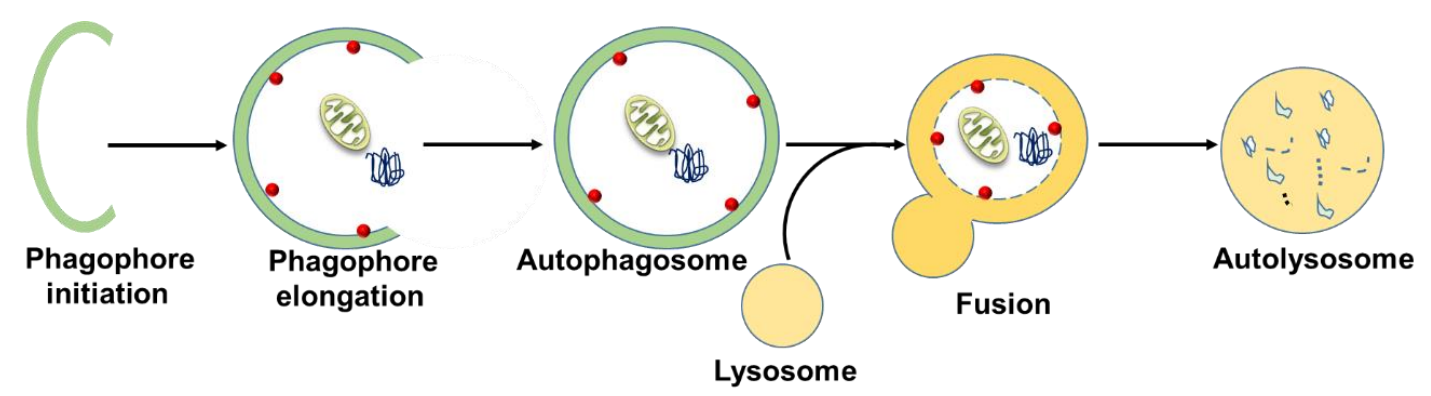

Figure 5| Macroautophagy pathway degrades cellular contents within lysosomes

- During Macroautophagy, cytoplasmic portions or whole organelles are sequestered by an isolation membrane, which elongates and eventually encloses the cargo in an autophagosome. The autophagosome delivers its content via fusion with lysosome to form dynamic structures called autolysosomes, whose contents are degraded by lysosomal hydrolases. (original illustration, KF Yambire)

During macroautophagy, an isolation membrane (phagophore), whose origin is still the subject of considerable debate and investigation, sequesters a portion of the cytoplasm into a double membrane-bound dynamic organelle termed the autophagosome (Mizushima and Komatsu, 2011). After maturation of the autophagosome, it then fuses with the lysosome to become the autolysosome, whose contents are degraded by the lysosomal hydrolases (Figure 5). Autophagosomes may form amphisomes by fusing with late endosomes before finally fusing with the lysosome (Shen and Mizushima, 2014).

Microautophagy and CMA, by contrast, target intracellular material to the lysosome in an autophagosome-independent manner (Figure 6). For instance, in microautophagy, the lysosome engulfs small cytoplasmic material via inward invagination of its membrane (Figure 6A). In CMA, as illustrated in Figure 6B, proteins containing a pentapeptide motif in their amino acid sequence, KFERQ, are translocated across the lysosomal membrane via a multimeric translocation complex with LAMP2A as receptor and HSC70 as a chaperone (Kaushik and Cuervo, 2012). 
A

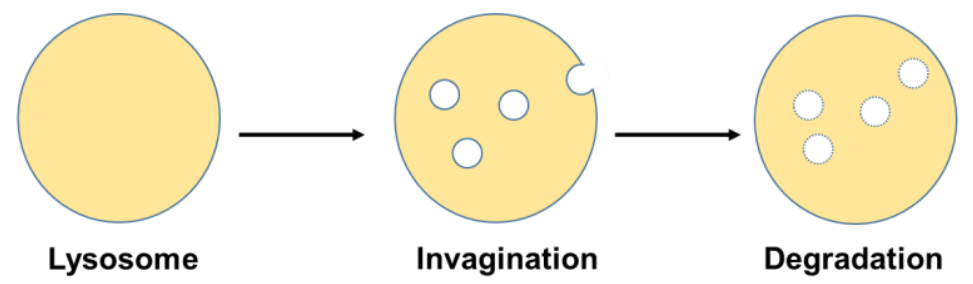

B

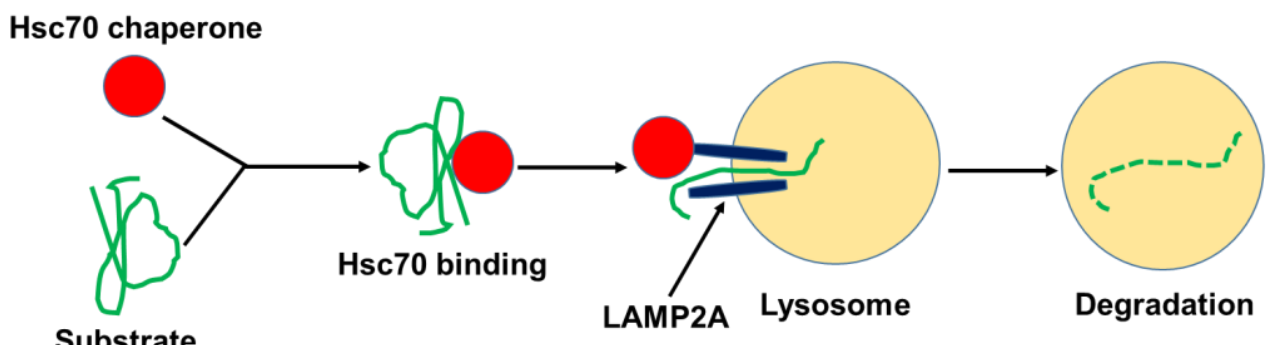

Figure 6| Microautophagy and chaperone-mediated autophagy pathways mediate direct lysosomal engulfment of cytoplasmic cargo - A During microautophagy, lysosomal membrane invagination engulfs small cytoplasmic material, which is then degraded by the lysosomal hydrolases. B In chaperone-mediated autophagy, pentapeptide motifs in the amino acid sequence of substrate proteins are recognized by Hsc70 chaperone, which targets these proteins to the lysosome via a multimeric translocation complex with LAMP2A as the receptor. (original illustration, KF Yambire)

\subsubsection{The lysosome as a signaling hub}

Lysosomes have emerged as key regulators of metabolism due to their physical and functional interaction with mTORC1, the master growth regulator (Laplante and Sabatini, 2012; Perera and Zoncu, 2016). The evolutionary success of this role stems from the primitive function of lysosomes as catabolic centers of the cell and hence reservoirs of nutrients. It would seem logical to couple the master growth regulator to the source of nutrients which drive growth (Chantranupong et al., 2015). Intriguingly, mTORC1 modulates mass accumulation via control of anabolic and catabolic processes by integrating cues from lysosomes such as nutrients and growth factors (Laplante and Sabatini, 2012; Ma and Blenis, 2009). This is the same signaling route, which is targeted during nutrient scarcity, where $\mathrm{mTORC} 1$ is inactivated leading to TFEB nuclear translocation and transcriptional activation of catabolic processes to restore nutrient levels (Martina et al., 2012; Roczniak-Ferguson et al., 2012; Settembre et al., 2012). Notably, the mechanism of mTORC1 recruitment to the lysosomal surface by amino acids is well 
characterized (for a recent review see Perera and Zoncu, 2016). The lysosomal lumen is also a reservoir for $\mathrm{Ca}^{2+}$, whose signaling role as a ubiquitous second messenger is widely appreciated. Lysosomal $\mathrm{Ca}^{2+}$ concentration is about one thousand-fold higher than cytosolic $\mathrm{Ca}^{2+}$ levels (Christensen et al., 2002). The role of lysosomal calcium in calcineurin-dependent activation of TFEB was only recently demonstrated (Medina et al., 2015). However, it remains to be elucidated which other cellular processes require lysosomal calcium release.

\subsubsection{Lysosomes and the immune response pathways}

There is now ample evidence in support of the essential role of lysosomes in immune response pathways. Indeed, the function of various immune cell types like macrophages and microglia stems from the unique lysosomes they possess to degrade and inactivate pathogens. In addition, lysosomes are equipped with the machinery to drive the innate immune response (Kawai and Akira, 2010). Although the plasma membrane is the cell's first point of contact with invading pathogens, these pathogens ultimately reach the lysosome via the endocytic pathway. Various pattern recognition receptors including TLR7 and TLR9 have been known to localize to the endolysosomal system following stimulation. TLR9 for example has been identified as the receptor for the unmethylated CpG-rich DNA motifs, which are present in bacteria and mitochondrial DNA (O'Neill et al., 2013; West and Shadel, 2017). Upon sensing these motifs in the late endosome or lysosome, TLR9 recruits its signaling molecule, myeloid differentiation primary-response protein 88 (MYD88). MYD88 signaling drives the transcriptional activity of interferon-regulatory factor 7 (IRF7) leading to the induction of type I interferon genes, which initiate the immune response as well as the production of cytokines (Barbalat et al., 2011). In addition, the lysosome plays a pivotal role in adaptive immunity through antigen presentation. The lysosomal and endoplasmic reticulum systems have been shown to degrade, process and load antigen peptides onto major histocompatibility complex (MHC) molecules which are then trafficked to the cell surface for presentation (Gao et al., 2017; Hsing and Rudensky, 2005).

Although the exact relationships between altered lysosomal malunction and immune response induction are currently under intense investigation, there is already a substantial body of data that links LSDs and irregularities in the immune system (reviewed in Kawai and Akira, 2010; Platt et al., 2016; Rigante et al., 2017). 


\subsubsection{Lysosomes: regulators of iron homeostasis}

Iron is an essential trace element that is required as a functional component of many proteins, including the formation of heme and iron-sulfur clusters. As a cofactor in several metabolic processes, iron participates in oxidative phosphorylation as well as in the regulation of the activity of ribonucleotide reductases, which are central to nucleotide synthesis (Bourdon et al., 2007; Guittet et al., 2001; Rouault, 2013). In addition, iron was recently found to play a crucial role in mitochondrial biogenesis. It was demonstrated in mouse myotubes that iron chelation results in a marked decrease in mitochondrial transcripts and proteins, which could be reversed by iron repletion (Rensvold et al., 2013).

Given the essential role of iron as illustrated before, its bioavailability is pivotal even for cell survival. To this end, iron homeostasis is maintained via a tightly regulated process. The endolysosomal system is involved in the two main pathways that make iron available to the cell for metabolic and other processes. For instance, ferric iron $\left(\mathrm{Fe}^{3+}\right)$ from the bloodstream is endocytosed when it binds to transferrin which in turn binds to transferrin receptors at the cell surface. The transferrin receptor-transferrin- $\mathrm{Fe}^{3+}$ complex is endocytosed via a clathrinmediated process. The endocytic vesicles ultimately reach the late endosome/lysosome where the activity of the metalloreductase STEAP3 reduces $\mathrm{Fe}^{3+}$ to ferrous iron $\left(\mathrm{Fe}^{2+}\right) . \mathrm{Fe}^{2+}$, released from transferrin, is then transported by the divalent metal transporter 1 (DMT1) into the cytosol (Lane et al., 2015; Rouault, 2013) as illustrated in Figure 7

Owing to the reactive and damaging nature of free iron via the Fenton reaction, excess amounts are sequestered by the cytosolic 24-subunit heteropolymer protein, ferritin (Kurz et al., 2008). Access to iron sequestered by ferritin is mediated by ferritinophagy where ferritin is targeted to the autophagosome via its receptor NCOA4 (Biasiotto et al., 2016; Mancias et al., 2014). Following autolysosome formation, ferritin is degraded and the iron is released, reduced and transported to the cytosol via DMT1 (Figure 7) 


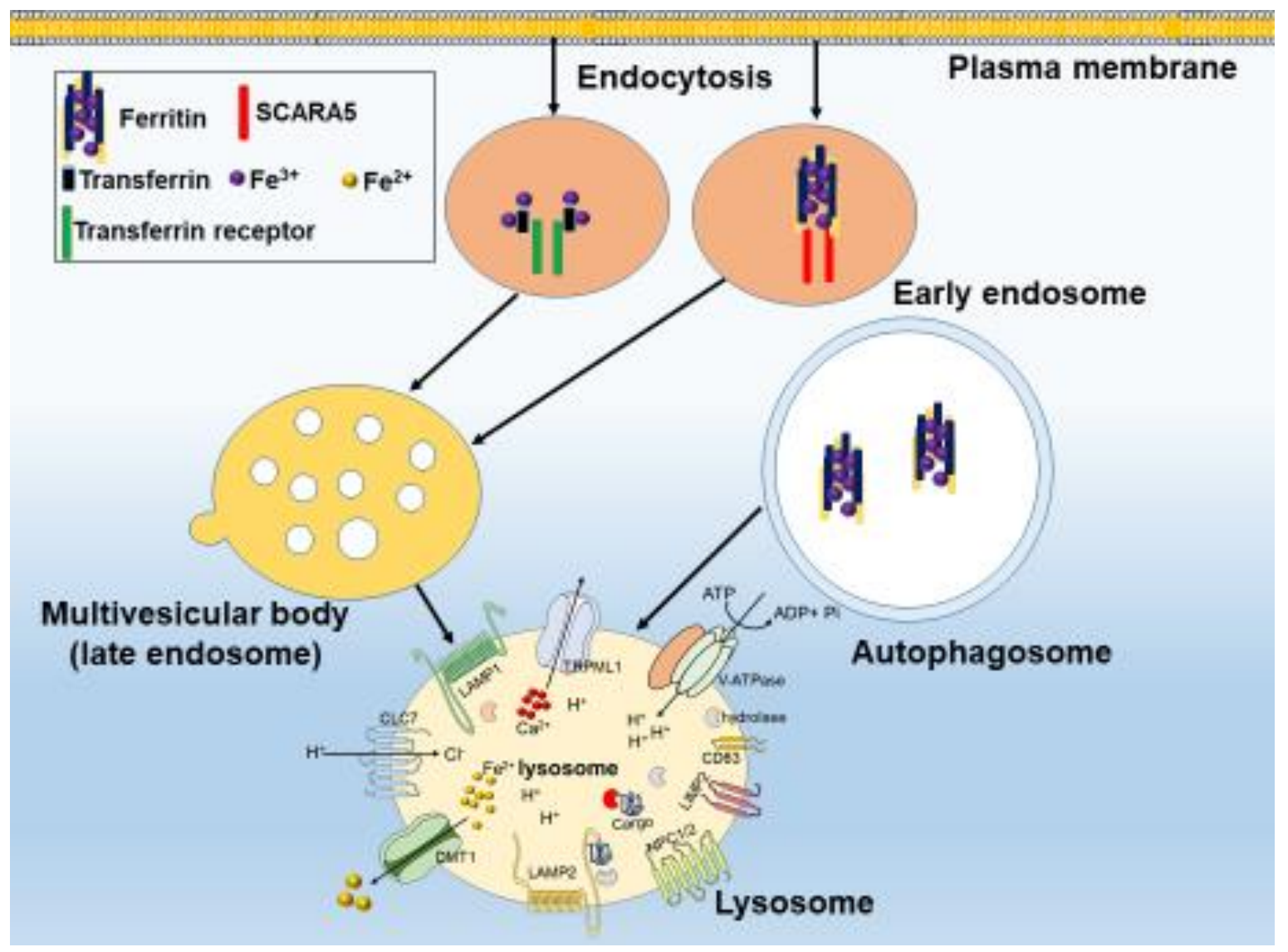

Figure 7| Maintenance of iron homeostasis - Lysosomes are central to the process of maintaining cellular iron homeostasis. Transferrin-dependent and -independent pathways deliver ferrous iron via endocytosis to the lysosome, where it is reduced and exported as free iron into the cytoplasm. In addition, cytoplasmic iron, which is sequestered by ferritin, is delivered via autophagy to the lysosome. Lysosomal hydrolases degrade ferritin and make iron available to the cell. (original illustration, KF Yambire)

Intriguingly, the transferrin-independent and cell-type specific pathway of iron delivery via the Scavenger Receptor Class A Member 5 (SCARA5) protein also requires the endolysosomal system. SCARA5 acts as a ferritin receptor mediating endocytosis of iron-bound ferritin directly from the bloodstream. The SCARA5-Ferritin complex is delivered to the lysosome where it is degraded and iron is once again released, reduced and transported into the cytosol (Li et al., 2009). This pathway is also depicted in Figure 7.

Maintenance of iron homeostasis depends on the activity of several proteins, whose posttranscriptional regulation by iron regulatory protein (IRP)1 and IRP2 provides another 
mechanism of cellular iron regulation. IRP1 and IRP2 sense iron levels in the cytosol and regulate the transcripts of genes encoding for key proteins required for iron homeostasis (Lane et al., 2015; Rouault, 2013). When cytosolic iron levels are low, IRPs bind iron-responsive elements (IREs) in the transcripts of these genes and interfere with translation by preventing the assembly of the translational machinery leading to decreased levels of ferritin for instance. Conversely, IRP binding to transferrin receptor 1 (TFRC) mRNA stabilizes it, leading to increased transferrin receptor synthesis. However, under conditions of iron overload, iron binds to IRPs resulting in their dissociation from IREs. Consequently, translation of ferritin is enhanced while TFRC is degraded by endonucleases (Biasiotto et al., 2016; Rouault, 2013).

\subsection{Lysosomal storage disorders}

The crucial role that lysosomes play in cellular metabolism implies that altered lysosomal function would contribute greatly to the pathogenesis of several metabolic diseases. Indeed, the inability of the lysosome to degrade cargo, resulting in their accumulation, or export nutrients underlies lysosomal storage disorders (LSDs). LSDs are inborn errors of metabolism with an approximate combined incidence of 1 in 5000 live births (Platt et al., 2012). LSDs are caused mainly by heritable mutations in genes that encode lysosomal hydrolases (e.g., GBA in Gaucher's diseases and GAA in Pompe's disease) and permeases (e.g., NPC1/2 in Niemann-Pick type C and MCOLN1 in Mucolipidosis type IV). Neurodegeneration is a hallmark of most LSDs, probably because neurons have limited regenerative capacity and may be particularly dependent on lysosomal function to maintain neuronal health (Perera and Zoncu, 2016). Increasingly, mutations leading to LSDs are emerging as risk factors for late-onset neurodegenerative disorders like Alzheimer's disease and Parkinson's disease (Osellame and Duchen, 2014). Although around 70 LSDs have been described, in the context of this study, only two kinds of LSDs will be discussed in the following sections. 


\subsubsection{Niemann-Pick disease}

Niemann-Pick diseases (NP) are a group of sphingolipid storage disorders, which are characterized by the accumulation of different lipid species in lysosomes (Platt, 2014). Three distinct forms of NP have been described: Types A, B and C. Types A and B are caused by a deficiency in the lysosomal enzyme, acid sphingomyelinase (ASM), while Type $C$ is caused by defects in two distinct lysosomal cholesterol efflux proteins, NPC1 and NPC2. Though the etiologies may differ, these diseases display common biochemical and clinical features (Schuchman and Desnick, 2017).

In NP types A and B (NPA and NPB), ASM deficiency, caused by mutations in SMPD1, leads to the accumulation of sphingomyelin (ASM substrate) in the lysosome. The hallmark of NPA is hepatosplenomegaly and progressive neurodegeneration, with most patients unable to thrive beyond 3 years of age mostly due to respiratory failure (Schuchman and Desnick, 2017). NPB is phenotypically variable; although patients also display hepatosplenomegaly, they typically do not show central nervous system (CNS) pathologies. Defects in NPA and NPB are modeled by the $\mathrm{ASM}^{-1-}$ mice with liver fibrosis and brain deficits consistent with clinical findings in patients (Schuchman and Desnick, 2017).

In NPC, deficiency of the lysosomal cholesterol efflux proteins NPC1 and NPC2 result in impaired lipid trafficking and accumulation of unesterified cholesterol in the liver, spleen, and CNS. As a result, NPC is characterized by progressive neurological deficits (Patterson et al., 2012). NPC1 is a lysosomal transmembrane protein while NPC2 resides in the lysosomal lumen. NPC1 and NPC2 are thought to cooperate in cholesterol efflux from the lysosome, where NPC2 binds cholesterol and transfers it to NPC1, which shuttles it through the glycocalyx to the lysosomal membrane where it is released (Davies and loannou, 2000; Friedland et al., 2003). The NPC1 ${ }^{-1}$ mice closely model defects in NPC with progressive neural deficits and ataxia. In addition, impaired lysosomal calcium homeostasis in NPC has been shown to result in reduced calcium release, which blocks late endolysosomal fusion events. This results in glycosphingolipid deposition in late endosomes and lysosomes (Lloyd-Evans et al., 2008). 


\subsubsection{Glycogen storage disease type II (Pompe's disease)}

The concept of LSDs was established based on studies of Pompe's disease, which incidentally was the first LSD to be described. Pompe's disease is caused by mutations in acid alphaglucosidase (GAA) leading to a deficiency of its gene product, the lysosomal glycogenmetabolizing enzyme. Pompe's disease is an inherited metabolic myopathy, which is characterized by striated muscle accumulation of intralysosomal glycogen (Raben et al., 2012). Cardiac hypertrophy, progressive muscle weakness, and respiratory dysfunction are hallmarks of Pompe's disease, and patients with severe forms of infantile Pompe's disease fail to thrive beyond 1 year of age (DeRuisseau et al., 2009; van der Ploeg and Reuser, 2008). The GAA ${ }^{-1-}$ mouse model mimics the classical infantile form of human Pompe's disease. These animals develop generalized intralysosomal glycogen accumulation with progressive skeletal and cardiac myopathy as disease manifestations (Raben et al., 2002). Although earlier studies in patient and mouse models ruled out nervous system dysfunction in Pompe's disease, recent investigations have demonstrated central and peripheral nervous system pathology in GAA ${ }^{-1-}$ mice (DeRuisseau et al., 2009; Falk et al., 2015; Turner et al., 2016). 


\subsection{Mitochondrial biology}

Aerobic respiration takes place in a membrane-enclosed sub-compartment of the eukaryotic cell. This membrane-enclosed organelle, which uses oxygen to generate energy, is now known as the mitochondrion. The word 'mitochondrion' crudely translates as thread-like granule owing to its initial description as 'granules' in insect muscles by Rudolf Albert von Koelliker around the mid-1800s. The subsequent discovery of DNA in mitochondria strongly emphasized their close relationship with bacteria, which are thought to have been phagocytosed by a protoeukaryote more than 2 billion years ago (Lane and Martin, 2010; Müller et al., 2012).

Although mitochondria have evolved to accommodate several roles in cellular metabolism, they are still referred to, in text today, as 'energy factories' of the cell. This designation originates from their crucial role in the generation of ATP, 'the energy currency of the cell' (Scheffler, 2007). Beyond ATP synthesis, mitochondria mediate a wide range of cellular functions including amino acid and fatty acid metabolism, iron-sulfur cluster biogenesis, heme and steroid synthesis, and calcium homeostasis (Nunnari and Suomalainen, 2012). In addition, mitochondria play diverse roles in the regulation of cellular processes such as autophagy and apoptosis, and cell proliferation and differentiation (Nunnari and Suomalainen, 2012; Raimundo, 2014). The important role of mitochondria in cellular physiology is further highlighted by pathologies that arise following mitochondrial malfunction (DiMauro, 2004). Mitochondrial dysfunction, with etiologies stemming from defects in genes from both genomes encoding mitochondrial proteins, has been shown to result in several diseases including myopathies and neurodegenerative disorders (DiMauro, 2004).

\subsubsection{Structure and morphology of mitochondria}

Mitochondria were usually depicted as sausage-like-shaped, static organelles in the cell with a diameter of $0.5-1.0 \mu \mathrm{M}$, a notion which has since been challenged by recent evidence that mitochondria constantly undergo fusion and fission in response to cellular cues (Pernas and Scorrano, 2016). Two specialized membranes with different functions enclose mitochondria, making them distinct from all other organelles within the cell (Figure 8). 


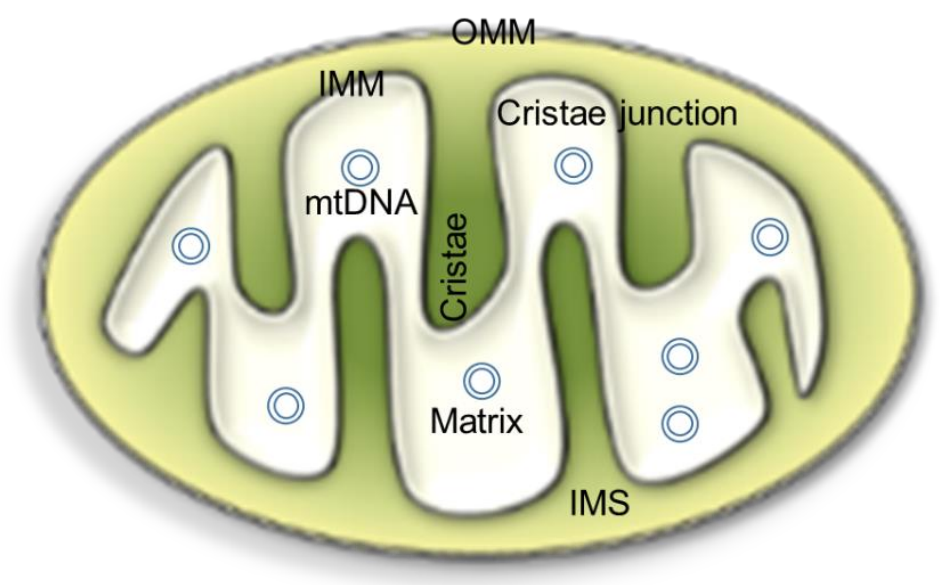

Figure 8| Ultrastructure of the mitochondrion - The outer mitochondrial membrane (OMM) surrounds the intermembrane space (IMS) and inner mitochondrial membrane (IMM). The IMM, which is folded into the cristae, encloses the mtDNA-containing matrix. The cristae house the respiratory chain complexes and ATP synthase. (original illustration, KF Yambire)

The outer mitochondrial membrane (OMM) serves as the barrier between the cytoplasm and the intermembrane space (IMS), preventing small molecule diffusion while protecting the cell from deleterious mitochondrial products like death cues (pro-apoptotic factors) or even the mitochondrial genome (mtDNA). However, the OMM contains the $\beta$-barrel protein, porin, also known as voltage-dependent anion channel (VDAC), which confers membrane permeability to ions, small metabolites and non-electrolytes of less than 8kDa (Colombini, 1980; Zalman et al., 1980). In addition, mitochondrial motility along microtubules and cytoskeletal filaments is due to interaction with proteins of the OMM. Mitochondria also engage other sub-cellular compartments like the nucleus and endoplasmic reticulum through the OMM (Pernas and Scorrano, 2016).

The cardiolipin-rich inner mitochondrial membrane (IMM), which creates the two subcompartments, IMS and matrix (Figure 8), is further divided into two subdomains. One of the domains, the inner boundary membrane (IBM) forms contacts with the OMM at sites where the protein import machinery is localized (Reichert and Neupert, 2002).

The other domain of the IMM is highly convoluted forming a series of invaginations known as the cristae (Figure 8). The IBM and cristae membrane join at narrow necks referred to as cristae junctions. The cristae house the assembled respiratory chain complexes and supercomplexes as well as the proteins involved in iron-sulfur cluster biogenesis and mtDNA maintenance and 
transcription (Krauss, 2001; Pernas and Scorrano, 2016). Due to the high number of protein machinery for several processes localized to the IMM, it has an unusually high protein to lipid ratio of about 80:20, compared to that of the OMM which is 50:50. Another key feature of the IMM is optic atrophy 1(OPA1)-mediated remodeling of the cristae. Indeed, the number and morphology of the cristae may reflect a response of mitochondria to energy demands of the cell. For example, under high energy requirements, or in cells with greater respiratory rates like myocytes and neurons, the cristae are usually presented as highly folded and lamellary with a large surface area (Patten et al., 2014; Pernas and Scorrano, 2016; Rossignol et al., 2004).

The two aqueous compartments, IMS (more specifically the intra-cristae space) and matrix, respectively delimited by the OMM and IMM, are important for oxidative phosphorylation. The IMS usually has a low $\mathrm{pH}$ due to the protons that are pumped from the matrix into the IMS. The net effect is the maintenance of chemical and electrical gradients which are necessary for ATP synthesis. Some enzymes localized to the IMS are involved in heme synthesis or creatine phosphorylation. The most abundant IMS protein, cytochrome c, represents the electron shuttle connecting complexes III and IV of the respiratory chain (Herrmann and Riemer, 2010). The metabolic center of the mitochondrion is the matrix, which houses enzymes of the citric acid cycle, oxidative phosphorylation, urea cycle and beta-oxidation of fatty acids. It contains the mtDNA and the protein machinery necessary for its replication and expression (Scalettar et al., 1991).

Contrary to the depiction of mitochondria as static, rod-shaped organelles, evidence challenging this notion existed more than a century ago, even before they were referred to as 'power plants' of the cell. Indeed, in the words of Lewis and Lewis (1914), “...there are to be found in single stained preparations all gradations of size and shape from the small and large granules to short rods and long rods, to threads of varying length, to anastomosing threads and networks, which extend throughout the cytoplasm and to rings and loops of various shapes". These observations of mitochondrial fusion and fission were made from chick embryo cells in culture (Lewis and Lewis, 1914). It is intriguing that with the tools available to biologists in the early $20^{\text {th }}$ century, a report that accurately describes the modern day micrograph of mitochondria depicted in Figure 9 could be made. Nonetheless, it would take close to a century to understand the molecular basis of mitochondrial dynamics and its role in mitochondrial function. 


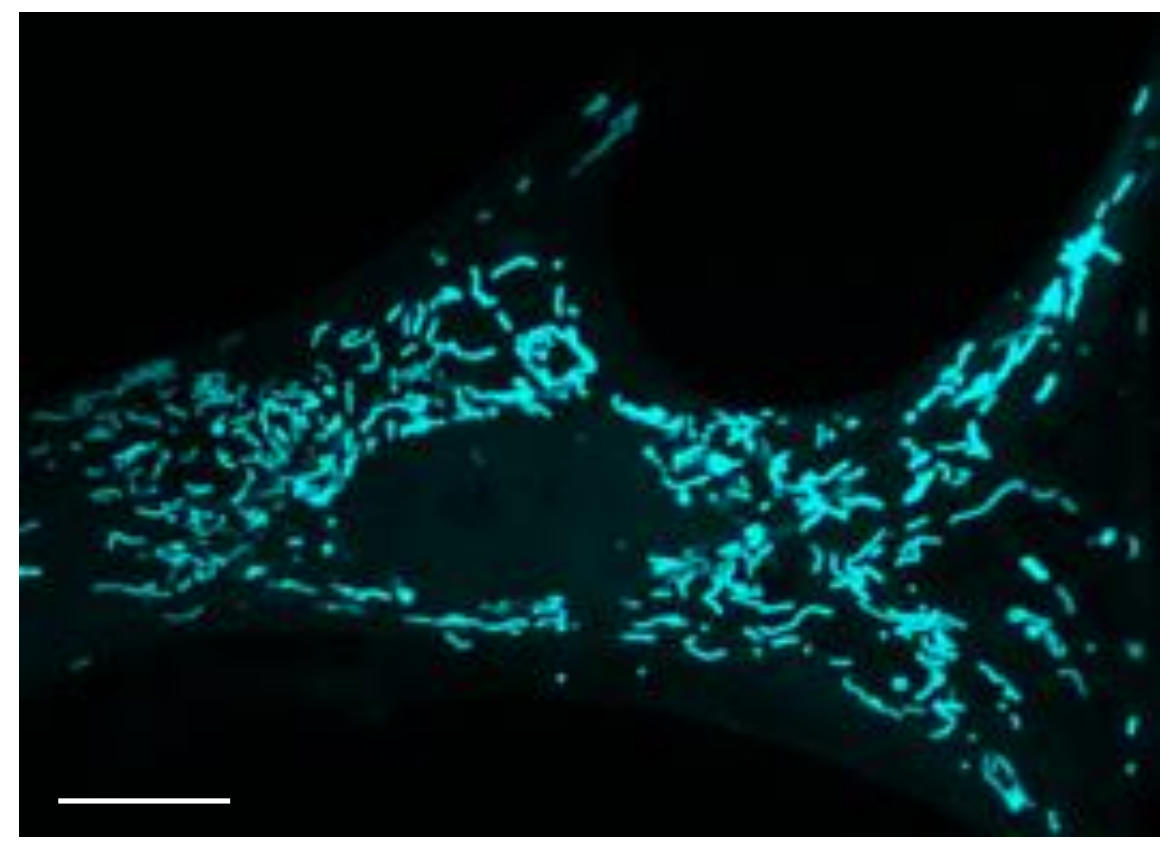

Figure 9| Mitochondrial network in mouse embryonic fibroblasts - A micrograph of MEFs transfected with a mitochondrial-targeted cyan fluorescent protein illustrates the mitochondrial network with diverse shapes and lengths of mitochondria. Scale bar : $5 \mu \mathrm{M}$.

Generally mitchondrial morphology and number vary depending on cell type and contextspecific demands, and the balance between mitochondrial fusion or fission regulates mitochondrial morphology, distribution and function (Chan, 2012). Mitochondrial dynamics plays a crucial role in mitochondrial quality control and its altered shape affects several processes including respiration, apoptosis, mtDNA stability or cellular stress responses (Chan, 2012; Pernas and Scorrano, 2016).

Mitochondrial fission is mediated by a member of the dynamin superfamily of GTPases, the dynamin-related protein 1 (DRP1) (Smirnova et al., 2001). DRP1 is a cytosolic protein, which is recruited to mitochondria by either adaptor proteins, Fission 1 (FIS1), mitochondrial fission factor (MFF), mitochondrial dynamics proteins (MiD) of $49 \mathrm{kDa}$ (MiD49) or $51 \mathrm{kDa}$ (MiD51) (Gandre-Babbe and van der Bliek, 2008; Loson et al., 2013; Palmer et al., 2013). Following the marking of a fission site, recruited DRP1 oligomerizes into a coil-shaped structure around the marked fission site, which constricts and fissions mitochondria following GTP hydrolysis. Mitochondrial fission is involved in apoptosis as well as in mitochondrial proliferation during cell division (Chan, 2012; Pernas and Scorrano, 2016). 
Mitochondrial fusion requires two distinct fusion events each for either OMM or IMM, which are also mediated by distinct fusion machinery. Fusion of the OMM is mediated by the OMMlocalized transmembrane GTPases Mitofusins 1 and 2 (MFN1 and MFN2). OPA1, the dynaminrelated GTPase, mediates IMM fusion. During fusion, MFN1 and MFN2 tether two closely apposed mitochondria by forming homotypic or heterotypic complexes in trans between them, thereby promoting fusion (Chen et al., 2003; Koshiba, 2004). OPA1 is however not required on adjacent membranes for IMM fusion (Song et al., 2009). Mitochondrial fusion is thought to be relevant for maintaining a healthy population of mitochondria where toxic mitochondrial products or mutant mtDNA is diluted among mitochondria. (Pernas and Scorrano, 2016)

\subsubsection{Mitochondrial genome}

Except for the nucleus, mitochondria are the only organelles in animal cells to possess their own genetic material. The origin of mtDNA has been the subject of debate since its discovery in 1963 as DNAse-sensitive threads inside mitochondria (Nass and Nass, 1963), although its presence is consistent with the endosymbiotic theory of mitochondrial acquisition as a whole. It is thought that most genes of the $\alpha$-protobacterium were lost or transferred to the nucleus during evolution (Gray et al., 1999), leaving only the circular, compact and double-stranded mtDNA molecule with 16,569 nucleotides in humans for example as depicted in Figure 10. Each human mtDNA molecule (mostly $10^{3}-10^{4}$ copies/ cell in mammals) codes for 37 genes, comprising 13 polypeptides of the respiratory chain, 2 ribosomal RNAs (rRNAs) and 22 transfer RNAs (tRNAs). All mammalian mtDNA genes lack introns and there is only one non-coding region, which is also referred to as the control region because it contains the heavy strand and light strand promoters (HSP and LSP) for mtDNA transcription and replication (Anderson et al., 1981). Due to uneven guanidine content, the mtDNA strands can be separated into a heavy $(\mathrm{H})$ and a light $(\mathrm{L})$ strand using alkaline $\mathrm{CsCl}$ density gradient centrifugation (Berk and Clayton, 1974). The $\mathrm{H}$ strand encodes both rRNAs, 12 of the 13 mRNAs and 14 of the 22 tRNAs. The rest are encoded by the light strand (Figure 10). 


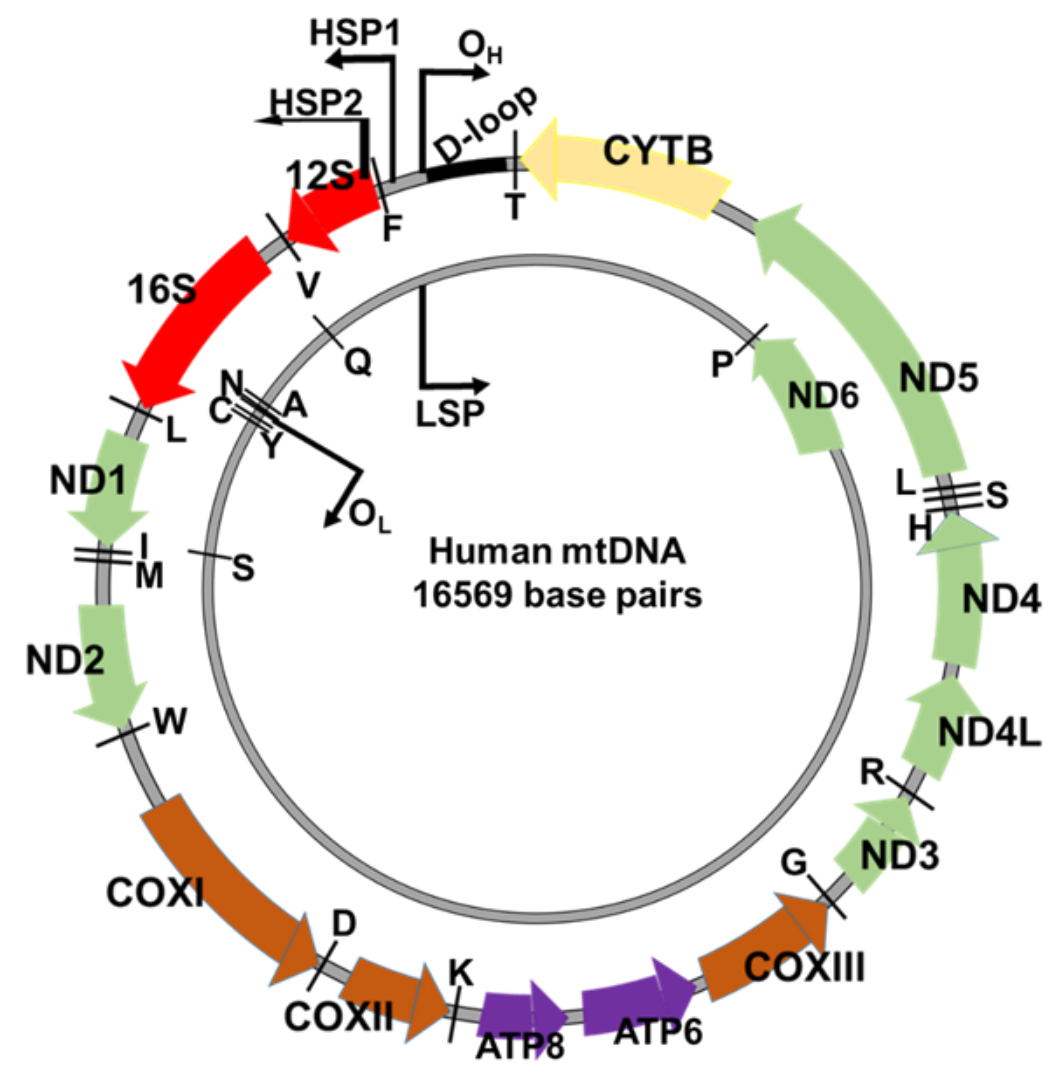

Figure 10| Human mtDNA map - Respiratory chain subunits are color-coded for each complex; ribosomal RNAs, $12 \mathrm{~S}$ and 16S have been depicted in red. Mitochondrial transfer RNAs and their corresponding single-letter amino acid codes have been indicated as black bars (original illustration, KF Yambire)

mtDNA is organized into compact ovoid structures called nucleoids, which are diverse in size with an average diameter of about 100nm (Brown et al., 2011; Kukat et al., 2011). The mitochondrial transcription factor A (TFAM), which binds to mtDNA promoters and acts a transcription factor, is also the principal core protein of the nucleoid. As a packaging factor, there are about 450 TFAM binding sites on the 16kb mammalian mtDNA where it localizes exclusively (Kaufman et al., 2007). Interestingly, when not bound to mtDNA, TFAM is unstable and is usually targeted for degradation by the LON proteases (Matsushima et al., 2010).

Since defects in mtDNA result in various human pathologies, understanding its maintenance has garnered a lot of interest over the years. Indeed, the machinery for mtDNA replication is known to consist of the DNA polymerase $\mathrm{Y}$ (PoIG), the mtDNA helicase (TWINKLE), the mitochondrial single-stranded binding protein (mtSSB), and mtDNA topoisomerases 
(Gustafsson et al., 2016). A minimal replisome is however designated as PolG, mtSSB and TWINKLE owing to its ability to replicate mtDNA in vitro (Korhonen et al., 2004). Although the mtDNA replisome is known, it is rather the mode of replication that has proved controversial over the years. Three models of mtDNA replication have been proposed: the original strandasynchronous model, the alternative strand-synchronous model, and the unidirectional replication model. The strand-asynchronous model suggests an L-strand transcript, which is processed by the mtDNA replisome into primers for the $\mathrm{H}$-strand, primes mtDNA replication. Following about $70 \%$ of $\mathrm{H}$-strand replication and $\mathrm{H}$-strand displacement, the origin of $\mathrm{L}$-strand $\left(\mathrm{O}_{\mathrm{L}}\right.$, see Figure 10$)$ is exposed and $\mathrm{L}$ strand replication begins and proceeds in the opposite direction (Shadel and Clayton, 1997; Wong and Clayton, 1985). The strand-synchronous model posits that both $\mathrm{H}$ and $\mathrm{L}$ strands are synthesized bidirectionally from within an area in the noncoding region beyond the displacement loop (D-loop, see Figure 10) (Holt et al., 2000). The third model proposes that mtDNA replication starts at either one of the two sites (consistent with the asynchronous model). DNA synthesis progresses in the leading strand with simultaneous RNA incorporation throughout the lagging strand (RITOLS) (Yasukawa et al., 2006).

\subsubsection{Mitochondrial biogenesis}

\subsubsection{Transcriptional regulation of mitochondrial biogenesis}

Mitochondrial biogenesis, which serves to increase the mass and copy number of mitochondria, and increase mitochondrial metabolic capacity, is a process that requires the coordinated and regulated expression of nuclear and mitochondrial genomes. Although only 13 of such proteins are encoded by the mtDNA, they are nevertheless essential because they form the core of most complexes of the respiratory chain, and oxidative phosphorylation (OXPHOS) collapses when they are not expressed (Larsson et al., 1998). Most mitochondrial proteins (about 99\%), including the factors that drive mtDNA expression, are encoded by nuclear genes, synthesized on cytosolic ribosomes and imported into mitochondria. Nuclear control of mitochondrial biogenesis was originally found to be driven by nuclear respiratory factors, NRF1 and NRF2 (Evans and Scarpullas, 1989; Virbasius et al., 1993b). NRF1 and NRF2 regulate the expression of distinct classes of mitochondrial proteins. These include proteins, which drive mtDNA transcription and replication, several subunits of the respiratory chain complexes, cytosolic enzymes of heme synthesis and mitochondrial protein import components (Virbasius et al., 1993a, 1993b). In addition to the NRFs, several other factors, including the peroxisome 
proliferator-activated receptor (PPAR) $\alpha$ and $\delta$ and estrogen-related receptor $\alpha(E R R \alpha)$, target mitochondrial function by mediating the expression genes of the $\beta$-oxidation of fatty acids pathway (Gulick et al., 1994; Schreiber et al., 2004).

Several transcriptional regulators have been shown to mediate mitochondrial biogenesis (see review in (Scarpulla, 2008)). Integrating the actions of these multiple regulators suggests the activity of an overarching control factor. This was provided by the discovery of the PPARY

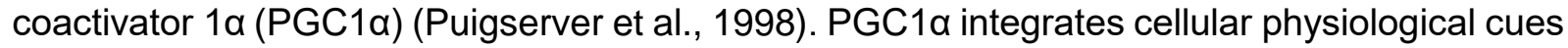
like thermogenesis, cell growth, and gluconeogenesis in its coordination of the activity of several transcriptional regulators of mitochondrial biogenesis (Lin et al., 2005). Subsequently, another PGC1 family of coactivators, PGC1 $\beta$ and PGC1-related coactivator (PRC) has been discovered. These factors, just like PGC1 1 , mediate gene expression through transcriptional regulators or directly through the targets of these transcription factors (Lin et al., 2005; Scarpulla, 2008).

\subsubsection{Mitochondrial protein import}

Nuclear-encoded mitochondrial transcripts, whose expression is induced by the transcriptional regulators described above, are translated on cytosolic ribosomes into precursor proteins with targeting sequences directing them to the mitochondrion. The precursor proteins are stabilized by Hsp90 and Hsp70 and delivered to the import receptor Tom70 (Young et al., 2003). The protein import machinery of mitochondria imports and sorts the precursor proteins via at least four distinct pathways (Chacinska et al., 2009). Almost all precursor proteins destined for the mitochondria are imported through the translocase of the outer membrane (TOM complex), which consists of Tom20, Tom22 and Tom70 (receptor subunits), which recognize precursor proteins and translocate them through the channel-forming Tom40 subunit. The other subunits of the TOM complex are Tom5, Tom6, and Tom7 (small subunits), which are involved in assembly, stability and dynamics of the TOM complex (Rapaport, 2005; Sherman et al., 2005).

Following translocation through the TOM complex, depending on the structure and final destination, each precursor protein is sorted via one of at least four pathways. The TOM complex assists in the insertion of the simplest of outer membrane proteins into the OMM (Rapaport, 2005). OMM proteins typified by complex topology such as porins are initially delivered into the IMS. These proteins transiently associate with the Tim9-Tim10 chaperone complex and are delivered to the sorting and assembly machinery (SAM), which mediates their insertion into the OMM (Paschen et al., 2003; Wiedemann et al., 2003). Import of precursor 
proteins targeted to the IMS, IMM, and matrix involves the translocase of inner membrane (TIM complex), which includes two distinct complexes TIM23 and TIM22. From the TOM complex, precursor proteins are directed to the TIM23 complex, which comprises Tim23, Tim50, Tim17, Tim21, and Tim44. Tim50 recognizes the precursor proteins and translocates them through the channel-forming Tim23 subunit (Mokranjac and Neupert, 2005).

Translocation through the TIM23 complex into the matrix or insertion into the inner membrane requires membrane potential. IMM-destined precursor proteins are arrested by their hydrophobic stop signals and are laterally released into the IMM (Wiedemann et al., 2004). In addition, the TIM23 complex associates with the presequence translocase-associated motor (PAM), while Tim44 recruits ATP-bound mtHSP70 from the matrix. Import motor-driven ATP hydrolysis stabilizes mtHSP70 interactions with Tim44 and with matrix precursor proteins thus preventing their retrograde movement into the IMS. ADP exchange by a new ATP, mediated by nucleotide exchange factor Mge1, facilitates precursor protein release into the matrix (Chacinska et al., 2009; Mokranjac and Neupert, 2005; Wiedemann et al., 2004).

The second TIM complex, TIM22 mediates the insertion of carrier proteins into the IMM. TIM22 complex is composed of Tim22 (shares homology with Tim23), Tim54, Tim18 and small Tims. The Tim9-Tim10 chaperone complex in the IMS binds precursor carrier proteins, following their translocation through the TOM complex and delivers them to the TIM22 complex by directly docking to it. The precursor proteins are translocated through the channel-forming protein Tim22 and laterally released into the lipid phase of the IMM in a membrane potential-dependent manner (Rehling et al., 2003; Wiedemann et al., 2004).

Mitochondrial proteins targeted to the IMS including the small Tims, which act as chaperone complexes for nascent mitochondrial proteins, are mostly small.A dedicated import pathway for these proteins has been described: the mitochondrial IMS import and assembly (MIA) pathway. The import mechanisms in this pathway have been reviewed excellently in Chatzi et al. (2016) and Stojanovski et al. (2008).

While these pathways mediate the import of nuclear-encoded mitochondrial proteins, the oxidase assembly (OXA) translocase exports mtDNA-encoded respiratory chain proteins into the IMM. In addition, the OXA translocase mediates the export of a small fraction of nuclearencoded mitochondrial proteins, which are initially targeted into the matrix via the TIM23 complex (Hell et al., 1998; Stiller et al., 2016). 


\subsection{Mitochondrial metabolism}

The oxidation of glucose via glycolysis releases only a small fraction of the potential free energy contained in glucose. Most of the energy in eukaryotic cells is derived from complete oxidation of carbohydrates and fatty acids in mitochondria. The mitochondrion houses several metabolic pathways, whose enzymes are distinctly localized to the IMM or to the matrix. The enzymatic machinery of oxidative phosphorylation is found in the IMM while those of carbohydrate, lipid and amino acid oxidation are localized to the matrix. In addition, enzymes of the heme biosynthetic pathway and some enzymes of the urea cycle are found in the matrix.

\subsubsection{Generation of ATP}

\subsubsection{Glycolysis}

ATP is generated via a series of redox reactions and involves the transport of energy from chemical bonds to high-energy compounds. Glucides, especially glucose, and fatty acid oxidation serve as the main source of energy, although amino acids and ketone bodies can also be oxidized for ATP generation under starvation conditions. The process of glucose oxidation to pyruvate, termed glycolysis, is catalyzed by cytosolic enzymes. The 10 reactions of the glycolytic pathway generate pyruvate and NADH in the cytosol with a net gain of two ATP molecules per glucose molecule. In the absence of oxygen $\left(\mathrm{O}_{2}\right)$, pyruvate oxidizes NADH, yielding lactate in mammalian cells. However, under aerobic conditions, pyruvate is transported into the mitochondrial matrix, where it is decarboxylated to acetyl-Coenzyme A (CoA) with simultaneous generation of NADH. Acetyl-CoA enters the tricarboxylic acid cycle.

\subsubsection{The tricarboxylic acid cycle}

The enzymes, which catalyze the reactions of the tricarboxylic acid (TCA) cycle, are localized to the mitochondrial matrix or to the matrix side of the IMM (succinate dehydrogenase). Following the demonstration by Franz Koop and Carl Martinus of a series of reactions that yielded oxaloacetate from citrate, Hans Krebs and William Johnson discovered the cyclical nature of the reactions from citrate oxidation to oxaloacetate. 


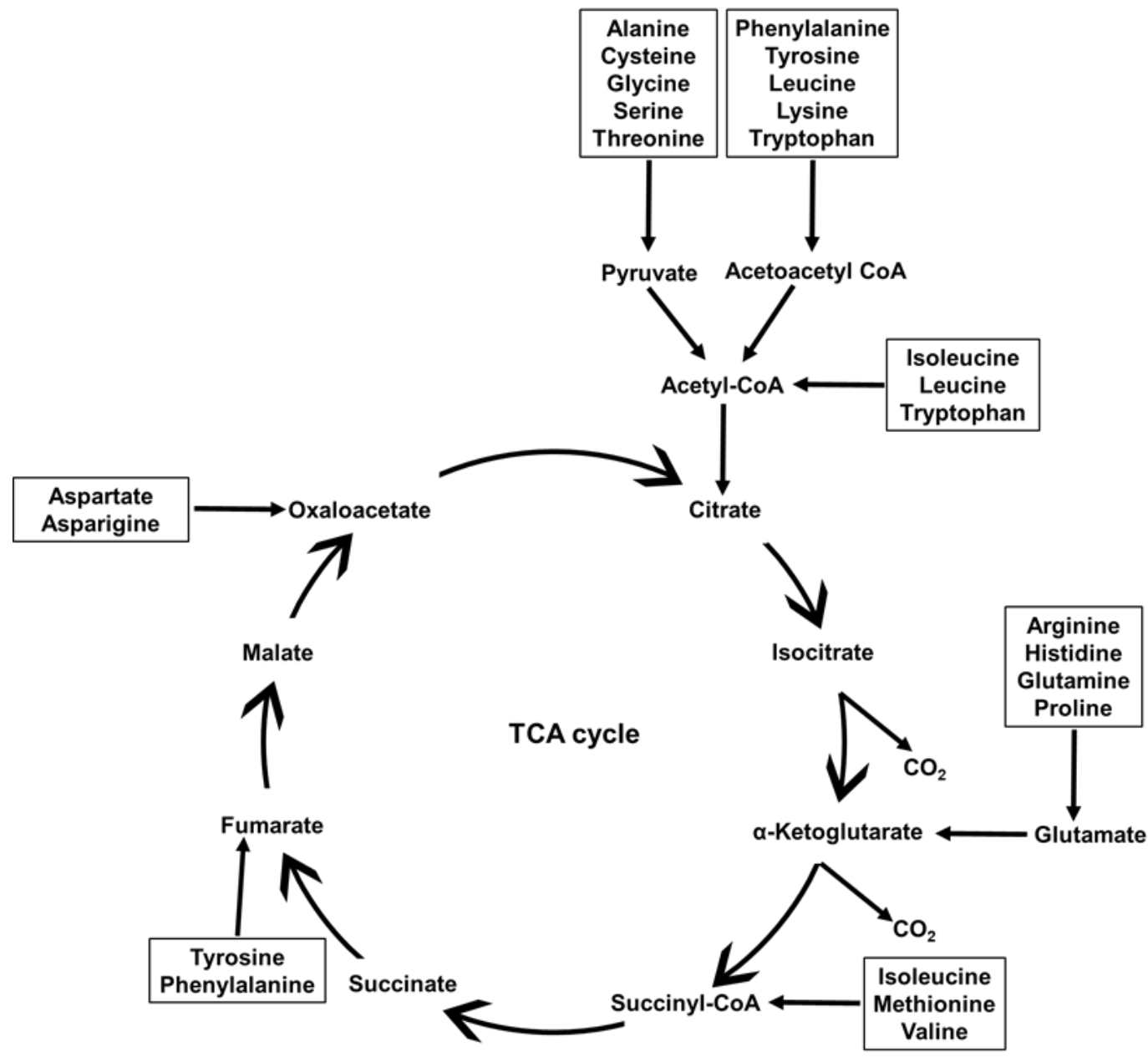

Figure 11| Schematic representation of the TCA cycle - The TCA cycle as illustrated above also depicts points of entry of Acetyl-CoA and amino acids into the cycle. Enzymes that catalyze each step of the reactions, as well as the reverse reactions in the TCA cycle, are not indicated. (original illustration, KF Yambire)

They also filled the gaps in the cycle while proposing a two-carbon donor, which allowed the conversion of oxaloacetate to citrate (Krebs and Johnson, 1937). It would take more than a decade until the two-carbon donor was discovered to be acetyl-CoA (Lipmann et al., 1950). The TCA cycle, as illustrated in Figure 11, completely oxidizes acetyl-CoA into two molecules of $\mathrm{CO}_{2}$ while concurrently conserving the liberated energy as high-energy compounds, three molecules of $\mathrm{NADH}$ and one molecule of $\mathrm{FADH}_{2}$ per molecule of acetyl-CoA oxidized. These high-energy compounds are subsequently used as substrates for mitochondrial oxidative phosphorylation to generate ATP. 


\subsubsection{Respiratory chain and oxidative phosphorylation}

The process of ATP synthesis via oxidative phosphorylation requires the generation of an electrochemical gradient across the IMM, which is used by the ATP synthase to catalyze the reaction, $\mathrm{ADP}+\mathrm{Pi} \rightarrow \mathrm{ATP}$. The electrochemical gradient is generated and maintained by the mitochondrial respiratory chain, which consists of four enzyme complexes, denoted complexes I, II, III and IV. These complexes, in addition to the ATP synthase (complex V), are localized to the IMM. Peter Mitchell proposed and demonstrated this process of coupling respiration to ATP synthesis in mitochondria, referred to as the chemiosmotic theory (Mitchell, 1961), which is widely accepted today.

The operation of the enzyme complexes of the respiratory chain is well characterized. Complex I catalyzes the transfer of two electrons from NADH to ubiquinone to form ubiquinol while simultaneously pumping four protons into the IMS. Several iron-sulphur clusters mediate the electron transfer by complex I. Electron transfer from succinate through iron-sulfur clusters and heme to FAD and then to ubiquinone is mediated by complex II, which lacks proton-pumping activity. Complex III then catalyzes the transfer of electrons from ubiquinol to cytochrome $\mathrm{c}$ via the $Q$ cycle. Four protons are concurrently pumped into the IMS by complex III for every pair of electrons transferred. The terminal enzyme complex of the respiratory chain, complex IV, catalyzes the transfer of electrons from reduced cytochrome $c$ to oxygen to generate water. Translocation of four protons by complex IV into the IMS is coupled to every pair of electrons transferred. Finally, the ATP synthase utilizes the proton motive force generated by the respiratory chain complexes I, III and IV to catalyze ATP synthesis. This process is also coupled to proton translocation from the IMS into the matrix. Details of the mechanisms of electron transfer and oxidative phosphorylation are described in Scheffler (2007).

\subsection{Mitochondria in metabolic signaling}

The nucleus is typically viewed as the 'control center' of the cell, the compartment where physiological cues are integrated to command diverse cellular processes including directing mitochondrial function through mitochondrial biogenesis. Given the crucial role of mitochondria in driving several metabolic processes especially in ATP production, it is rational to think that optimal cellular function would require the integration of functional cues from mitochondria to the rest of the cell. This is further highlighted by the diverse nature of mitochondrial diseases, which seem to be of multiple etiologies other than just a decline in ATP levels. Mitochondrial signaling has been coined to describe such mechanisms of communication between mitochondria and the rest of the cell. 
Reactive oxygen species (ROS) are generated by complexes I and III of the respiratory chain. Several lines of evidence suggest that mitochondrial ROS (mROS) regulate signaling pathways in the rest of the cell. For instance, mROS have been shown to regulate cellular adaptation to hypoxia. Because oxygen is required for ATP synthesis by mitochondria, hypoxic conditions trigger hypoxia-inducible factors, which induce the expression of EPO and VEGF and several glycolytic enzymes. This response serves to boost oxygen availability and maintain ATP levels. Bell et al. (2007) demonstrated in cells lacking complex III subunit, cytochrome b, that HIF-1a stabilization under hypoxic conditions was dependent on mROS and not on oxidative phosphorylation. mROS have been implicated in the regulation of proliferation and differentiation (Nunnari and Suomalainen, 2012). Furthermore, Raimundo et al. (2012) showed that mROS-dependent activation of AMPK in transgenic-mtTFB1 mice induced E2F1-mediated loss of inner ear ganglion neurons.

For a very long time, metabolites used to be considered just by-products of metabolic reactions mostly driven by mitochondrial enzymes. This view has been challenged by increasing reports of their role in modulating several cellular processes. The TCA cycle intermediate, $\alpha$ ketoglutarate, for example, has been shown to increase lifespan in $C$. elegans, in a similar way to caloric restriction, by inhibiting ATP synthase and TOR while inducing autophagy (Chin et al., 2014). In addition, succinate and fumarate, two TCA cycle metabolites, induce oncogenesis by facilitating angiogenesis, metastasis, and glycolysis (Pollard et al., 2005; Selak et al., 2005). A detailed description of metabolites and other signaling molecules from mitochondria is discussed in several excellent reviews (Hamanaka and Chandel, 2010; Nunnari and Suomalainen, 2012; Raimundo, 2014; Raimundo et al., 2011).

The electrochemical gradient, which provides the driving force for ATP synthesis is also coupled to the handling of ions with signaling functions by mitochondria. The most characterized of these ions is $\mathrm{Ca}^{2+}$. Mitochondrial $\mathrm{Ca}^{2+}$ handling is based on three cooperating modules: $\mathrm{Ca}^{2+}$ uptake from the non-mitochondrial compartments by the mitochondrial uniporter (MCU), its buffering in the mitochondrion and its efflux into the cytosol through the $\mathrm{Na}^{+} / \mathrm{Ca}^{2+}$ exchanger to drive cellular processes. All these processes are dependent on the electrochemical gradient generated by the respiratory chain (Rizzuto et al., 2012). Although mitochondrial $\mathrm{Ca}^{2+}$ uptake is known to drive local metabolic processes such as increasing ATP production (Jouaville et al., 1999) or regulating mitochondrial fission through DRP1 phosphorylation (Cribbs and Strack, 2007), its role in regulating diverse extramitochondrial cellular processes has also been established. Mitochondrial $\mathrm{Ca}^{2+}$ uptake, for example, has been shown to affect cell death pathways such as necrosis and apoptosis. In excitatory 
neurons, NMDA receptor activation, for instance, results in increased $\mathrm{Ca}^{2+}$ conductance through the NMDA receptor, as well as through voltage-gated $\mathrm{Ca}^{2+}$ channels, which open following depolarization. Mitochondria have been shown to accumulate $\mathrm{Ca}^{2+}$ under these conditions thereby buffering the cytosolic load. However, prolonged influx of $\mathrm{Ca}^{2+}$ coupled with decreased mitochondrial buffering capacity and decreased cytoplasmic $\mathrm{Ca}^{2+}$ clearance mechanisms initiate necrosis (Bano et al., 2005). In addition, the electrochemical gradient dissipates following mitochondrial $\mathrm{Ca}^{2+}$ overload resulting in necrosis induction.

The release of pro-apoptotic factors such as cytochrome $\mathrm{c}$ by mitochondria is implicated in apoptosis. Mitochondrial $\mathrm{Ca}^{2+}$ mediates the activity of the permeability transition pore, whose prolonged opening results in cytochrome c release and apoptosis initiation (Rasola and Bernardi, 2011). Furthermore, mitochondrial $\mathrm{Ca}^{2+}$ handling affects autophagy, a pathway that modulates cell survival. Increased inositol 1,4,5-triphosphate (IP3) receptor-mediated mitochondrial $\mathrm{Ca}^{2+}$ signaling was shown to regulate AMP-activated protein kinase (AMPK) activity, a positive regulator of autophagy (Cárdenas et al., 2010).

\subsection{Mitochondria in immune response pathways}

Induction of the innate immune response is initiated by pattern recognition receptors (PRRs), which recognize pathogen-associated molecular patterns (PAMPs) on invading pathogens. Ligation of PRRs triggers downstream signaling mediated by nuclear factor $k B(N F k B)$, mitogen-activated protein kinases (MAPKs) and interferon regulatory factors (IRFs), which induce the expression of cytokines and chemokines and type I interferons to contain the pathological insult while triggering the adaptive immune response (West et al., 2011). Following the discovery of Toll-like receptors (TLRs), several families of cytosolic PRRs were found, including retinoic acid-inducible gene I (RIG-I)-like receptor (RLRs), nucleotide oligomerization domain (NOD)-like receptors (NLRs), and C-type lectin receptors (CLRs) (Kawai and Akira, 2010; West et al., 2011).

Mitochondria were previously known to play a role in immune response through the induction of apoptosis following infection. However, multiple lines of evidence suggest that mitochondrial involvement in the immune response goes beyond apoptosis induction. The discovery of the mitochondrial antiviral signaling protein (MAVS) as an adaptor protein for RLRs provided evidence of mitochondrial involvement in immune response induction (Kawai et al., 2005; Meylan et al., 2005; Seth et al., 2005; Xu et al., 2005). Interestingly, MAVS was found to localize to the OMM where it recruits RLRs following viral infection (Seth et al., 2005). In addition, MAVS 
was found to mediate downstream signaling by interacting with tissue necrosis factor (TNF) receptor-associated factor (TRAF) family members. Strikingly, MAVS loses its signaling capacity when mislocalized to the cytosol (Seth et al., 2005).

Furthermore, mROS has been shown to promote antibacterial innate immune response and bactericidal activity. Evidence of the role of mROS in immunity was first reported in mice lacking the mitochondrial uncoupler protein 2 (UCP2). These mice were challenged with Toxoplasma gondii and were found to be more resistant to Toxoplasma-induced lethality when compared to their wild-type littermates (Arsenijevic et al., 2000). Macrophages from these mice showed increased bactericidal activity and higher basal and Toxoplasma-induced mROS levels (Arsenijevic et al., 2000). Conversely, macrophages overexpressing UCP2 produced lower levels of cellular ROS when stimulated with TLR4 agonist lipopolysaccharide (Kizaki et al., 2002), thus demonstrating that mROS contribute to the proper induction of the innate immune response.

Lastly, sterile immune responses, unlike infections, are triggered by PRR-dependent sensing of damage-associated molecular patterns (DAMPs) following cellular injury. TLRs and NLRs mediate the sensing of DAMPs and the subsequent induction of the immune response. A well characterized mitochondrial DAMP is the hypomethylated CpG motifs of mtDNA, which resemble bacterial CpG DNA. mtDNA is thus a potent agonist of TLR9- mediated induction of the innate immune system (discussed earlier, see section 1.3.3.3).

\subsection{Lysosomal and mitochondrial crosstalk}

The endosymbiotic theory of organelle acquisition has been widely accepted as defining the evolution of eukaryotes. Although this theory is consistent with the first kind of organelle crosstalk between the primitive lysosome (phagosome) and the ancestral mitochondrion, a more entrenched position by some endosymbiont proponents was the view that modern organelles should be as autonomous as their ancestors (Bogorad, 1975). This view is certainly challenged by mounting evidence, which suggests that organelles exist as interdependent units in the eukaryotic cell.

We have recently reviewed the communication between mitochondria and other membranebound compartments of the cell (Diogo et al., 2017; Raimundo et al., 2016). Nevertheless, in the context of this study, recent evidence, which pertains to the crosstalk between lysosomes and mitochondria, which may involve physical interactions or via signaling pathways 
(Raimundo, 2014), will be discussed. The most notable of cellular processes that link lysosomes and mitochondria, is the selective degradation of damaged mitochondria by lysosomes, known as mitophagy (Youle and Narendra, 2011). Classical mitophagy is mediated by PINK1 and Parkin (whose genes are mutated in autosomal recessive Parkinson's disease). Several mechanisms involved in mitophagy have been characterized and discussed in several reviews (Pickrell and Youle, 2015; Whitworth and Pallanck, 2017; Youle and Narendra, 2011). One of the most hotly discussed mechanisms involves PINK and Parkin. Briefly, in healthy, TIM import-competent mitochondria, the kinase PINK1 is targeted to the mitochondrial matrix for processing and degradation. Following IMM depolarization in damaged mitochondria, PINK1 import is blocked and it accumulates on the OMM where it recruits the cytosolic E3 ubiquitin ligase Parkin to the mitochondrial surface. Parkin ubiquitinates several target proteins on the OMM. Ubiquitination of OMM proteins results in the recruitment of ubiquitin adaptor proteins, which promote the engulfment and sequestration of damaged mitochondria into autophagosomes and targeted to the lysosome for degradation.

Furthermore, contact sites between mitochondria and vacuole were recently reported in yeast (Elbaz-Alon et al., 2015), although evidence of such interactions in mammalian cells is still lacking. Another striking finding pertaining to mitochondrial and lysosomal crosstalk was recently reported by Heidi McBride and colleagues. In this report, the authors showed in mammalian cells that under subtoxic doses of ROS, cargo-containing mitochondrial-derived vesicles (MDVs) budded from mitochondria and delivered their cargo to lysosomes (Soubannier et al., 2012). Although this work illustrates an essential mitochondrial quality control pathway, it remains unclear whether it applies in vivo.

In addition, we and others have demonstrated recently that mitochondria and lysosomes communicate via signaling pathways (Baixauli et al., 2015; Fernández-Mosquera et al., 2017). We showed in cells that acute mitochondrial malfunction, induced by mitochondrial poisons, triggered TFEB/MITF-dependent lysosomal biogenesis via AMPK (Fernández-Mosquera et al., 2017). The existence of an interdependent network between these compartments is further highlighted by several reports of mitochondrial defects in LSDs and of lysosomal malfunction in mitochondrial disorders (reviewed in Diogo et al., 2017; Ordonez, 2012; Osellame and Duchen, 2014; Raimundo et al., 2016). For example, mitochondrial defects including impaired respiration, reduced respiratory chain enzyme activities, decreased membrane potential, and fragmentation were reported in a mouse model of Gaucher's disease, the most common LSD (Osellame et al., 2013). Interestingly, Gaucher's disease is associated with increased risk of Parkinson's disease. Finally, chronic mitochondrial malfunction, akin to most mitochondrial 
diseases, was shown to result in the accumulation of swollen and dysfunctional lysosomes in fibroblasts from mice lacking OPA1, PINK1 or AIF (Demers-Lamarche et al., 2016). The crosstalk between lysosomes and mitochondria likely plays a crucial role in the pathogenesis of metabolic diseases and understanding the mechanisms involved should be of utmost priority.

\subsection{Aims of the study}

Mitochondrial malfunction has been shown in several lysosomal storage disorders (LSDs). However, the mechanism(s) underlying mitochondrial defects in LSDs remain virtually unknown. The aims of this study were to identify the mechanistic links between LSDs and mitochondrial malfunction. Given that distinct etiologies including mutations in different groups of hydrolases or permeases underlie the different LSDs, it is anticipated that different mechanisms might mediate mitochondrial dysfunction in different LSDs. That notwithstanding, it is also expected that common mechanisms linking different LSDs to mitochondrial malfunction may exist due to secondary lysosomal enzyme deficiencies that have been reported in several LSDs. In addition, it was interesting to know the role that the identified mechanisms of lysosomal and mitochondrial crosstalk played in the pathogenesis of lysosomal storage disorders. Importantly, this study also sought to harness the therapeutic potential of mechanistic links between lysosomes and mitochondria to mitigate disease consequences. To this end, the specific goals of this study were to:

- Identify the mechanism(s) of mitochondrial malfunction in cellular and mouse models of Niemann-Pick disease Types A/B/C (lipid storage disorders).

- Demonstrate central nervous system pathology in mouse models of Pompe's disease (glycogen storage disorder) and elucidate the mechanism(s) that links lysosomal malfunction in Pompe's disease to mitochondrial dysfunction.

- Show whether the identified mechanism(s) could be modulated pharmacologically to ameliorate disease outcomes. 


\section{Materials and methods}

\subsection{Materials}

\subsubsection{Reagents}

Several chemicals and reagents were used in this study. The respective lists of reagents and their suppliers are provided in the table below.

Table 1: List of reagents and their suppliers and catalog numbers

\begin{tabular}{lll}
\hline \hline Product & Supplier & Catalog number \\
\hline Absolute ethanol & BD Bioscience & 354052 \\
Acrylamide solution 40\% & AppliChem & A0385 \\
Agarose & Carl Roth & 3810.3 \\
Ammonium Persulfate (APS) & AMRESCO & 0486 \\
Antimycin & Sigma Aldrich & A8674 \\
Bovine serum albumin (BSA) & Sigma Aldrich & A7906 \\
Bromophenol blue & Carl Roth & T116.1 \\
CaCl 2 & Alfa Aesar & L13191 \\
CCCP & Fluka & 857815 \\
Chloral hydrate & Sigma Aldrich & C8383 \\
Chloroquine disphosphate & Sigma Aldrich & C6628 \\
Cold fish gelatin & Sigma Aldrich & G7765 \\
Coomassie Brilliant Blue G & Carl Roth & 9598.2 \\
Cryo-OCT compound & Fisher Scientific & $14-373-65$ \\
Cytochrome c (from bovine heart) & Sigma Aldrich & C2037 \\
D(+)-Glucose & Carl Roth & 275227941 \\
DAPI & Carl Roth & C6335.1 \\
Deferoxamine mesylate & Sigma Aldrich & D9533 \\
Desipramine & Biotrend & BG0162 \\
Diaminobenzidine & Sigma Aldrich & D8001 \\
Isoflurane & Sigma & 792632 \\
Digitonin & Merck & $11024-24-1$ \\
\hline
\end{tabular}


Table 1: Continued from previous page

\begin{tabular}{|c|c|c|}
\hline Product & Supplier & Catalog number \\
\hline DMEM High glucose & Gibco & $41965-062$ \\
\hline DMSO & Sigma Aldrich & D8418 \\
\hline DTT & AppliChem & A2948 \\
\hline $\begin{array}{l}\text { Dulbecco's Modified Eagle's Medium } \\
\text { (DMEM) Base } 8.3 \mathrm{~g} / \mathrm{L}\end{array}$ & Sigma Aldrich & D5030-1L \\
\hline$\varepsilon$-Amino n-caproic acid & Merck & CAS6032-2 \\
\hline EDTA & Carl Roth & 8043.2 \\
\hline Ethanol $70 \%$ & VWR & 84858.440 \\
\hline Ethidium bromide & VWR & E406 \\
\hline FCCP & Sigma Aldrich & C2920 \\
\hline Ferric citrate & Sigma Aldrich & F3388 \\
\hline Folic acid & Sigma Aldrich & F8758 \\
\hline GlutaMax-1 (100X) & Gibco & $35050-061$ \\
\hline Glycerol & Carl Roth & 7530.1 \\
\hline Glycine & VWR & 0167 \\
\hline Goat Serum & Life Technologies & $10000 \mathrm{C}$ \\
\hline $\mathrm{H}_{2} \mathrm{O}$ Nuclease-free & VWR & E476 \\
\hline $\begin{array}{l}\text { Halt }{ }^{\mathrm{TM}} \text { Protease \& Phosphatase single use } \\
\text { Inhibitor Cocktail }\end{array}$ & Thermo Scientific & 78442 \\
\hline $\mathrm{HCl}$ & Sigma Aldrich & $\mathrm{H} 1758$ \\
\hline Heparin & Sigma Aldrich & H3393 \\
\hline HEPES & Carl Roth & HN77.2 \\
\hline $\mathrm{K}_{2} \mathrm{HPO}_{4}$ & Carl Roth & P749.2 \\
\hline $\mathrm{KCl}$ & Volu-Sol & 83608.26 \\
\hline $\mathrm{KH}_{2} \mathrm{PO}_{4}$ & Carl Roth & 3904.1 \\
\hline 2-Mercaptoethanol & Carl Roth & 4227.3 \\
\hline Methanol & VWR & 20903.368 \\
\hline $\mathrm{MgCl}_{2}$ & VWR & 8.14733 .0100 \\
\hline $\mathrm{MgSO}_{4}-7 \mathrm{H}_{2} \mathrm{O}$ & Sigma Aldrich & 63138 \\
\hline
\end{tabular}


Table 1: Continued from previous page

\begin{tabular}{lll}
\hline Product & Supplier & Catalog number \\
\hline Mowiol 4-88 & AppliChem & A9011 \\
$\mathrm{NaCl}$ & AppliChem & A1430,0010 \\
$\mathrm{NaH}_{2} \mathrm{PO}_{4} \cdot \mathrm{H}_{2} \mathrm{O}$ & Carl Roth & K300.1 \\
$\mathrm{NaHCO}_{3}$ & Carl Roth & P029.3 \\
$\mathrm{NaN}_{3}$ & Sigma Aldrich & $52002-1006$ \\
$\mathrm{~N}$-dodecylmaltoside & Carl Roth & CN26.2 \\
$\mathrm{NH}_{4} \mathrm{Cl}$ & Carl Roth & K298.1 \\
Nonidet@P-40 Substitute & AMRESCO & E109-50ML \\
Oligomycin & Sigma Aldrich & O4876 \\
Paraformaldehyde (PFA) & AppliChem & A3813 \\
PMSF & Sigma & P7626 \\
Polybrene & Sigma & $28728-55-4$ \\
Proteinase K & Amresco & 0706 \\
Puromycin 100mg & Fisher Scientific & BP2956-100 \\
Ribonuclease A & Amresco & 0675 \\
Rotipherose Gel 40 (37.5:1) & Carl Roth & T802.1 \\
SDS & Sigma Aldrich & L4509-500G \\
Sew2871 & Cayman & 10006440 \\
Skim Milk Powder & Fluka & 70166 \\
Sodium deoxycholate & Sigma Aldrich & 30970 \\
TEMED & Sigma Aldrich & T7024 \\
Torin-1 & BioVision & 2273 \\
Trehalose & Carl Roth & 5151.3 \\
TRI Reagent & Sigma & T9424 \\
Tris Base & Sigma Aldrich & T1503 \\
Tris-HCl & Carl Roth & 9090.2 \\
Triton X-100 & AMRESCO & 0694 \\
Tween 20 & AMRESCO & $0777-1 \mathrm{~L}$ \\
U0126 & & 662005 \\
\hline \hline & & \\
\hline
\end{tabular}




\subsubsection{Kits and consumables}

The kits and consumables used in this thesis are listed in Table 2 below. The corresponding suppliers and catalog numbers of the kits are also provided.

Table 2: List of kits and consumablesables used in the study

\begin{tabular}{|c|c|c|}
\hline Product & Supplier & Catalog number \\
\hline Amersham Hybond PO45 PVDF & GE Healthcare & 10600023 \\
\hline $\begin{array}{l}\text { Centrifuge tubes } 15 \mathrm{~mL} \text { Corning } \\
\text { CentriStar }\end{array}$ & Corning & 430791 \\
\hline $\begin{array}{l}\text { Centrifuge tubes } 50 \mathrm{~mL} \text { Corning } \\
\text { CentriStar }\end{array}$ & Corning & 430829 \\
\hline Cryomold (tissue tek) & Fisher Scientific & NC9511236 \\
\hline CRYSTAL RNA Mini Kit & New England Biolabs & $31-010-404$ \\
\hline Cuvettes PMMA & VWR-International & $634-0678$ \\
\hline $\mathrm{DQ}^{\mathrm{TM}}$ Red BSA & Life Technologies & D12051 \\
\hline $\begin{array}{l}\text { FACS Tubes Polystyrene Round-Bottom } \\
5 \mathrm{~mL}\end{array}$ & Corning & 352052 \\
\hline Fetal Bovine Serum Heat-Inactivated & Gibco & $10500-064$ \\
\hline Fugene R 6 Transfection Reagent & Promega & E2691 \\
\hline GoTaq Flexi DNA polymerase kit & Promega & M8291 \\
\hline $\begin{array}{l}\text { High-Performance chemiluminescence } \\
\text { film }\end{array}$ & GE Healthcare & 28906837 \\
\hline iScrip cDNA Synthesis Kit & Bio-Rad & $170-8891$ \\
\hline $\mathrm{iTaq}^{\mathrm{TM}}$ Universal SYBR Green Supermix & Bio-Rad & $172-5124$ \\
\hline Lenti $X$ concentrator & Clontech & 631231 \\
\hline Lipofectamine 2000 Reagent & Invitrogen & $11668-019$ \\
\hline
\end{tabular}

Continue on next page 
Table 2: Continued from previous page

\begin{tabular}{|c|c|c|}
\hline Product & Supplier & Catalog number \\
\hline $\begin{array}{l}\text { Luminata }{ }^{\mathrm{TM}} \text { Classico Western HRP } \\
\text { Substrate }\end{array}$ & Millipore & WBLUC0500 \\
\hline $\begin{array}{l}\text { Luminata }{ }^{\mathrm{TM}} \text { Crescendo Western HRP } \\
\text { Substrate }\end{array}$ & Millipore & WBLUC0500 \\
\hline $\begin{array}{l}\text { Luminata }{ }^{\mathrm{TM}} \text { Forte Western HRP } \\
\text { Substrate }\end{array}$ & Millipore & WBLUF0500 \\
\hline Medical X-Ray Film & Foma & ----- \\
\hline Microscope slides & Carl Roth & 2111 \\
\hline Microseal B seal & Bio-Rad & MSB1001 \\
\hline MitoSOXTM Red & Life Technologies & M36008 \\
\hline Mitotracker Deep Red & Life Technologies & L7526 \\
\hline Mitotracker Deep Red FM & Life Technologies & M22426 \\
\hline Mitotracker Green FM & Life Technologies & M7514 \\
\hline Native Gel marker & GE Healthcare & $17-0445-01$ \\
\hline $\begin{array}{l}\text { PageRuler Plus Prestained Protein } \\
\text { Ladder }\end{array}$ & Thermo Scientific & 26619 \\
\hline PBS tablets & Sigma Aldrich & P44177-100TAB \\
\hline $\begin{array}{l}\text { PCR plate 384-well skirted ABI-Type } \\
\text { (Universal) }\end{array}$ & StarLab & E1042-3840 \\
\hline Penicillin/Streptomycin & Gibco & $15140-062$ \\
\hline Pierce BCA Protein Assay Kit & Thermo Scientific & 23225 \\
\hline Protein Assay Dye Reagent Concentrate & Bio-Rad & $500-0006$ \\
\hline Serological Pipette $10 \mathrm{~mL}$ & StarLab & E4860-1011 \\
\hline Serological Pipette $25 \mathrm{~mL}$ & StarLab & E4860-2511 \\
\hline
\end{tabular}

Continue on next page 
Table 2: Continued from previous page

\begin{tabular}{lll}
\hline Product & Supplier & Catalog number \\
\hline Serological Pipette $5 \mathrm{~mL}$ & StarLab & E4860-0511 \\
Sodium Pyruvate & Sigma & S8636 \\
Test Tube Soda Glass & VWR-International & $212-003$ \\
Tips $10 \mu \mathrm{L}$ TipOne & StarLab & S111-3210 \\
Tips $1000 \mu \mathrm{L}$ TipOne & StarLab & S1111-6001 \\
Tips $20 \mu \mathrm{L}$ TipOne & StarLab & S120-1810 \\
Tips $200 \mu \mathrm{L}$ TipOne & StarLab & S1120-8800 \\
Triple ${ }^{\mathrm{t}}$ Express (1X) & Gibco & $12605-010$ \\
Trypan Blue 0.4\% & Life Technologies & T10282 \\
Tubes $0.5 \mathrm{~mL}$ molecular probes & Life Technologies & Q33856 \\
Tubes $1.5 \mathrm{~mL}$ & StarLab & E1415-1500 \\
Tubes $2 \mathrm{~mL}$ & StarLab & S1620-2700 \\
Tubes 8 Twin Strip Start PCR & StarLab & $11402-3700$ \\
Western Blot Paper & TH-Geyer & $4-01-60-0041$ \\
XFe 96 extracellular flux assay kit & BD Bioscience & $102416-100$ \\
Seahorse & & \\
\hline \hline
\end{tabular}




\subsubsection{Buffers and solutions}

All buffers and solutions that were used in this study are listed in Table 3 below. Unless otherwise stated, all buffers and solutions were prepared in ${ }_{d d} \mathrm{H}_{2} \mathrm{O}$ and concentrated buffers were further diluted to $1 \mathrm{X}$ in $\mathrm{dd}_{2} \mathrm{H}_{2} \mathrm{O}$ also.

Table 3: List of buffers and solutions

\begin{tabular}{|c|c|}
\hline Buffer/solution & Composition \\
\hline Anode buffer $10 x$ & 500mM Tris- $\mathrm{HCl}, \mathrm{pH} 7.0$ \\
\hline BN-loading dye $10 \mathrm{X}$ & $\begin{array}{l}5 \% \text { Coomassie blue, } 500 \mathrm{mM} \text { ع-Amino n-caproic acid, } \\
100 \mathrm{mM} \text { Tris, } \mathrm{pH} 7.0\end{array}$ \\
\hline Blocking buffer & $5 \%(w / v)$ Skimmed milk or BSA in TBST \\
\hline $\begin{array}{l}\text { Blocking buffer } \\
\text { (Immunohistochemistry) }\end{array}$ & $\begin{array}{l}0.1 \% \text { Cold fish gelatin, } 0.1 \% \text { Triton } \mathrm{X}-100,3 \% \mathrm{BSA}, 1 \% \\
\text { Goat serum and } 0.05 \% \text { Tween-20 in PBS }\end{array}$ \\
\hline Cathode Buffer & $500 \mathrm{mM}$ Tricine, $150 \mathrm{mM}$ Tris, $0.2 \%$ Coomassie G \\
\hline $\begin{array}{l}\text { Complex IV activity } \\
\text { staining buffer }\end{array}$ & $\begin{array}{l}50 \mathrm{mM} \mathrm{KPi} \text {, pH 7.4, 2.33mM 3, 3-diaminobenzidine, } 80 \mu \mathrm{M} \\
\text { reduced cytochrome c (bovine heart) }\end{array}$ \\
\hline DNA extraction buffer & $\begin{array}{l}50 \mathrm{mM} \text { Tris- } \mathrm{HCl} \mathrm{pH} 8.5,0.25 \% \text { SDS, } 1 \mathrm{mM} \text { EDTA and } \\
5 \mathrm{mM} \text { DTT }\end{array}$ \\
\hline $\begin{array}{l}\text { Digitonin solubilization } \\
\text { buffer }\end{array}$ & $\begin{array}{l}1 \% \text { Digitonin, } 20 \mathrm{mM} \text { Tris- } \mathrm{HCl}, \mathrm{pH} \text { 7.4, } 0.1 \mathrm{mM} \text { EDTA, } \mathrm{pH} \\
8.0,50 \mathrm{mM} \mathrm{NaCl}, 10 \% \text { glycerol, } 1 \mathrm{mM} \text { PMSF (freshly } \\
\text { added) }\end{array}$ \\
\hline \multirow[t]{2}{*}{ EBSS medium } & $\begin{array}{l}1.8 \mathrm{mM} \mathrm{CaCl}_{2}, 5.3 \mathrm{mM} \mathrm{KCl}, 117 \mathrm{mM} \mathrm{NaCl}, \quad 5.6 \mathrm{mM} \\
\text { Glucose, } 26 \mathrm{mM} \mathrm{NaHCO}_{3}, 0.8 \mathrm{mM} \mathrm{MgSO} 4 \text { and }\end{array}$ \\
\hline & $1 \mathrm{mM} \mathrm{NaH}{ }_{2} \mathrm{PO}_{4} \cdot \mathrm{H}_{2} \mathrm{O}$ \\
\hline Freezing medium & $10 \%(v / v)$ DMSO, $20 \%(v / v)$ FBS in DMEM high glucose \\
\hline Gel Buffer 3X & 0.2M ع-Amino n-caproic acid, $150 \mathrm{mM}$ Tris- $\mathrm{HCl}, \mathrm{pH} 7.0$ \\
\hline Homogenization buffer $A$ & $\begin{array}{l}300 \mathrm{mM} \text { Trehalose, } 10 \mathrm{mM} \mathrm{KCl}, 10 \mathrm{mM} \text { HEPES pH } 7.4 \\
\text { before use add freshly } 0.2 \% \text { BSA and } 1 \mathrm{X} \text { protease/ } \\
\text { phosphatase inhibitor cocktail }\end{array}$ \\
\hline Homogenization buffer B & $\begin{array}{l}\text { As Homogenization buffer A without } 0.2 \% \mathrm{BSA} \text { and } 1 \mathrm{X} \\
\text { protease/ phosphatase inhibitor cocktail }\end{array}$ \\
\hline Hypertonic buffer, pH 7.9 & $20 \mathrm{mM}$ Tris, $0.42 \mathrm{M} \mathrm{KCl}, 1.5 \mathrm{mM} \mathrm{MgCl}_{2}, 20 \%$ Glycerol \\
\hline Hypotonic buffer, pH 7.9 & $10 \mathrm{mM}$ Tris, $10 \mathrm{mM} \mathrm{KCl}$ and $1.5 \mathrm{mM} \mathrm{MgCl}_{2}$ \\
\hline Imaging buffer, $\mathrm{pH} 7.4$ & $\begin{array}{l}120 \mathrm{mM} \mathrm{NaCl}, 2.5 \mathrm{mM} \mathrm{KCl}, 2 \mathrm{mM} \mathrm{CaCl} \\
10 \mathrm{mM} \mathrm{HEPES}\end{array}$ \\
\hline
\end{tabular}

Buffers and solutions continued on next page 
Table 3: Continuation of list of buffers and solutions

\begin{tabular}{|c|c|}
\hline Buffer/solution & Composition \\
\hline Mowio|ß 4-88 & 6.4mM Mowiol 4-88, 5.4M Glycerol, 0.1M Tris pH 8.5 \\
\hline $\mathrm{NaN}_{3}$ & $10 \% \mathrm{NaN}_{3}$ \\
\hline PBS & Dissolve 5 PBS tablets in $1 \mathrm{~L}_{d d} \mathrm{H}_{2} \mathrm{O}$. Autoclave \\
\hline Resolving gel $12 \%$ & $\begin{array}{l}0.375 \mathrm{M} \text { Tris, } \mathrm{pH} \text { 8.8, } 0.1 \% \text { SDS, } 12 \% \text { Polyacrylamide, } \\
0.1 \% \text { APS, } 0.04 \% \text { TEMED }\end{array}$ \\
\hline RIPA buffer & $\begin{array}{l}50 \mathrm{mM} \text { Tris } \mathrm{pH} 8.0,150 \mathrm{mM} \mathrm{NaCl}, 1 \% \text { Triton } \mathrm{X}-100,0.1 \% \\
\mathrm{SDS} \text { and } 0.5 \% \text { Sodium deoxycholate supplemented with } \\
\text { fresh } 1 \mathrm{X} \text { protease/phosphatase inhibitor }\end{array}$ \\
\hline Running buffer $5 X$ & $125 \mathrm{mM}$ Tris Base, .96M Glycine, $.5 \%$ SDS \\
\hline SDS loading buffer & $\begin{array}{l}62.5 \mathrm{mM} \text { Tris, } \mathrm{pH} 6.8,50 \%(\mathrm{v} / \mathrm{v}) \text { Glycerol, } 12 \%(\mathrm{w} / \mathrm{v}) \\
\text { SDS, } 0.06 \%(\mathrm{w} / \mathrm{v}) \text { Bromophenol blue. Add } 5 \% 2- \\
\text { Mercaptoetanol freshly prior to use }\end{array}$ \\
\hline SNET buffer & $\begin{array}{l}20 \mathrm{mM} \text { Tris pH 8.0, 5mM EDTA pH 8.0, } 40 \mathrm{mM} \mathrm{NaCl}, 0.5 \% \\
\text { SDS }\end{array}$ \\
\hline Stacking gel $5 \%$ & $\begin{array}{l}62.5 \mathrm{mM} \text { Tris } \mathrm{pH} \text { 6.8, } 0.1 \% \text { SDS, } 5 \% \text { Polyacrylamide, } \\
0.1 \% \text { APS, } 0.1 \% \text { TEMED }\end{array}$ \\
\hline TAE buffer $50 \mathrm{X}$ & 2mM Tris, 50mM EDTA, $1 \mathrm{M}$ Glacial acetic acid pH 8.0 \\
\hline TBST 10X & $200 \mathrm{mM}$ Tris, $1.5 \mathrm{M} \mathrm{NaCl}, 1 \%$ Tween 20, pH 7.4 \\
\hline TE buffer & 10mM Tris-HCl, $0.2 \mathrm{mM}$ EDTA pH 7.5 \\
\hline Transfer buffer 10X & $\begin{array}{l}250 \mathrm{mM} \text { Tris, } 1.92 \mathrm{M} \text { Glycine. } 1 \mathrm{X} \text { dilution includes } \\
20 \%(\mathrm{v} / \mathrm{v}) \text { Methanol }\end{array}$ \\
\hline $\begin{array}{l}\text { Whole cell extraction } \\
\text { buffer }\end{array}$ & $\begin{array}{l}1.5 \% \mathrm{~N} \text {-dodecylmaltoside in PBS supplemented with } \\
\text { fresh } 1 \mathrm{X} \text { protease/phosphatase inhibitor }\end{array}$ \\
\hline $\begin{array}{l}\text { XF Seahorse assay } \\
\text { media }\end{array}$ & $\begin{array}{l}8.3 \mathrm{~g} \text { DMEM base, } 10 \mathrm{~mL} \text { Glutamax, } 10 \mathrm{~mL} \text { Sodium } \\
\text { pyruvate, } 1.85 \mathrm{~g} \mathrm{NaCl} \text { made up to } 1 \mathrm{~L} \text { with } \mathrm{dd}_{2} \mathrm{O} \text {. Sterile } \\
\text { filtered }\end{array}$ \\
\hline
\end{tabular}




\subsubsection{Primers}

All primers used for qPCR in this thesis were found in the Primer Bank repository of the Harvard Medical School and ordered from Integrated DNA Technologies. These primers are listed in Tables 4 and 5 below

Table 4: Human primers used for qPCR experiments in this study

\begin{tabular}{|c|c|c|}
\hline Primer & Sequence $\left(5^{\prime} \rightarrow 3^{\prime}\right)$-Forward & Sequence $\left(5^{\prime} \rightarrow 3^{\prime}\right)$-Reverse \\
\hline Atp6v0d1 & GAGGAACCATGCCTATGAGCC & GGATCACGTTGTCGATCATGTAA \\
\hline Atp6v0e1 & GTCCTAACCGGGGAGTTATCA & AAAGAGAGGGTTGAGTTGGGC \\
\hline Atp6v1a & GGGTGCAGCCATGTATGAG & TGCGAAGTACAGGATCTCCAA \\
\hline Atp6v1h & GCTGCTGTCCCCACCAATAT & GCTTCTCTTCAGGGCTTCGT \\
\hline Cox1 & AACCCAATACCAAACGCCCCT & GAAGGTGGTGTTGAGGTTGCG \\
\hline Cox10 & CACCGCCCAGCCTATCTTTG & TCATCTCTTTCCACCGCTTTTC \\
\hline Cox5a & ATCCAGTCAGTTCGCTGCTAT & CCAGGCATCTATATCTGGCTTG \\
\hline Ctsa & TCCCAGCATGAACCTTCAGG & AGTAGGCAAAGTAGACCAGGG \\
\hline Ctsb & ACAACGTGGACATGAGCTACT & TCGGTAAACATAACTCTCTGGGG \\
\hline Ctsc & CGATGTCAACTGCTCGGTTAT & AAGGCAAACCACTTGTAGTCATT \\
\hline Ctsd & ATTCAGGGCGAGTACATGATCC & CGACACCTTGAGCGTGTAG \\
\hline Ctsf & AGAGAGGCCCAATCTCCGT & GCATGGTCAATGAGCCAAGG \\
\hline Cytb & TACTCACCAGACGCCTCAACC & ATTGGCGTGAAGGTAGCGGAT \\
\hline Elk1 & AATCGGAAGAGCTTAATGTGGAG & CTTGGTGGTTTCTGGCACAAA \\
\hline Etv1 & TGGCAGTTTTTGGTAGCTCTTC & CGGAGTGAACGGCTAAGTTTATC \\
\hline Gaa & TGCCCTCGCAGTATATCACAG & GAGACCCGTAGAGGTTCGC \\
\hline Gapdh & GGAGTCAACGGATTTGGTCG & GACAAGCTTCCCGTTCTCAG \\
\hline Gba & ATGGAGCGGTGAATGGGAAG & GTGCTCAGCATAGGCATCCAG \\
\hline Hexa & ACGTCCTTTACCCGAACAACT & CGAAAAGCAGGTCACGATAGC \\
\hline Hprt & ACCAGTCAACAGGGGACATAA & CTTCGTGGGGTCCTTTTCACC \\
\hline Klf2 & TTCGGTCTCTTCGACGACG & TGCGAACTCTTGGTGTAGGTC \\
\hline Lamp1 & CAGATGTGTTAGTGGCACCCA & TTGGAAAGGTACGCCTGGATG \\
\hline Mitf & GCCTCCAAGCCTCCGATAAG & GCACTCTCTGTTGCATGAACT \\
\hline
\end{tabular}

\section{Continue on next page}


Table 4: Continued from previous page

\begin{tabular}{|c|c|c|}
\hline Primer & Sequence $\left(5^{\prime} \rightarrow 3^{\prime}\right)$-Forward & Sequence $\left(5^{\prime} \rightarrow 3^{\prime}\right)$-Reverse \\
\hline Nd1 & CAAAGGCCCCAACGTTGTAGG & AGGGTGATGGTAGATGTGGCG \\
\hline Nd6 & AGGATTGGTGCTGTGGGTGA & АСССТGАССССТСТССТTСАТ \\
\hline Ndufs3 & TGTGGCTGAAATCTTGCCCAA & AGTTGAAGCGCAGAGACAACA \\
\hline Npc1 & GCACCTTTTACCATCACTCCTG & GGCCACAGACAATAGAGCAGT \\
\hline Npc2 & CAAAGGACAGTCTTACAGCGT & GGATAGGGCAGTTAATTCCACTC \\
\hline Nrf1 & AGGAACACGGAGTGACCCAA & TATGCTCGGTGTAAGTAGCCA \\
\hline Nrf2 & TTAAACCTGCGGACACTGTTG & GTATCCCAAGGCGTTCTTGTT \\
\hline Polg & GCTGCACGAGCAAATCTTCG & GTCCAGGTTGTCCCCGTAGA \\
\hline Polrmt & GGACTCCCCGGCAAAGAAG & CGCCACATCCACCCTGTTC \\
\hline S1pr1 & TTCCACCGACCCATGTACTAT & GCGAGGAGACTGAACACGG \\
\hline S1pr2 & CATCGTCATCCTCTGTTGCG & GCCTGCCAGTAGATCGGAG \\
\hline S1pr3 & CGGCATCGCTTACAAGGTCAA & GCCACGAACATACTGCCCT \\
\hline Sdha & CAGCATGTGTTACCAAGCTGT & GGTGTCGTAGAAATGCCACCT \\
\hline Sdhd & САTCTCTCCACTGGACTAGCG & TCCATCGCAGAGCAAGGATTC \\
\hline Sgpl1 & CCTAGCACAGACCTTCTGATGT & ACTCCATGCAATTAGCTGCCA \\
\hline Sgpp1 & GCAACTGGCCGCTCTACTG & AGAAGACCTCCAACTTGACCA \\
\hline Sphk1 & GCTCTGGTGGTCATGTCTGG & CACAGCAATAGCGTGCAGT \\
\hline Sphk2 & ATGGCATCGTCACGGTCTC & CTCCCAGTCAGGGCGATCTA \\
\hline Tfam & ATGGCGTTTCTCCGAAGCAT & TCCGCCCTATAAGCATCTTGA \\
\hline Tfb2m & CCAAGGAAGGCGTCTAAGGC & CTTTCGAGCGCAACCACTTTG \\
\hline Tfeb & ACCTGTCCGAGACCTATGGG & CGTCCAGACGCATAATGTTGTC \\
\hline Tpp1 & CCTCCACACGGTGCAAAAATG & CTCTGCTTGTCGGATGCTCAG \\
\hline Uqcrc1 & GGGGCACAAGTGCTATTGC & GTTGTCCAGCAGGCTAACC \\
\hline Uqcrfs1 & CTGAATACCGCCGCCTTGAA & ATGCGACACCCACAGTAGTTA \\
\hline mtDNA & CGAAAGGACAAGAGAAATAAGG & CTGTAAAGTTTTAAGTTTTATGCG \\
\hline B-globin & СААСТTСАТССАСGTTСАСС & GAAGAGCCAAGGACAGGTAC \\
\hline
\end{tabular}


Table 5: Mouse Primers used for qPCR in this study

\begin{tabular}{|c|c|c|}
\hline Primer & Sequence $\left(5^{\prime} \rightarrow 3^{\prime}\right)$-Forward & Sequence $\left(5^{\prime} \rightarrow 3^{\prime}\right)$-Reverse \\
\hline $12 S$ & AGACACCTTGCCTAGCCACAC & CGGTGGCTGGCACGAAATTTA \\
\hline Atg5 & ATGCGGTTGAGGCTCACTTTA & GGTTGATGGCCCAAAACTGG \\
\hline Atp6v0d1 & GCTACTTGGAGGGATTAGTGCG & GCGGAACTCTACTACCATCTTCT \\
\hline Atp6v1a & CCGTACTCCGCACTGGTAAAC & TGGGGATGTAGATACTTTGGGT \\
\hline CAD & GCAAGTGGTTTGAATCCTCGG & CATTGGGGTCCACGAATGG \\
\hline Cox5a & ATGCCTGGGAATTGCGTAAAG & TGCGAACAGCACTAGCAAAAT \\
\hline Creb & CAGTGGGCAGTACATTGCCAT & CTGCTGTCCATCAGTGGTCTG \\
\hline Ctsb & TTGCGTTCGGTGAGGACATAG & GCAGGAGCCCTGGTCTCTA \\
\hline Cxcl10 & CCAAGTGCTGCCGTCATTTTC & GGCTCGCAGGGATGATTTCAA \\
\hline Cytb & GCTTTCCACTTCATCTTACCATTTA & TGTTGGGTTGTTTGATCCTG \\
\hline Dguok & CGTCTCCGAGCGTCTTTCC & CACAGCGATGTTGCCTTCA \\
\hline Fpn & TGGAACTCTATGGAAACAGCCT & TGGCATTCTTATCCACCCAGT \\
\hline Fth1 & CAAGTGCGCCAGAACTACCA & ACAGATAGACGTAGGAGGCATAC \\
\hline Ftl1 & AGGGCGTAGGCCACTTCTT & CTGGGTTTTACCCCATTCATCTT \\
\hline Gaa & GGGCCTGCACCCTTATCTC & GGTCGGTACGTCTTCCACAG \\
\hline Gapdh & TGTGTCCGTCGTTCTGA & CCTGCTTCACCACCTTCTTGA \\
\hline Hprt & ССТССTCAGACCGCTTTTT & AACCTGGTTCATCATCGCTAA \\
\hline Ifi44 & АTGCTCCAACTGACTGCTCG & ACAGCAATGCCTCTTGTCTTT \\
\hline Ifit1 & GCCTATCGCCAAGATTTAGATGA & TTCTGGATTTAACCGGACAGC \\
\hline Ifit3 & СCTACATAAAGCACCTAGATGGC & ATGTGATAGTAGATCCAGGCGT \\
\hline Irf3 & GAGAGCCGAACGAGGTTCAG & CTTCCAGGTTGACACGTCCG \\
\hline Irf7 & GCGTACCCTGGAAGCATTTC & GCACAGCGGAAGTTGGTCT \\
\hline Irp1 & ACTCAAGATACGGACGCTTACC & GTTGCATGACATTCCAATTCAGG \\
\hline Irp2 & TTCTGCCTTACTCAATACGGGT & AGGGCACTTCAACATTGCTCT \\
\hline Isg15 & GGTGTCCGTGACTAACTCCAT & CTGTACCACTAGCATCACTGTG \\
\hline Lamp1 & GACGGTGACCAGAGCGTTC & GTGGGCACTAGGGCATCAG \\
\hline Mthfd1 & GGGAATCCTGAACGGGAAACT & TGAGTGGCTTTGATCCCAATC \\
\hline
\end{tabular}

List of Mouse primers continued on next page 
Table 5: Continued from previous page

\begin{tabular}{|c|c|c|}
\hline Primer & Sequence $\left(5^{\prime} \rightarrow 3^{\prime}\right)$-Forward & Sequence $\left(5^{\prime} \rightarrow 3^{\prime}\right)$-Reverse \\
\hline Mthfd11 & GCATGGCCTTACCCTTCAGAT & GTACGAGCTTCCCCAGATTGA \\
\hline Mthfd2 & AGTGCGAAATGAAGCCGTTG & GACTGGCGGGATTGTCACC \\
\hline Mthfd2I & ACGAAGCCGTTATCATCTCAGG & TGTTCCCGAGAGCGATCCA \\
\hline Myd88 & AGGACAAACGCCGGAACTTTT & GCCGATAGTCTGTCTGTTCTAGT \\
\hline Nd1 & AGGCCCTAACATTGTTGGTCCA & TGTGAGTGATAGGGTAGGTGCA \\
\hline $\mathrm{Nd} 6$ & ATTAAACAACCAACAAACCCAC & TTTGGTGGTTGTCTGGGTT \\
\hline Ndufs3 & TGGCAGCACGTAAGAAGGG & CTTGGGTAAGATTTCAGCCACAT \\
\hline Nrf1 & CCCCCGAGGACACTTCTTATGATG & GGCCGTTTCCGTTTCTTCCCTGTT \\
\hline Nrf2 & AGAAATTGAGATCGACGGGACT & GCTGGGGTATAGGTTTGTTCCA \\
\hline Polg & GAGCCTGCCTTACTTGGAGG & GGCTGCACCAGGAATACCAG \\
\hline Polrmt & AGAAGGCTCCAGTAATGTCCA & CCTGCATCAGTATGCTCACAA \\
\hline Pparg & GGAAGACCACTCGCATTCCTT & GTAATCAGCAACCATTGGGTCA \\
\hline Rrm2b & GAGCCACTCCTAAGAAAGAGTTC & CAGGGAGGTCCTTTGACAAGT \\
\hline Samhd1 & CTGAGTCAGTCTCGGATTGATCT & AGCTGCTTGATATAGCGAAGTC \\
\hline Slc11a2 & TTTAGCTTTCGTAAACTCTGGGC & GTTTCCTGGGTCTAGGTAGGC \\
\hline Stat1 & TCACAGTGGTTCGAGCTTCAG & CGAGACATCATAGGCAGCGTG \\
\hline Stat2 & GTTACACCAGGTCTACTCACAGA & TGGTCTTCAATCCAGGTAGCC \\
\hline Steap3 & CTCAGCGAGGGAGGTAGAAG & TCGGATGAAGTTGTAGGTGTAGA \\
\hline Sting & TCGCACGAACTTGGACTACTG & CCAACTGAGGTATATGTCAGCAG \\
\hline Tfam & GCTCTACACGCCCCTGGTTTCTGG & TCGCTGTAGTGCCTGCTGCTCCTG \\
\hline Tfb2m & TATAGAGCCGTTGCCTGATTCT & GCCGCTTTCTTACATGCTATGTG \\
\hline Tfeb & CCACCCCAGCCATCAACAC & CAGACAGATACTCCCGAACCTT \\
\hline Tfrc & GTTTCTGCCAGCCCCTTATTAT & GCAAGGAAAGGATATGCAGCA \\
\hline Tk2 & AGCAGTGGTTTGTATTGAGGG & ACATGAGGCTCAGAGGGTTATG \\
\hline Tlr9 & ACAACTCTGACTTCGTCCACC & TCTGGGCTCAATGGTCATGTG \\
\hline Tsc2 & TGAATGCGGCCTCAACAATC & GACAGCCTTCCAAAGTGCCT \\
\hline mtDNA & ССТАТСАСССТTGССАТСАТ & GAGGCTGTTGCTTGTGTGAC \\
\hline nDNA & ATGGAAAGCCTGCCATCATG & TCCTTGTTGTTCAGCATCAC \\
\hline
\end{tabular}




\subsubsection{Cell culture devices}

The following materials and devices were used specifically for cell culture experiments carried out in this study.

Table 6: Cell culture materials and devices

\begin{tabular}{lll}
\hline \hline \multicolumn{1}{c}{ Product } & \multicolumn{1}{c}{ Supplier } & Catalog number \\
\hline $60 \times 20 \mathrm{~mm}$ TC dish & CytoOne & CC7682-3354 \\
$100 \times 20 \mathrm{~mm}$ TC dish & CytoOne & CC7682-3394 \\
$150 \times 20 \mathrm{~mm}$ TC dish & CytoOne & CC7682-3617 \\
6 -well TC plate & CytoOne & CC7682-7506 \\
12-well TC plate & CytoOne & CC7682-7512 \\
$96-$ well TC plate & CytoOne & CC7682-7596 \\
96-well TC plate black & Thermo Scientific & 137104 \\
Cell scraper & StarLab & CC7600-0202 \\
Countess cell counting chamber slides & Invitrogen & C10283 \\
Coverslips & Marienfeld & 017580 \\
Cryogenic vial 2 mL & Fisher Brand & 1050026 \\
Filter syringe $0.22 \varnothing$ Rotilabo CME & Carl Roth & SE2M35I07 \\
Syringe Inject & Becton. Dickinson and & 4606205 \\
\hline \hline
\end{tabular}




\subsubsection{Plasmids}

Transfections were carried out with pcDNA, shRNA, and siRNA listed below (Table 7).

Table 7: Plasmids used in this study

\begin{tabular}{|c|c|c|}
\hline Plasmid & Supplier & Catalog number \\
\hline PLP1, PLP2, PLP-VSVG & \multicolumn{2}{|l|}{ Kind gift from Prof. D Katschinski } \\
\hline pLKO.1-blast-scrambled & Addgene & 26701 \\
\hline Scrambled negative control & Integrated DNA Technologies & $51-01-19-09$ \\
\hline shRNA-human GAA & GE Dharmacon & RHS3979-9616754 \\
\hline siRNA-human ETV1 & Integrated DNA Technologies & 72688375 \\
\hline siRNA-human ETV1 & Integrated DNA Technologies & 72688384 \\
\hline siRNA-human KLF2 & Integrated DNA Technologies & 72688381 \\
\hline siRNA-human KLF2 & Integrated DNA Technologies & 72688378 \\
\hline siRNA-human TFEB & Integrated DNA Technologies & 73459471 \\
\hline siRNA-human TFEB & Integrated DNA Technologies & 73459474 \\
\hline siRNA-mouse ATG5 & Integrated DNA Technologies & 69223127 \\
\hline siRNA-mouse ATG5 & Integrated DNA Technologies & 69223130 \\
\hline siRNA-mouse SLC11A2 & Integrated DNA Technologies & 72093245 \\
\hline siRNA-mouse SLC11A2 & Integrated DNA Technologies & 72093248 \\
\hline siRNA-mouse TMEM173 & Integrated DNA Technologies & 70933800 \\
\hline siRNA-mouse TMEM173 & Integrated DNA Technologies & 70933803 \\
\hline siRNA-mouse TSC2 & Integrated DNA Technologies & 71074023 \\
\hline siRNA-mouse TSC2 & Integrated DNA Technologies & 71074026 \\
\hline siRNA-mouse TSC2 & Integrated DNA Technologies & 71074029 \\
\hline
\end{tabular}




\subsubsection{Antibodies}

Different primary and secondary antibodies were used for Immunoblotting (IB) and immunocytochemistry $(\mathrm{IHC})$ experiments in this thesis. These antibodies are listed below.

Table 8: Primary antibodies for immunoblotting and immunocytochemistry

\begin{tabular}{|c|c|c|c|c|}
\hline Antibody & Supplier & Catalog number & Application & Dilution \\
\hline ACC & Cell signaling & 3676 & IB & $1: 1000$ \\
\hline ACC $p S e r^{79}$ & Cell signaling & 3661 & IB & $1: 1000$ \\
\hline AKT pan & Cell signaling & 4691 & IB & $1: 1000$ \\
\hline AKT pSer 473 & Cell signaling & 4060 & IB & $1: 1000$ \\
\hline ATG5 & Cell signaling & 12994 & IB & $1: 1000$ \\
\hline CAD & Cell signaling & 9392 & IB & $1: 1000$ \\
\hline CAD pSer ${ }^{1859}$ & Cell signaling & 1266 & IB & $1: 1000$ \\
\hline DGUOK & Sigma Aldrich & HPA034766 & IB & $1: 1000$ \\
\hline ERK & Cell signaling & 4695 & IB & $1: 1000$ \\
\hline ERK pThr 204 & Cell signaling & 4376 & IB & $1: 1000$ \\
\hline ETV1 & Abcam & ab184120 & IB & $1: 1000$ \\
\hline MTHFD1 & Sigma Aldrich & HPA000704 & IB & $1: 1000$ \\
\hline MTHFD2 & Abcam & $a b 151447$ & IB & $1: 1000$ \\
\hline P70S6K1 & Cell signaling & 2708 & IB & $1: 1000$ \\
\hline P70S6K1 pThr ${ }^{389}$ & Cell signaling & 9234 & IB & $1: 1000$ \\
\hline SAMHD1 & Abcam & ab128107 & IB & $1: 1000$ \\
\hline SAMHD1 pThr ${ }^{592}$ & Cell signaling & 15038 & IB & $1: 1000$ \\
\hline SQSTM1 & Abcam & $a b 110252$ & IB & $1: 8000$ \\
\hline TSC2 & Cell signaling & 4308 & IB & $1: 1000$ \\
\hline
\end{tabular}


Table 8: Continued from previous page

\begin{tabular}{lllll}
\hline Antibody & Supplier & Catalog number & Application & Dilution \\
\hline FTH1 & Cell signaling & 3998 & IB & $1: 2000$ \\
FTL1 & Abcam & ab69090 & IB & $1: 2000$ \\
GAPDH & Sigma Aldrich & G9545 & IB & $1: 10000$ \\
GFAP & Kind gift from Prof. K-A Nave & IB/ IHC & $1: 1000$ \\
HPRT & Abcam & ab10479 & IB & $1: 4000$ \\
KLF2 & Abcam & ab203591 & IB & $1: 1000$ \\
LAMP1 & Abcam & ab24170 & IB & $1: 2000$ \\
LC3B (D11) & Cell Signaling & 3868 & IB & $1: 2000$ \\
MBP & Kind gift from Prof. K-A Nave & IB/ IHC & $1: 1000$ \\
NDUFS3 & Invitrogen & 459130 & IB & $1: 1000$ \\
NRF1 & Abcam & ab175932 & IB & $1: 1000$ \\
NRF2 & ProteinTech & $21542-1-A P$ & IB & $1: 1000$ \\
OXPHOS & Abcam & ab110413 & IB & $1: 2000$ \\
PLP & Kind gift from Prof. K-A Nave & IB/ IHC & $1: 1000$ \\
S1PR1 & Abcam & ab125074 & IB & $1: 5000$ \\
S6 & Cell signaling & 2217 & IB & $1: 2000$ \\
S6 pSer $235 / 236$ & Cell signaling & 4858 & IB & $1: 2000$ \\
TFAM & Abcam & ab138351 & IB & $1: 1000$ \\
TFEB & Novus & NBP1-67872 & IB & $1: 1000$ \\
TFRC & Abcam & ab84036 & IB & $1: 1000$ \\
UQCRC1 & ab110252 & IB & $1: 1000$ \\
\hline \hline
\end{tabular}


Table 9: Secondary antibodies for immunoblotting and immunocytochemistry

\begin{tabular}{llll}
\hline \hline \multicolumn{1}{c}{ Antibody } & \multicolumn{1}{c}{ Supplier } & \multicolumn{1}{c}{ Code } & \multicolumn{1}{c}{ Applications } \\
\hline Goat anti-mouse IgG & Dianova & $115-035-146$ & IB \\
Goat anti-mouse Alexa 488 & Life Technologies & A-11001 & IHC \\
Goat anti-rabbit lgG & Dianova & $115-035-144$ & IB \\
\hline \hline
\end{tabular}

\subsubsection{Cell lines}

HeLa and DU145 cells used in this work were purchased from ATCC. In addition, HEK293T cells, obtained from Prof. Katschinki's Lab, were used as lentiviral packaging cells for the generation of stable GAA knock-downs. Niemann-Pick Type A/B patient and age-matched control fibroblasts were obtained from the University of Goettingen Medical Center (Prof. Robert Steinfeld) and maintained under the UMG's ethical guidelines.

\subsubsection{Instruments}

All instruments and equipment used in this study are listed in Table 10 below.

Table 10: List of instruments and equipment

\begin{tabular}{ll}
\hline \hline \multicolumn{1}{c}{ Instrument } & \multicolumn{1}{c}{ Manufacturer } \\
\hline Ace Block Digital Dry Bath & Labnet \\
Amaxa 4D-Nucleofector & Lonza \\
Bio-Rad Power Pack HC Mini-Protean Tetra System & Bio-Rad \\
CAWOMAT 2000 & CAWO \\
Centrifuge 5415R & Eppendorf \\
Centrifuge 5810R & Eppendorf \\
Centrifuge Allegra X-15R & Beckman Coulter \\
\hline
\end{tabular}


Table 10: Continued from previous page

\begin{tabular}{ll}
\hline Instrument & Manufacturer \\
\hline Countess C10281 & Invitrogen \\
Cryogrider tool kit 230V & OPS Diagnostics \\
FACS Canto ${ }^{\text {TMII }}$ & BD Biosciences \\
Gene Quant 1300 & GE Healthcare \\
Hood Herasafe & Thermo Scientific \\
Incubator Heracell 150i & Thermo Scientific \\
Leica CM 1850 Cryostat & Leica Biosystems \\
Microscope Zeiss Axio Vert A1 & Zeiss \\
Multichannel pipette & Eppendorf Research \\
Nanodrop 2000C & Peqlab \\
Pipettes & Eppendorf Research \\
Pipette gun accu-jet pro & BrandTech Scientific Inc. \\
pH meter pH7110 & WTW Inolab \\
Potter S (Dounce homogenizer) & Sartorius \\
Precision balance Explorer & OHAUS \\
Quant Studio 6 Flex & Life Technologies \\
Revolver wheel & Labnet \\
Scanner Epson Perfection V850 Pro & Epson \\
Seahorse XF96 Extracellular Flux Analyzer & Agilent \\
SE600 Ruby system & GE Healthcare \\
Spinning disk confocal microscope & Perkin Elmer \\
\hline & \\
\hline
\end{tabular}


Table 10: Continued from previous page

\begin{tabular}{ll}
\hline Instrument & Manufacturer \\
\hline SYNERGYM1 microplate reader & BioTek \\
Thermocycler UNO II & Biometra \\
Vortex RS-VA10 & Phoenix Instrument \\
LSM 880 Airyscan & Zeiss \\
\hline \hline
\end{tabular}

\subsubsection{Software}

Several software programs were used to analyze most of the data obtained from experiments carried out in this study. The table below lists these software packages and their manufacturers.

Table 11: List of software programs

\begin{tabular}{llll}
\hline \multicolumn{1}{c}{ Software } & \multicolumn{1}{c}{ Manufacturer } & \multicolumn{1}{c}{ City/State } & Country \\
\hline Adobe Illustrator CS6 & Adobe Systems Inc. & San Jose, CA & USA \\
FACS DIVA ${ }^{\text {TM }}$ software & BD Biosciences & Heidelberg & Germany \\
FlowJo & FlowJo LLC & Ashland, OR & USA \\
GraphPad Prism 6 \& 7 & GraphPad Software Inc. & La Jolla, CA & USA \\
GeneSpring 13.0 & Agilent & Santa Clara, CA & USA \\
Genomatix Suite & Genomatix GmbH & Munich & Germany \\
ImageJ & NIH & Bethesda, MD & USA \\
IPA Ingenuity & Qiagen & Redwood City, CA & USA \\
Mendeley & Mendeley Ltd & London & UK \\
MetaboAnalyst 3.0 & McGill University & Montreal & Canada \\
Microsoft Office & Microsoft Corporation & Redmond, WA & USA \\
\hline \hline
\end{tabular}




\subsection{Methods}

\subsubsection{Cell culture}

\subsubsection{Fibroblast culture growth conditions}

All fibroblasts used in this study were cultured in sterile Dulbecco's Modified Eagle Medium high glucose medium (DMEM) supplemented with 10\% fetal bovine serum (FBS) and $1 \%$ Penicillin/Streptomycin (P/S), hereafter referred to as growth medium, in a humidified incubator at $37^{\circ} \mathrm{C}$ and $5 \% \mathrm{CO}_{2}$ unless otherwise noted. In all experiments, cells were harvested at $70-80$ $\%$ confluence except for mtDNA copy number experiments, in which case cells were always confluent prior to harvesting. Cells were usually seeded at least 24 hours before experiments at the following densities:

Table 12: Seeding density and application for cell culture experiments

\begin{tabular}{lll}
\hline \hline Type of cell culture plate & Seeding density & Application \\
\hline $15 \mathrm{~cm}$ plate & $2.5 \times 10^{6}$ cells & Cell lysates, nucleic acid isolation \\
$6 \mathrm{~cm}$ plate & $2.0 \times 10^{5}$ cells & Flow cytometry \\
6-well plate & $1.2 \times 10^{5} \mathrm{cells}$ & Flow cytometry \\
12-well plate & $3.5 \times 10^{4}$ cells & Microscopy \\
96-well plate & $1.2 \times 10^{4}$ cells & Microplate reader assays, respirometry \\
\hline \hline
\end{tabular}

\subsubsection{Drug and chemical treatment of adherent cells}

For each application, human dermal fibroblasts or mouse embryonic fibroblasts were seeded in the appropriate plate and at the corresponding density at least 24 hours prior to chemical treatments. The following chemicals and their concentrations (unless stated otherwise) were used for cell treatments: $50 \mu \mathrm{M}$ Chloroquine, $1 \mu \mathrm{M}$ Oligomycin, $2 \mu \mathrm{M}$ Carbonyl cyanide 3fluorophenylhydrazone (FCCP), $1 \mu \mathrm{M}$ Rotenone, $1 \mu \mathrm{M}$ Antimycin, 40 $\mu \mathrm{M}$ Desipramine, $5 \mu \mathrm{M}$

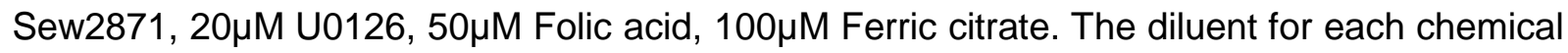
was used as the corresponding control for treatments. Unless otherwise noted, cells were treated with these drugs for 72 hours by briefly washing cells in warm PBS and replacing the growth medium with growth medium supplemented with the corresponding drug or its diluent as a control. 


\subsubsection{Generation of stable GAA knockdown cell lines}

Lentiviral suspensions were obtained by lipofectamine-mediated transfection of HEK293T packaging cells with an optimized mix of packaging plasmids (PLP1, PLP2, and PLP/VSVG) and either of GAA shRNAs targeting five different regions of GAA coding gene or scrambled non-targeting negative control shRNA. Following manufacturer's instructions, lentiviral vectors were concentrated using the Lenti-X concentrator. HeLa and DU145 cell lines were transduced with concentrated viral suspension supplemented with $8 \mathrm{ug} / \mathrm{ml}$ Polybrene and cells with puromycin resistance were selected and polyclonally expanded. Knockdown efficiency was confirmed by Western blot and qPCR. The best two knockdowns were selected for subsequent experiments.

\subsubsection{Transfection of dermal and mouse embryonic fibroblasts}

Control and patient dermal fibroblasts or MEFs were transfected with siRNAs for target genes or scrambled negative control using electroporation, following manufacturer's instructions. Briefly, after collecting cells as described in section 2.2.1.5, the cell pellet was resuspended in warm growth medium and $20 \mu \mathrm{L}$ of the suspension was applied to an improved Neubauer cell counting chamber. The cell density was determined by the formula:

$N \times 10^{4}$ where $N$ is the average cell number in the four corner squares

Following cell density determinations, $1.5 \times 10^{6}$ cells were pelleted and the excess medium was aspirated. $100 \mu \mathrm{L}$ of Amaxa transfection working reagent (made of $263 \mu \mathrm{L}$ of buffer and $62.5 \mu \mathrm{L}$ of supplement) was added to the cell pellet. siRNA vectors were added to a final concentration of $10 \mathrm{nM}$ and the cell pellet was resuspended by gently pipetting up and down. The cell suspension was transferred to labeled electroporation cuvettes and electroporated with the appropriate program for dermal fibroblasts or MEFs using the Amaxa 4D Nucleofector. The electroporated cells were then incubated at RT for 10 minutes, resuspended in warm growth medium and passed into appropriate plates depending on further applications. The transfected cells were cultured in normal growth conditions and harvested after 72 hours. 


\subsubsection{Harvesting of adherent fibroblasts}

For the preparation of whole cell lysates, nucleic acid isolation or cell iron measurements, adherent cells were harvested by aspirating the growth medium and washing once in warm PBS. Growth medium is then replaced with $10 \mathrm{~mL}$ ice-cold PBS and the cells are scraped on ice. The cell suspension is then collected and distributed into appropriate tubes for cell lysate preparation and nucleic acid isolation. The cell suspension is then pelleted at $2500 \mathrm{rpm}, 4^{\circ} \mathrm{C}$ for 5 minutes. The supernatant is discarded and the pellet is used for the required application or is snap frozen in liquid nitrogen and stored at $-80^{\circ} \mathrm{C}$ for later use. For all other determinations including flow cytometry, cryopreservation and transfection of fibroblasts adherent cells were collected by aspirating the growth medium, washing in warm PBS and detaching cells with TrypLE Express enzyme during 5 minutes incubation at $37^{\circ} \mathrm{C}$. Trypsin activity is blocked by adding twice the volume of growth medium. The cell suspension is then collected into labeled falcon tubes and centrifuged at centrifuged at $1500 \mathrm{rpm}, 4^{\circ} \mathrm{C}$ for 5 minutes. The supernatant is discarded and the cell pellet is used for subsequent applications.

\subsubsection{Cryopreservation and thawing of fibroblasts}

To freeze fibroblasts, cells were collected by trypsinization as described before and the cell pellet was resuspended in freezing medium. $1 \mathrm{~mL}$ aliquots of the cell suspension were made into labeled cryotubes and transferred to Mr. Frosty freezing containers filled with isopropanol. The cells were frozen at $-80^{\circ} \mathrm{C}$ overnight and then transferred to labeled cryo-boxes in a liquid nitrogen tank. On the other hand, to defrost cells, frozen cells were quickly thawed in a water bath set at $37^{\circ} \mathrm{C}$ and cultured with a suitable amount of warm growth medium at normal growth conditions. Following overnight incubation, the growth medium was aspirated, cells were washed with warm PBS and cultured in fresh warm growth medium. 


\subsubsection{Transgenic animal handling}

\subsubsection{Mouse lines and genotyping}

All mouse lines used in this study were bred and housed under standard conditions at the animal facility of the European Neuroscience Institute with access to food and water ad libitum. All animal experiments were carried out in accordance with the European guidelines for animal welfare and were approved by the Lower Saxony Landesamt fur Verbraucherschutz and Lebensmittelsicherheit (LAVES), registration number 15-1883. Mouse lines used for experiments in this study included GAA ${ }^{-/-}$mice (Jackson's lab, USA) and their wild-type littermate controls.

\subsection{Isolation of genomic DNA from mouse tails}

Genomic DNA (gDNA) was extracted by digesting $1 \mathrm{~cm}$ of mouse tails in $300 \mu \mathrm{L}$ SNET buffer supplemented with $50 \mu \mathrm{g} / \mathrm{ml}$ proteinase $\mathrm{K}$ at $55^{\circ} \mathrm{C}$ for 2 hours in a thermomixer. The lysed samples were centrifuged at $11000 \times \mathrm{g}$ for 20 minutes and the supernatant was collected. $750 \mu \mathrm{L}$ of absolute ethanol was added to the supernatant and inverted 4-6 times to completely precipitate gDNA. The precipitated gDNA was pelleted at $11000 \times \mathrm{g}$ for 30 minutes, washed with $900 \mu \mathrm{L}$ of $70 \%$ ethanol and centrifuged again at $11000 \times \mathrm{g}$ for 20 minutes. The supernatant was removed and the isolated gDNA was air dried and resuspended by adding $50 \mu \mathrm{L}$ TE buffer and placing the samples in a thermomixer at $55^{\circ} \mathrm{C}$ for 30 minutes with vortexing. The concentration of DNA was measured using the Nanodrop and the gDNA was used for genotyping or stored at $-20^{\circ} \mathrm{C}$.

\subsection{Genotyping of GAA mice}

Following gDNA isolation from mouse tails, for the purpose of genotyping, gDNA was amplified using the GoTaq Flexi DNA polymerase kit according to manufacturer's protocol. Briefly, each $25 \mu \mathrm{L}$ reaction was made of $5 \mu \mathrm{L}$ of $5 X$ reaction buffer, $2 \mu \mathrm{L}$ of $25 \mathrm{mM} \mathrm{MgCl}_{2}, 0.5 \mu \mathrm{L}$ of $10 \mathrm{mM}$ dNTPs, $0.5 \mu \mathrm{L}$ each of $10 \mu \mathrm{M}$ primers, $0.2 \mu \mathrm{L}$ of $5 \mathrm{U} / \mu \mathrm{L}$ Taq polymerase, $12.8 \mu \mathrm{L}$ of nuclease-free water and $3 \mu \mathrm{L}$ of gDNA template. The following thermal cycling protocol was used for gDNA amplification: initial denaturation at $95^{\circ} \mathrm{C}$ for 10 minutes, 33 cycles of denaturation at $95^{\circ} \mathrm{C}$ for 30 seconds, annealing at $58^{\circ} \mathrm{C}$ for 45 seconds and extension at $72^{\circ} \mathrm{C} 1$ minute, followed by 7 minutes of final extension at $72^{\circ} \mathrm{C}$. The reaction was then held at $10^{\circ} \mathrm{C}$ indefinitely. $18 \mu \mathrm{L}$ of amplicons, mixed with $5 \mathrm{X}$ loading buffer along with a standard DNA marker were 
electrophoresed in 1.5\% agarose gels. The gels were previously poured by dissolving $1.5 \mathrm{~g}$ of agarose in $100 \mathrm{~mL}$ of $1 \mathrm{X}$ TAE buffer, boiled in a microwave to dissolve the agarose, allowed to cool to about $60^{\circ} \mathrm{C}$ and mixed with $3 \mu \mathrm{L}$ of $10 \mathrm{mg} / \mathrm{mL}$ ethidium bromide and poured into a horizontal chamber. 1X TAE buffer was used as running buffer for the electrophoresis, which was run at a steady voltage of $120 \mathrm{~V}$. Following electrophoresis, the DNA was imaged with a UV gel documentation system and the size of DNA fragments was determined by comparing bands to the DNA marker.

Table 13: Primers used for GAA mouse genotyping

\begin{tabular}{ll}
\hline \hline Primer & Sequence \\
\hline IMR7067 & TCC TGA GCC CAA ACA CTT CT \\
IMR7077 & ATT GTT GCA CAA CGC TCT TG \\
IMR7297 & CGT TGG CTA CCC GTG ATA TT \\
\hline \hline
\end{tabular}

\subsubsection{Harvesting of mouse tissues}

For biochemical and molecular biology applications, mouse tissues were harvested, snap frozen in liquid nitrogen and stored at $-80^{\circ} \mathrm{C}$ until usage. Briefly, GAA ${ }^{-/}$mice and their wild-type littermates were anesthetized with isoflurane following which they were euthanized by cervical dislocation. Each mouse was decapitated and the head was sprayed with $70 \%$ Ethanol. The skull was opened after peeling off the skin of the scalp and the brain was removed and placed in a dish containing PBS. With the help of a light microscope, the brain was dissected into cortices, hippocampi, and rest of the brain. These were transferred into Eppendorf tubes and snap frozen in liquid nitrogen. For other tissues, the rest of the body of the mouse was placed in a dish and sprayed with $70 \%$ Ethanol. The abdomen was opened and a small piece of the liver was cut into an Eppendorf tube. The diaphragm was cut open and the heart was also removed, rinsed in PBS and placed in an Eppendorf tube. Quadriceps were also obtained from the mice after peeling off the thigh skin and placed in an Eppendorf tube. Similarly, these samples were also snap frozen in liquid nitrogen. All samples were then stored at $-80^{\circ} \mathrm{C}$. Prior to usage for other applications, tissues were powdered by grinding under liquid nitrogen using a cryogrinder toolkit. Aliquots of powdered tissue were then made and used immediately or stored at $-80^{\circ} \mathrm{C}$. 


\subsubsection{Perfusion and fixation of mouse tissues and immunohistochemistry}

Immunohistochemical assays provide reliable techniques to assess the localization and/or distribution of cellular components in tissues. The success of these approaches depends largely on adequate sample preparation, where cellular components of samples are preserved as closely as possible to their native states. To this end, mice were anesthetized with $40 \mathrm{mg} / \mathrm{mL}$ chloral hydrate in PBS by abdominal peritoneal injection. Mice were then fixed to a surgical platform and the abdomen was cut open along both sides until the diaphragm, which was then cut to expose the beating heart. Cardiac perfusion was then carried out first with cold $100 \mathrm{U} / \mathrm{mL}$ heparin in PBS followed by cold 4\% Paraformaldehyde (PFA) in PBS. The whole brain was carefully harvested as described before (see previous section) and post-fixed in $5 \mathrm{~mL}$ of $4 \%$ PFA in PBS overnight at $4^{\circ} \mathrm{C}$. After slow dehydration in $12 \%, 15 \%$, and $18 \%$ sucrose gradient solutions, the brain was embedded in OCT (Tissue Tek) in a cryomold, frozen in isopentane set in liquid nitrogen and stored at $-80^{\circ} \mathrm{C}$. For immunohistochemistry $(\mathrm{IHC}), 10 \mu \mathrm{M}$ thick sagittal sections of the cortex were cut with a cryostat and placed on microscope glass slides. Brain sections were then washed twice in PBS for a minute, incubated with $50 \mathrm{mM} \mathrm{NH}_{4} \mathrm{Cl}$ in PBS for 10 minutes and rinsed with PBS. Sections were blocked and permeabilized in IHC blocking buffer for 1 hour at RT, following which they were labeled with diluted primary antibodies in IHC blocking buffer by overnight incubation at $4^{\circ} \mathrm{C}$. The sections were rinsed multiple times in PBS, washed for a further three times in PBS for 10 minutes and incubated in diluted fluorescently labeled secondary antibodies at RT for 1-2 hours. Sections were rinsed and washed once in PBS for 10 minutes, counterstained with DAPI (1:5000 in PBS) for 5 minutes at RT in the dark, washed once in PBS for 15 minutes (protected from light) and mounted with Mowiol@ 4-88. Sections were left to dry overnight at RT in the dark and sealed. Slides were immediately imaged or stored in slide boxes at $4^{\circ} \mathrm{C}$.

\subsubsection{Isolation of mitochondria from mouse tissue}

Fresh mouse cortices were harvested as described in section 2.2.2.2, washed twice in $3 \mathrm{~mL}$ cold PBS and cut into smaller pieces. The pieces of tissue were transferred into a pre-chilled Dounce homogenizer on ice and $1 \mathrm{~mL}$ of Homogenization buffer $A$ was added to it. Dounce homogenization was performed by potting tissues on ice for 50 strokes. Samples were transferred into $1.5 \mathrm{~mL}$ Eppendorf tubes and spun at $700 \mathrm{xg}$ for 10 minutes at $4^{\circ} \mathrm{C}$. The supernatant was transferred into new Eppendorf tubes. The pellet was resuspended in $1 \mathrm{~mL}$ Homogenization buffer A and placed in the Dounce homogenizer. Homogenization was 
performed again as before. The samples were collected into Eppendorf tubes and centrifuged again at $700 \mathrm{xg}$ for 10 minutes at $4^{\circ} \mathrm{C}$. The supernatant was collected, pooled with previously collected supernatant and centrifuged for a further 10 minutes at $700 \times \mathrm{g}, 4^{\circ} \mathrm{C}$. The supernatant was again collected and further centrifuged at $11000 \mathrm{xg}$ for 15 minutes at $4^{\circ} \mathrm{C}$. The supernatant was collected into new Eppendorf tubes as the cytosolic fraction. The mitochondrial pellet was washed with $500 \mu \mathrm{L}$ of Homogenization buffer B and centrifuged again at $11000 \times \mathrm{g}$ for 5 minutes at $4^{\circ} \mathrm{C}$. The supernatant was discarded and the isolated mitochondria (pellet) were resuspended in 200uL of Homogenization buffer $\mathrm{B}$. The mitochondrial protein concentration was determined using the Pierce BCA assay (described later see section 2.2.3.4). Purified mitochondria were used immediately for subsequent applications or aliquoted, snap frozen in liquid nitrogen and stored at $-80^{\circ} \mathrm{C}$.

\subsubsection{Biochemical procedures}

\subsubsection{Preparation of whole cell lysates from adherent cells}

Whole cell lysates were prepared by resuspending previously harvested adherent cell pellets on ice in $50-150 \mu \mathrm{L}$ of lysis buffer (1.5\% N-dodecyl $\beta$-D-maltoside in PBS supplemented with protease and phosphatase inhibitor), depending on pellet size. The cell suspension was transferred to $1.5 \mathrm{ml}$ Eppendorf tubes and lysed by rotation at $4^{\circ} \mathrm{C}$ for 30 minutes. The cell homogenate was then centrifuged at $16000 \mathrm{rpm}, 4^{\circ} \mathrm{C}$ for 20 minutes. The whole cell lysate (supernatant) was collected into new 1.5mL Eppendorf tubes and the protein concentration was determined by the Bradford Assay. In order to avoid freeze-thaw cycles, lysates were either used for further determinations or aliquoted and stored at $-80^{\circ} \mathrm{C}$.

\subsubsection{Preparation of whole tissue lysates}

For tissue lysates, previously cryoground tissue powder in $1.5 \mathrm{~mL}$ Eppendorf tubes were resuspended in $250-400 \mu \mathrm{L}$ of RIPA buffer supplemented with protease and phosphatase inhibitor (depending on tissue size), vortexed briefly and lysed by rotation at $4^{\circ} \mathrm{C}$ for 1 hour. Following lysis, the tissue homogenate was centrifuged at $16000 \mathrm{rpm}, 4^{\circ} \mathrm{C}$ for 20 minutes. The tissue lysate (supernatant) was collected into new 1.5mL Eppendorf tubes and the protein concentration was determined by the Pierce Assay. In order to avoid freeze-thaw cycles, lysates were used for further determinations or aliquoted, snap frozen in liquid nitrogen and stored at $-80^{\circ} \mathrm{C}$. 


\subsubsection{Protein concentration determination using Bradford assay}

Protein concentrations of whole cell lysates were determined using Protein Assay Dye Reagent Concentrate (Bio-Rad) following manufacturer's protocol. Along with standards using bovine serum albumin (BSA), the protein concentration of cell lysates was determined by mixing, in duplicates, $800 \mu \mathrm{L}$ of $\mathrm{dd}_{2} \mathrm{H}_{2} \mathrm{O}, 1 \mu \mathrm{L}$ of sample or standard and $200 \mu \mathrm{L}$ of protein assay dye reagent concentrate in a glass tube and vortexed briefly. The tubes containing mixtures were incubated at RT in the dark for 5 minutes and transferred into plastic cuvettes. The absorbance at 595 $\mathrm{nm}$ was measured using a GeneQuant 1300 spectrophotometer. The protein concentration of the cell lysates was calculated based on the standard calibration curve using Microsoft Excel 2013.

\subsubsection{Protein concentration determination using Pierce BCA assay}

Protein concentrations of tissue lysates and purified tissue mitochondria were determined using Pierce BCA Protein Assay Kit (Bio-Rad) according to manufacturer's instructions. Along with albumin standards, the protein concentration of tissue lysates or purified mitochondria was determined by mixing, in duplicates, $1 \mathrm{~mL}$ of Pierce $\mathrm{BCA}$ working reagent (reagents $\mathrm{A}$ and $\mathrm{B}$, $50: 1)$ and $50 \mu \mathrm{L}$ of standards or 1:40 diluted samples in a glass tube and vortexed briefly. The tubes containing mixture were incubated at $37^{\circ} \mathrm{C}$ for 30 minutes in the dark and transferred into plastic cuvettes. The absorbance at $562 \mathrm{~nm}$ was measured using a GeneQuant 1300 spectrophotometer. The protein concentration of the cell lysates was calculated based on the standard calibration curve while taking into consideration the sample dilution factor using Microsoft Excel 2013.

\subsubsection{SDS-PAGE}

SDS-PAGE is a widely used technique to separate denatured molecules according to their weight. Briefly, using the Bio-Rad Gel preparation system, $1 \mathrm{~mm}$ thick $12 \%$ resolving gels were made with the composition described previously in section 2.1.3, poured between the gel glass plates and covered with isopropanol to prevent air bubbles. After the gels were set, the excess isopropanol was drained and the gels were rinsed with ${ }_{d d} \mathrm{H}_{2} \mathrm{O}$. The excess water was also drained and the $4 \%$ stacking gel (composition previously described) was poured on top of the polymerized resolving gel. A desired multi-well comb was quickly inserted into the stacking gel and allowed to set. The polymerized gel was used immediately or stored in a container filled 
with $1 \mathrm{X}$ running buffer at $4^{\circ} \mathrm{C}$. To run the SDS-PAGE, the Mini-Protean Tetra System (Bio-Rad) was set up with gels without the combs and filled with $1 \mathrm{X}$ running buffer. An equal amount of protein from cell or tissue lysates was mixed with $4 \mu \mathrm{L}$ of $6 \mathrm{X}$ SDS loading buffer, made up to $24 \mu \mathrm{L}$ with lysis buffer, boiled at $95^{\circ} \mathrm{C}$ for 5 minutes and loaded per well in a 10 -well gel along with a molecular weight standard, the PageRuler plus Prestained ladder. For 15-well gels, an equal amount of protein and $3 \mu \mathrm{L}$ of $6 \mathrm{X}$ SDS made up to $18 \mu \mathrm{L}$ with lysis buffer instead was loaded per well after boiling at $95^{\circ} \mathrm{C}$ for 5 minutes. Within the stacking gel, the electrophoresis was run at an initial constant voltage of $80 \mathrm{~V}$ and switched to constant $180 \mathrm{~V}$ when samples were in the resolving gel and run until samples reached the bottom of the gel.

\subsubsection{Immunoblotting}

Following SDS-PAGE and in order to detect specific target proteins, Western blot was carried according to protocols described by (Gallagher et al 2011). Briefly, polyvinylidene fluoride (PVDF) membranes were rinsed for $15 \mathrm{sec}$ in methanol to activate them and washed with $\mathrm{dd}_{2} \mathrm{H}_{2} \mathrm{O}$ for 3 minutes. Previously run gels were disassembled from the gel glass plates, stacking gels were cut off and the gels along with activated PVDF membranes were equilibrated at RT in 1X transfer buffer (contains 20\% methanol and cold at all times). The transfer sponges and sheets of Whatman paper were also soaked in $1 \mathrm{X}$ transfer buffer. Using the tank transfer system, the transfer sandwich was assembled on a plastic transfer cassette completely immersed in $1 \mathrm{X}$ transfer buffer to avoid trapping air bubbles. The order of the transfer assembly was as follows: place a soaked sponge on the bottom half of the plastic transfer cassette followed by two sheets of wet Whatman paper, place gel on top of the papers and remove any air bubbles by gently rolling a roller over the gel surface, place wet PVDF membrane on top of the gel and roll over membrane surface to remove any air bubbles and place again two sheets of wet Whatman paper followed by one wet transfer sponge. The top half of the transfer cassette was locked in place and the transfer sandwich was placed in the transfer apparatus in a tank filled with $1 \mathrm{X}$ transfer buffer while ensuring the correct orientation (bottom half towards cathode). A cold ice pack was also placed in the tank to avoid excessive heating of the transfer buffer. The proteins were transferred from gel to PVDF membrane at constant $100 \mathrm{~V}$ for 1.5 hours in a cold room. 


\subsubsection{Immunodetection}

After complete electrotransfer of proteins onto PVDF membranes, the transfer sandwich was disassembled and the PVDF membranes were blocked in blocking buffer with mild shaking for 1 hour at RT. The membranes were then washed three times in 1X TBST for 5 minutes and labeled with primary antibodies (diluted in blocking buffer) by overnight incubation at $4{ }^{\circ} \mathrm{C}$. The membranes were washed three times in 1X TBST for 10 minutes and incubated with appropriate HRP-conjugated secondary antibodies diluted in blocking buffer at RT for 1 hour and washed three more times in 1X TBST for 10 minutes. Signals representing proteins by chemiluminescence were detected by draining excess $1 \mathrm{X}$ TBST on membranes, incubating membranes in Luminata Western HRP substrate for 3 minutes and developing signals on medical X-ray films using the AGFA Curix 60 processor. The films were scanned with the Epson Perfection V850 Pro Scanner and band densities were quantified with Image J software.

\subsubsection{BN-PAGE and respiratory chain complex activity staining}

BN-PAGE, unlike SDS-PAGE, resolves non-denatured proteins thereby also preserving their physiological interactions with other proteins. As a result, the electrophoretic mobility depends not only on the charge-to-mass ratio but also on the size and shape of proteins. As this technique was developed to separate mitochondrial proteins and complexes, under low detergent conditions, respiratory chain supercomplexes are preserved, allowing for further ingel staining of their activities (Schägger and Pfeiffer, 2000; Schägger and von Jagow, 1991). We took advantage of this technique to characterize the role of lysosomal malfunction in mitochondrial complex assembly and function.

\subsection{BN-PAGE: sample preparation}

$50 \mu \mathrm{g}$ each of previously isolated mitochondria from mouse cortices was centrifuged at 14000rpm, $2^{\circ} \mathrm{C}$ for 5 minutes. The supernatant was removed and the mitochondrial pellet was resuspended in $50 \mu \mathrm{L}$ of ice-cold digitonin solubilization buffer. The mitochondria were solubilized by pipetting up and down (10 times) without generating foam. The mitochondria were mixed on a shaker at RT for 10 seconds and put back on ice for 15 minutes to complete solubilization. To remove insoluble material, the samples were centrifuged at $14000 \mathrm{rpm}, 2^{\circ} \mathrm{C}$ for 5 minutes and the supernatant was transferred into new pre-cooled Eppendorf tubes. $5 \mu \mathrm{L}$ of $10 \mathrm{X} \mathrm{BN}-$ loading dye was added to $45 \mu \mathrm{L}$ of the solubilized mitochondria and cleared by 
spinning at $14000 \mathrm{rpm}, 2^{\circ} \mathrm{C}$ for 2 minutes. Each $50 \mu \mathrm{L}$ sample was loaded per well in a $6-16.5 \%$ gradient gel along with a molecular weight standard.

\subsection{BN-PAGE: casting gels and electrophoresis}

For native separation of mitochondrial protein complexes by BN-PAGE, the protocol described in Dekker et al. (1997) was used. The gels were cast and run using the SE600 Ruby system. Briefly, resolving gels were made from equal volumes of $6 \%$ and $16.5 \%$ acrylamide (stock: a mixture of $48 \%$ acrylamide and $1.5 \%$ bisacrylamide) in $1 \mathrm{X} \mathrm{BN}-$ gel buffer supplemented with $0.44 \%$ APS and TEMED and cast using a gradient gel maker with pump. The stacking gel contained $4 \%$ acrylamide solution in $1 \mathrm{X}$ BN-gel buffer, $0.08 \%$ APS and $0.133 \%$ TEMED. Following loading of samples in the gels, the cathode buffer was gently overlaid on the samples. The gel was then run in a cooled gel-chamber at $15 \mathrm{~mA}$ per gel at $100 \mathrm{~V}$ overnight at $4^{\circ} \mathrm{C}$. The cathode buffer was replaced with the anode buffer when the electrophoresis was half-way through the gel. The electrophoresis was completed just prior to the blue loading dye running out of the gel into the anode buffer.

\subsection{BN-PAGE: enzyme activity staining}

To assess the activity of complex IV of the respiratory chain, we took advantage of the COXcatalyzed reaction, which is coupled to the oxidation of 2, 2-diaminobenzidine (DAB). Briefly, electrophoresed gels were equilibrated in 50mM Potassium phosphate buffer $(\mathrm{pH} 7.4)$ for at least 15 minutes at RT. The gels were then incubated in $10 \mathrm{~mL}$ of complex IV activity staining buffer (see section 2.1.3) at $30^{\circ} \mathrm{C}$ until the brown bands of oxidized DAB were visible. The gels were then rinsed briefly in equilibration buffer and scanned using the Epson Perfection V850 Pro Scanner. The intensity of DAB stains was quantified using Image $\mathrm{J}$ software. 


\subsubsection{Molecular biology}

\subsubsection{RNA isolation from cells}

Ribonucleic acid (RNA) was isolated from cells using the CRYSTAL RNA Mini Kit (BioLab) according to manufacturer's protocol. Cell pellets were lysed by adding $400 \mu \mathrm{L}$ of Lysis Solution $\mathrm{RL}$ supplemented with 1\% 2-Mercaptoethanol ( $\beta$-SH), incubated at RT for 2 minutes, resuspended and incubated for a further 3 minutes at RT. The lysed samples were transferred to Spin Filter D column placed in Receiver Tubes $2 \mathrm{~mL}$ and centrifuged for 2 minutes at 12000rpm. The column was discarded and $400 \mu \mathrm{L}$ of $70 \%$ ethanol was added to the flowthrough, mixed gently by pipetting to precipitate RNA, transferred to Spin Filter R column in Receiver Tubes $2 \mathrm{~mL}$ and centrifuged again for 2 minutes at 12000rpm. The flow-through was discarded and the column was washed with $500 \mu \mathrm{L}$ of Washing Solution HS, followed by washing with $700 \mu \mathrm{L}$ of Washing Solution LS at 10000rpm centrifugation for 1 minute each. The RNA was dried by centrifuging columns in Receiver Tubes $2 \mathrm{~mL}$ at $12000 \mathrm{rpm}$ for 2 minutes. The RNA was eluted into RNAse-free $1.5 \mathrm{~mL}$ collection tubes by adding $20-50 \mu \mathrm{L}$ of nucleasefree water, incubated at RT for 1 minute and centrifuged at 8000rpm for 1 minute. The RNA concentration was determined as described (see 2.2.4.4) and the RNA was either used immediately or frozen at $-80^{\circ} \mathrm{C}$.

\subsubsection{RNA isolation from tissues}

Isolating RNA from tissues was found to be impractical with the commercial kit we used for purifying RNA from cells. As a result, isolating RNA from tissues was carried using the TRI reagent (Sigma) according to protocols described by Rio et al. (2010) with slight modifications. Cryoground tissues were homogenized in $1 \mathrm{~mL}$ of TRI reagent following 5 minutes incubation at $\mathrm{RT}$ in $1.5 \mathrm{~mL}$ Eppendorf tubes. $0.2 \mathrm{~mL}$ of chloroform was added per $1 \mathrm{~mL}$ of TRI reagent. Tubes were securely capped, shaken vigorously by hand for 15 seconds and incubated at RT for 2-3 minutes. The samples were centrifuged at $12000 \mathrm{x} \mathrm{g}$ for 15 minutes at $4^{\circ} \mathrm{C}$. The aqueous phase was removed and placed in new RNAse-free 1.5mL Eppendorf tubes. RNA was precipitated by adding $0.5 \mathrm{~mL}$ of isopropanol per $1 \mathrm{~mL}$ of TRI reagent used and incubating at $\mathrm{RT}$ for 10 minutes and pelleted by centrifugation at $12000 \mathrm{xg}$ for 10 minutes at $4^{\circ} \mathrm{C}$. The supernatant was removed and the RNA pellet was washed by adding $1 \mathrm{~mL} 75 \%$ Ethanol per $1 \mathrm{~mL}$ of TRI reagent, vortexed briefly and centrifuged at $7500 \times \mathrm{g}$ at 5 minutes at $4^{\circ} \mathrm{C}$. The supernatant was removed and the RNA pellet was air-dried at RT for 5-10 minutes, resuspended in $50 \mu \mathrm{L}$ of RNAse-free water and incubated in a heat block at $55^{\circ} \mathrm{C}$ for $10-15$ 
minutes. The RNA concentration was determined as described (see 2.2.4.4) and the RNA was either used immediately or frozen at $-80^{\circ} \mathrm{C}$.

\subsubsection{Total DNA isolation from cells and tissues}

For the purpose of mtDNA copy number determination, total DNA was isolated from cells and tissues as described in Cotney et al. (2007). Briefly, cells or tissues were lysed in $500 \mu \mathrm{L}$ of DNA extraction buffer (composition described, see Table 3) and boiled for 10 minutes at $95^{\circ} \mathrm{C}$. After cell lysis, samples were cooled to RT and $100 \mu \mathrm{g}$ of RNAse A was added to each sample and incubated at $37^{\circ} \mathrm{C}$ for 3 hours. Following degradation of RNAs, $100 \mu \mathrm{g}$ of Proteinase $\mathrm{K}$ was added to each sample and incubated at $55^{\circ} \mathrm{C}$ overnight. Samples were then heated to $95^{\circ} \mathrm{C}$ and allowed to cool to RT. Total DNA concentration was determined as described (see 2.2.4.4) and the DNA was either used immediately or frozen at $-20^{\circ} \mathrm{C}$.

\subsubsection{Determination of nucleic acid concentrations}

To determine the concentration of isolated RNA or DNA, the Nanodrop (PeqLab) spectrophotometer was used. Sample absorbances against nuclease-free water blank were also measured $260 \mathrm{~nm}$ and $280 \mathrm{~nm}$ to check the quality of nucleic acids. Briefly, $1.5 \mu \mathrm{L}$ of blank or sample was applied to the Nanodrop pedestal, the arm was closed and the sample concentration and absorbances were measured. The results of nucleic acid concentration determinations were then exported as Microsoft Excel files. The samples were either stored as described previously or used immediately.

\subsubsection{5 cDNA synthesis}

For gene expression studies, isolated RNA was reverse transcribed into cDNA using the iScript cDNA synthesis kit (BioRad) according to manufacturer's instructions. Briefly, all reaction components were thawed on ice and each reaction contained $1 \mu \mathrm{g}$ of RNA, $1 \mu \mathrm{L}$ of iScript reverse transcriptase, $4 \mu \mathrm{L}$ of $5 \mathrm{X}$ iScript reaction mix and made to a final volume of $20 \mu \mathrm{L}$ with nuclease-free water in PCR strips. The reaction mix was vortexed and spun down briefly. The reaction mix was then incubated in a thermal cycler using the following protocol: priming at $25^{\circ} \mathrm{C}$ for 5 minutes, reverse transcription at $46^{\circ} \mathrm{C}$ for 20 minutes, reverse transcriptase inactivation at $95^{\circ} \mathrm{C}$ for 1 minute and holding at $4^{\circ} \mathrm{C}$ indefinitely. The synthesized cDNA was diluted a 100 fold in nuclease-free water and stored at $-20^{\circ} \mathrm{C}$ until used for qPCR. 


\subsubsection{6 qPCR}

To measure the expression of various genes in this study, quantitative real time PCR was carried out using previously synthesized cDNA. Reactions were carried out as at least technical triplicates using the iTaq Universal Sybr Green Supermix (BioRad). Each $8 \mu$ reaction per well in a 384-well plate contained 3.6 $\mu \mathrm{l}$ of Sybr Green supermix, $0.2 \mu \mathrm{l}$ each of forward and reverse primers for each gene and $4 \mu$ l of diluted cDNA template. The plate was sealed with adhesive seal, spun briefly at 2000rpm for 1 minute and incubated in the QuantStudio ${ }^{\text {TM }} 6$ Flex RealTime PCR system (Life Technologies) using the fast thermal cycling protocol described as follows: polymerase activation at $95^{\circ} \mathrm{C}$ for $30 \mathrm{~s}$, amplification for 40 cycles with steps of denaturation at $95^{\circ} \mathrm{C}$ for 3 seconds, annealing at $56^{\circ} \mathrm{C}$ for 30 seconds and extension at $60^{\circ} \mathrm{C}$ for 30 seconds. Melt-curve analyses were carried out at steps of $85^{\circ} \mathrm{C}$ for 15 seconds, $52^{\circ} \mathrm{C}$ for 30 seconds and $95^{\circ} \mathrm{C}$ for 15 seconds. Data analyses were carried out using the QuantStudio ${ }^{\mathrm{TM}}$ Real-Time PCR Software and exported as Microsoft Excel files for further analyses using the $\triangle \triangle C T$ method.

\subsubsection{Relative mtDNA copy number determination}

Relative mtDNA copy numbers were determined using the protocol described in Cotney et al. (2007). The quantitative real time PCR for mtDNA was carried out similarly to qPCR described above (see 2.2.4.6) with some modifications. Instead of cDNA, total DNA was used for this purpose. Previously isolated total DNA was diluted to $2.0 \mathrm{ng} / \mu \mathrm{L}$ in nuclease-free $\mathrm{H}_{2} \mathrm{O}$. Reactions were carried out as at least technical quadruplicates using the iTaq Universal Sybr Green Supermix. Each $10 \mu \mathrm{l}$ reaction per well in a 384-well plate contained 5.0 $\mu \mathrm{l}$ of Sybr Green supermix, $0.25 \mu$ leach of forward and reverse primers for each gene and $4.5 \mu$ l of diluted total DNA template. The following fast thermal cycling protocol was used: hold stage and polymerase activation at $95^{\circ} \mathrm{C}$ for 10 minutes, amplification for 40 cycles with steps of denaturation at $95^{\circ} \mathrm{C}$ for 15 seconds, annealing/extension at $60^{\circ} \mathrm{C}$ for 1 minute. Melt-curve analyses were carried out with a temperature step gradient in $0.5^{\circ} \mathrm{C}$ increments from 55 to $80^{\circ} \mathrm{C}$. Fluorescence was measured at each temperature following 10 seconds incubation. Data analyses were carried out using the QuantStudio ${ }^{\mathrm{TM}}$ Real-Time PCR Software and exported as Microsoft Excel files for further analyses using the $\triangle \Delta C T$ method.

\subsubsection{Flow cytometry}

Flow cytometry is a powerful laser-based technique for characterizing intracellular and cell surface molecules from cell populations. This approach was used to measure fluorescence 
intensities of markers, which we previously labeled in cells or ligands that associated with molecules of interest in this study. Experimental conditions were carried out as at least four technical replicates.

\subsubsection{Apoptosis analyses by Annexin V and Propidium lodide staining assay}

Previously seeded cells and cells with induced apoptosis ( $4 \mu \mathrm{M}$ Staurosporine treatment for 2 hours at $37^{\circ} \mathrm{C}$ ) were harvested in culture medium as described before (see 2.2.1.5). The cells were washed twice with ice-cold PBS and centrifuged at 1500rpm for 5 minutes, resuspended in $400 \mu \mathrm{L}$ of $1 \mathrm{X}$ binding buffer and transferred to FACS tubes on ice. The cells were double dyestained in suspension by adding $5 \mu \mathrm{L}$ of Annexin V-FITC and $5 \mu \mathrm{L}$ of $50 \mu \mathrm{g} / \mathrm{mL}$ Propidium iodide, vortexed briefly and incubated in the dark at RT for 15 minutes. Along with unstained controls, single dye-stained controls, and Staurosporine positive controls, the apoptotic cell population of the samples was analyzed by flow cytometry. The results were analyzed using FlowJo v10 software.

\subsubsection{Measurement of mitochondrial superoxide levels}

Mitochondrial superoxide levels were measured by flow cytometry using the MitoSOX Red Mitochondrial superoxide indicator according to manufacturer's protocol. Briefly, $5 \mathrm{mM}$ MitoSOX indicator stock was prepared by resuspending each $50 \mu \mathrm{g}$ dye in $13 \mu \mathrm{I} \mathrm{DMSO}$. The MitoSOX working reagent was made by adding the $13 \mu \mathrm{l}$ of indicator stock to $13 \mathrm{~mL}$ of warm PBS, which contained $0.5 \mu \mathrm{M}$ glucose at all times for MitoSOX experiments to yield a final dye concentration of $5 \mu \mathrm{M}$. Previously cultured cells were washed with warm PBS and stained by adding MitoSOX working reagent to cells and incubating at $37^{\circ} \mathrm{C}$ for 15 minutes. Following staining, the excess indicator was washed off cells with warm PBS and cells were harvested by trypsinization as described before (see 2.2.1.5). The cells were washed twice with PBS, centrifuged at 1500rpm for 5 minutes and resuspended in $0.5 \mathrm{~mL}$ PBS. The cell suspension was transferred to FACS tubes and along with unstained cells, stained test samples and positive controls (treated with $100 \mu \mathrm{M} \mathrm{H}_{2} \mathrm{O}_{2}$ or $100 \mu \mathrm{M}$ Antimycin for 20 minutes at $37^{\circ} \mathrm{C}$ ) mitochondrial superoxide levels were determined by flow cytometry. The results were analyzed using FlowJo v10 software. 


\subsubsection{Microplate reader assays}

\subsubsection{Measurement of lysosomal proteolytic capacity}

Lysosomal proteolytic capacity was measured using the DQ Red BSA Dye (Molecular Probes) following manufacturer's protocol. $1 \mathrm{mg}$ of dye was resuspended in $1 \mathrm{~mL}$ of PBS and $100 \mathrm{ul}$ of the resuspended dye was added to $10 \mathrm{ml}$ of warm DMEM medium. Previously seeded cells in a transparent 96 well-plate, cultured at least overnight after seeding, were loaded with 100ul per well each of the dye-containing medium and incubated at $37^{\circ} \mathrm{C}$ for 1 hour. Cells were then washed twice with warm PBS and the medium was replaced with $100 \mu \mathrm{L} /$ well of warm EBSS medium. The kinetics of DQ Red BSA digestion were recorded at respective excitation and emission maxima of $590 \mathrm{~nm}$ and $620 \mathrm{~nm}$ in a microplate reader over a $4 \mathrm{~h}$ period. The results were exported as Microsoft Excel files for further analyses.

\subsubsection{Measurement of total cell iron concentration}

Total cellular iron concentration was measured using the Iron Assay kit (Abcam) according to manufacturer's instructions. MEFs were harvested as previously described (see 2.2.1.5) and homogenized in $1 \mathrm{~mL}$ of assay buffer using a Dounce homogenizer sitting on ice. The homogenate was collected into $1.5 \mathrm{~mL}$ Eppendorf tubes and centrifuged at 16000rpm for 10 minutes at $4^{\circ} \mathrm{C}$ to remove insoluble materials. The supernatant was collected into new $1.5 \mathrm{~mL}$ Eppendorf tubes and kept on ice always during the assay. All reagents were equilibrated to RT and serial dilutions of the standard were made in assay buffer. All standards, controls, and samples were assayed as duplicates in a transparent 96-well plate. The total iron assay's initial $205 \mu \mathrm{l}$ reaction per well contained $100 \mu \mathrm{l}$ standard, sample (at different dilutions) or assay buffer as control, $5 \mu \mathrm{l}$ iron reducer, and $100 \mu \mathrm{l}$ iron probe. For ferrous iron measurement, $5 \mu \mathrm{l}$ assay buffer was used instead of iron reducer. The reaction was mixed by pipetting and incubated at RT. Following 30 minutes of incubation, $100 \mu \mathrm{l}$ of the iron probe was added to each wellcontaining standard, control, and samples, mixed and incubated at RT for a further 60 minutes. The absorbance at $593 \mathrm{~nm}$ was measured immediately in a microplate reader. The results were exported as a Microsoft Excel file where a standard curve was made. The total, ferric and ferrous iron concentrations of the cell were calculated from the standard curve and normalized to the protein amount (determined by the Bradford assay, see 2.2.3.3). 


\subsubsection{Protein concentration determination using Pierce BCA microassay}

For all experiments carried out in 96-well plates except for iron measurements, protein concentration per well of samples was always determined for normalization purposes. To this end, immediately after an assay in a 96-well plate, the assay medium was always aspirated and the cells were lysed in $125 \mu \mathrm{L}$ of ${ }_{\mathrm{dd}} \mathrm{H} 2 \mathrm{O}$ for 1 hour with mild shaking. Following cell lysis, protein concentrations were determined using the Pierce BCA Protein Assay kit. Unlike in the tube method, $100 \mu$ l of double concentrated Pierce BCA working reagent (reagents A and B, $25: 1)$ was added to each well-containing samples and blank, mixed by pipetting and incubated at $37^{\circ} \mathrm{C}$ for 30 minutes. The absorbance of the samples was measured at $562 \mathrm{~nm}$ in a microplate reader and the protein concentration was calculated from a standard curve made from albumin standards.

\subsubsection{Real Time Respirometry}

The Seahorse XF96 Extracellular Flux Analyzer provides a robust tool for assessing cellular metabolic status. We assessed oxygen consumption and extracellular acidification rates in cultured cells as respective indicators of mitochondrial respiration and glycolytic capacity. These experiments were carried out using the $\mathrm{XF}^{\mathrm{e}} 96$ extracellular flux assay kit and following manufacturer's protocols. For a MitoStress Test, cells were seeded (12000 cells/well) in the XF96 cell culture plate in $200 \mu \mathrm{L}$ growth medium per well and cultured in normal growth conditions overnight. Furthermore, sensor cartridges were hydrated by adding $200 \mu \mathrm{L}$ of XF calibrant to the $\mathrm{XF}^{\mathrm{e}} 96$ sensor cartridge plate and incubated in a non- $\mathrm{CO}_{2}$ incubator at $37^{\circ} \mathrm{C}$ overnight. On the day of the assay, XF medium was warmed, the $\mathrm{pH}$ was set to 7.4 and supplemented with $25 \mathrm{mM}$ glucose. The previously cultured cells in the XF96 cell culture plate were washed with warm PBS and the medium was replaced with $180 \mu \mathrm{L}$ of warm XF medium and incubated at $37^{\circ} \mathrm{C}$ for 1 hour without $\mathrm{CO}_{2}$. The following compounds were prepared in warm XF medium and loaded in the indicated ports in the sensor cartridge using the templates provided: $10 \mu \mathrm{M}$ Oligomycin $(20 \mu \mathrm{L}$ per well loaded in Port A), $20 \mu \mathrm{M}$ FCCP $(22 \mu \mathrm{L}$ per well loaded in Port B) and $10 \mu \mathrm{M}$ each of Rotenone and Antimycin (25 $\mu \mathrm{L}$ per well loaded in Port C). The sensor cartridge plate was loaded and calibrated in the XF96 Extracellular Flux Analyzer, which was always switched on at least 5 hours prior to assay. Following calibration, the cell culture plate was loaded into the XF96 Extracellular Flux Analyzer and a MitoStress Test was run using the $\mathrm{XF}^{\mathrm{e}}$ analyzer software. Data were exported as a Microsoft Excel file for further analyses. 
Protein amount was measured following assay completion using the Pierce BCA microassay as described (see 2.2.6.3) for normalization purposes.

\subsubsection{Confocal microscopy and image analyses}

Images of frozen brain sections were acquired using the LSM 800 with Airyscan setup (Zeiss). The same background and gain settings were maintained for all compared groups. Images were acquired with the 20X Plan Apochromat objective (Zeiss). All settings were saved and applied to subsequent imaging sessions for comparable groups. Post-acquisition image analyses were carried out with ZEN software (Zeiss) and Image J.

\subsubsection{Bioinformatics}

With the advent of large-scale multi-omics data, bioinformatics provides tools to accurately analyze biological data and thus make reliable predictions of the structure, networks, and function of macromolecules. These tools were extensively relevant in realizing the goals of this study. The next few sections describe some of these tools and how useful they were in this study.

\subsubsection{Microarray dataset mining and analyses}

In order to identify transcriptional signatures mediating organelle crosstalk in lysosomal malfunction, microarray data involving lysosomal malfunction were mined from transcriptome dataset repository, Gene Expression Omnibus (http://www.ncbi.nlm.nih.gov/geo). Criteria for dataset selection included datasets from several tissues with multiple replicates. The selected dataset was GSE39621, which included samples from brain, liver, and spleen of NPC1/- mice and their wild-type littermates before and after 6 weeks of age, a relevant time point for the onset of disease manifestation (Alam et al., 2012). Splenic datasets were excluded from this study given the heterogeneous population of cells (e.g. immune cells) that invade the spleen in this disease (Patterson et al., 2012). It was, therefore, reasoned that wild-type and NPC1\% splenic datasets were not directly comparable. As a result, brain and liver datasets were loaded into the microarray data analysis software GeneSpring 13.0 (Agilent Technologies) and datasets were normalized by robust multi-array averaging (Irizarry et al., 2003). Following normalization, differentially expressed genes (DEG) were determined as statistically significant 
genes between conditions at a cut-off threshold of $p$-value $<0.05$ using ANOVA with a Bonferroni post hoc correction.

\subsubsection{Pathway analyses and identification of transcriptional regulators}

The DEGs were imported into the comprehensive pathway analyses software Ingenuity Pathway Analysis (IPA, Qiagen) in order to identify signaling pathways responding to lysosomal malfunction. IPA determines statistically enriched transcriptional regulators and signaling pathways using the Fisher's exact test. The statistical cut-off was set at $p<0.01$.

\subsubsection{Organelle-specific gene expression analyses}

In order to determine the average expression of organelle-specific genes, which we showed previously to be a good indicator of the biogenesis of that organelle (Fernández-Mosquera et al., 2017), we obtained organelle proteomes from comprehensive databases for lysosomal, mitochondrial (and respiratory chain subunits), peroxisomal, endoplasmic reticulum and Golgi proteomes (see Table 14). The protein IDs were converted to NCBI gene symbols. These genes were translated to their corresponding probeset IDs in GeneSpring 13.0 and their expression levels in the GSE39621 datasets were obtained and exported as Microsoft Excel files. The average expression of organelle gene list was calculated as the average cumulative fold change of all genes that make up each organelle. Differences between wild-type and NPC1 ${ }^{-1}$ organelle expression were considered significant if they passed a statistical threshold of $p$-value $<0.05$ determined by Student's $t$-test with Bonferroni post hoc correction.

Table 14: Source of organelle-specific proteome database

\begin{tabular}{lcl}
\hline \hline Dataset & Number of genes & Source \\
\hline Mitochondria & 1049 & (Calvo et al., 2016; Pagliarini et al., 2008) \\
Respiratory chain subunits & 108 & (Calvo et al., 2016; Pagliarini et al., 2008) \\
Lysosomes & 435 & (Brozzi et al., 2013) \\
Peroxisomes & 254 & (Wiese et al., 2007) \\
Endoplasmic reticulum & 297 & (Gilchrist et al., 2006) \\
Golgi (COP I) vesicles & 86 & (Gilchrist et al., 2006) \\
\hline \hline
\end{tabular}




\subsubsection{Promoter analyses of Respiratory chain genes}

Promoter analyses were carried out to identify which transcription factors (TFs) mediated the expression of respiratory chain genes. This was performed using the software Genomatix Suite (http://www.genomatix.de), which provides a robust tool for predicting TFs that drive gene expression. Promoter regions of respiratory chain genes were first defined from 500 base pairs upstream $(-500)$ until 100 base pairs downstream $(+100)$ of the transcription start site (TSS). To locate these promoters, we used the Genomatix tool Gene2Promoter and subsequently identified the TFs which had binding sites on them. Only promoters that drive the expression of mRNAs which lead to fully functional respiratory chain proteins were selected for each gene. The promoter sequences were used to determine cis-elements and identify the corresponding TFs. TFs with binding sites in less than $85 \%$ of the promoters were excluded. In addition, a statistical threshold of $p$-value $<0.05$ was set for significantly enriched TF families determined by the Fisher's exact test within the software suite. Within the enriched TF families, only individual TFs with binding sites in at least $50 \%$ of promoters under analyses were selected as relevant.

\subsubsection{ChIP-Seq dataset mining and analyses}

In order to validate the role of ETV1 as a regulator of mitochondrial biogenesis, we obtained published ETV1 ChIP-Seq data (Baena et al., 2013). Genes with promoter regions bound exclusively by ETV1 and not its related transcription factor family member ERG were selected with a MAT score threshold of $p<0.0001$. This gene list was crossed with our mitochondrial gene list (see section 2.2.9.3) to obtain mitochondrial genes under transcriptional regulation of ETV1.

\subsubsection{Metabolomics}

Lysosomes and mitochondria serve as distinct hubs for several metabolic processes in the cell and metabolite profiling provides unique fingerprints of such processes. To obtain such features, stable GAA knockdown cells and their scrambled controls were cultured under normal growth conditions until $100 \%$ confluence. The cells were washed with ice-cold PBS, and all metabolic process were quenched by harvesting cells in $500 \mu \mathrm{L}$ cold $50 \%$ MS-grade methanol with a scraper. The collected cells were transferred into an Eppendorf tube containing $500 \mu \mathrm{L}$ MS-grade chloroform on ice. The samples were snap-frozen in liquid nitrogen and sent to a 
metabolomics facility at the Department of Plant Biochemistry, Goettingen Center for Molecular Biosciences. At the facility, briefly, the samples were dried under a nitrogen gas stream and the metabolites were extracted in extraction solvent (a mixture of methanol, chloroform, and water, 129:50:25 [v/v/v]). Following addition of internal standards, metabolites were derivatized by methoxyamination and trimethylsilation. Derivatized metabolites were subjected to gas chromatography coupled to a quadrupole mass spectrum analyzer. The identities of metabolites were determined by comparing spectra to those of a comprehensive metabolite library. The abundance of each identified metabolite in each sample was determined as its concentration. The results were exported as Microsoft Excel files along with the weight of each sample for normalization purposes. The data were loaded into MetaboAnalyst 3.0 (www.metaboanalyst.ca) where features whose concentrations were significantly altered $(p<$ $0.05)$ between scrambled and GAA knockdown cells were determined. The altered metabolites were subsequently used for metabolic pathway and metabolite set enrichment analyses.

\subsubsection{Magnetic resonance spectroscopy and imaging}

$\mathrm{GAA}^{-/}$and control mice underwent magnetic resonance spectroscopy, $\mathrm{T}_{1}$ and $\mathrm{T}_{2}$ relaxation times of water protons measurements and magnetization transfer ratio determinations of the cortex, thalamus, and striatum at $37^{\circ} \mathrm{C}$. Magnetic resonance images of the whole brain of mice were also obtained. All nuclear magnetic resonance (NMR) experiments were carried out with the support of Takashi Watanabe at the Biomedical NMR Department of the Max-Planck Institute for Biophysical Chemistry following the protocol described in Watanabe et al. (2016).

\subsubsection{Statistical analyses}

Unless otherwise indicated, results were analyzed using Microsoft Excel 2013 and GraphPad Prism versions 6 and 7. Graphs were presented as mean values normalized to experimental controls and their corresponding standard errors of the mean for at least three biological replicates and replicate experiments. Differences of means were considered statistically significant if they passed thresholds calculated as Student's $t$-test for two-parameter comparisons, or as ANOVA for multi-parametric comparisons. These were indicated as $p<$ $\left.0.05\left(^{*}\right), \mathrm{p}<0.01^{(* *}\right)$ or $\left.\mathrm{p}<0.001^{(* *}\right)$. 


\section{Results}

\subsection{Impaired sphingosine-1-phosphate signaling in Niemann-Pick disease represses mitochondrial biogenesis and function via KLF2 and ETV1}

\subsubsection{Lysosomal dysfunction in Niemann-Pick patient fibroblasts}

Although mitochondrial defects have previously been reported in the different types of Niemann-Pick diseases (Castillo et al., 2017; Woś et al., 2016), the mechanisms linking dysfunctional lysosomes to defective mitochondria remained nevertheless elusive. To address this in the context of a lysosomal-mitochondrial crosstalk, Niemann-Pick Type A/B (hereafter NP) and age-matched control fibroblasts were obtained from the UMG. The NP fibroblasts retained about $15 \%$ of acid sphingomyelinase activity and impaired lysosomal function was validated by assessing autophagy and lysosomal proteolytic capacity, which were found to be compromised (Figure 12).
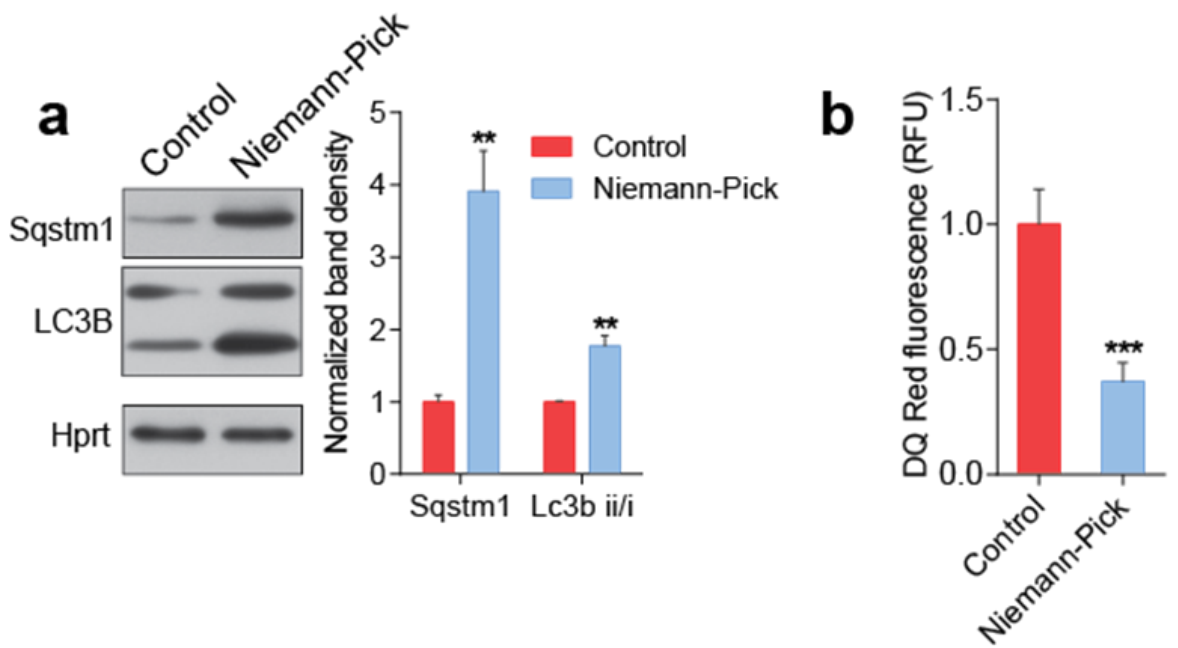

Figure 12| Lysosomal function is impaired in NP fibroblasts - A NP cells present stalled autophagy with accumulation of autophagosomal marker LC3B and autophagic substrate SQSTM1. B Lysosomal proteolytic capacity, measured by the Red DQ-BSA assay, is reduced in NP fibroblasts. Graphs represent mean \pm SD of at least biological triplicates. Differences between groups were considered statistically significant based on Student's t-test at $\mathrm{p}<0.01\left(^{* *}\right)$ or $\mathrm{p}<$ $\left.0.001{ }^{* * *}\right)$, SD: standard deviation 


\subsubsection{Mitochondrial function is compromised in NP fibroblasts}

Having confirmed lysosomal impairment in NP fibroblasts, mitochondrial function was then assessed. To this end, mitochondrial oxygen consumption rate (OCR) was measured under basal conditions and upon inhibition or uncoupling of oxidative phosphorylation (maximal OCR). The NP fibroblasts presented reduced basal and maximal OCR (Figure $13 \mathrm{~A}$ and B). In addition, about a two-fold increase in mitochondrial superoxide steady-state levels was observed in the NP fibroblast as determined by MitoSox fluorescence (Figure 13C).
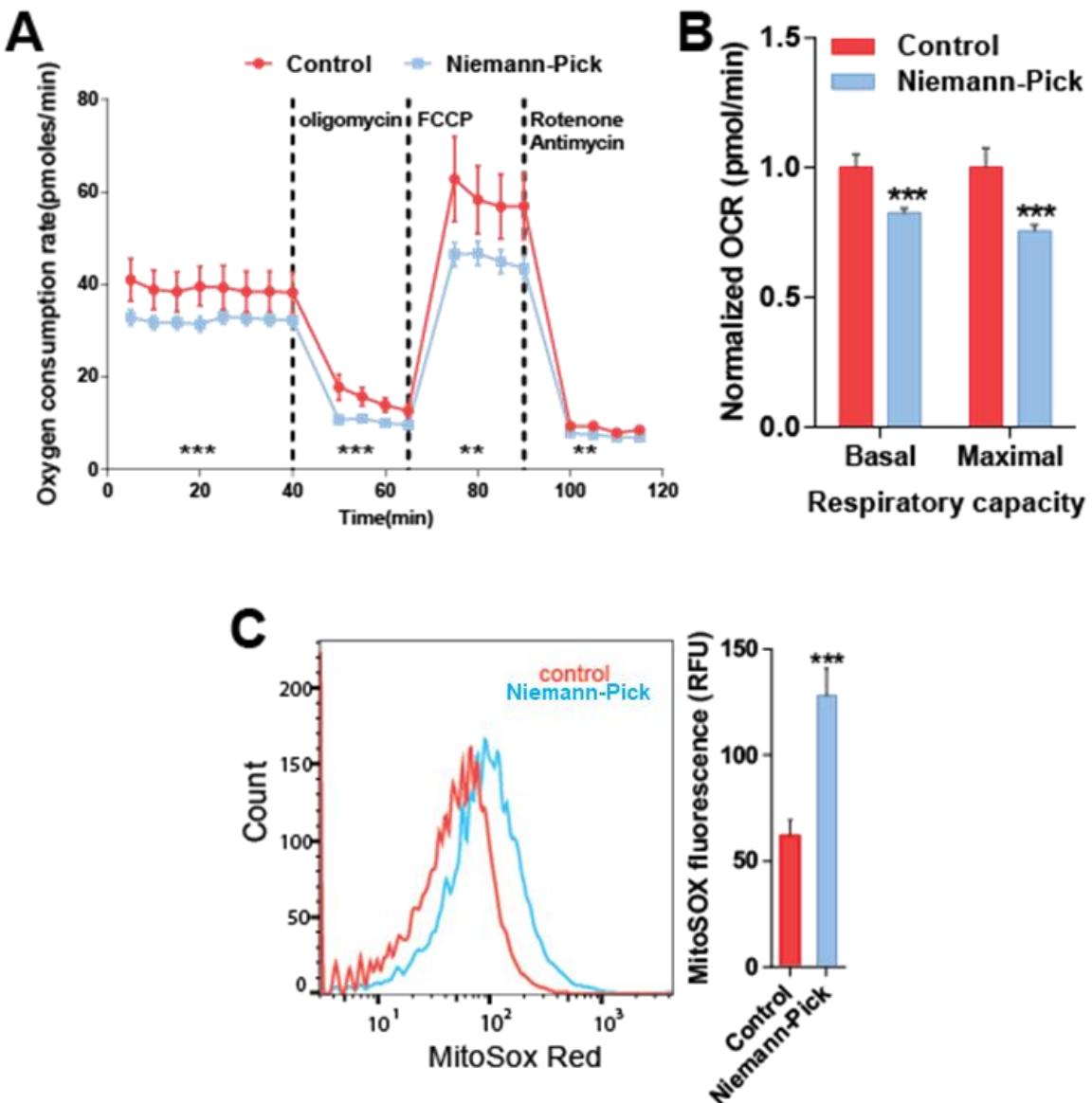

Figure 13| Mitochondrial malfunction in NP fibroblasts - $\mathbf{A}$ and $\mathbf{B}$ Reduced basal and maximal mitochondrial OCR in NP fibroblasts as assessed by Real Time respirometry $(\mathrm{N}=8)$. C Increased MitoSox fluorescence in NP fibroblasts, which reflects mitochondrial superoxide levels $(\mathrm{N}=5)$. Graphs are mean \pm SEM for the indicated number of replicate samples, $\mathrm{p}<0.001$ $\left.{ }^{* * *}\right)$; Student's t-test; SEM: standard error of the mean 

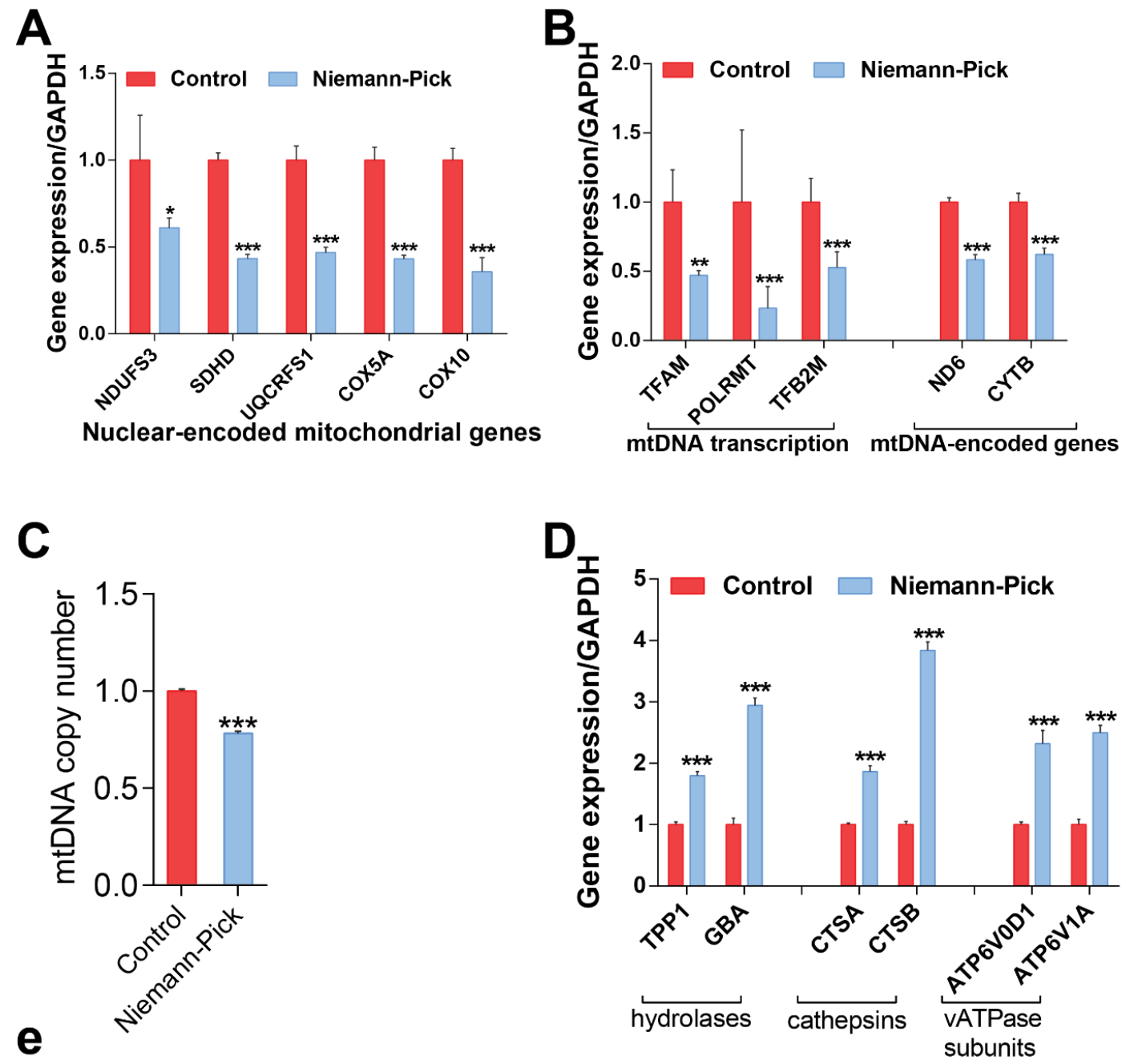

Figure 14| Mitochondrial biogenesis is repressed in NP fibroblasts - A Reduced expression of nuclear-encoded mitochondrial genes in NP. B Transcript levels of regulators of mtDNA transcription and mtDNA-encoded respiratory chain genes are reduced in NP. C Reduced mtDNA copy number in NP fibroblasts. D Unlike mitochondrial biogenesis, lysosomal biogenesis is up-regulated in NP. Gene expression is depicted as mean $\pm \mathrm{SEM}, \mathrm{N}=8$. Based on ttest, differences were significant at $\mathrm{p}<0.05\left({ }^{*}\right)$ and $\mathrm{p}<0.001\left({ }^{* * *}\right)$, SEM: standard error of the mean

\subsubsection{Repression of mitochondrial biogenesis in NP fibroblasts}

Mitochondrial biogenesis is known to be coupled to oxygen consumption and the production of ATP (LeBleu et al., 2014). Given the observed reduced OCR in NP fibroblasts, it was reasoned that mitochondrial biogenesis may be repressed. As a result, the expression of several 
mitochondrial genes and regulators of transcriptional programs of mitochondrial biogenesis was measured. Importantly, the expression of several nuclear-encoded respiratory chain subunits including NDUFS3, SDHD, UQCRFS1, COX5A, and COX10 were decreased (Figure 14A). Furthermore, the transcript levels of essential regulators of mtDNA maintenance and transcription (TFAM, POLRMT, and TFB2M) and mtDNA-encoded respiratory chain genes (ND6, CYTB) were also downregulated in NP fibroblasts (Figure 14B). Not surprisingly, this transcriptional response culminated in a loss of mtDNA copy number in NP (Figure 14C). The evidence of repression of mitochondrial biogenesis was further supported by reduced protein levels of NRF1, a major transcriptional regulator of mitochondrial biogenesis (Kelly and Scarpulla, 2004; Scarpulla, 2008) and its target TFAM, a key regulator of mtDNA maintenance and gene expression (Gustafsson et al., 2016), as shown in Figure 15. To rule out bias in the measurement of mitochondrial transcript levels that were all decreased in NP, the expression of lysosomal genes which are known to be up-regulated in LSDs (Karageorgos et al., 1997) was measured.

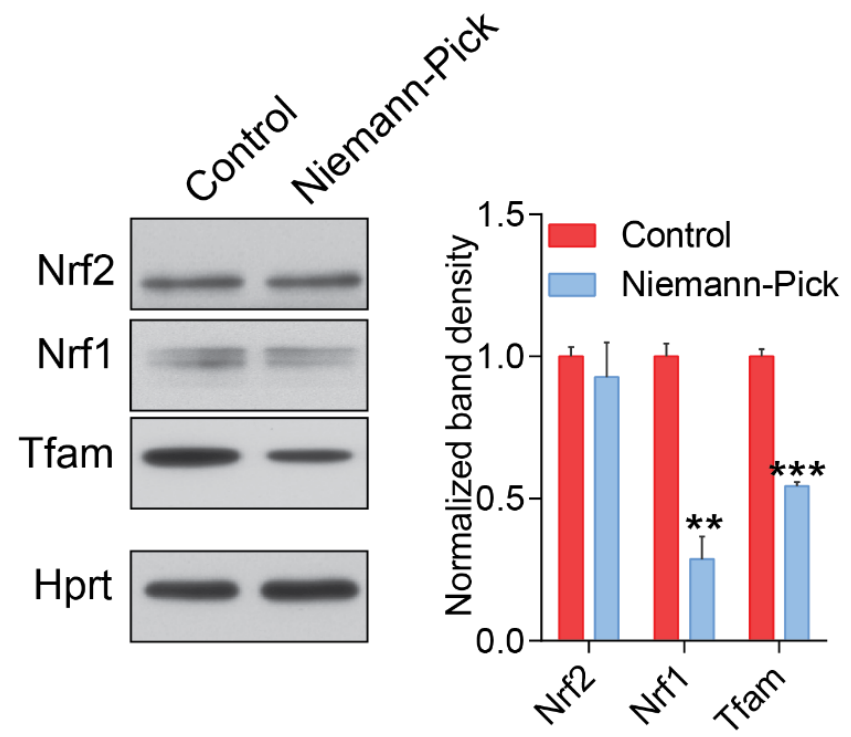

Figure 15| Levels of regulators of mitochondrial biogenesis - Protein amounts of NRF1, NRF2, and TFAM, which are key regulators in coordinating mitochondrial biogenesis were measured by WB. Quantifications of bands are depicted as mean $\pm \mathrm{SD}, \mathrm{N}=3 . \mathrm{p}<0.01$ ( $^{* *}$ ) and $\left.\mathrm{p}<0.001{ }^{* * *}\right)$; Student's t-test; SD: standard deviation 


\subsubsection{Desipramine treatment impairs lysosomal function}

In order to validate these results and to rule out off-target effects of SMPD1 mutations in patients, control fibroblasts were treated with desipramine, an inhibitor of acid sphingomyelinase (ASM), the affected enzyme in NP. Desipramine treatment has been shown to result in a time-dependent loss of ASM activity by targeting the enzyme for proteolysis (Jenkins et al., 2011). Following 72 hours of treatment of control fibroblasts with $40 \mu \mathrm{M}$ desipramine, impaired lysosomal function, similar to that in NP fibroblasts, was found as measured by increased autophagosomal mass and stalled autophagy (Figure 16A) and reduced lysosomal proteolytic capacity (Figure 16B).
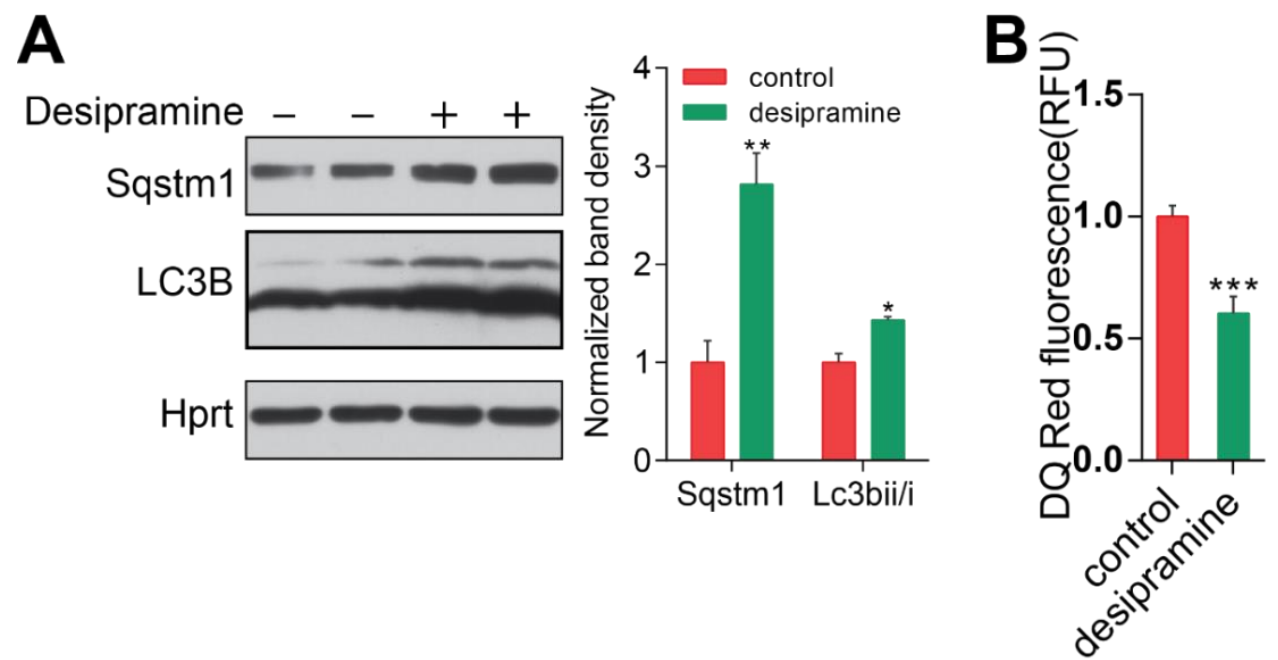

Figure 16| Lysosomal function is impaired in desipramine-treated cells - A Increased levels of LC3B and SQSTM1 reflect stalled autophagy in desipramine-treated fibroblasts. B Desipramine treatment impairs lysosomal proteolysis. Graphs are mean \pm SEM for at least triplicate samples and differences were significant based on the t-test, $\mathrm{p}<0.05\left(^{*}\right), \mathrm{p}<$ $0.01\left(^{* *}\right), \mathrm{p}<0.00\left(^{* * *}\right)$, SEM: standard error of the mean

\subsubsection{Desipramine treatment represses mitochondrial biogenesis and function}

Following confirmation that desipramine treatment in cells affected lysosomes similarly to NP, mitochondrial function was assessed. Marked reductions in basal and maximal mitochondrial OCR and increased mitochondrial superoxide levels were found (Figure $17 \mathrm{~A}, \mathrm{~B}$ and $\mathrm{C}$ ). Furthermore, like in NP fibroblasts, desipramine treatment resulted in decreased expression of several nuclear- and mtDNA-encoded mitochondrial genes (Figure 17D) and increased 
lysosomal biogenesis (Figure 17E). Altogether, pharmacological ASM inhibition corroborates the results obtained in NP fibroblasts, and also rules out SMPD1 mutation-specific effects.
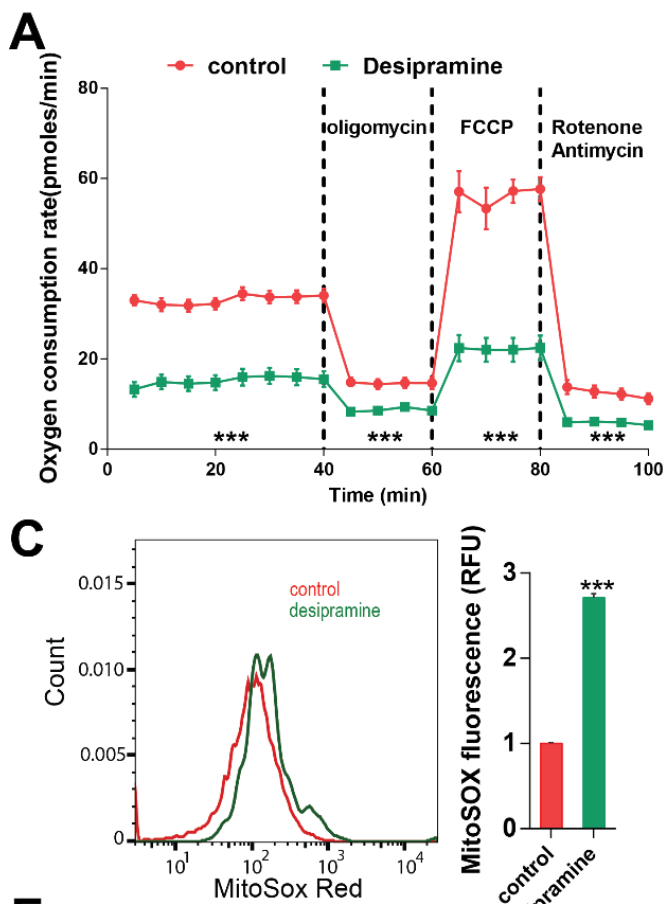

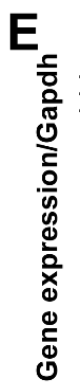
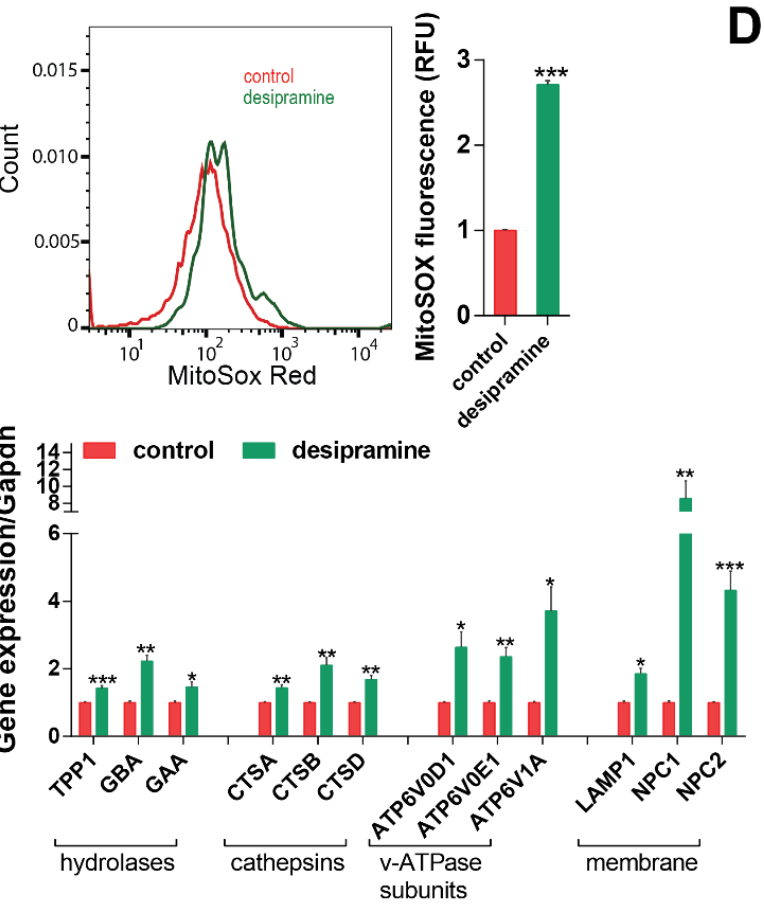

Lysosomal genes
B

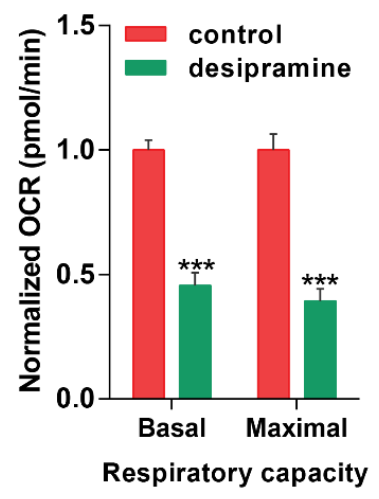

D

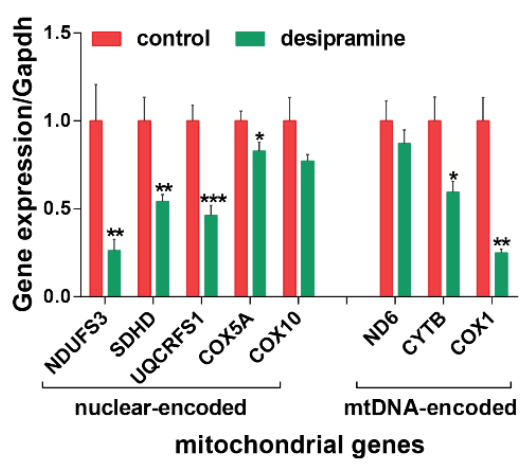

Figure 17| Desipramine treatment represses mitochondrial biogenesis and

function - A and B Cells treated with desipramine show decreased mitochondrial respiration.

C Increased mitochondrial superoxide levels in desipramine treated cells. D Treatment with desipramine reduces the expression of mitochondrial genes. $\mathbf{E}$ Lysosomal biogenesis is increased in desipramine-treated cells. Graphs are shown as mean \pm SEM, N $=8 ; p<0.05\left(^{*}\right), p<$ $\left.0.01{ }^{* *}\right)$, and $\mathrm{p}<0.001\left(^{* * *}\right) ;$ Student's t-test; SEM: standard error of the mean 


\subsubsection{Transcriptomics reveal global downregulation of mitochondria-related} genes in NPC1/- mouse tissues

Although our results consistently showed that mitochondrial biogenesis was repressed in NP patient fibroblasts as well as in desipramine-treated cells, it is noteworthy that only a small fraction of mitochondrial genes was studied. We, therefore, set out to corroborate these data using groups of mitochondrial or lysosomal genes as comprehensively as possible. To this end, bioinformatics approaches developed in the lab were used to analyze publicly available transcriptome datasets of $\mathrm{NPC}^{-1}$ mice, a model of Niemann-Pick type $\mathrm{C} 1$ disease. This approach is illustrated in Figure 18. A detailed description of the approach and microarray dataset can be found in section 2.2.9.

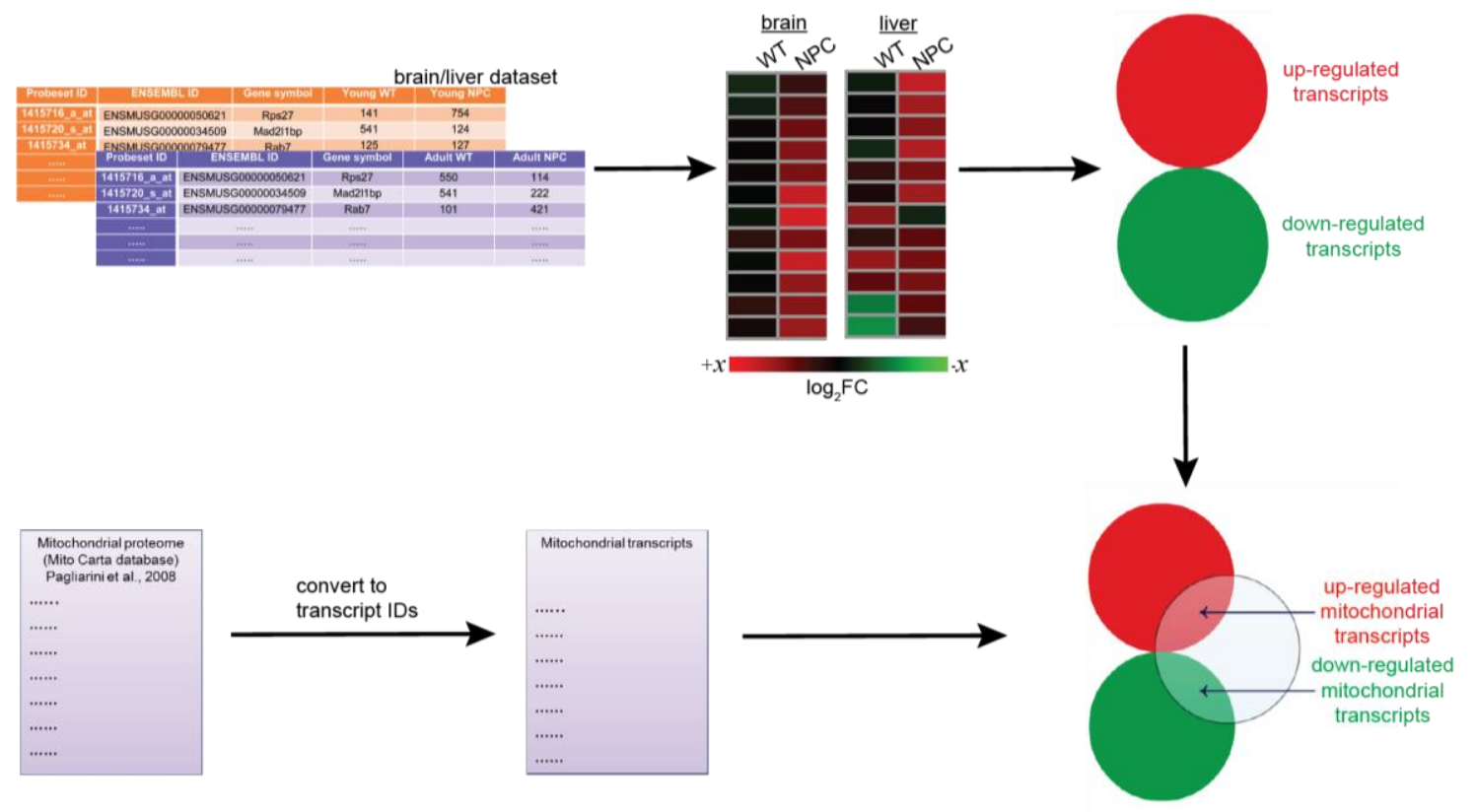

Figure 18| Schematic representation of genomics approach for in silico experiments - Differentially-expressed genes (DEGs) were filtered with organelle-specific gene lists to obtain organelle-related DEGs

Lysosomal biogenesis was assessed by measuring the average expression of lysosomal genes in $\mathrm{NPC}^{-1}$ mice relative to their control littermates, which was found to be increased in all tissues and ages tested (Figure 19A). This corroborated our results in NP fibroblasts, and have validated our transcriptome data analyses approach. 

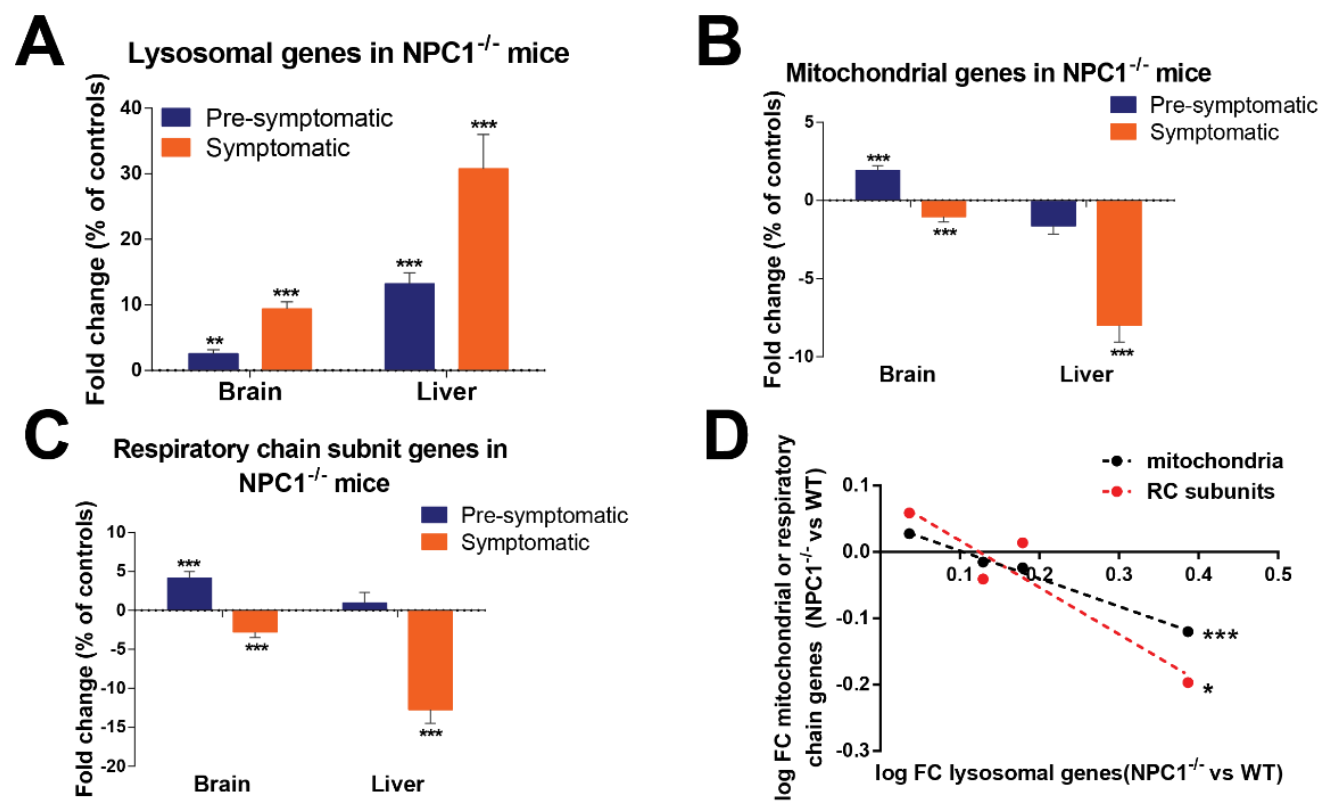

Figure 19| Differentially expressed organelle genes in NPC1\%-mice - A Lysosomal gene expression is increased in NPC1\% mice. $\mathbf{B}$ and $\mathbf{C}$ Mitochondrial and respiratory chain genes are decreased with the onset of disease in NPC $\%$ mice. D Illustration of the correlation of gene expression between lysosomes and mitochondria and respiratory chain subunits. Differences in organelle expression between wildtype and NPC1\%.mice passed statistical thresholds determined by the Students t-test with Bonferroni post-hoc correction at $\mathrm{p}<0.05\left(^{*}\right), \mathrm{p}<0.01$ $\left({ }^{* *}\right)$, and $\mathrm{p}<0.001\left(^{* * *}\right)$. Graphs represent normalized mean \pm SEM, $\mathrm{N}=6$; SEM: standard error

Similarly, the average expression of mitochondrial-related genes in the brain and in the liver of $\mathrm{NPC}^{-1}$ mice was measured. While pre-symptomatic mice showed an increase in the expression of mitochondrial genes in the brain and no significant change in the liver, symptomatic mice, on the other hand, showed a robust downregulation in the average expression of mitochondrial genes (Figure 19B). The pattern of expression of OXPHOS-related genes in the tissues was similar to the expression of mitochondrial genes albeit with a rather larger magnitude (Figure 19C). To exclude the possibility that a few dysregulated genes might be driving the pattern of expression of mitochondrial and OXPHOS related genes that were observed in the symptomatic mouse tissues, the proportion of organelle-specific DEGs before and after the onset of symptoms was assessed. Indeed, the proportion of mitochondrial DEGs was increased in the brain and in the liver after disease onset (Figure 20). 
Having observed increased expression of lysosomal genes and reduced expression of mitochondrial and OXPHOS related genes after disease onset, we sought to establish a tissueindependent general pattern of gene expression between lysosomes and mitochondria. As a result, the level of significance of the negative correlation of gene expression between these organelles was tested and found to be significant for both mitochondria and OXPHOS (Figure 19D). Taken together, these results confirm our findings that lysosomal malfunction progressively represses mitochondrial biogenesis.

A

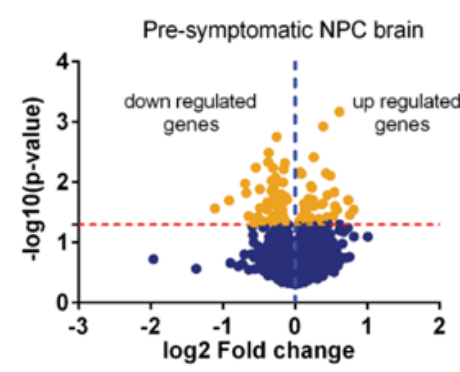

D

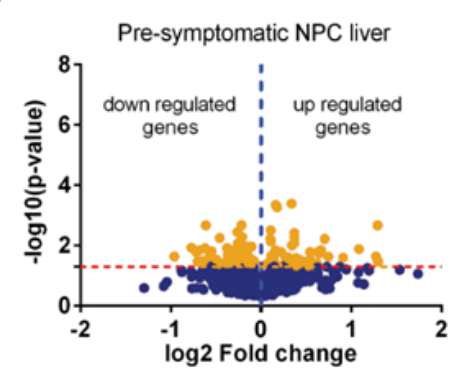

B

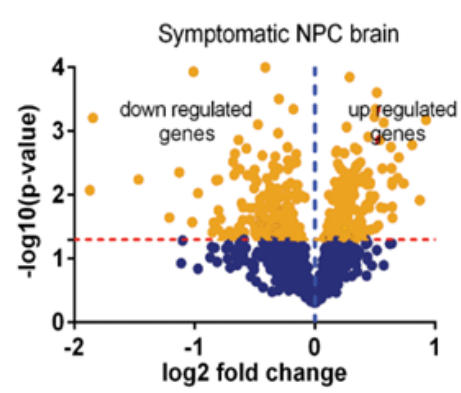

E

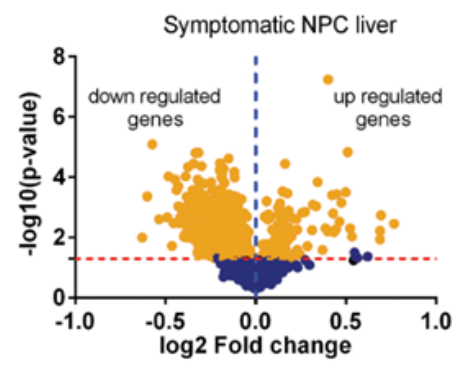

C

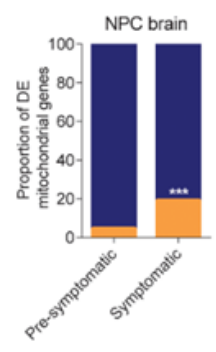

$\mathbf{F}$

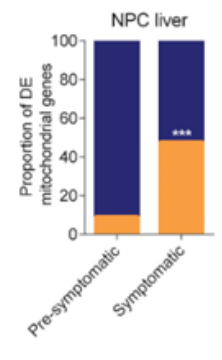

Figure 20| Disease progression changes in mitochondrial gene expression A - C Disease progression-dependent increase in mitochondrial DEGs in NPC1\% mouse brain. D - F NPC1 $\%$ mouse liver shows a five-fold increase in the proportion of mitochondrial DEGs with disease progression. Tests for differences in proportions were statistically significant at $\mathrm{p}$ $<0.001\left(^{* * *}\right)$

To verify whether the transcriptional response to lysosomal malfunction was specific to mitochondria, the average expression of genes related to peroxisomes, endoplasmic reticulum (ER) and Golgi were measured. Irrespective of disease onset, it was found that the average expression of peroxisomal-, ER-, and Golgi-related genes was not changed in the brain of $\mathrm{NPC1}^{-/-}$mice (Figure 21). 


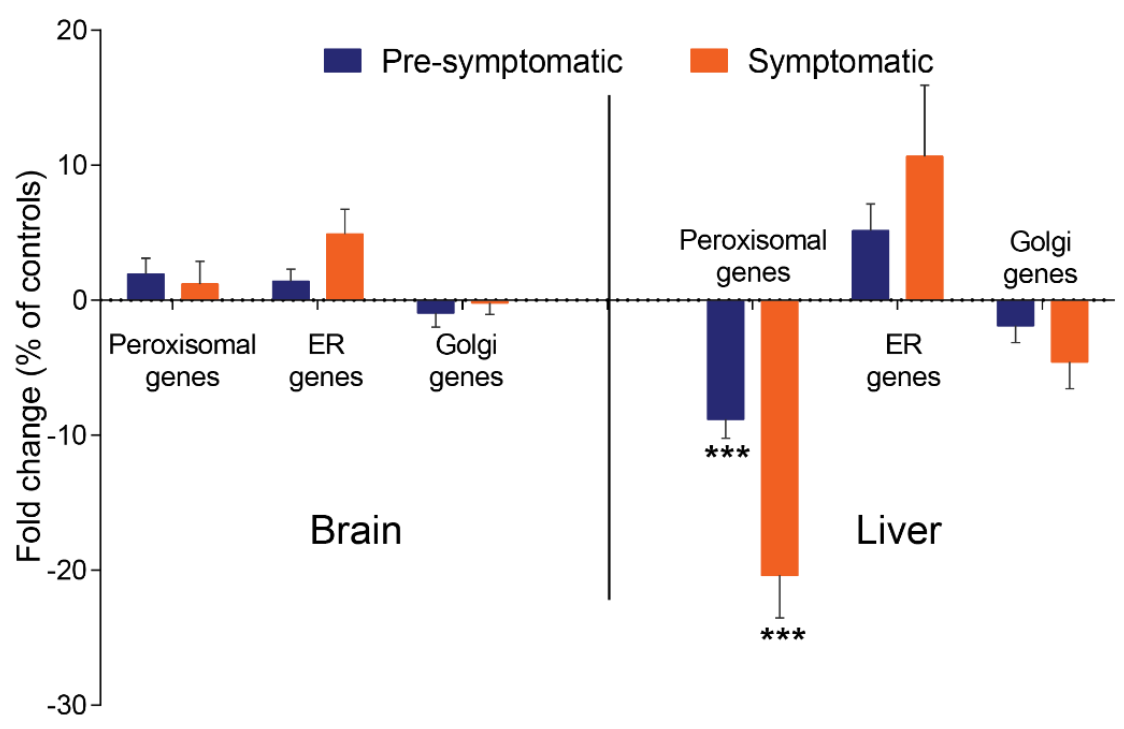

Figure 21| Organelle-specific gene expression changes in NPC1\%- mice - NPC1\% mice show no changes in the expression of ER and Golgi genes. However, peroxisomal genes are robustly downregulated in the liver and not altered in the brain. Differences in organelle expression between wildtype and NPC1\%.mice passed statistical thresholds determined by the Students t-test with Bonferroni post-hoc correction at $\mathrm{p}<0.001\left(^{* * *}\right)$. Graphs represent normalized mean \pm SEM, N =6, SEM: standard error

While a similar pattern of expression was observed for ER- and Golgi-related genes in the liver, peroxisomal related genes were significantly downregulated in the liver (Figure 21). These results underscore a specific mitochondrial response to lysosomal malfunction in $\mathrm{NPC}^{-/-}$mice.

\subsubsection{Reduced mitochondrial respiration in NPC1//- MEFs}

Having demonstrated that mitochondrial biogenesis is repressed in NPC1/- mouse tissues, especially after the onset of disease, we postulated that these deficits will compromise mitochondrial respiration, which is tightly linked to mitochondrial biogenesis (LeBleu et al., 2014). Mitochondrial OCR was therefore assessed in MEFs obtained from NPC1 ${ }^{-/}$mice under basal conditions and upon inhibition or uncoupling of OXPHOS. Not surprisingly, decreased basal and maximal mitochondrial respiratory capacities were observed in the NPC1/- MEFs when compared to their wild-type controls (Figure $22 \mathrm{~A}$ and $\mathrm{B}$ ). These findings altogether show that lysosomal malfunction in Niemann-Pick disease, irrespective of the type, elicits a 
transcriptional program of repressed mitochondrial biogenesis in vitro and in vivo, which ultimately impairs mitochondrial function.
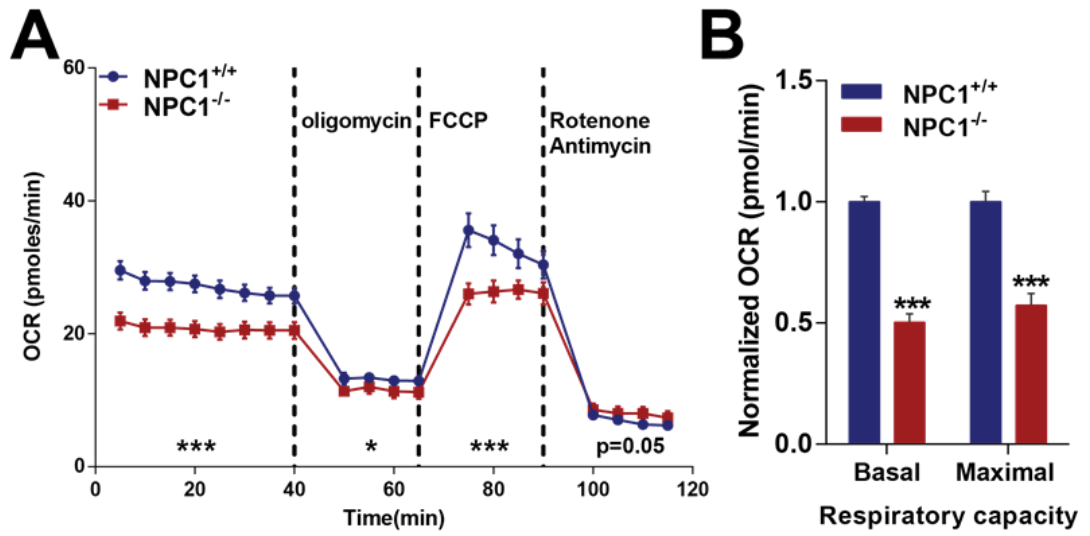

Figure 22| Mitochondrial respiration is reduced in NPC1\%- MEFs - A Mitochondrial respiration profile shows reduced OCR in NPC1\% MEFs. B Basal and maximal mitochondrial respiratory capacity in NPC1\% cells are decreased to about $50 \%$ of wild-type cell levels. Graphs represent mean \pm SEM for $\mathrm{N}=8$; $\mathrm{p}<0.001\left(^{* * *}\right)$; Student's t-test; SEM: standard error of the mean

\subsubsection{KLF2 and ETV1 mediate repression of mitochondrial biogenesis in}

\section{Niemann-Pick disease}

In addition to showing that lysosomal malfunction in Niemann-Pick disease specifically represses mitochondrial biogenesis, we were further interested in elucidating the mechanism(s) which mediate such a response. Additionally, the global decrease in

mitochondrial gene expression in all conditions tested suggested the involvement of a coordinated transcriptional program. To address this, promoter analyses of the respiratory chain genes was carried out using the MatInspector software tool (Genomatix). The process is described in detail in section 2.2.9.4. Table 15 shows transcription factor families with significantly enriched binding sites in the promoters of respiratory chain genes. SP1 and E2F transcription factor families were eliminated from further analysis since they were predicted to have statistically enriched binding sites in the promoters of lysosomal genes. Given the significant negative correlation of gene expression between lysosomes and mitochondria/ respiratory chain in Niemann-Pick disease, it was reasoned that it is unlikely that SP1 and E2F transcription factor families mediated antagonistic processes. Hence, only the RC gene- 
specific KLF and ETS transcription factor families were subsequently studied in the NiemannPick disease models.

Table 15: Transcription factors with enriched cis-elements in RC gene promoters

\begin{tabular}{lll}
\hline \hline Transcription factor family & p-value (Fisher's exact test) & Transcription factors \\
\hline SP1 & $1.52 \times 10^{-9}$ & SP1, SP4 \\
E2F & $2.79 \times 10^{-8}$ & E2F1, E2F2, E2F3, E2F4 \\
KLF & 0.000265 & KLF2, KLF6, KLF7, KLF15 \\
ETS & 0.000796 & ELK1, SPI1, ETV1 \\
\hline \hline
\end{tabular}

Since it is unlikely that all transcription factors in these families would be active or repressed at the same time to mediate one response in Niemann-Pick disease, the number of transcription factors in each family were trimmed using the transcriptome dataset of $\mathrm{NPC}^{-/}$mice. Ingenuity pathway analyses of the DEGs in the microarray data (described previously see section 2.2.9) scored active/repressed TFs in the NPC1/- mouse tissues. The active/repressed TF dataset was crossed with the ones regulating respiratory chain genes, and only KLF2 and SPI1 were found to pass the set criteria (Figure 23A).
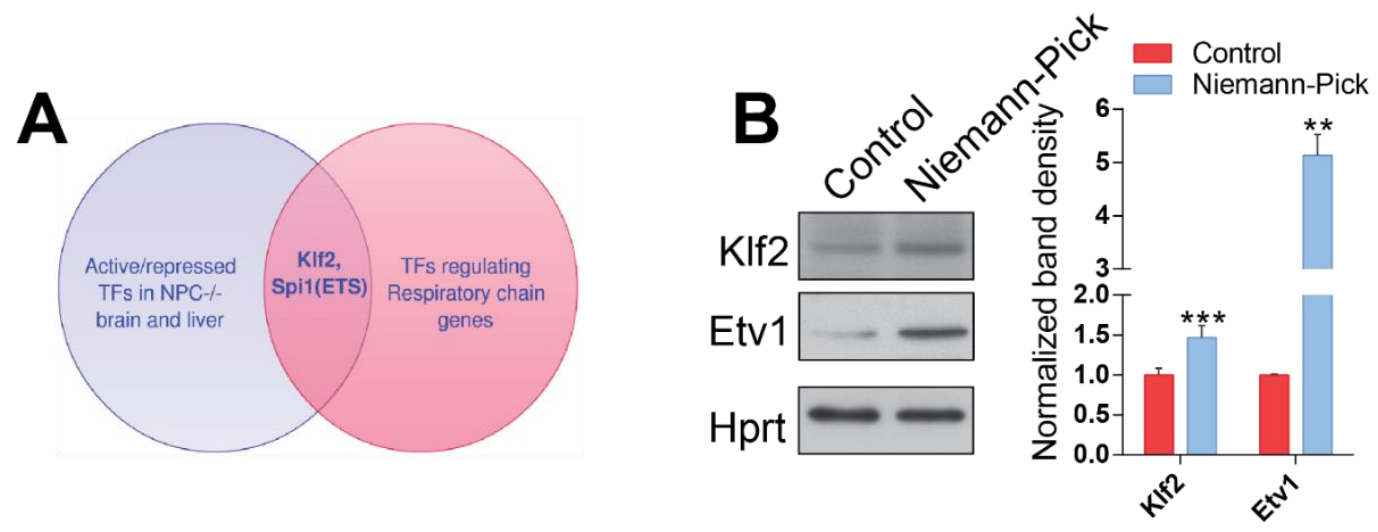

Figure 23| Transcriptional regulation of Respiratory chain genes - A Schematic illustration of the TF filtering for those relevant for RC gene expression. B KLF2 and ETV1 are significantly induced in NP fibroblasts .Band quantifications are depicted as mean $\pm S E M, N=5$. Differences between means, which passed the statistical threshold of $\mathrm{p}<0.01\left(^{* *}\right)$ and $\mathrm{p}<0.001$ $\left({ }^{* *}\right)$ based on the Student's t-test, were considered significant; SEM: standard error of the mean 
Furthermore, about $50 \%$ increase in the protein amount of KLF2 was found in NP fibroblast (Figure 23B). SPI1, either at transcript or at protein levels, could not be detected in NP fibroblasts. This is corroborated by reports that SPI1 is expressed in macrophages and not in fibroblasts (Feng et al., 2008). The ETS family of TFs have been shown to mostly have redundant functions (Maroulakou and Bowe, 2000). To this end, two other commonly expressed members of this family were tested. While a two-fold increase in the transcript level of ETV1 was observed in NP fibroblasts, ELK1 expression was unaltered (see Appendix Figure 52). Accordingly, a five-fold increase in ETV1 protein in NP fibroblasts was found (Figure 23B). To further validate the results of our promoter analyses, publicly-available ETV1 ChIP-Seq data (Baena et al., 2013) were crossed with our mitochondrial gene list, and ETV1 was demonstrated to regulate the transcription of several mitochondrial genes (Figure 24). In addition, KLF2 and ETV1 were strongly induced in fibroblasts treated with desipramine (see Appendix Figure 53).

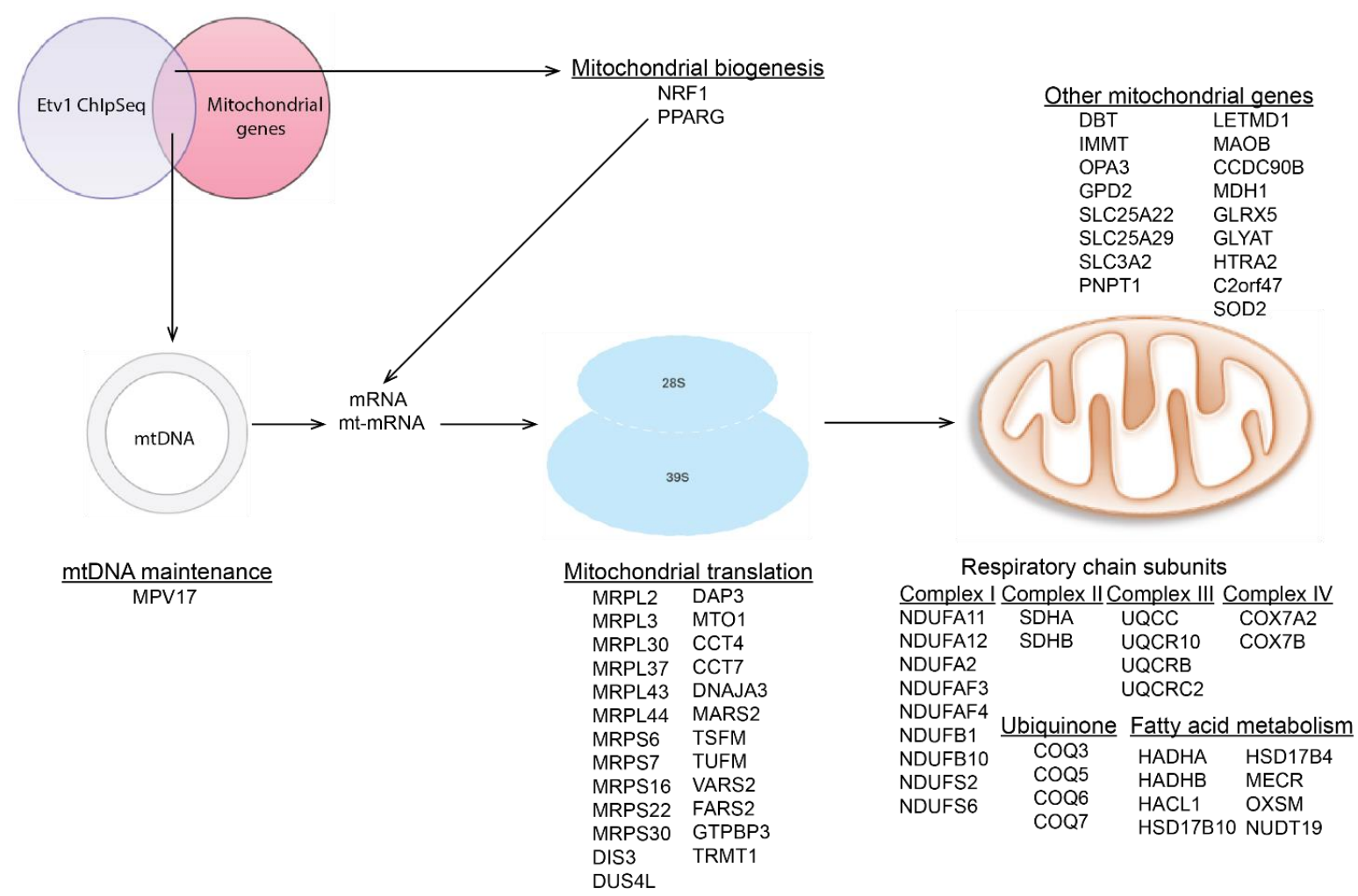

Figure 24| ETV1 targets mitochondrial genes - Schematic representation of ETV1 ChIPSeq data analyses reveals several mitochondrial genes as targets of ETV1 
We reasoned therefore that KLF2 and ETV1 might be mediating the repression of mitochondrial biogenesis, since they were both induced in NP fibroblasts and predicted by promoter analyses to target respiratory chain or mitochondrial genes (ChIP-Seq data). To test this hypothesis, KLF2 and ETV1 were independently silenced in NP fibroblasts and several mitochondrial parameters were tested. At the protein level, it was found that NRF1 and TFAM were restored to control levels following KLF2 or ETV1 silencing (Figure 25 A and B). More importantly, mitochondrial biogenesis was rescued in KLF2 and ETV1 knockdowns (Figure 25C), which ultimately resulted in increased mitochondrial function as assessed by real time respirometry (Figure 25D).

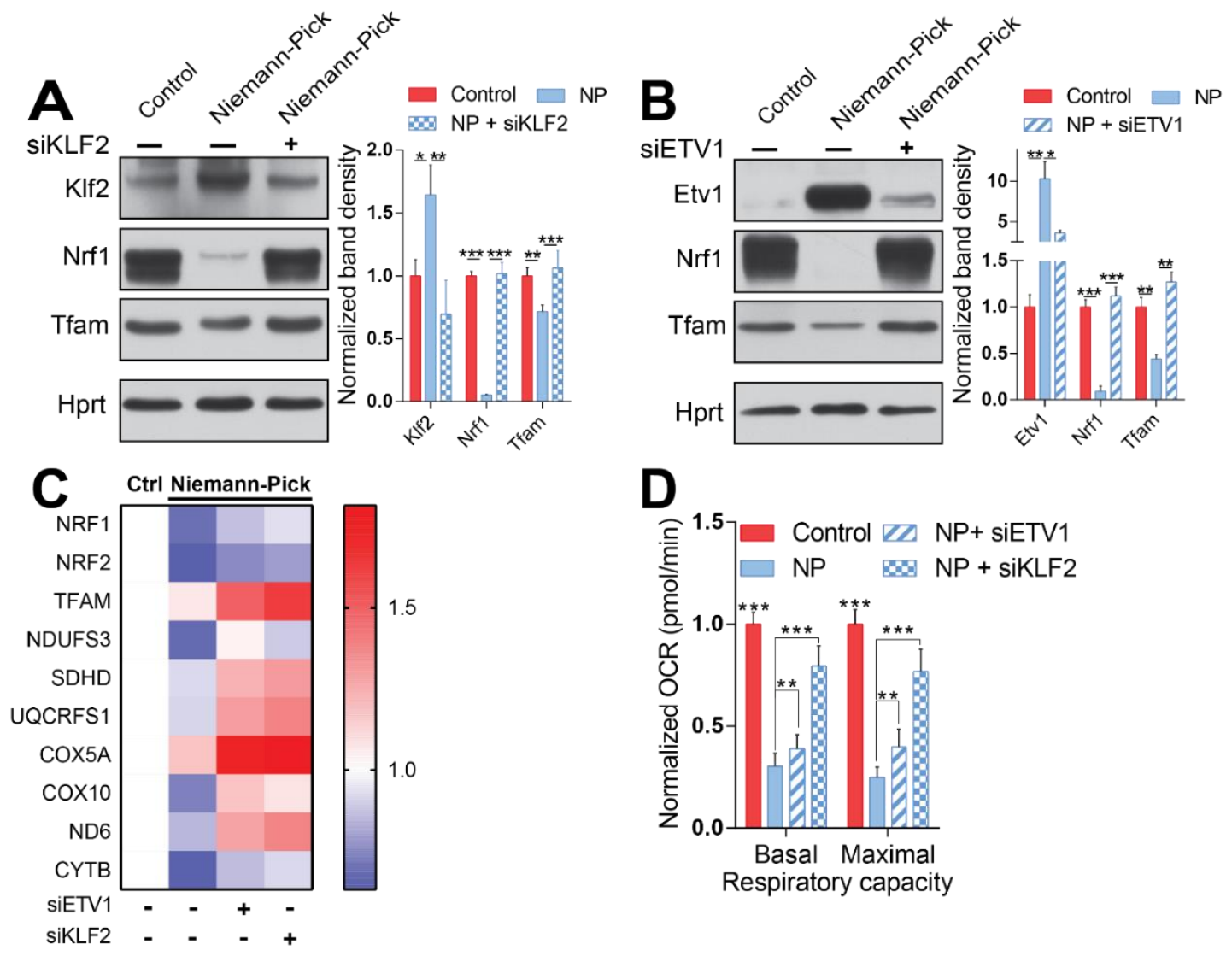

Figure 25| KLF2 and ETV1 repress mitochondrial biogenesis and function Silencing of KLF2 or ETV1 rescues NRF1 and TFAM levels (A and B), rescues mitochondrial biogenesis (C) and ultimately rescues mitochondrial respiration (D). Graphs depict mean \pm SEM for at least triplicate; $\mathrm{p}<0.05\left(^{*}\right), \mathrm{p}<0.01\left(^{* *}\right)$, and $\mathrm{p}<0.001\left({ }^{* *}\right)$; one-way ANOVA; SEM: standard error of the mean. Heatmap represents normalized mean plots of gene expression. 
Although silencing of either KLF2 or ETV1 improved mitochondrial outcomes in NP fibroblasts, it is noteworthy that this response was not due to alleviation of lysosomal stress. This evidence stems from the result that KLF2 or ETV1 knockdown had no effect on lysosomal malfunction as shown by persistent stalled autophagy in NP fibroblasts despite KLF2 or ETV1 silencing (Figure 26).
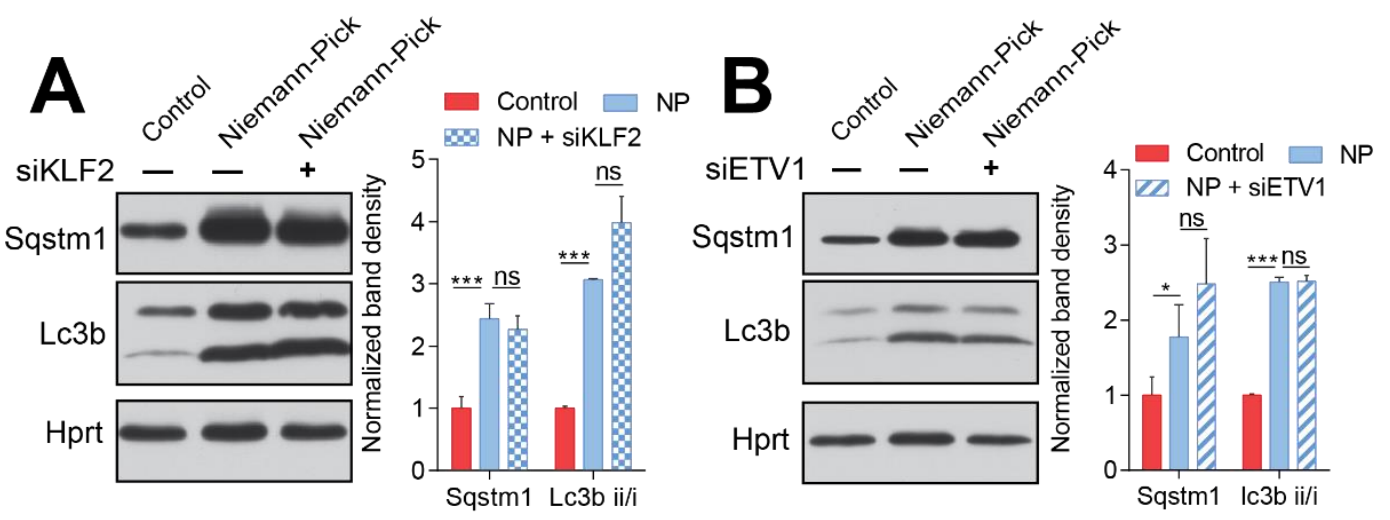

Figure 26| Autophagy defects in NP are independent of KLF2 and ETV1 - A and B Silencing of KLF2 or ETV1 does not alter autophagy defects in NP fibroblasts. Band quantifications are depicted as mean \pm SD for triplicate samples; $\mathrm{p}<0.05(*), \mathrm{p}<0.001\left(^{* * *}\right)$, and p > 0.05 (ns); one-way ANOVA; SD: standard deviation, ns: not statistically significant

\subsubsection{Mechanism of KLF2 and ETV1 regulation in Niemann-Pick disease}

Having shown that KLF2 and ETV1 mediate the repression of mitochondrial biogenesis in Niemann-Pick disease, we sought to elucidate how these two TFs were induced in the first place. Given that independent silencing of either KLF2 or ETV1 was enough to restore mitochondrial biogenesis in NP fibroblasts, it was rational to think that they may be cooperating in an epistatic fashion. Accordingly, it was observed that while KLF2 silencing also ablated ETV1 levels, ETV1 silencing had no noticeable effect on KLF2 levels (Figure 27A) implying that KLF2 regulated ETV1 activity. In addition, several reports have shown that the PI3K-AKT signaling pathway negatively regulates KLF2 expression (Sinclair et al., 2008; Skon et al., 2013) and interestingly, reduced AKT activity was observed in NP fibroblasts (Figure 27B). Furthermore, ETV1 has been known for a long time to be regulated by ERK signaling (Janknecht, 1996), whose activity was found to be increased in NP fibroblasts (Figure 27B). Recently, mTORC1 was found to respond to lysosomal cholesterol (Castellano et al., 2017), 
which accumulates in Niemann-Pick disease, and accordingly, increased mTORC1 activity was also observed, which is independent of its upstream regulator AMPK, the activity of which is not affected in NP fibroblasts (Figure 27B).
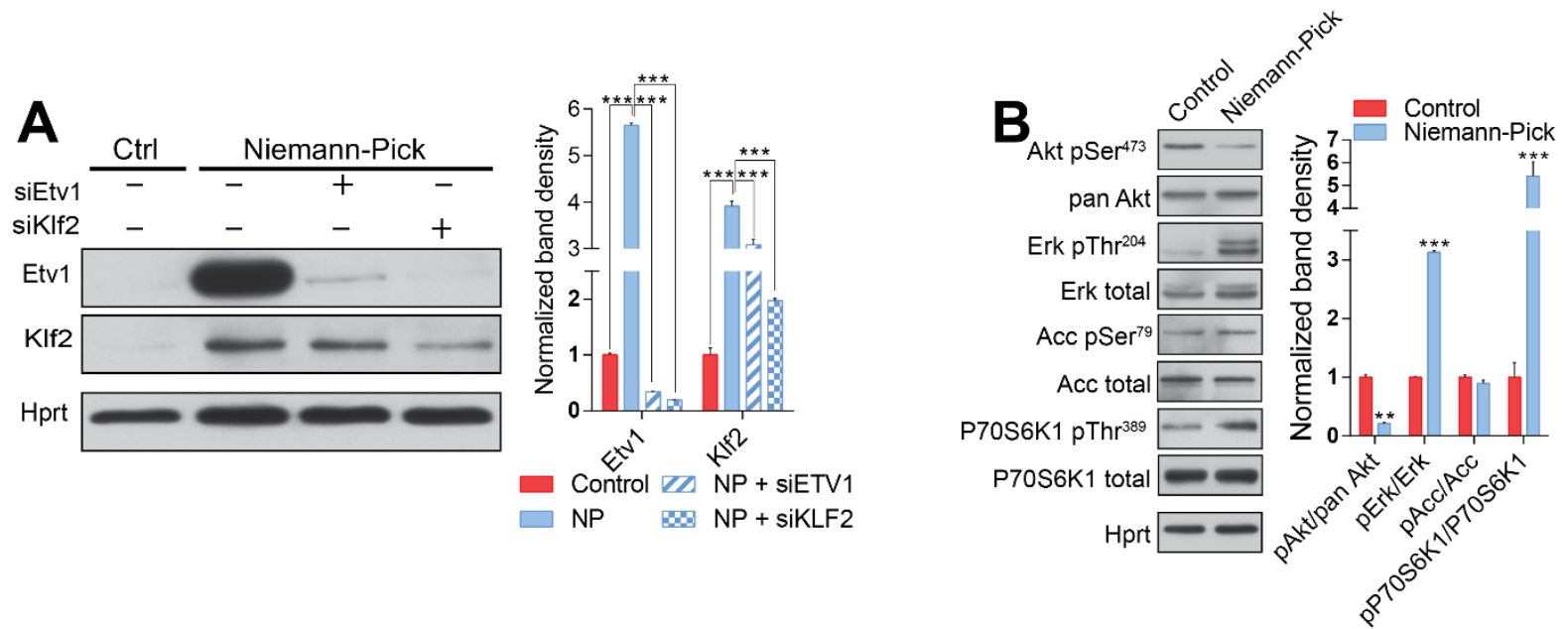

Figure 27| Mechanism of KLF2 and ETV1 induction - A KLF2 and ETV1 show epistatic regulation. Band quantifications are depicted as mean \pm Sd for triplicate samples and differences between means as based on one-way ANOVA tests with thresholds of $\left.\mathrm{p}<0.001{ }^{* * *}\right)$ were considered significant B NP fibroblasts retain only about $20 \%$ of AKT activity while inducing by 3- and 6-fold the activities of ERK and mTORC1 respectively. AMPK activity is not affected in NP fibroblasts. Graph in B represents mean $\pm \mathrm{SD}, \mathrm{N}=3 ; \mathrm{p}<0.01\left(^{* *}\right)$ and $\mathrm{p}<0.001\left(^{* * *}\right.$ ); Student's ttest; SD: standard deviation.

\subsubsection{KLF2 activates ETV1 in an ERK-dependent manner}

Since ERK levels, a positive regulator of ETV1, were found to be reduced in KLF2 hemizygous mice (Das et al., 2012), we reasoned that ERK signaling might also be mediating ETV1 induction by KLF2. To address this, NP fibroblasts were treated with the ERK inhibitor, U0126. Interestingly, pharmacological inhibition of ERK ablated ETV1 levels, although KLF2 levels were not affected in NP fibroblasts (Figure 28) suggesting that KLF2 induced ETV1 via an ERKdependent pathway. 


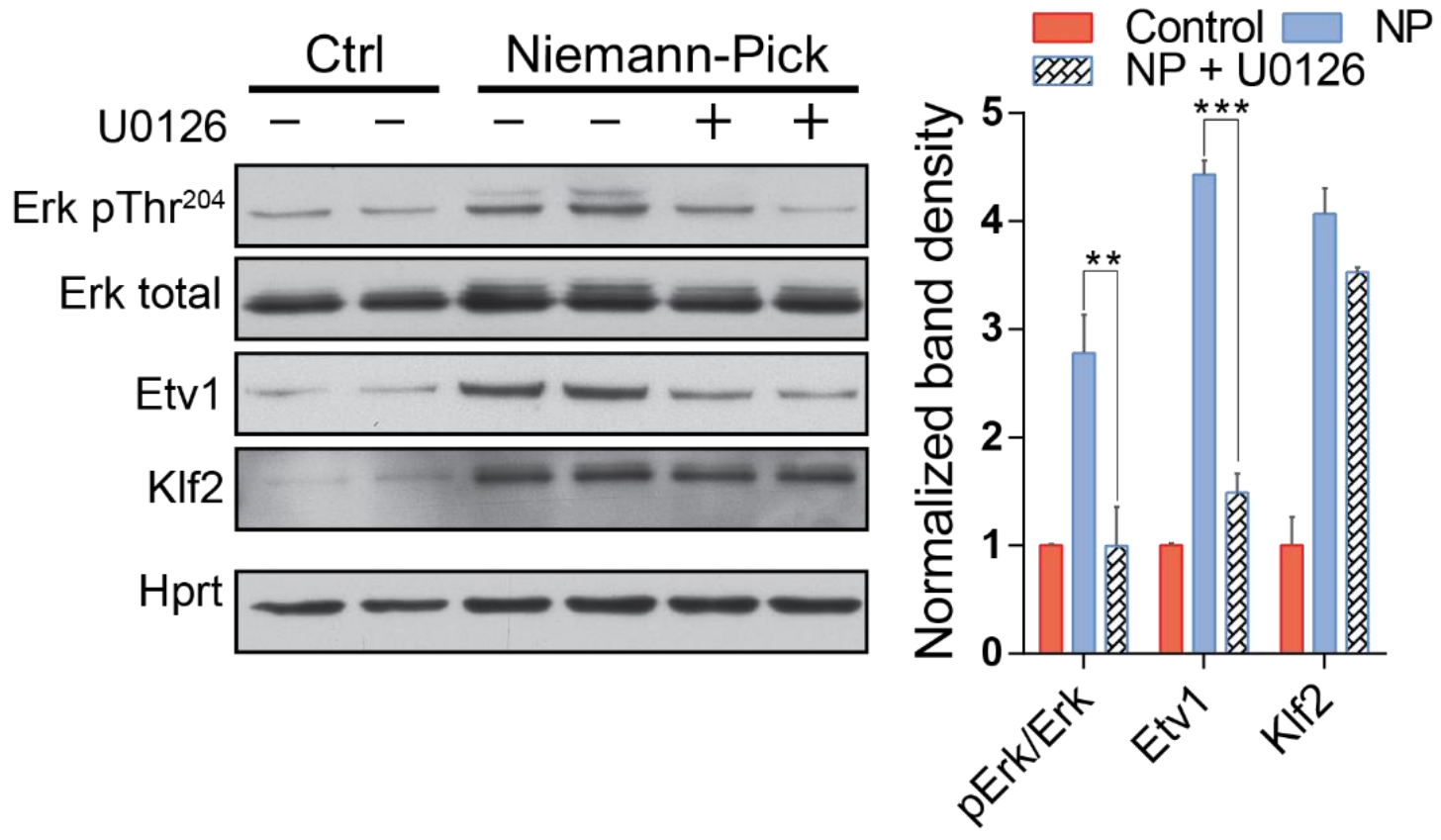

Figure 28| ETV1 induction is ERK dependent - Pharmacological inhibition of ERK activity

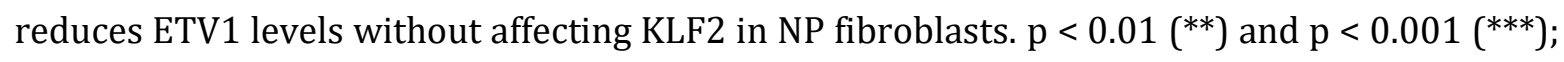
Student's test; mean \pm SD, $N=3$; SD: standard deviation.

\subsubsection{Impaired sphingosine-1-phosphate signaling activates KLF2}

Thus far, the mechanism driving ETV1 activity in Niemann-Pick disease was demonstrated. It remained however unclear how KLF2 was induced, even though reduced AKT activity in NP fibroblasts (Figure 27B) might be relevant for any mechanism thereof. Indeed, KLF2 drives the expression of S1PR1, which triggers a negative feedback loop targeting KLF2 for degradation via an AKT-dependent pathway (Bai et al., 2007; Sinclair et al., 2008; Skon et al., 2013). Given that the agonist for S1PR1 signaling, sphingosine-1-phosphate, is reduced in the plasma of Niemann-Pick disease patients (Fan et al., 2013), it was reasoned that KLF2 induction might be dependent on reduced S1PR1 signaling in Niemann Pick disease to induce the expression of S1PR1 as illustrated in Figure 29A. Although reduced AKT activity in NP fibroblasts may underlie reduced S1PR1 signaling, S1PR1 expression (a downstream target of KLF2 activity) was increased in Niemann-Pick disease (Figure 29B). Conversely, activation of S1PR1 signaling should reduce KLF2 levels and lower S1PR1 expression. Accordingly, control fibroblasts were treated with Sew2871, a potent and specific synthetic agonist of S1PR1 and, in agreement with our hypothesis, increased S1PR1 signaling resulted in decreased KLF2 and S1PR1 levels (Figure 29C). Having found reduced KLF2 levels following activation of S1PR1 
signaling, several mitochondrial parameters were tested as well. Importantly, increased levels of mitochondrial proteins were found, without any observable effects on lysosomal function as assessed by autophagy flux (Figure 29C). Mitochondrial gene expression was also increased in Sew2871-treated cells (see Appendix Figure 54). Altogether, these results support the notion that impaired sphingosine-1-phosphate signaling in Niemann-Pick disease activates KLF2, which represses mitochondrial biogenesis via ERK-dependent ETV1 induction. This pathway is independent of defective autophagy, implying that LSDs affect mitochondrial function via different mechanisms.

A

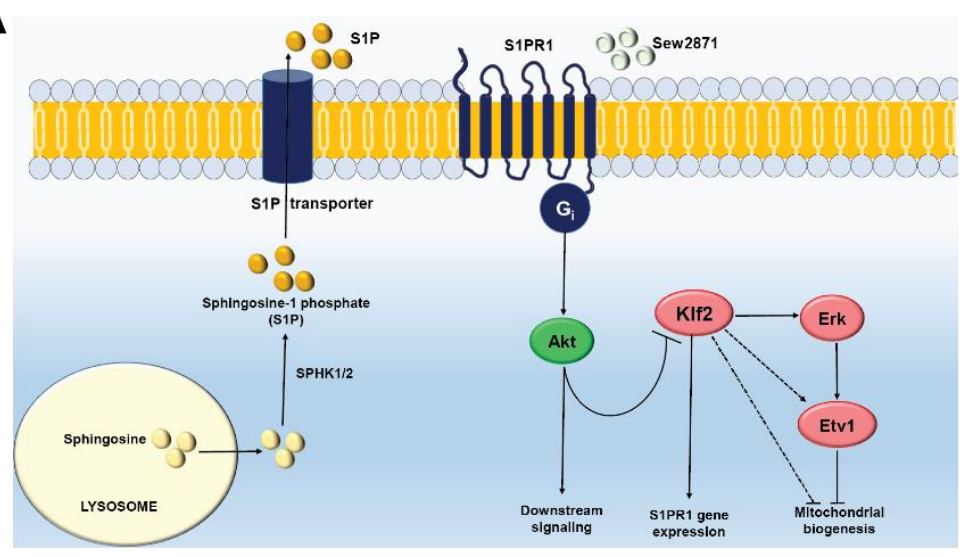

C

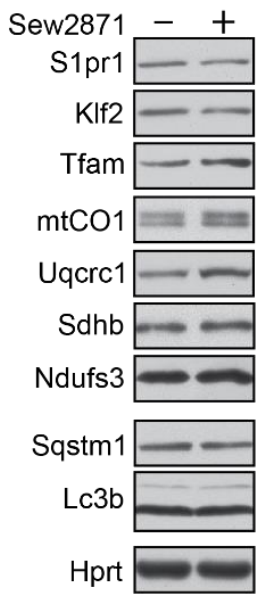

B
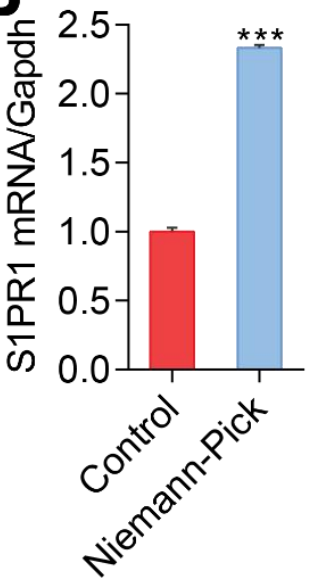

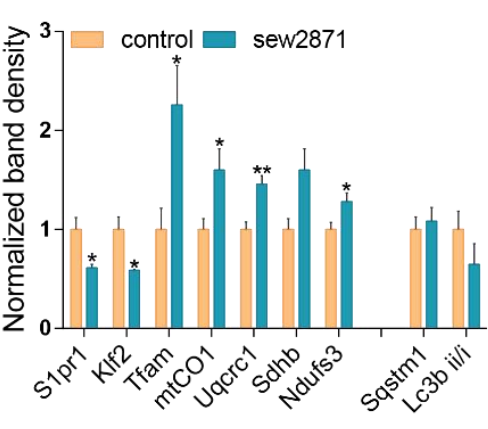

Figure 29| Impaired S1P signaling in NP - A Illustration of the S1PR1 signaling pathway.

B S1PR1 transcript levels are increased in NP fibroblasts. p <0.0001 ( ${ }^{* * *}$ ); Student's t-test; mean \pm SEM, N = 8 C Induction of S1PR1 signaling lowers KLF2 levels and promotes mitochondrial biogenesis. $\mathrm{p}<0.05\left(^{*}\right)$ and $\mathrm{p}<0.01\left(^{* *}\right)$; Student's t-test; mean $\pm \mathrm{SD}, \mathrm{N}=3$. SD: standard deviation, SEM: standard error of the mean 


\subsubsection{Activation of sphingosine 1-phosphate signaling restores mitochondrial}

\section{function in desipramine-treated cells}

We had shown previously that pharmacologic inhibition of ASM in control fibroblasts fully replicates mitochondrial deficits in NP fibroblasts with increased KLF2 and ETV1 levels (see section 3.1.5 and Appendix Figure 53). Having also shown that S1P signaling promotes mitochondrial biogenesis, we reasoned that activating S1P signaling in desipramine-treated cells should rescue mitochondrial function in these cells. Accordingly, we treated ASM-inhibited control fibroblasts with or without Sew2871 and assessed mitochondrial function by real time respirometry. Interestingly, while desipramine alone lowered mitochondrial OCR, coupling ASM inhibition with Sew2871 treatment resulted in a partial rescue of both basal and maximal mitochondrial respiratory capacity (Figure 30 ).

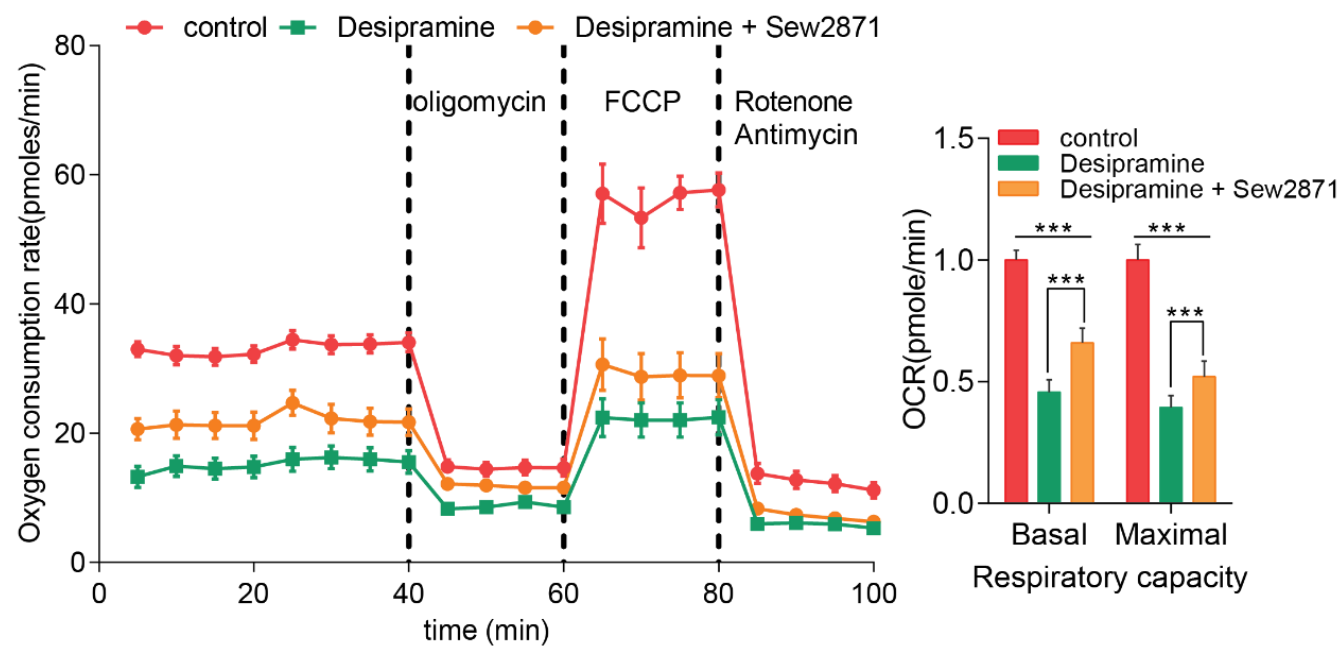

Figure 30| S1PR1 signaling rescues mitochondrial function in ASM inhibited cells

- Activation of S1PR1 signaling by Sew2871 treatment partially rescues basal and maximal mitochondrial OCR in desipramine-treated fibroblasts. Graphs represent mean \pm SEM, N = 8 differences between means as based on one-way ANOVA tests with thresholds of $\left.p<0.001{ }^{* * *}\right)$ were considered significant. SEM: standard error of the mean.

\subsubsection{Induction of KLF2 and ETV1 in Niemann-Pick disease is cytoprotective} Mitochondrial dysfunction is typically associated with the induction of apoptosis. As a result, it is reasonable to think that improving mitochondrial biogenesis and function via KLF2 and ETV1 downregulation in Niemann-Pick disease may be cytoprotective. On the other hand, the antiapoptotic role of KLF2 and ETV1 has been known for some time now given their induction in 
cancers (Alberts-Grill et al., 2016; Chi et al., 2010; Ohguchi et al., 2016). As a result, it was unclear to us whether the induction of KLF2 and ETV1 in NP fibroblasts was beneficial or detrimental to the cell. To clarify this, KLF2 and ETV1 were downregulated independently in NP fibroblasts and their impact on apoptosis was assessed by the Annexin V-Propidium iodide assay. While only about background proportion of apoptotic cells was observed in control cells, NP fibroblasts had about 10 -fold increase in the proportion of apoptotic cells relative to controls. This was further increased by 1.4- and 1.5-folds respectively following KLF2 and ETV1 silencing in NP fibroblasts (Figure $31 \mathrm{~A}-\mathrm{E}$ ). Taken together, these results support the finding that KLF2 and ETV1 exert their anti-apoptotic influence in NP fibroblasts with a consequent repression of mitochondrial biogenesis
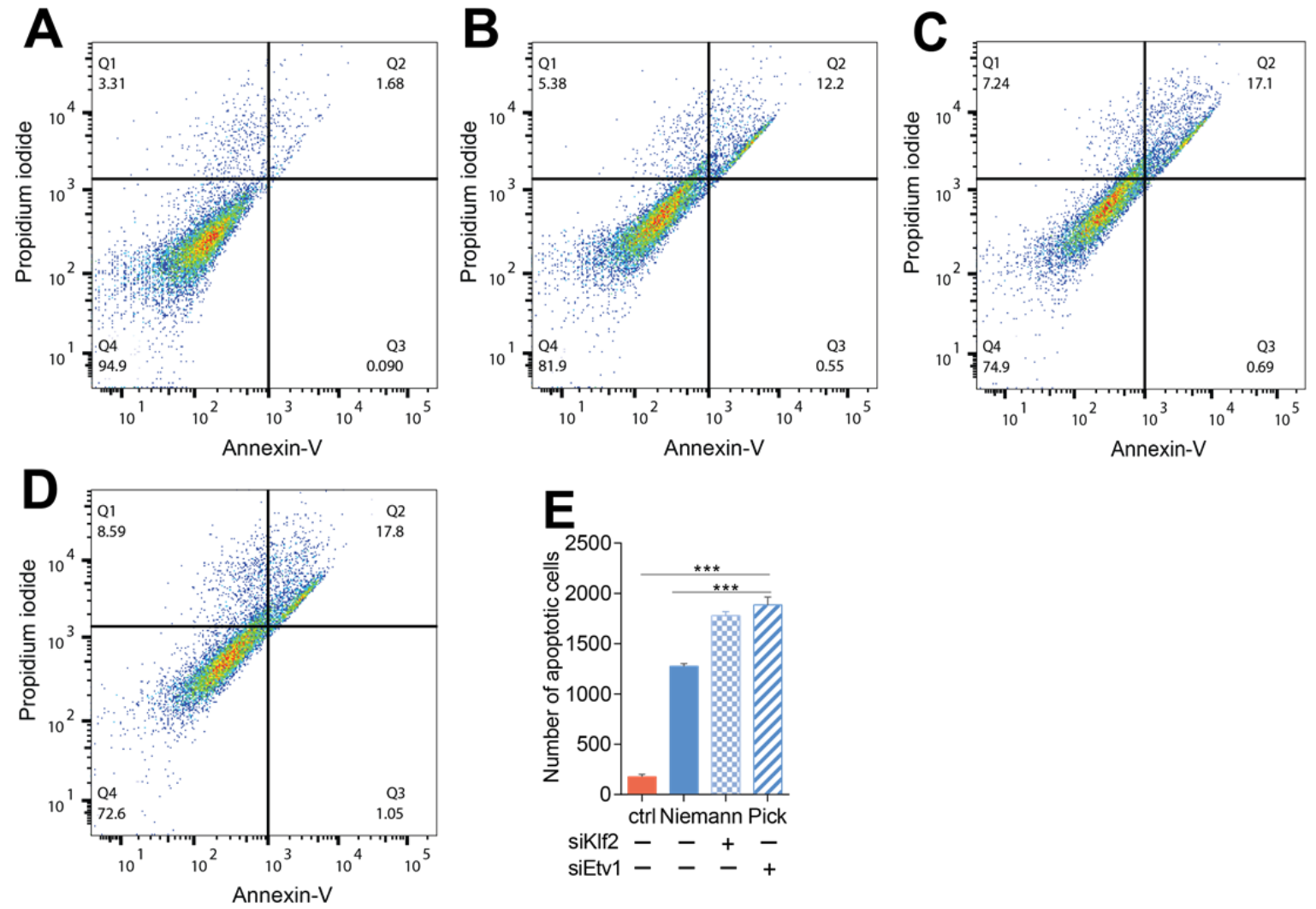

Figure 31| Induction of KLF2 and ETV1 is anti-apoptotic - Proportion of apoptotic cells in control (A) and NP (B) fibroblasts, and in NP fibroblasts following KLF2 (C) and ETV1 (D) silencing. E Graphical depiction of the increase in apoptosis in NP fibroblasts with/without KLF2 and ETV1 knockdown. $\mathrm{p}<0.001\left(^{* * *}\right)$; one-way ANOVA; mean $\pm \mathrm{SD}, \mathrm{N}=5$; SD: standard deviation 


\subsection{Impaired iron homeostasis due to lysosomal malfunction cause} mtDNA depletion, which induces TLR9-mediated inflammatory signaling.

\subsubsection{Cortical mitochondrial defects in Pompe's disease model}

The different types of Niemann-Pick disease form a spectrum of sphingolipid lysosomal storage disorders. The results from this study (section 3.1) and reports of additional studies demonstrate that the mechanism(s) underlying the pathogenesis of these diseases in part depend on impaired sphingolipid metabolism (Lachmann et al., 2004; Lloyd-Evans et al., 2008; Te Vruchte et al., 2004). It seems that mitochondrial dysfunction is a common feature of several LSDs and neurodegenerative diseases. However, not all LSDs primarily affect sphingolipid metabolism, suggesting that different mechanisms may underlie mitochondrial dysfunction in different LSDs. To elucidate some of these mechanisms, we focused on another lysosomal disease that affects glycogen storage, Pompe disease, which results from mutations in the acid alpha-glucosidase gene, GAA. The GAA protein is an enzyme that catalyzes the reaction of glycogen breakdown to glucose as illustrated in Figure 32A. We employed the GAA ${ }^{-1-}$ mice, an established mouse model of Pompe's disease.

Mitochondrial malfunction was reported in $\mathrm{GAA}^{-/}$mouse only recently, uncovering altered ultrastructural features and dynamics as well as reduced mitochondrial potential (Lim et al., 2015). We have further found evidence pointing to enhanced glycolysis, a common consequence of mitochondrial dysfunction, in $\mathrm{GAA}^{-/}$MEFs and HeLa cells with shRNAmediated silencing of GAA (Figure 40 and Appendix Figure 55). Pompe's disease literature is dominated by studies in cardiac and skeletal muscles, since they are the most affected tissues. Nervous system involvement in the Pompe's disease mechanism has only been recently reported in the peripheral nervous system (Falk et al., 2015). It was however unclear whether central nervous system (CNS) defects were involved in Pompe's disease. As a result, we sought to find out if, as in Niemann-Pick disease (as shown before), a transcriptional network of mitochondrial biogenesis and function was altered in Pompe's disease. Mitochondrial biogenesis was therefore assessed in the cortex of $\mathrm{GAA}^{--}$mice by measuring the expression of relevant transcriptional regulators of mitochondrial biogenesis and function. 


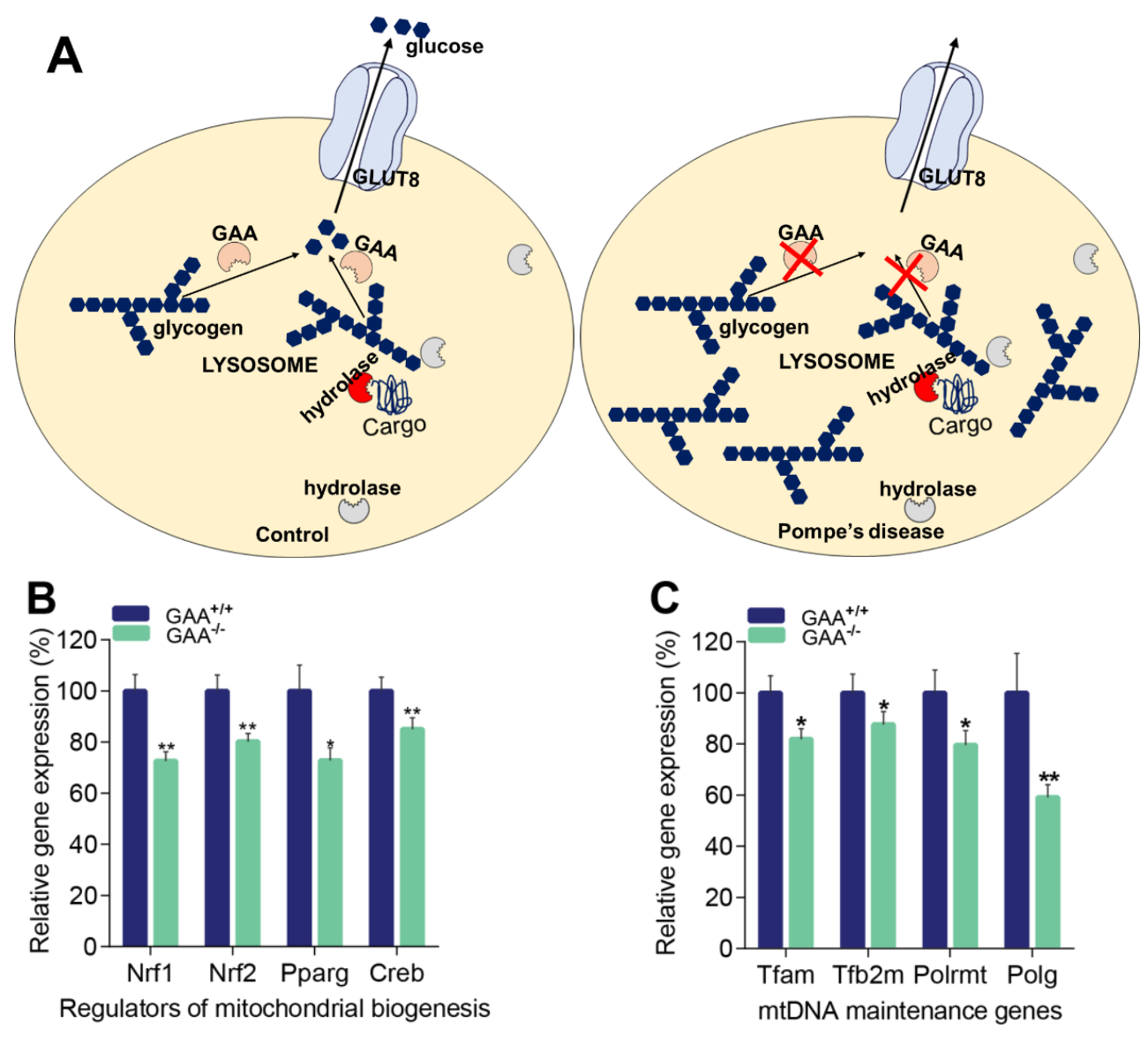

Figure 32| Repressed mitochondrial biogenesis in $\mathbf{G A A}^{-/}$cortex - A Illustration of the activity of GAA in healthy (left) and Pompe's disease lysosomes (right). Lack of GAA activity results in intralysosomal glycogen accumulation in Pompe's disease B Transcript levels of mitochondrial biogenesis mediators are reduced in the cortex of $\mathrm{GAA}^{\%}$ mice. $\mathbf{C}$ mtDNA transcriptional activity is downregulated following reduced expression of mtDNA maintenance genes in $\mathrm{GAA}^{-/}$mice Gene expression is depicted as mean \pm SEM, N $=8$; $\mathrm{p}<0.05\left(^{*}\right)$ and $\mathrm{p}<0.01$ ${ }^{* *}$; Student's t-test; SEM: standard error of the mean

The expression of NRF1, NRF2, PPARG, and CREB, which are known transcriptional regulators of nuclear-encoded mitochondrial genes, was decreased (Figure 32B). In addition, essential factors that regulate the replication and transcription of mtDNA: TFAM, TFB2M, POLRMT, and POLG were all reduced in the cortex of $\mathrm{GAA}^{-/}$mice (Figure 32C). Accordingly, reduced NRF1, NRF2, and TFAM protein levels were also observed in the GAA ${ }^{-/}$mice (Figure 33). 


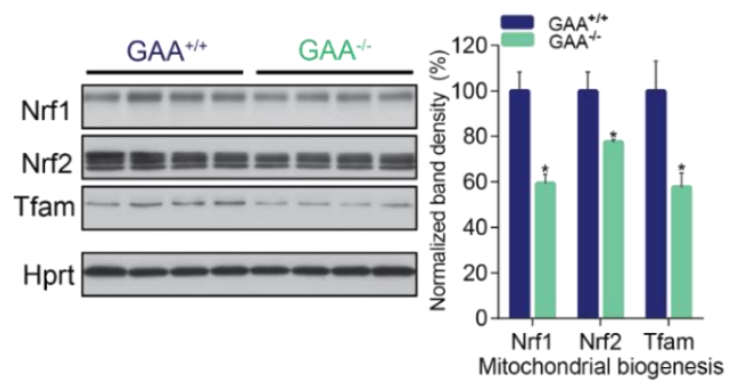

Figure 33| Reduced transcriptional regulation of mitochondrial biogenesis Mitochondrial biogenesis requires cooperation between two genomes, whose key regulator proteins, NRF1, NRF2 and TFAM are less abundant in the cortex of $\mathrm{GAA}^{\%}$ mice. Band quantifications are represented as mean \pm SEM, $N=8$; $<0.05\left(^{*}\right)$; Student's t-test; SEM: standard error of the mean.

\subsubsection{Progressive cortical mtDNA depletion in $\mathrm{GAA}^{-/-}$mice}

Maintenance of the mitochondrial genome, as well as its transcription and translation, is controlled by proteins and enzymes which are encoded in the nucleus (reviewed in Gustafsson et al., 2016). Given that reduced amounts of these factors were found in the cortex of GAA ${ }^{-1-}$ mice, it was reasoned that mtDNA depletion would likely occur in the brain. To this end, total DNA was isolated from cortices of $\mathrm{GAA}^{-/}$mice and their wild-type littermates at different age points and mtDNA copy numbers were measured (relative to a nuclear DNA control). Interestingly, progressive decline in mtDNA copy numbers was found in $\mathrm{GAA}^{-/}$mice culminating in a $60 \%$ loss at 6 months of age (Figure 34).

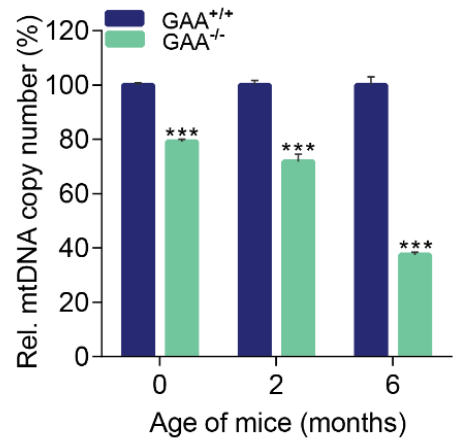

Figure 34| Progressive mtDNA copy number depletion in GAA $/$ - cortex - mtDNA copy numbers at 0, 2 and 6 months of age in wild-type and GAA $\%$ mice cortices show progressive mtDNA depletion with age. $\left.\mathrm{p}<0.001{ }^{* * *}\right)$; Student's t-test; mean $\pm \mathrm{SEM}, \mathrm{N}=8$. 


\subsubsection{Cardiac mitochondrial deficits in Pompe's disease}

Having established that a decreased transcriptional network of mitochondrial biogenesis and function ensued in the CNS of GAA ${ }^{-/}$mice, it was relevant to determine whether a similar response occurred in the heart of $\mathrm{GAA}^{-/-}$mice since cardiomyopathy is a reported phenotype of Pompe's disease. Similarly, mtDNA copy numbers were quantified from total DNA isolated from the heart of $\mathrm{GAA}^{-/}$mice and their control littermates at 2 and 6 months of age. Notably, a decline in mtDNA copy numbers was also observed in the heart, albeit not progressive as in the CNS, since mtDNA was depleted at 2- and 6-months of age to similar levels (Figure 35).

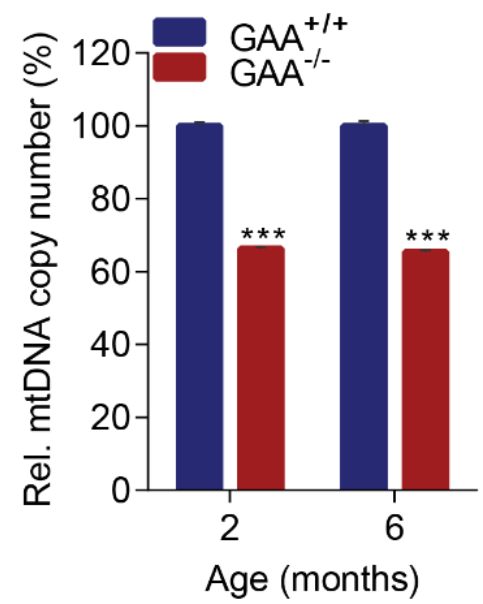

Figure 35| Cardiac mtDNA copy number declines in GAA loss - Although not progressive with age, mtDNA copy number depletion is evident in the heart of GAA $\%$ mice. $\mathrm{p}<$ 0.001 ( $^{* * *}$; Student's t-test; mean \pm SEM, $N=8$; SEM: standard error of the mean

We hypothesized that a transcriptional component may mediate mtDNA depletion in the heart. To verify this, transcript and protein levels of the key regulators of mitochondrial biogenesis and function were measured in the heart of $\mathrm{GAA}^{-/}$mice. Surprisingly, at transcript level, the expression of NRF1, NRF2, PPARG, and CREB were markedly increased (Figure 36A). The increase in the expression level of genes that regulate mtDNA maintenance (Figure 36B) was even more robust. The protein levels of these regulators were not changed consistently, as we observed $60 \%$ increase in NRF1 levels, unchanged NRF2 levels and a $50 \%$ decrease in TFAM levels (Figure $36 \mathrm{C}$ ). Taken together, the results from the cortex and heart of $\mathrm{GAA}^{-/}$mice implied that mtDNA depletion was a common phenotype associated with Pompe's disease and that decline in mtDNA copy numbers was independent of mitochondrial biogenesis. TFAM is known to regulate mtDNA transcription and replication, and TFAM haploinsufficiency and 
overexpression studies suggest that levels of TFAM correlate well with mtDNA copy number levels (West et al., 2015; Xu et al., 2009). It has also been shown, conversely, that inhibiting mIDNA replication by ethidium bromide treatment results in TFAM degradation without altering TFAM mRNA levels (Matsushima et al., 2010). Thus, it remained unclear to us whether mtDNA depletion was due to reduced TFAM levels, or whether reduced TFAM levels were a consequence of mtDNA depletion. Interestingly, reduced mtDNA copy numbers and TFAM levels were also observed in Niemann-Pick disease (Figures 14C and 15). That notwithstanding, it was important to understand the mechanism(s) directing mtDNA depletion since its copy number variation has been linked to aging and several neurodegenerative diseases including AD, PD and ALS (reviewed in Keogh and Chinnery, 2015; Pinto and Moraes, 2014).
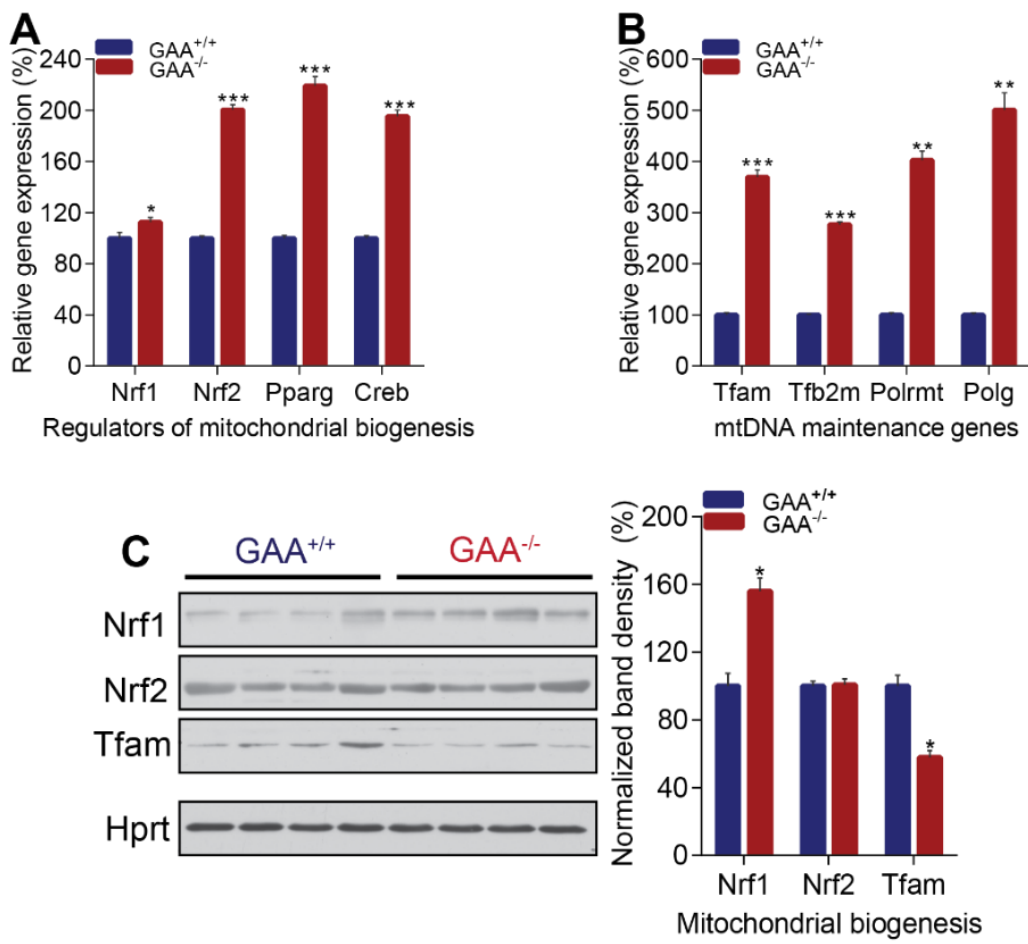

Figure 36| Increased cardiac mitochondrial biogenesis in GAA ${ }^{-/}$mice A Transcriptional regulation of mitochondrial biogenesis is increased in the heart. B Cardiac mtDNA transcriptional activity is induced in GAA $\%$. C Western blot analyses of heart lysates show increased NRF1 levels and reduced TFAM protein amounts. NRF2 protein amounts are not altered in $\mathrm{GAA}^{-/}$mice. Gene expression and band quantifications are displayed as mean $\pm \mathrm{SEM}$ for $\mathrm{N}=8 ; \mathrm{p}<0.05\left(^{*}\right), \mathrm{p}<0.01\left(^{* *}\right)$, and $\mathrm{p}<0.001\left(^{* * *}\right)$; Student's tests; SEM: standard error 


\subsubsection{Loss of TFAM but not mtDNA depletion in $\mathrm{GAA}^{-/ /}$is dependent on mTORC1}

In order to mechanistically probe mtDNA depletion in lysosomal malfunction, it was necessary to employ a model that is amenable to experimental manipulation. As such, it was tested if $\mathrm{GAA}^{--}$MEFs would replicate the mtDNA defect that was observed in lysosomal malfunction models. Figure 38B showed a reduction in mtDNA copy number levels in $\mathrm{GAA}^{-1-} \mathrm{MEFs}$. It was then tested whether TFAM induction would rescue mtDNA copy numbers. Given that the transcript levels of TFAM were only slightly reduced in the brain and increased by fourfold in the heart, it was reasoned that enhancing TFAM translation would be a better mechanism of TFAM induction. The mechanistic/mammalian target of rapamycin complex 1 (mTORC1) was recently shown to specifically direct mitochondrial biogenesis through 4E-BP-dependent translational regulation of TFAM and other mitochondrial-related mRNAs (Morita et al., 2013). Thus, mTORC1 activity, which was found to be reduced in the cortex (Appendix Figure 56) and MEFs, was increased by siRNA-mediated TSC2 silencing in GAA ${ }^{-/}$MEFs (Figure 37).

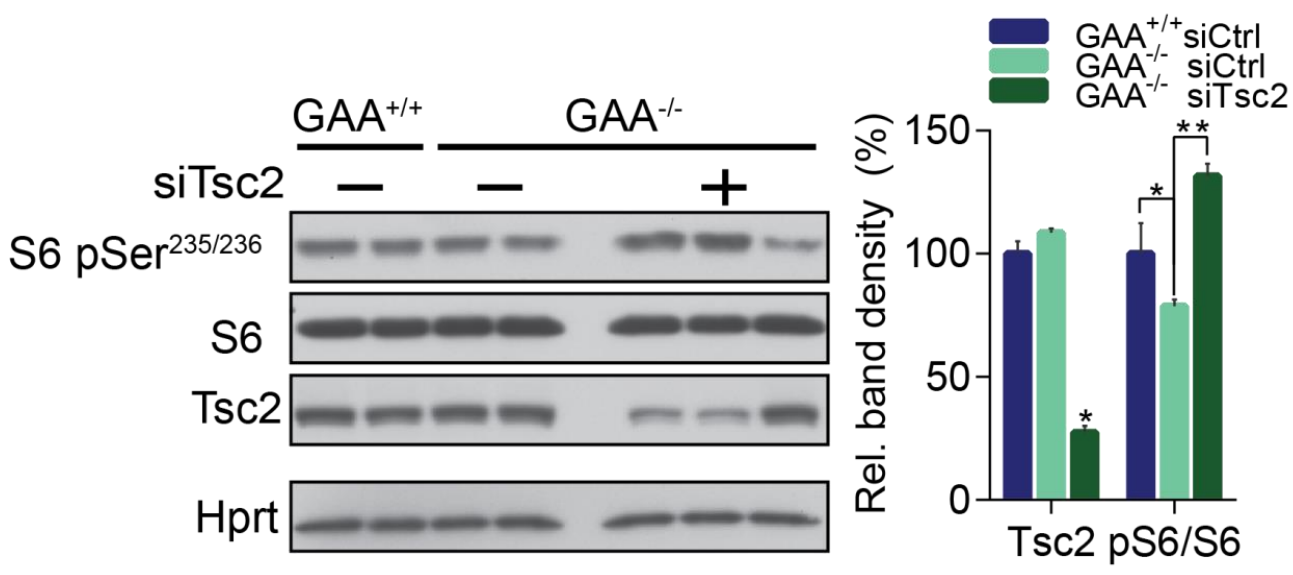

Figure 37| Induction of mTORC1 activity in GAA $\%$ MEFs - siRNA-mediated silencing of Tuberin (TSC2), which negatively regulates mTORC1 activity, results in increased mTORC1 activity as demonstrated by phosphorylation of ribosomal protein S6 at Serine 235/236. Band quantifications are depicted as mean \pm SD for triplicate samples; $\mathrm{p}<0.05\left(^{*}\right)$ and $\mathrm{p}<0.01\left({ }^{* *}\right)$; one-way ANOVA; SD: standard deviation 
TSC2 silencing, as expected, resulted in increased mTORC1 activity as assessed by the phosphorylation of Serine residues 235/236 of the ribosomal protein s6 (Figure 37), which led to a robust rescue of TFAM protein amounts to control levels in the GAA $^{-/}$MEFs (Figure 38A). However, mtDNA copy number levels were affected neither by increased mTORC1 activity nor by rescued TFAM levels (Figure 38B). Therefore, this result implied that mtDNA depletion was not a consequence of reduced TFAM levels. The reverse, however, may be true given that increased mTORC1 activity in $\mathrm{GAA}^{-/}$MEFs also led to a slight but significant decrease in the protein levels of Lon protease, which degrades TFAM (Appendix Figure 57).
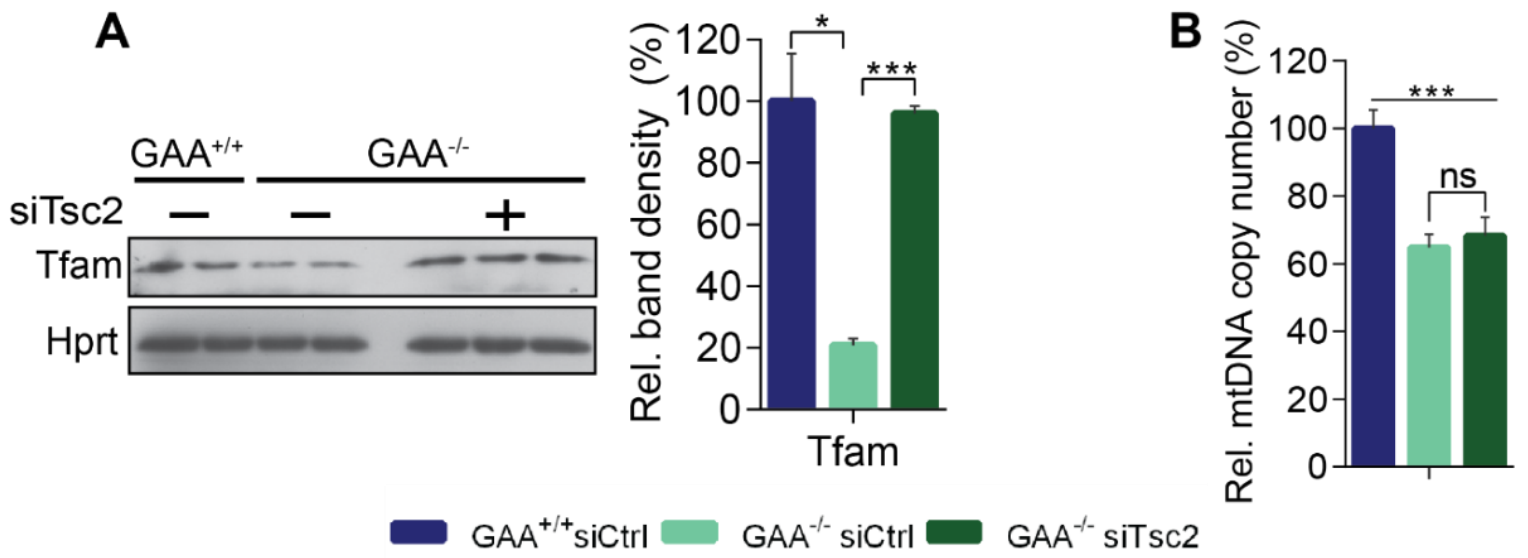

Figure 38| Increased mTORC1 activity reverses TFAM loss - A Increased mTORC1 activity rescues TFAM levels. Band quantifications are depicted as mean \pm SD for triplicate samples; $\mathrm{p}<0.05\left(^{*}\right)$ and $\mathrm{p}<0.001\left(^{* * *}\right)$; one-way ANOVA; SD: standard deviation B mtDNA depletion is unaffected by mTORC1 activity or TFAM levels in GAA $\%$ MEFs. Graph represents mean \pm SEM, N=8; p > 0.05 (ns) and $\mathrm{p}<0.001$ ( $^{* * *}$ ); one-way ANOVA; SEM: standard error of the mean, ns: not statistically significant. 


\subsubsection{Impaired nucleotide metabolism affects mtDNA levels}

Although several nuclear-encoded genes drive mtDNA synthesis and maintenance, a ratelimiting component of this reaction is the bioavailability of intra-mitochondrial deoxyribonucleotide triphosphates (dNTPs) - the pool of building blocks for mtDNA synthesis. Furthermore, reduced activity of enzymes and kinases, which regulate the bioavailability of dNTPs, is a recognized cause of mtDNA depletion syndromes (El-Hattab et al., 2017). Having demonstrated that increasing TFAM levels does not alter mtDNA copy number, it was reasoned that impaired nucleotide metabolism may be a culprit in mtDNA depletion in GAA insufficiency.

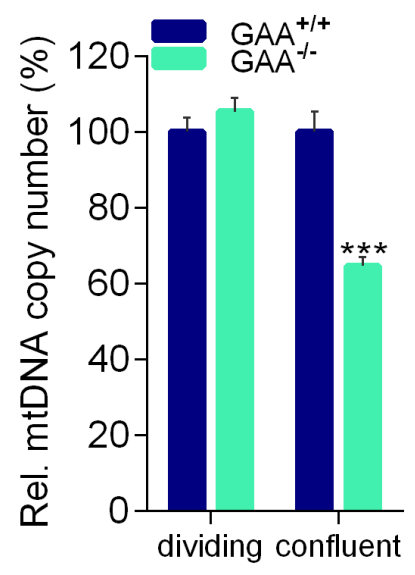

Figure 39| Nucleotide metabolite availability affects mtDNA levels - GAA $\%$ MEFs cultured under dividing and non-dividing conditions show distinct mtDNA copy number phenotypes with a decline only under confluent conditions. Graph represents mean \pm SEM, $\left.\mathrm{N}=8 ; \mathrm{p}<0.001{ }^{* * *}\right)$; one-way ANOVA; SEM: standard error of the mean.

To address this, GAA ${ }^{-1-}$ MEFs were cultured for 3 days in dividing and non-dividing conditions and mtDNA copy number was quantified. The reasoning behind this was that, during cell division, de novo biosynthesis of nucleotides is increased to make nucleotides available for DNA replication. These nucleotides also become bioavailable for mtDNA synthesis. On the other hand, under non-dividing conditions, mtDNA synthesis is markedly dependent on the salvage pathway for dNTPs - a mechanism, which is especially relevant for mtDNA maintenance in post-mitotic cells. Interestingly, it was found that while mtDNA copy number levels were unchanged during dividing conditions, there was a rapid decline in mtDNA copy number levels in GAA ${ }^{-1-}$ MEFs when the cells were confluent (Figure 39). 
This result was consistent with our idea of impaired nucleotide metabolism in GAA knockout. Interestingly, this result was supported by our data from metabolite profiling of HeLa cells with shRNA-mediated silencing of GAA (Figure 40). Nucleotide biosynthetic pathway metabolites were decreased while glycolysis intermediates were increased in shGAA cells (Figure 40).

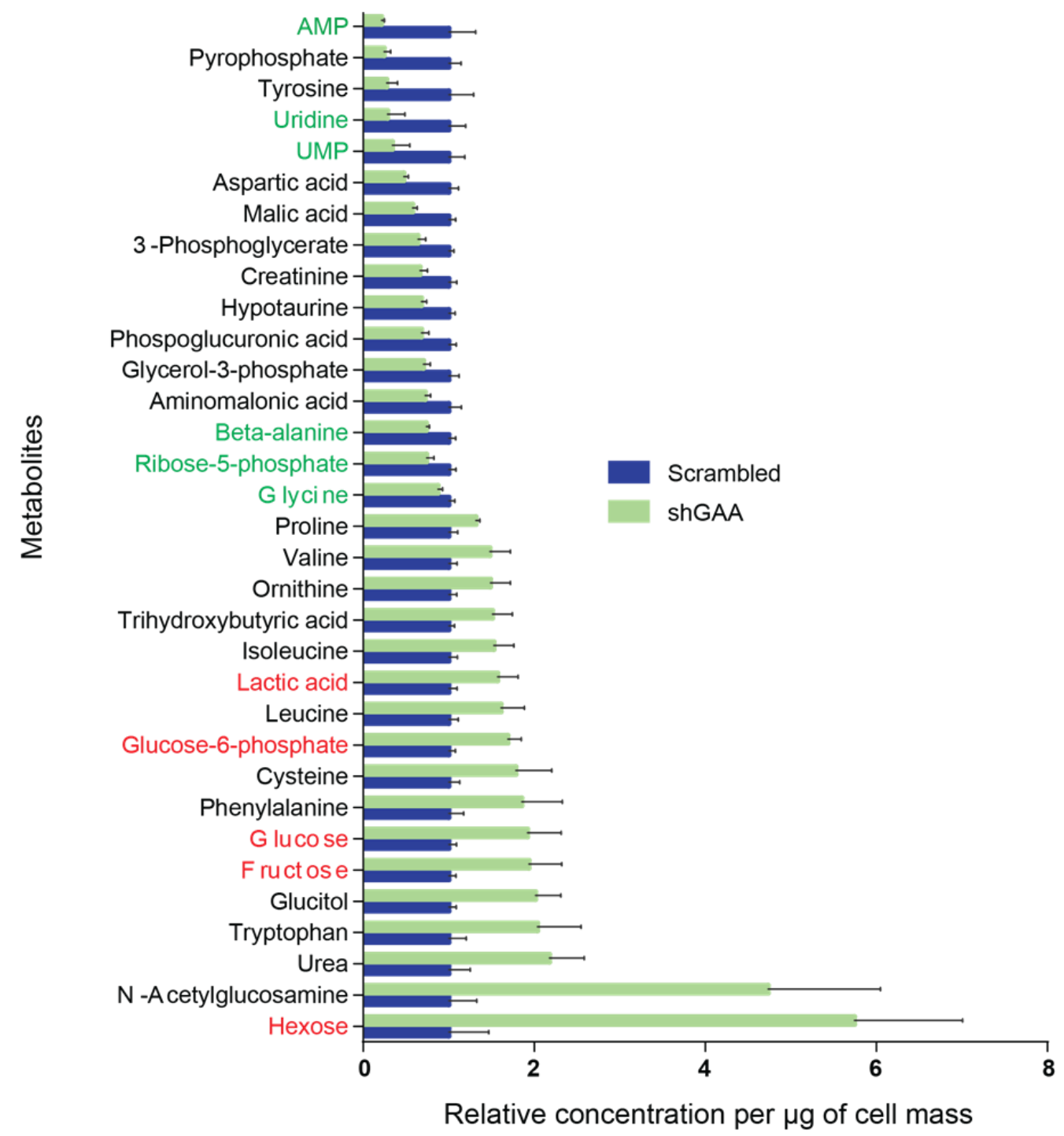

Figure 40| Metabolite profiling of shGAA HeLa cells - The graph above depicts metabolites with significantly altered concentrations following shRNA-mediated silencing of GAA in HeLa cells. Graph represents mean \pm SEM, $n=10$; $p<0.05$; Student's test; SEM: standard error of the mean. Glycolysis intermediates (in red) are increased while nucleotide biosynthetic components (in green) are reduced in shGAA cells. 
Table 16: Altered metabolic pathways in shGAA cells

\begin{tabular}{lllll}
\hline \hline pathway & p-value & total & expected & hits \\
\hline Pyrimidine metabolism & 0.001255 & 60 & 0.49855 & 4 \\
Glycolysis & 0.002370 & 21 & 0.61100 & 4 \\
Nitrogen metabolism & 0.003707 & 39 & 0.32405 & 3 \\
Galactose metabolism & 0.004278 & 41 & 0.34067 & 3 \\
Starch and sucrose metabolism & 0.007498 & 50 & 0.41545 & 3 \\
Thiamine metabolism & 0.016299 & 24 & 0.19942 & 2 \\
Purine metabolism & 0.038457 & 92 & 0.76444 & 3 \\
\hline \hline
\end{tabular}

In addition, metabolite set enrichment analyses of the altered metabolites revealed the metabolic pathways that were significantly affected in GAA knockdown cells (Table 16). It was interesting to find that nucleotide metabolism (in red) as well as glycolysis scored as significantly affected metabolic pathways in cells with GAA downregulation.

Further, it was reasoned that enhancing nucleotide metabolism should protect against mtDNA depletion. For this purpose, GAA wild type and knockout MEFs were treated with folic acid (or $\mathrm{NaOH}$ as vehicle control) that regulates nucleotide synthesis through one-carbon metabolism, (Nikkanen et al., 2016; Tufi et al., 2014) and mtDNA levels were measured. Notably, folate supplementation resulted in enhanced one-carbon metabolism by increasing levels of methylenetetrahydrofolate dehydrogenase 2 (MTHFD2) in GAA ${ }^{-1}$ MEFS (Figure 41A). MTHFD2 is an essential and rate-limiting enzyme of the mitochondrial folate metabolic pathway, which donates single carbon molecules for nucleotide synthesis (Di Pietro et al., 2004). Strikingly, mtDNA defects were rescued in $\mathrm{GAA}^{-/} \mathrm{MEFs}$ with folate supplementation (Figure 41B). Altogether, these results demonstrate that impaired nucleotide metabolism is part of the mechanism of mtDNA depletion upon GAA loss, and probably in other lysosomal storage diseases too. However, it was still unclear how lysosomal malfunction triggered by GAA insufficiency resulted in impaired nucleotide metabolism and, consequently, mtDNA copy number loss. 

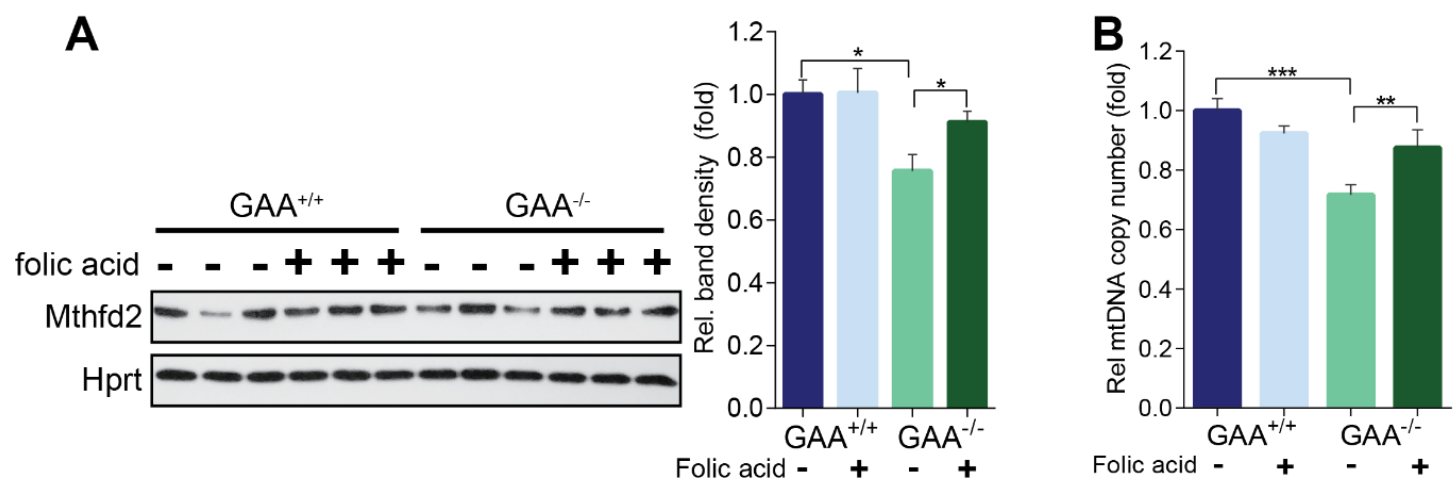

Figure 41| Enhancing nucleotide synthesis rescues mtDNA depletion - A Folic acid supplementation induces folate metabolism in $\mathrm{GAA}^{-}$- MEFs as shown by increased MTHFD2 levels. B Induction of folate metabolism via folic acid supplementation reverses mtDNA loss in $\mathrm{GAA}^{-/-}$MEFs. Graphs show mean \pm SEM of at least triplicate samples; $\mathrm{p}<0.05\left(^{*}\right), \mathrm{p}<0.01\left(^{* *}\right)$, and $\mathrm{p}<0.001\left(^{* * *}\right)$; one-way ANOVA; SEM: standard error of the mean

\subsubsection{Lysosomal malfunction impairs iron homeostasis}

Nucleic acids, like other macromolecules, are degraded continuously in cells. The products of such nucleic acid catabolism are either excreted or salvaged as components of new nucleotides. In fact, in quiescent cells, the salvage pathway of nucleic acid metabolism is of utmost relevance for mtDNA replication requiring the activity of ribonucleotide reductase subunit p53R2 (Pontarin et al., 2012) to make dNTPs available. However, no change in the levels of ribonucleotide reductase p53R2 was observed in $\mathrm{GAA}^{-/-}$brain or heart (see Appendix Figure 58). In addition, ribonucleotide reductase subunit p53R2 harbors an iron-tyrosyl free radical center, which is a required co-factor for initiation of nucleotide reduction (Bourdon et al., 2007; Guittet et al., 2001). Given that the endo-lysosomal system has been known for some time now to be central to iron homeostasis (reviewed in Biasiotto et al., 2016; Kurz et al., 2008; Rouault, 2013), it was thought that impaired iron homeostasis due to lysosomal malfunction might be responsible for reduced ribonucleotide reductase activity, impaired nucleotide metabolism and as a consequence, mtDNA instability and depletion. 

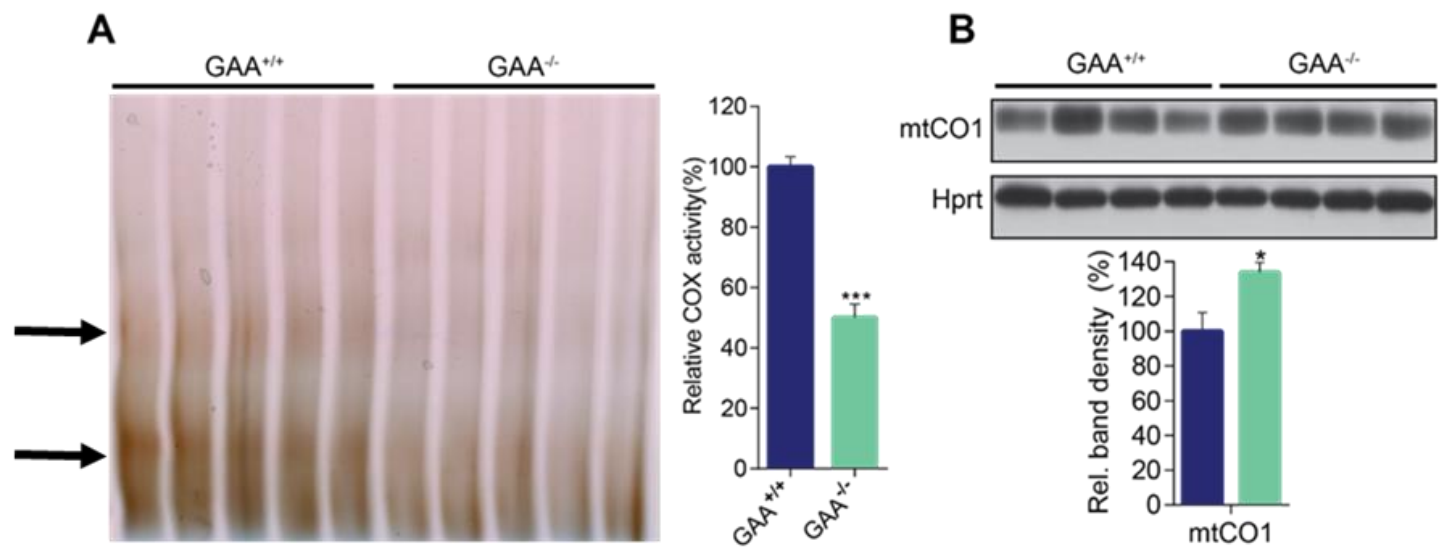

Figure 42| Decreased complex IV activity in GAA-/- cortical mitochondria - A Native PAGE and subsequent activity staining of mitochondria purified from GAA $\%$ mice show 50\% reduction in complex IV activity. Arrows indicate supercomplexes (top) and complex IV monomers (bottom). B Protein amounts of the mtDNA-encoded mitochondrial cytochrome c oxidase subunit 1 (mtCO1) are increased in the cortex of $\mathrm{GAA}^{\%}$ mice. Quantifications are displayed as mean \pm SEM for $N=8 ; \mathrm{p}<0.05\left(^{*}\right)$ and $\mathrm{p}<0.001\left(^{* * *}\right)$; Student's tests; SEM: standard error

Preliminary evidence of such impaired iron handling came from the result that isolated mitochondria from the cortex of $\mathrm{GAA}^{-/-}$mice, when subjected to BN-PAGE and subsequent activity staining showed about $50 \%$ reduction in complex IV activity relative to their littermate controls (Figure 42A). However, the steady-state levels of mitochondrial cytochrome c oxidase subunit 1 (mtCO1) were not reduced in the cortex of $\mathrm{GAA}^{-/-}$mice (Figure 42B). Since mtCO1 is the catalytic enzyme of complex IV of the respiratory chain and displays a steady state-linked catalytic activity (D'Aurelio et al., 2001; Varlamov et al., 2002) it was reasoned again that iron (a cofactor for complex IV activity) may be mishandled. To further demonstrate iron deficiency in $\mathrm{GAA}^{-/-}$mice, levels of transferrin receptor and ferritin were measured in the cortex of $\mathrm{GAA}^{-/-}$ mice. It is widely known that under conditions of iron deficiency, transferrin receptor levels are increased to enhance iron delivery into the cell through endocytosis, and that ferritin amounts are reduced to lower sequestration of iron and thus replenish cellular iron content (Rouault, 2013). Similarly, and consistent with an idea of iron deficiency in $\mathrm{GAA}^{-/}$mice, increased transferrin receptor levels and decreased ferritin amounts were observed in the cortex (Figure 43A). To confirm these results, iron concentration in the $\mathrm{GAA}^{-/-}$MEFs was measured using an iron assay kit (see Methods). Curiously, total iron concentration was not altered but levels of ferrous iron were significantly reduced in $\mathrm{GAA}^{-/-} \mathrm{MEFs}$, while those of ferric iron were increased 
(Figure 43B). The relevance of this result stemmed from its consistency with lysosomal malfunction: the lysosome harbors the metalloreductase STEAP3, which reduces $\mathrm{Fe}^{3+}$ to $\mathrm{Fe}^{2+}$. As such, the results reflect a functional iron pseudo-deficiency state, where $\mathrm{Fe}^{2+}$ levels are reduced and thus less available as co-factor for the enzyme activity of ribonucleotide reductase or complex IV of the respiratory chain, among other iron-containing proteins.
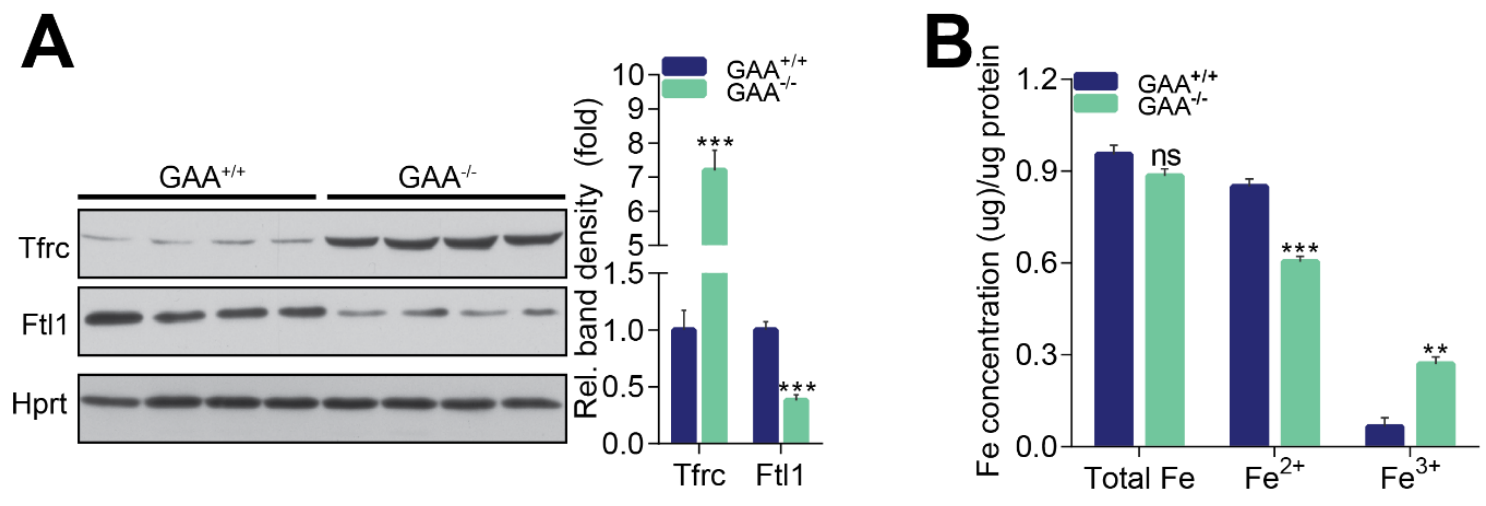

Figure 43| Impaired iron homeostasis in GAA loss - A Levels of transferrin receptor are increased while those of ferritin light chain are reduced in the cortex of $\mathrm{GAA}^{-}$- mice $(\mathrm{N}=8)$. B While total iron concentration is not altered by GAA loss, levels of ferrous iron $\left(\mathrm{Fe}^{2+}\right)$ are reduced and those of ferric iron $\left(\mathrm{Fe}^{3+}\right)$ are rather increased in $\mathrm{GAA}^{-} \% \mathrm{MEFs}(\mathrm{N}=5)$. Graphs represent mean \pm SEM for indicated number of samples; $p<0.01(* *), p<0.001\left({ }^{* * *}\right)$, and $p>0.05$ (ns); Student's tests; SEM: standard error of the mean, ns: not statistically significant.

\subsubsection{Iron deficiency causes mtDNA depletion}

Although it was shown that lysosomal malfunction due to GAA loss resulted in ferrous iron deficiency, it was essential to know whether iron deficiency impacted mtDNA copy numbers. To address this, control MEFs were treated with a prescribed iron chelator, deferoxamine following which levels of mtDNA copy number were measured. Accordingly, deferoxamine treatment resulted in increased TFRC expression and decreased levels of FTH1 and FPN (Figure 44A). FPN encodes for the cellular iron exporter ferroportin, whose levels, like those of ferritin, are reduced under conditions of iron deficiency. Consistently, mtDNA copy numbers were lowered in deferoxamine-treated cells to a degree similar to the $\mathrm{GAA}^{-1-} \mathrm{MEFs}$ (Figure 44B). To exclude drug-specific effects on mtDNA copy number levels, MEFs from IRP $1^{-1-}$ and IRP2 $2^{-1-}$ mice were tested for mtDNA abundance. IRP1 and IRP2 encode for iron-responsive elementbinding proteins, which regulate iron homeostasis. Lack of these proteins impairs iron homeostasis leading to reduced bioavailability of $\mathrm{Fe}^{2+}$ (Meyron-Holtz, 2004). 
Remarkably, mtDNA copy number levels were found to be significantly reduced in IRP $1 / 2^{-/-}$ MEFs (Figure 44C).
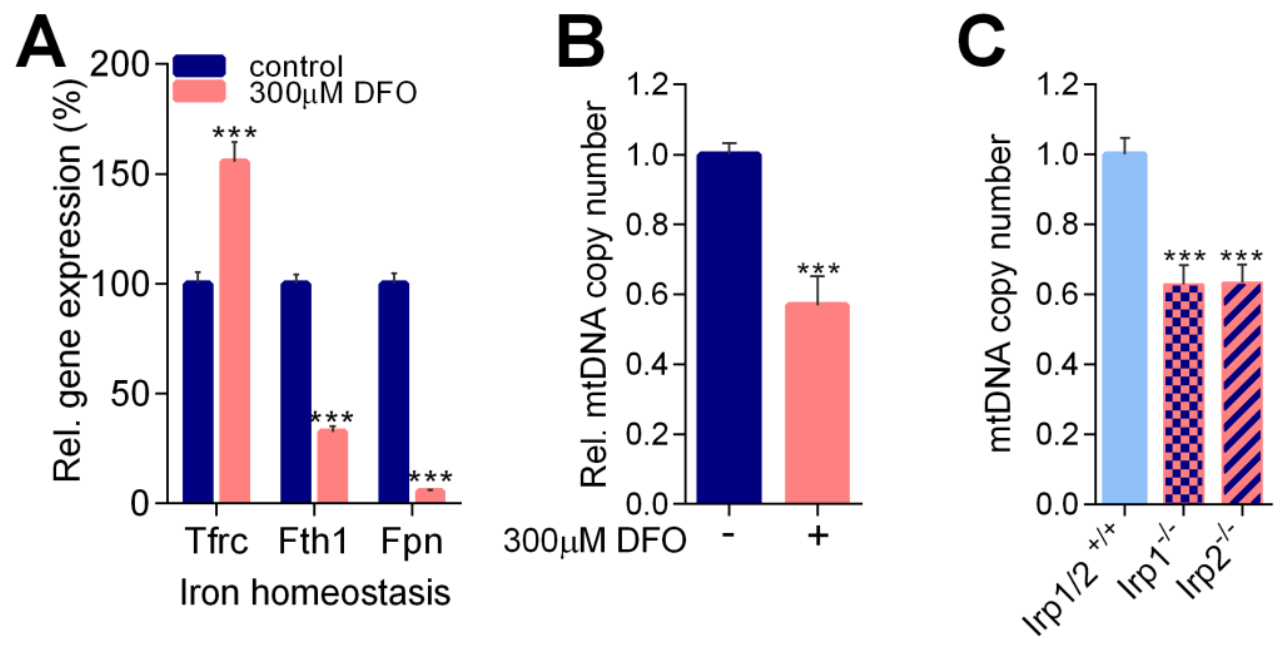

Figure 44| Iron deficiency results in mtDNA depletion - A TFRC transcripts are increased and FTH1 and FPN mRNAs are reduced following deferoxamine (DFO) treatment. B DFO treatment leads to mtDNA copy number loss in control MEFs. C Loss of IRPs, which triggers functional iron deficiency, results in mtDNA depletion Gene expression and mtDNA copy number are depicted as mean \pm SEM, $N=8 ; p<0.001\left(^{* * *}\right)$; Student's tests; SEM: standard error of the mean

More importantly, to show the dependency of mtDNA copy number defects on iron deficiency, we were curious to know whether iron supplementation would ameliorate mtDNA copy number loss in lysosomal malfunction. To this end, $\mathrm{GAA}^{-/-}$and wild-type MEFs were treated with either ferric iron diluted in sodium citrate, or with sodium citrate as a control. In line with our premise, iron repletion was enough to rescue mtDNA copy number defects in GAA ${ }^{-/-}$MEFs (Figure 45). These results, altogether, imply that lysosomal malfunction impairs iron homeostasis leading to reduced bioavailability of ferrous iron for processes necessary for mtDNA synthesis and mitochondrial function. 


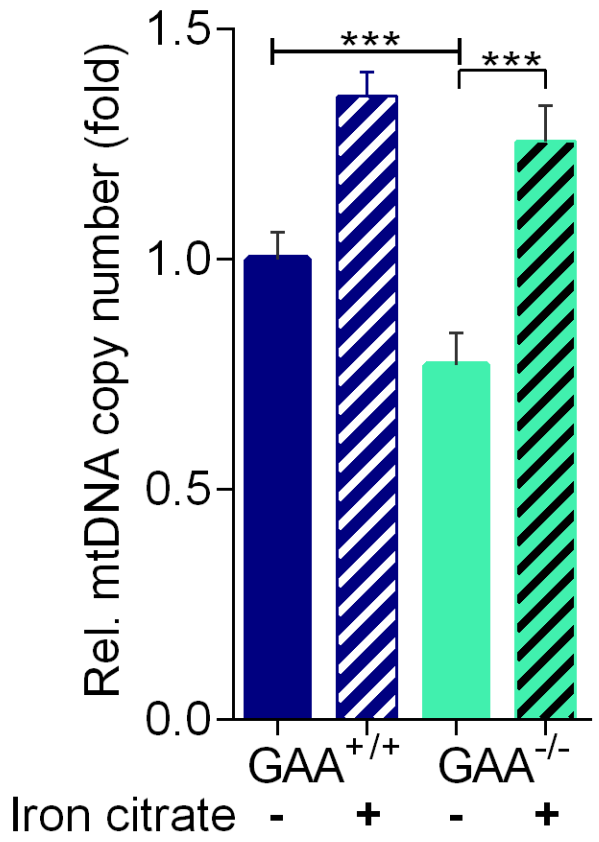

Figure 45| Iron supplementation rescues mtDNA copy number depletion - Repletion of iron via iron citrate supplementation rescues mtDNA defects in GAA $\%$ MEFs. $p<0.001{ }^{* * *}$ ); one-way ANOVA; mean \pm SEM, $\mathrm{N}=8$; SEM: standard error of the mean.

\subsection{7 mtDNA defects due to iron deficiency trigger the innate immune response}

Next, it was necessary to elucidate the biological relevance of these findings for the health (or disease) implications of an organism. As such, we looked for the role of mtDNA defects in disease pathogenesis. In addition to its vital role in encoding for key subunits of the respiratory chain, it is now appreciated that the mitochondrial genome induces the innate immune response through its cooperation with several pattern recognition receptors (Barbalat et al., 2011; Oka et al., 2012; West et al., 2015). It is thus not a coincidence that a hallmark of LSDs and neurodegenerative disorders is the induction of inflammation (reviewed in Bosch and Kielian, 2015; Vitner et al., 2015). The endolysosomal system, which degrades mitochondrial proteins and mtDNA during mitophagy, has evolved as a platform where pattern-recognition receptors like the toll-like receptor 9 (TLR9) sense DNA and subsequently activate the innate immune response (Kawai and Akira, 2010). 

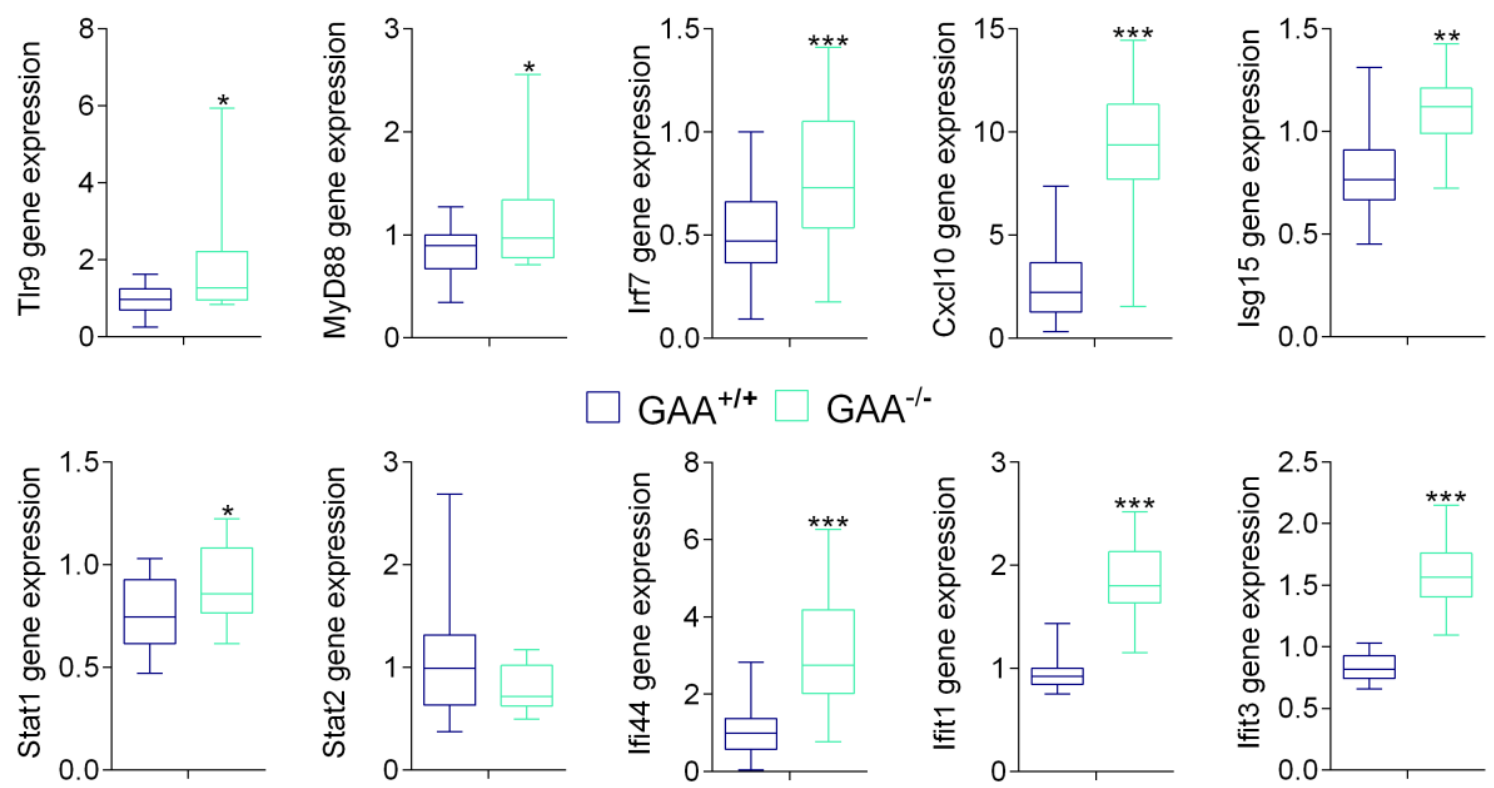

$\square \mathrm{GAA}^{+/+} \square \mathrm{GAA}^{-/-}$
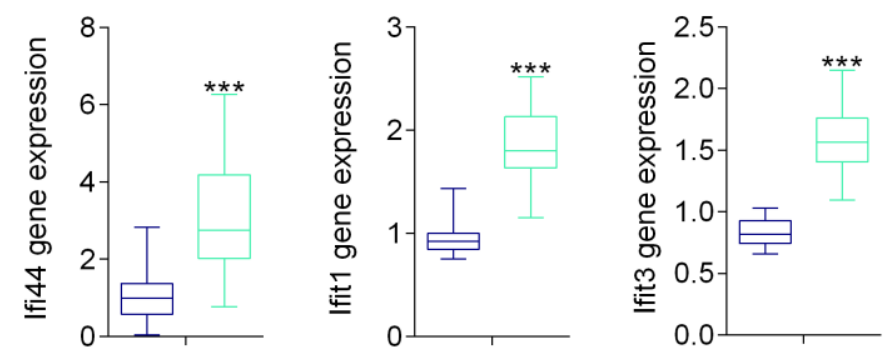

Figure 46| Induction of the innate immune response in the brain - Loss of GAA in the cortex triggers a transcriptional response of innate immune response activation. Gene expression is depicted as box plots showing minimum, mean and maximum expression levels, $\mathrm{N}=8 ; \mathrm{p}<0.05\left(^{*}\right), \mathrm{p}<0.01\left(^{* *}\right)$, and $\mathrm{p}<0.001\left(^{* * *}\right)$; Student's t-test

Consistent with these reports, we hypothesized that in lysosomal malfunction, the increased retention of agonists of TLR9 like mtDNA or its fragments in the lysosome would enhance the activation of the innate immune response pathway typically through increased gene expression of type I interferon genes. The expression of such genes was thus measured in the cortex and in the heart of $\mathrm{GAA}^{-/-}$mice. Strikingly, the expression of TLR9, its signaling pathway mediators, MyD88, IRF7, ISG15 and STAT1, and its downstream effector molecules, CXCL10, IFI44, IFIT1, and IFIT3 were all increased in the brain (Figure 46) and in the heart (Figure 47) of GAA' I- mice. 

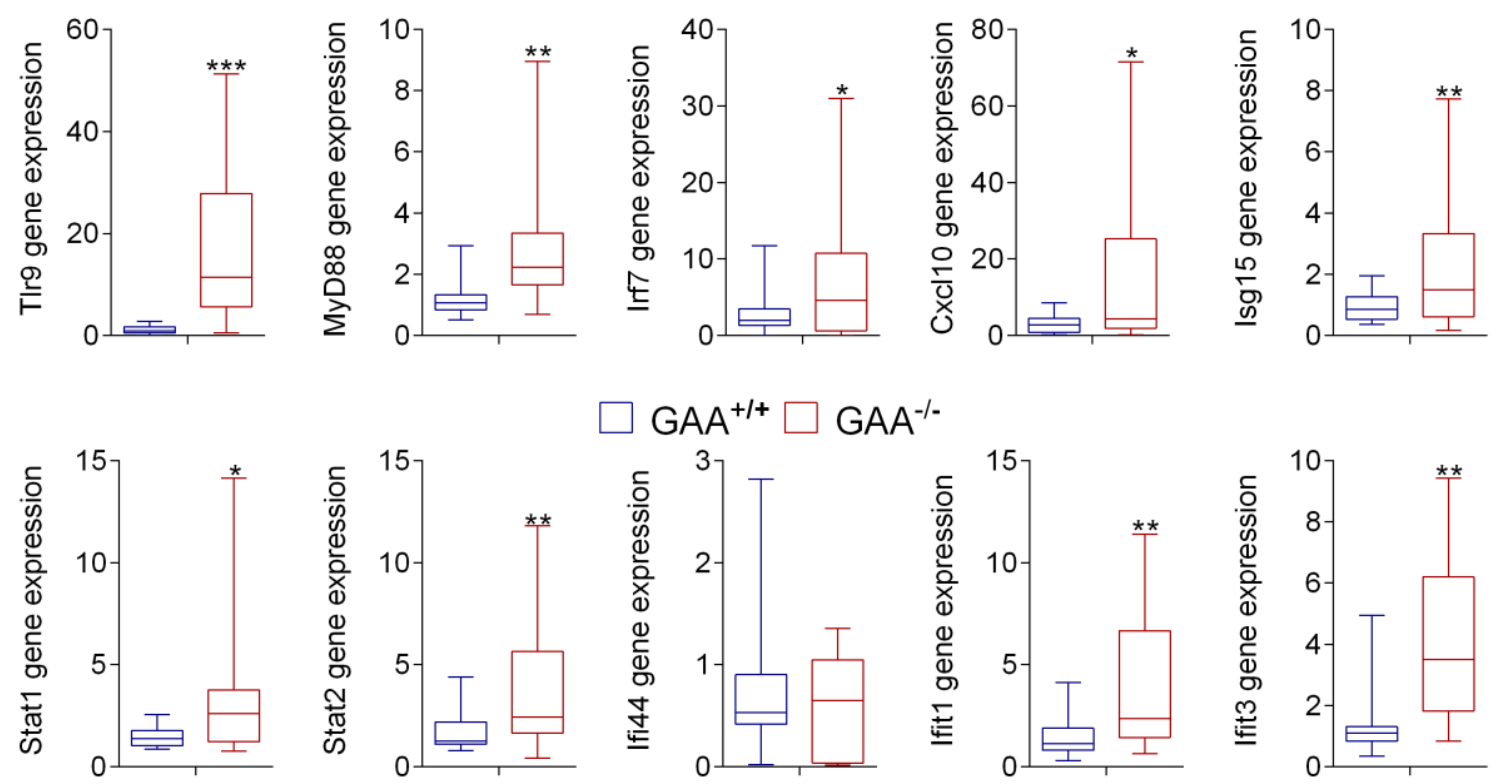

Figure 47| Cardiac innate immune response induction - GAA loss in the heart, like in the brain, induces the innate immune response via transcriptional activation of Type I interferon genes. Gene expression is depicted as box plots showing minimum, mean and maximum expression levels, $\mathrm{N}=8 ; \mathrm{p}<0.05\left(^{*}\right), \mathrm{p}<0.01\left(^{* *}\right)$, and $\mathrm{p}<0.001\left(^{* * *}\right)$; Student's t-test

\subsubsection{Increased gliosis in the cortex of $\mathrm{GAA}^{-/-}$mice}

The transcriptional induction of the innate immune response (Figures 46 and 47) prompted a further probing of markers of inflammation in $\mathrm{GAA}^{-/}$mice. In the context of neuroinflammation, the participation of glial cells is essential. The most abundant cell type in the brain, astrocytes, in addition to providing neurotrophic support to neurons, have been known for a long time to mediate immune responses in the CNS through chemokine secretion (Persidsky et al., 1999). Microglia, the chief immune cells of the CNS, can sense agonists via Toll-like receptors and other pattern-recognition receptors (Facci et al., 2014; Yao et al., 2013). When activated, microglia release chemokines and cytokines, which respectively recruit and activate peripheral immune cells. In support of this, preliminary results from magnetic resonance spectroscopy (MRS) of $\mathrm{GAA}^{-/}$mice at 6 months of age indicated a significant reduction in $\mathrm{T}_{2}$ relaxation times of water protons in the cortex, striatum and thalamus (Table 17), suggesting the infiltration of glial cells or macrophages in the brain of $\mathrm{GAA}^{-/-}$mice (Watanabe et al., 2015). 
Table 17: T2 relaxation times $(\mathrm{ms})$ of water protons

\begin{tabular}{lll}
\hline \hline Brain region & Wild-type & $\mathrm{GAA}^{-/-}$ \\
\hline Cerebral cortex & $40.3 \pm 0.5$ & $38.3 \pm 0.8^{\star \star \star}$ \\
Striatum & $39.7 \pm 1.0$ & $37.3 \pm 0.8^{\star \star}$ \\
Thalamus & $38.4 \pm 1.0$ & $35.9 \pm 1.1^{*}$ \\
\hline \hline
\end{tabular}

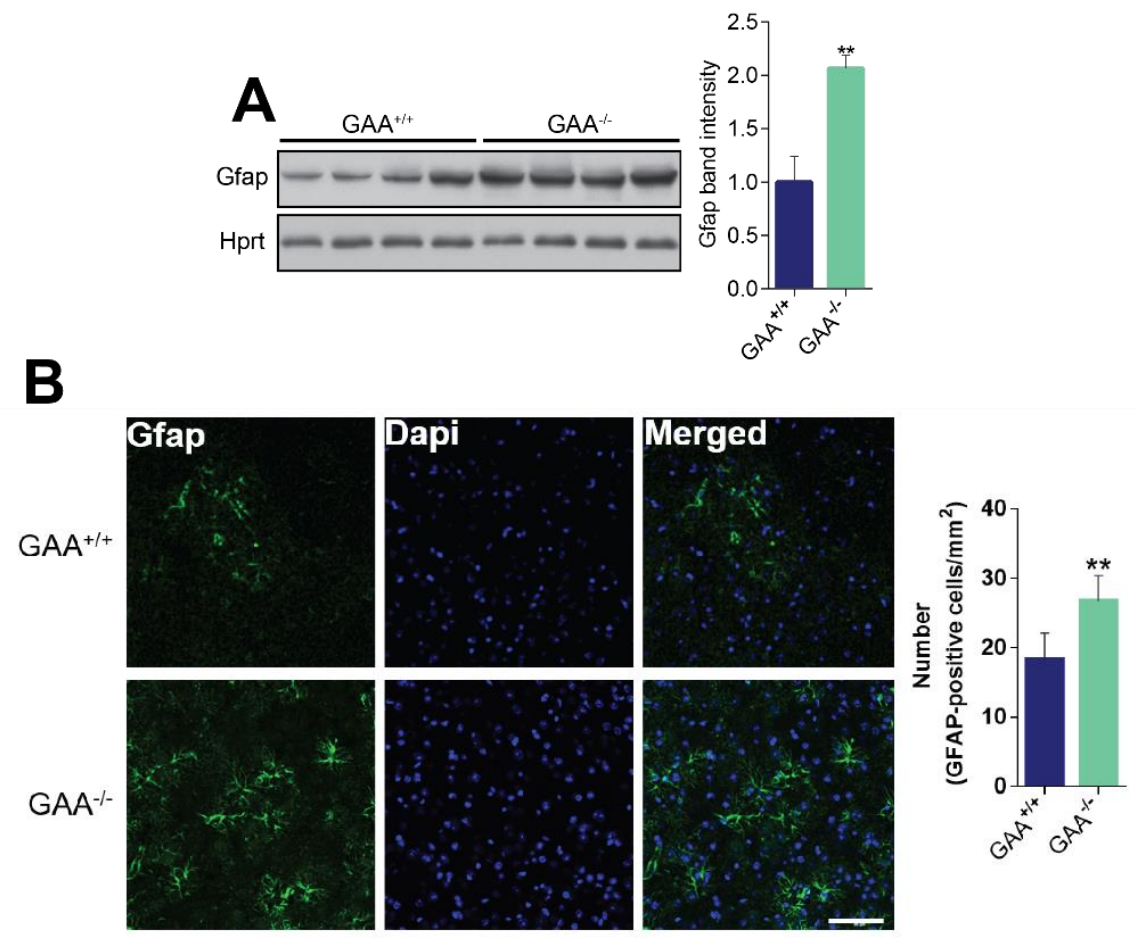

Figure 48| Glial cell proliferation in the cortex of GAA $/$ - mice - A Immunoblot analyses of whole cortical lysates show a two-fold increase in GFAP, a gliosis marker, levels in GAA $\%$ mice. B Number of GFAP-positive cells is increased in GAA $\%$ mice as demonstrated by increased GFAP immunostaining. Quantifications represent mean \pm SEM, N = 8; p <0.01 (**); Student's tests; SEM: standard error of the mean. Scale bars represent $20 \mu \mathrm{M}$.

This result would later be confirmed by immunoblots and immunostaining for the glial fibrillary acidic protein, GFAP, a gliosis marker, in the cortex of GAA ${ }^{-/-}$mice, where a two-fold increase in GFAP was observed (Figure 48A) with an increase in the number of GFAP-positive cells (Figure 48B). Thus far, this study showed that lysosomal malfunction triggers the innate immune response via a mechanism of mtDNA defects elicited by a pseudo-iron deficiency. 


\subsubsection{Iron supplementation ameliorates brain mtDNA defects and reduces} innate immune response in $\mathrm{GAA}^{-/-}$mice in vivo.

the mechanism elucidated in this study would be relevant for lysosomal storage and neurodegenerative disorders if it could be targeted to rescue some disease phenotypes, especially the inflammatory response. Although the goal of immune response induction is to swiftly contain an insult, typically as a protective response, persistent inflammation can exert harmful effects on the affected cells or tissues.
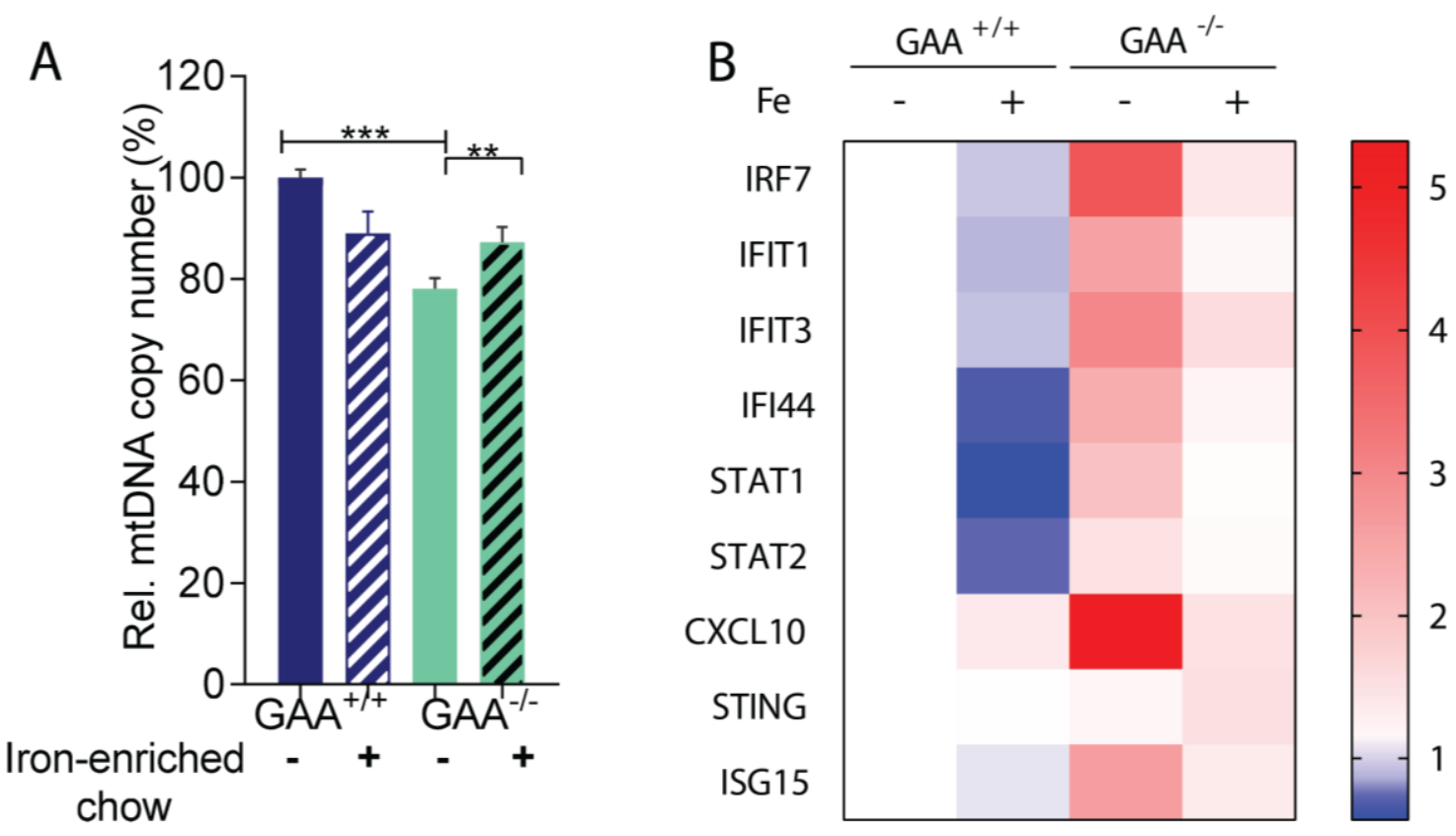

Figure 49| Iron supplementation rescues mtDNA defects and down-regulates innate immune activation - A In vivo iron supplementation via iron-enriched chow rescues mtDNA copy number decline in the cortex of GAA $\%$ mice at 2 months of age. $p<0.01(* *)$ and $p$ $<0.001\left(^{* * *}\right)$; one-way ANOVA; mean \pm SEM, $N=5$; SEM: standard error of the mean. B Iron supplementation dampens activated transcripts of innate immune response genes in the cortex of $\mathrm{GAA} \%$ mice. Heatmap represents average expression of each gene normalized to the expression levels of wildtype mice on standard chow, $\mathrm{N}=5$.

Given the progressive decline of mtDNA copy numbers in the brain of $\mathrm{GAA}^{-/}$mice (Figure 34), we postulated that persistent mtDNA-induced activation of the immune response would be detrimental to the affected tissues. It was also shown previously in cells that iron supplementation rescues mtDNA copy number defects (Figure 45). To this end, we were 
curious to decipher whether iron supplementation would indeed rescue mtDNA defects in vivo in $\mathrm{GAA}^{-/-}$mice and consequently ameliorate the induced immune response. Therefore, GAA ${ }^{-/-}$ mice and their corresponding wild-type littermates were fed with iron-enriched or standard chow ad libitum. The $\mathrm{GAA}^{-/-}$mice were mostly examined at 6 months of age. Nevertheless, we were curious about obtaining preliminary results on the iron supplementation experiments. As a result, a cohort of the mice was sacrificed at 2 months of age for mtDNA copy number measurements as well as gene expression profiling of the innate immune response pathway. Remarkably, iron supplementation was found to partially rescue mtDNA copy number levels in the cortex of $\mathrm{GAA}^{-/-}$mice already at 2 months (Figure 49A). Consequently, $\mathrm{GAA}^{-/-}$mice, with upregulated innate immune response genes, showed a decreasing trend in the expression of signaling and effector molecules of the innate immune response pathway following two months of iron supplementation (Figure 49B). This result warrants further analyses, which would be carried out following the 6 months of iron supplementation. 


\section{Discussion}

\subsection{Defective lysosomal signaling in Niemann-Pick disease represses mitochondrial biogenesis and function}

Mitochondrial dysfunction has been reported in patients and mouse models of the NiemannPick spectrum of lipid disorders, just like in most LSDs (Castillo et al., 2017; Osellame and Duchen, 2014). However, the mechanisms governing mitochondrial malfunction in these diseases are largely unknown. This study uncovers a novel mechanism that links chronic lysosomal malfunction in Niemann-Pick disease to mitochondrial dysfunction. We demonstrate unequivocally that the induction of transcriptional regulators KLF2 and ETV1, and their cooperation to repress mitochondrial biogenesis and mitochondrial function in Niemann-Pick disease.

\subsubsection{Mitochondrial biogenesis is repressed in Niemann-Pick disease}

We uncovered reduced mitochondrial respiration and increased levels of mitochondrial superoxides in fibroblasts from patients with Niemann-Pick Type A/B (Figure 13). This outcome was replicated in human dermal fibroblasts treated with an inhibitor of acid sphingomyelinase, the protein deficient in Niemann-Pick Type A/B. In addition, we showed that mitochondrial function is compromised in mouse embryonic fibroblasts prepared from NPC $1^{-}$mice, the model for Niemann-Pick Type C. Thus far, the mitochondrial impairments in lysosomal diseases have been attributed only to defective autophagy (mitophagy) and the consequent accumulation of dysfunctional mitochondria (Baulies et al., 2016; Ordonez, 2012).

However, in addition to defective autophagy, our results indicate that mitochondrial dysfunction in Niemann-Pick disease is a consequence of a signaling mechanism that represses mitochondrial biogenesis. Interestingly, this transcriptional response was found in cells and tissues of Niemann-Pick disease (Figures 14 and 19). Furthermore, repression of mitochondrial biogenesis in the brain and liver of NPC1/- mice was evident in only symptomatic mice (Figure $19 \mathrm{~B}, \mathrm{C})$ probably as an adaptive response to progressive lysosomal malfunction. It is noteworthy that lysosomal biogenesis was increased in all models of Niemann-Pick disease tested, a response that is usually seen as compensatory in LSDs (Karageorgos et al., 1997). Although the etiology is different for the subtypes of Niemann-Pick disease, our results underscore a significant overlap in the pathological mechanisms of the syndrome as reported 
previously (Vanier, 2013). Niemann-Pick Type A/B affects sphingolipid catabolism while Niemann-Pick Type $C$ is regarded as a sphingomyelin storage disorder, which mainly affects cholesterol efflux from the lysosome (Platt, 2014). However, the only approved therapy for Niemann-Pick Type C, miglustat, blocks glycosphingolipid biosynthesis (Patterson et al., 2012), suggesting the involvement of sphingolipids in Niemann-Pick Type $\mathrm{C}$. This further underlies the mechanistic overlaps in Niemann-Pick disease.

\subsubsection{KLF2 and ETV1 mediate repression of mitochondrial biogenesis in}

\section{Niemann-Pick disease}

Beyond reporting mitochondrial defects in Niemann-Pick disease, this study further demonstrated that a coordinated transcriptional program mediated by transcriptional regulators, KLF2 and ETV1, repressed mitochondrial biogenesis in Niemann-Pick disease. Indeed, KLF2 and ETV1, which were induced in Niemann-Pick disease (Figure 23), were predicted to target respiratory chain genes by MatInspector analyses of promoters of mitochondrial respiratory chain genes. In line with this, genes under ETV1 transcriptional regulation were found to include several mitochondrial genes from ETV1 ChIP-Seq data (Figure 24). Consistently, silencing either KLF2 or ETV1 reversed the repression of mitochondrial biogenesis in Niemann-Pick disease and ultimately rescued mitochondrial respiration. Strikingly, knockdown of KLF2 or ETV1 was sufficient to rescue the levels of NRF1, a known master regulator of mitochondrial biogenesis (Kelly and Scarpulla, 2004). This result implicates a potential transcriptional regulatory network involving KLF2, ETV1, and NRF1 in controlling mitochondrial biogenesis. In addition, the activated signaling pathway involving KLF2 and ETV1 is operational in fibroblasts from Niemann-Pick Type A/B patients and in tissues of the Niemann-Pick Type C mouse model. This further underlines the biochemical similarities between the different variants of Niemann-Pick disease, which all accumulate cholesterol and sphingomyelin in the lysosome (Patterson et al., 2012). Previous predictions of the role of KLF2 and ETV1 in regulating mitochondrial biogenesis or function are worthy of mention. Doddaballapur et al. (2015) showed that increased KLF2 activity in endothelial cells is associated with decreased mitochondrial content, which can be reversed by KLF2 silencing. Interestingly, ETV1, which is highly expressed in cancers, notably prostate cancer, has been shown to drive reprogramming of metabolic pathways typically with increased glycolysis and reduced mitochondria function (Baena et al., 2013). 
Furthermore, activities of KLF2 and ETV1 may be associated with other hallmarks of NiemannPick disease. For example, liver disease is a prevalent phenotype of Niemann-Pick disease (Patterson et al., 2012). Intriguingly, KLF2 has been shown recently to negatively regulate liver regeneration through activin A induction (Manavski et al., 2017). Indeed, overexpressing KLF2 in liver sinusoidal endothelial cells reduced hepatocyte proliferation. In addition, oligodendrocyte differentiation is known to be coordinated by differential transcriptional regulation, which includes robust ETV1 downregulation (Dugas et al., 2006). Accordingly, hypomyelination and neurodegeneration, which are prevalent in Niemann-Pick disease (German et al., 2002; Patterson and Walkley, 2017), maybe to some extent linked to ETV1 induction. The role of ETV1 and KLF2 in neurodegeneration warrants further investigation.

\subsubsection{Mechanisms of KLF2 and ETV1 induction in Niemann-Pick disease}

Strikingly, silencing KLF2 results in ETV1 depletion and improved mitochondrial biogenesis and function, suggesting that KLF2 was potentially upstream of ETV1 (Figure $27 \mathrm{~A}$ ). In addition, ERK activity, a positive regulator of ETV1 (Janknecht, 1996; Janknecht, 2003), was increased in Niemann-Pick patient fibroblasts. Indeed, phosphorylation of ETV1 at residues serine-191 and serine-126 by ERK-dependent signaling drives ETV1 nuclear translocation and transcriptional activity (Janknecht, 2003). In line with this, ERK inhibition in Niemann-Pick patient cells, like KLF2 silencing, ablated ETV1 protein levels without altering KLF2 amounts (Figure 28). Taking also into account the report that KLF2 hemizygous mice present reduced ERK activity (Das et al., 2012), these data advocate that KLF2 cooperates with ERK to induce ETV1 and drive its transcriptional activation.

The mechanism mediating KLF2 induction in Niemann-Pick disease seems rather complex but we show that it is dependent on reduced sphingosine-1-phosphate (S1P) signaling. KLF2 is a known regulator of S1P signaling through the transcriptional activation of S1P receptor 1 (S1PR1) expression. Interestingly, S1P negative feedback signaling targets KLF2 for proteasomal degradation via AKT (Bai et al., 2007; Sinclair et al., 2008; Skon et al., 2013). Consistent with reduced S1P signaling due to decreased S1P levels in Niemann-Pick disease (Fan et al., 2013), we found reduced AKT activity, induced KLF2 levels and increased S1PR1 expression in Niemann-Pick patient fibroblasts. Conversely, enhanced agonist-mediated S1P signaling facilitated KLF2 downregulation and improved mitochondrial biogenesis and function (Figures 29 and 30). Remarkably, increased S1P signaling was recently found to maintain mitochondrial content of naïve $T$ cells and promote mitochondrial function (Mendoza et al., 2017). Although the mechanism was not demonstrated in this report, we propose that it is likely 
a consequence of KLF2 downregulation following S1P signaling. It is also noteworthy that impaired S1P signaling is associated with neurodegeneration, a prevalent phenotype in Niemann-Pick disease (German et al., 2002; Hagen et al., 2011).

Mitochondrial defects have been reported in the most common LSD, Gaucher's disease (Osellame et al., 2013). Mutations in the GBA1 gene, which result in defective lysosomal glucocerebrosidase in Gaucher's disease, have been identified as genetic rick factors for idiopathic Parkinson's disease (Bultron et al., 2010). It would be particularly interesting to understand whether KLF2 and ETV1 mediate mitochondrial defects in Gaucher's disease, given that lysosomal glucocerebrosidase activity is crucial for sphingosine formation.

Although we have shown that induction of KLF2 and ETV1 in Niemann-Pick disease represses mitochondrial biogenesis (Figure 50), it appears that this is an adaptive response to promote cell survival. KLF2 and ETV1 are anti-apoptotic, which is illustrated by their crucial role in cancers (Alberts-Grill et al., 2016; Chi et al., 2010; Ohguchi et al., 2016). Consequently, KLF2 and ETV1 induction in response to chronic lysosomal malfunction in Niemann-Pick diseases promotes cell survival at the expense of a loss of mitochondrial function. It is imaginable that by shutting down mitochondrial respiration and the TCA cycle, for instance, citrate can be shuttled into the cytoplasm for fatty acid synthesis. This would be relevant for Niemann-Pick disease, given that lipids accumulate in the lysosome and are probably not available to other cellular compartments.

Taken together, this work contributes to our understanding of the interplay between organelles by highlighting that mechanisms of communication between lysosomes and mitochondria go far beyond metabolic cues and involve complex signaling and transcriptional regulatory networks. We uncover a signaling pathway that engages transcriptional regulators, which mediate the repression of mitochondrial biogenesis and mitochondrial function in NiemannPick disease. The model for this pathway, as illustrated in Figure 50, shows that S1P, which is released from cells, stimulates a family of $G$ protein-coupled receptors, including S1PR1. S1PR1 mediates downstream signaling to induce biological processes such as growth and differentiation. Importantly, S1PR1 negative feedback signaling targets KLF2 for degradation. Owing to the reduced circulating S1P levels in Niemann-Pick disease, our model predicts the induction of KFL2 via reduced S1PR1 signaling. KLF2 in turn induces and cooperates with ETV1 in an ERK-dependent manner to target and downregulate the expression of mitochondrial genes. 
Interestingly, the model presented in Figure 50, also describes a novel therapeutic target that is potentially relevant for Niemann-Pick disease, which involves modulation of S1PR1 signaling. There is already considerable translational research on the potential of S1PR1 modulators in human diseases, especially in multiple sclerosis. Although the contribution of this work to such clinical investigations is barely relevant, our findings underlie an additional mechanism of S1PR1 signaling in regulating cellular functions, which may be useful in understanding some clinical outcomes of S1PR1 modulation.

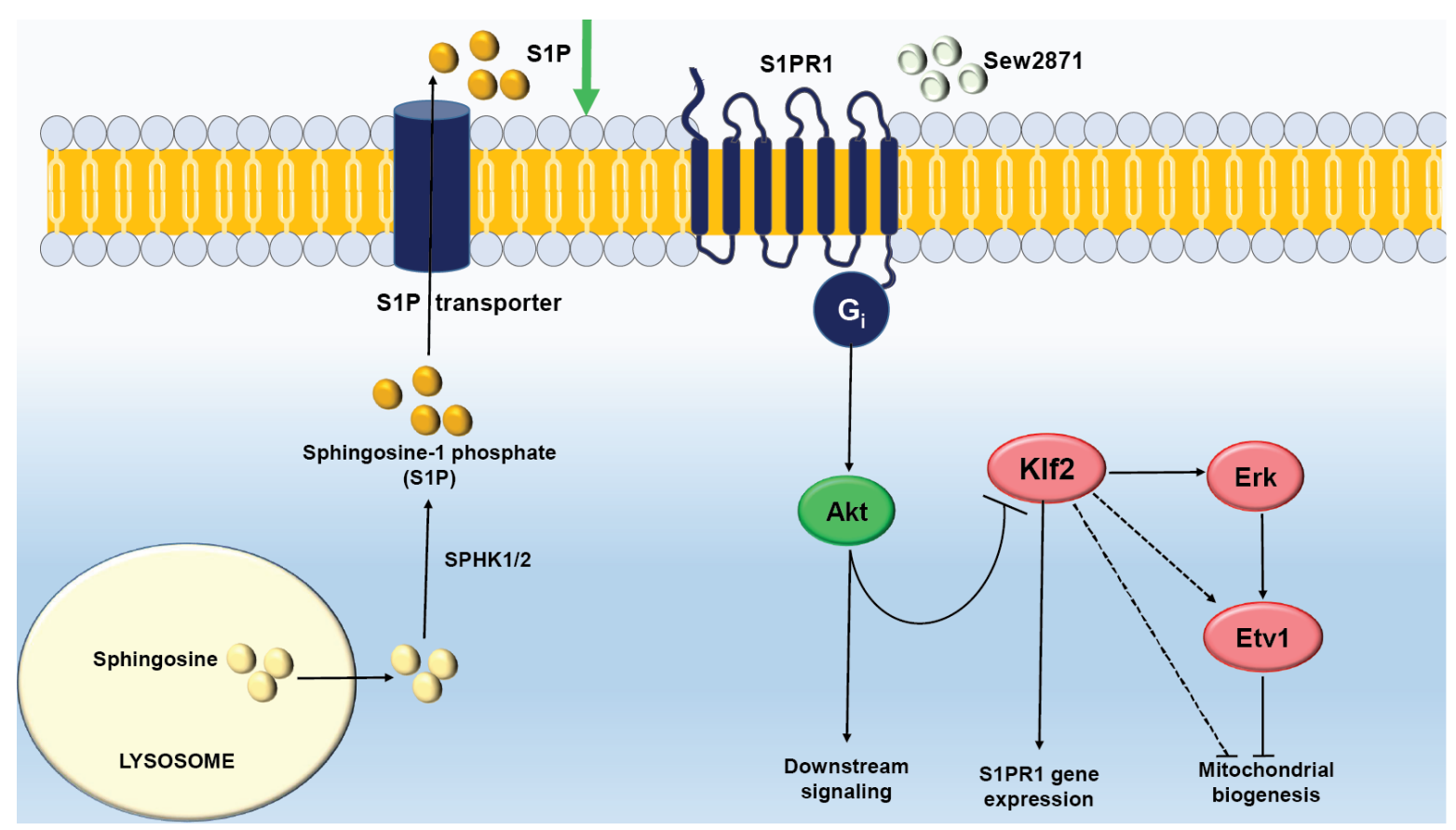

Figure 50| Model of lysosomal and mitochondrial crosstalk in Niemann-Pick

disease - Niemann-Pick disease is in part caused by decreased circulating S1P levels, culminating in its reduced signaling and the induction of ETV1 via ERK and KLF2. Induced KLF2 and ETV1 engage a transcriptional network that represses mitochondrial biogenesis and function in Niemann-Pick disease. (Original illustration, KF Yambire) 


\subsection{Perturbed iron homeostasis links lysosomal malfunction to mtDNA depletion and induction of the innate immune response}

Sphingolipid metabolism was found to be crucial for the mechanism of crosstalk between lysosomes and mitochondria in Niemann-pick disease. Impaired lipid metabolism is not implicated in all LSDs, however, suggesting that different mechanisms might mediate interplay between organelles in different LSDs. Nevertheless, overlaps in phenotypes between LSDs might also suggest common mechanisms which can be attributed to intra-lysosomal steric hindrances following substrate accumulation and consequent secondary enzyme deficiencies (Platt et al., 2012). This is further demonstrated by our findings in the mouse model of Pompe's disease (GAA ${ }^{-/}$mouse). Although Pompe disease is a glycogen storage disorder, we find that the Pompe's disease model shares common features with other LSDs while presenting distinct mechanisms and phenotypes of organelle communication.

\subsubsection{Mitochondrial genome defects in Pompe's disease}

It is worthy to underline that this study presents the first line of evidence of cerebral involvement in the pathomechanisms of Pompe's disease. The disease typically causes extensive glycogen accumulation with widespread neuromuscular impairments and cardiomyopathy. The only other evidence of CNS involvement was demonstrated recently in the spinal cord of GAA ${ }^{-/-}$mice by Fuller and colleagues (Turner et al., 2016). In addition to mitochondrial defects which were previously reported in striated muscles of $\mathrm{GAA}^{-/-}$mice (Lim et al., 2015), we report widespread mtDNA defects for the first time in brain and heart tissues of $\mathrm{GAA}^{-/-}$mice and in MEFs extracted from these mice. Strikingly, the decline in mtDNA copy numbers in the cortex and in the heart of $\mathrm{GAA}^{-/}$mice do not seem to be a consequence of mitochondrial biogenesis, which was decreased in the cortex but robustly induced in the heart of $\mathrm{GAA}^{-/-}$mice. The production of functional mitochondria requires cooperation between nuclear and mitochondrial genomes. As a result, an imbalance between the expression of nuclear- and mtDNA-encoded mitochondrial genes has detrimental consequences for cell survival. Larsson et al. (1998) showed that oxidative phosphorylation (OXPHOS) is markedly disrupted when mtDNA-encoded respiratory chain subunits are not expressed. Interestingly, selective transactivation of nuclear-encoded mitochondrial genes via c-myc results in the production of misassembed and defectvie OXPHOS complexes and leads to apoptosis induction (Morrish et al., 2003). Evidence of such 
imbalance may pertain to cardiac tissues (one of the most affected tissues) in Pompe's disease, which show reduced mtDNA copy number and increased mitochondrial biogenesis. It would be particularly curious to know whether imbalance between the expression of nuclear and mitochondrial genomes contributes to Pompe's disease pathology in the heart.

It is noteworthy that mtDNA depletion progressively worsened in the cortex as mice aged (Figure 34). Taken into consideration the reports of two independent studies that mtDNA copy number is reduced in patients with neurodegenerative disorders (Gui et al., 2015; Pyle et al., 2015), render our findings more remarkable. In addition, the role of lysosomal and mitochondrial crosstalk in neurodegenerative disorders (e.g. PD) has been known for some time now (Burbulla et al., 2017; Osellame et al., 2013). It is therefore likely that mtDNA defects sit at the crossroads between mitochondrial and lysosomal interplay in LSDs and neurodegenerative diseases.

\subsubsection{Mechanisms of mtDNA depletion in Pompe's disease}

Similarly to mtDNA depletion, TFAM protein amounts were decreased in all examined tissues and in MEFs of $\mathrm{GAA}^{-/-}$mice. Although this result initially presented a 'chicken-and-egg' problem, given that TFAM and mtDNA regulate the levels of each other, we demonstrated that mtDNA copy number decline was not a consequence of TFAM depletion. Indeed, restoring TFAM amounts to control levels by increasing mTORC1 activity had no noticeable impact on mtDNA copy number levels. As demonstrated by Matsushima et al. (2010), when not bound to mtDNA, TFAM is targeted for degradation by Lon proteases. This might account for the loss of TFAM in $\mathrm{GAA}^{-/-}$mice, especially since restored TFAM levels coincided with reduced Lon protease levels (Appendix Figure 57).

mtDNA depletion syndromes are not restricted only to mutations in genes that regulate mtDNA maintenance. Indeed, mutations in genes that regulate components of nucleotide metabolism have been implicated in mtDNA depletion syndromes (reviewed in El-Hattab et al., 2017), suggesting that nucleotide availability is crucial for mtDNA maintenance and stability, especially in post-mitotic cells like neurons. This study demonstrated that mtDNA defects were prevalent only under conditions where cell division was slowed down or halted. Subsequent metabolite profiling of cells with downregulated GAA levels showed impaired nucleotide metabolism with reduced concentrations of intermediates of purine and pyrimidine metabolic pathways. Metabolic profiling of Pompe's disease cells was recently published (Sato et al., 2016), and findings like increased glycolysis and increased nitrogen metabolism were corroborated by our study. Contrary to our findings, purine metabolism was found in this report to be increased in 
Pompe's disease cardiomyocytes. Given that the cardiomyocytes used in Sato et al. (2016) were derived from induced pluripotent stem cells prior to metabolomic profiling, it is likely that metabolic reprogramming during differentiation influenced some of the outcomes in this study. Nevertheless, we enhanced nucleotide metabolism via folic acid supplementation. The folate cycle shuttles one-carbon units to specific biosynthetic enzymes, which are involved, for example, in purine and in deoxythymidine monophosphate synthesis (Nilsson et al., 2014). Folate supplementation induced the levels of MTHFD2, whose bi-functional activity in folate metabolism is crucial also for nucleotide biosynthesis (Di Pietro et al., 2004). Remarkably, mtDNA defects in $\mathrm{GAA}^{-/-}$MEFs were rescued following folate supplementation. Interestingly, in the "Deletor" mouse, a model for adult-onset mitochondrial myopathy, Nikkanen et al. (2016) showed that mtDNA maintenance defects resulted in increased demand for one-carbon units for purine and methionine synthesis. The increased demand for nucleotides ultimately remodeled cytoplasmic metabolic pathways and contributed to myopathy. Intriguingly, myopathy is a hallmark of Pompe's disease. Therefore, it is plausible that the beneficial effects of folate supplementation in mtDNA defects stem from a negative feedback loop, where folate rapidly makes nucleotide available, lowers the excessive demand for one-carbon metabolism and dampen metabolic remodeling. It is noteworthy that folate supplementation had previously been shown to ameliorate mitochondrial and mtDNA defects in a Drosophila model of Parkinson's disease (Tufi et al., 2014). It would be particularly relevant to study the role of deoxyribonucleotide (dNTP) supplementation in reversing mtDNA depletion in Pompe's disease. This is especially interesting, since dNTP supplementation bypasses the need for folate metabolism and nucleotide biosynthesis.

\subsubsection{Lysosomal malfunction impairs iron homeostasis}

Although the activity of ribonucleotide reductases is crucial for nucleotide metabolism, especially for mtDNA replication in quiescent cells (Pontarin et al., 2012), no differences in the levels of ribonucleotide reductase subunit p53R2 were found in $\mathrm{GAA}^{-/-}$mice and their wild-type littermates. Nevertheless, the activity of ribonucleotide reductases depends largely on iron bioavailability, a required co-factor for initiation of nucleotide reduction (Bourdon et al., 2007; Guittet et al., 2001). In line with this, results from two independent experiments suggested iron deficiency in $\mathrm{GAA}^{-/}$mice. Despite retaining increased levels of the catalytic subunit of respiratory chain complex IV, cortical mitochondria from $\mathrm{GAA}^{-/-}$mice showed about $50 \%$ reduction in complex IV activity staining. The most likely basis for this observation is reduced 
bioavailability of iron, which is an essential component of heme, a required protein for electron transfer to molecular $\mathrm{O}_{2}$. Furthermore, the typical cellular response to iron deficiency, increased transferrin receptor levels and decreased ferritin levels were observed in the cortex and in MEFs of $\mathrm{GAA}^{-/}$mice. In addition, functional iron deficiency was confirmed in $\mathrm{GAA}^{-/} \mathrm{MEFs}$ by the findings that cellular ferrous iron $\left(\mathrm{Fe}^{2+}\right)$ concentrations were decreased while total iron concentrations were unchanged in $\mathrm{GAA}^{-/-}$cells. Altogether, these results implicate lysosomal dysfunction in impaired iron homeostasis since the reduction of $\mathrm{Fe}^{3+}$ to $\mathrm{Fe}^{2+}$ occurs in the lysosome, and lysosomal activity mediates the release of free iron to the cytoplasm (Rouault, 2013).

Iron, which forms part of heme and iron-sulfur clusters, is an indispensable trace element for mammalian metabolism, and functions as a cofactor in several metabolic reactions. Interestingly, iron deficiency and iron overload have detrimental consequences for cellular metabolism and survival. Iron deficiency was recently demonstrated to cause dopaminergic neurodegeneration in mice (Matak et al., 2016), while the role iron overload, in oxidative stress via the Fenton reaction has been well described (Winterbourn, 1995). As a result, understanding iron metabolism and the mechanisms of iron homeostasis, especially in LSDs, is becoming increasingly relevant, since mounting evidence suggests impaired iron handling in the pathogenesis of some LSDs. For instance, clinical manifestations of most LSDs in the newborn include anemias, which are dependent on iron availability (Staretz-Chacham et al., 2009). In addition, impaired myelination was recently associated with functional iron deficiency in a mouse model of mucolipidosis type IV (Grishchuk et al., 2015). The lysosomal lumen serves as a major catabolic hub for several macromolecules, including iron-containing proteins, such as ferritin and OXPHOS complexes. Owing to this role, impaired lysosomal iron handling in LSDs is now recognized as a 'double-edged sword' (Kurz et al., 2008). On one hand, accumulation of iron in the lysosome compounds lysosomal deficits in LSDs and has been shown to initiate apoptosis or necrosis through lysosomal membrane permeabilization (Kurz et al., 2008; Zhao et al., 2003). On the other hand, lysosomal build-up of iron makes it less available to be incorporated into the several iron-containing proteins and as a cofactor in metabolic reactions (Kurz et al., 2008). It is therefore plausible that lysosomal mishandling of iron in $\mathrm{GAA}^{--}$mice contributes to the reduced activity of complex IV of the respiratory chain and mitochondrial dysfunction, as well as reduced ribonucleotide reductase activity and impaired nucleotide metabolism. Although our findings highlight a role of iron in linking lysosomal malfunction to mitochondrial dysfunction in Pompe's disease, the mechanism involved warrants further investigation. 


\subsubsection{Cellular iron levels impact mtDNA copy numbers}

In addition, the implications of the role of iron in ribonucleotide reductase activity suggest that modulating iron levels in cells should be sufficient to impact mtDNA copy numbers. In line with this reasoning, deferoxamine-mediated iron deficiency resulted in a robust decline in mtDNA copy numbers in control cells. Interestingly, deferoxamine, which is a prescribed iron chelator for iron overload in humans, has been shown to mainly act by chelating lysosomal iron (Doulias et al., 2003). This further illustrates the role of lysosomal iron in mtDNA defects in $\mathrm{GAA}^{-/-}$mice. Likewise, MEFs extracted from IRP1 ${ }^{-/-}$and IRP2 $2^{-/-}$mice that specifically present functional iron deficiency had decreased mtDNA copies, thereby confirming the role of iron in mtDNA stability. Conversely, iron repletion, like folate supplementation, rescues mtDNA defects in GAA ${ }^{-/-}$MEFs. mtDNA maintenance is a tightly regulated process and defects in mtDNA have been linked to several human mitochondrial diseases (DiMauro and Davidzon, 2005). It is therefore striking how iron levels adequately play such a crucial role in regulating mtDNA copy number level, given that modulation of iron levels is common in clinical practice. Our findings in Pompe's disease implicate iron and folate metabolism in mtDNA defects. It would be particularly interesting to understand the mechanistic connection between iron and folate metabolism.

\subsubsection{Induction of innate immune response in $\mathrm{GAA}^{-/-}$mice}

mtDNA was first demonstrated by Zhang et al. (2010) to contain damage-associated molecular patterns (DAMPs) which are sensed by TLR9 with a subsequent induction of the innate immune response. Accordingly, it is expected that mtDNA defects would trigger the innate immunity. Consistently, this study demonstrates by three independent experiments that innate immune response induction is prevalent in $\mathrm{GAA}^{-/-}$mice. We found increased gene expression of all components of the TLR9-mediated pathway of innate immune response activation in the brain and in the heart of $\mathrm{GAA}^{-/-}$mice. Interestingly, this response was found to be likely dependent on TLR9 (and its agonist, mtDNA), because $\mathrm{GAA}^{-/-}$MEFs, which poorly express functional TLR9 (Ewald et al., 2008), did not show induction of the immune response, despite displaying mtDNA defects. Increased expression of cytokines and chemokines following TRL9 signaling serve to recruit immune cells to the site of DAMPs. This was supported by evidence of immune cell infiltration in the CNS by magnetic resonance spectroscopy. Indeed $\mathrm{T}_{2}$ relaxation time of water protons was decreased in the cortex, striatum, and thalamus of $\mathrm{GAA}^{-/-}$mice (Table 16). Reduced $\mathrm{T}_{2}$ relaxation time of water protons has previously been shown to be a reliable in vivo index of macrophage or glial cell infiltration in the brain (Watanabe et al., 2015). 
In addition, gliosis was confirmed in $\mathrm{GAA}^{-/-}$mice, which showed an increase in the number of GFAP positive cells in the cortex. Given that distinct cell populations are implicated in the induction of gliosis, including microglia and astrocytes, it is of utmost priority to identify which glial cell type is activated in the cortex of $\mathrm{GAA}^{-/-}$mice. After demonstrating mtDNA defects in a lysosomal storage disorder, and taking into account other reports, which show mtDNA defects in neurodegenerative diseases (Gui et al., 2015; Keogh and Chinnery, 2015; Pyle et al., 2015), it is not surprising that induction of the inflammatory response is a hallmark of most LSDs and neurodegenerative diseases (reviewed in Bosch and Kielian, 2015; Vitner et al., 2015).

\subsubsection{Iron rescues mtDNA defects and induced immune responses}

We demonstrate that the mechanism of mtDNA copy number decline and its consequences in $\mathrm{GAA}^{-/-}$mice could be targeted to ameliorate the observed defects. We showed by preliminary evidence in $\mathrm{GAA}^{-/-}$mice at 2 months of age that in vivo dietary iron supplementation rescues mtDNA defects in the cortex. Interestingly, reversing mtDNA defects in $\mathrm{GAA}^{-{ }^{-}}$mice also decreased the previously induced innate immune response, further validating the mechanism of mtDNA defects that we uncover in this study. In addition, the persistence of lysosomal malfunction implies that if not modulated, mtDNA defects would continually trigger the immune response resulting potentially in chronic inflammation, which is known to be deleterious for affected tissues and cells (Oka et al., 2012). Although remarkable, it must be emphasized that these results are preliminary since all in vivo experiments in this study were carried out with mice at 6 months of age. Results at 6 months following dietary iron supplementation in GAA ${ }^{-/}$ mice will be communicated in due course.

Taken together, the results of this study establish that mtDNA intersects both mitochondria and lysosomes and that iron plays a crucial in linking lysosomal dysfunction to mtDNA defects as illustrated in Figure 51. This model posits that impaired iron homeostasis, due to lysosomal malfunction in Pompe's disease, results in reduced bioavailability of iron, which affects the function of iron-containing proteins, including respiratory chain complexes and ribonucleotide reductases. The ensuing mitochondrial stress due to respiratory chain malfunction, and reduced activity of ribonucleotide reductases, ultimately affect mtDNA maintenance leading to its loss. Circulating mtDNA reaches the lysosome via the endocytic pathway, while mtDNA contained in mitochondria reaches the lysosome via mitophagy. Within the lysosome, mtDNA acts as agonist of the TLR9 signaling pathway to induce innate immunity. Based on the findings of this study and other reports, we propose that modulating mtDNA defects using iron levels 
may provide crucial therapeutic avenues for LSDs and probably neurodegenerative diseases. The results of this study show the potential of modulating iron levels to enhance nucleotide metabolism and reverse mtDNA defects, and improve mitochondrial respiratory chain function. This study also uncovers the mechanism by which iron plays a crucial role in signaling between mitochondria and lysosomes through the tightly regulated mtDNA molecule.

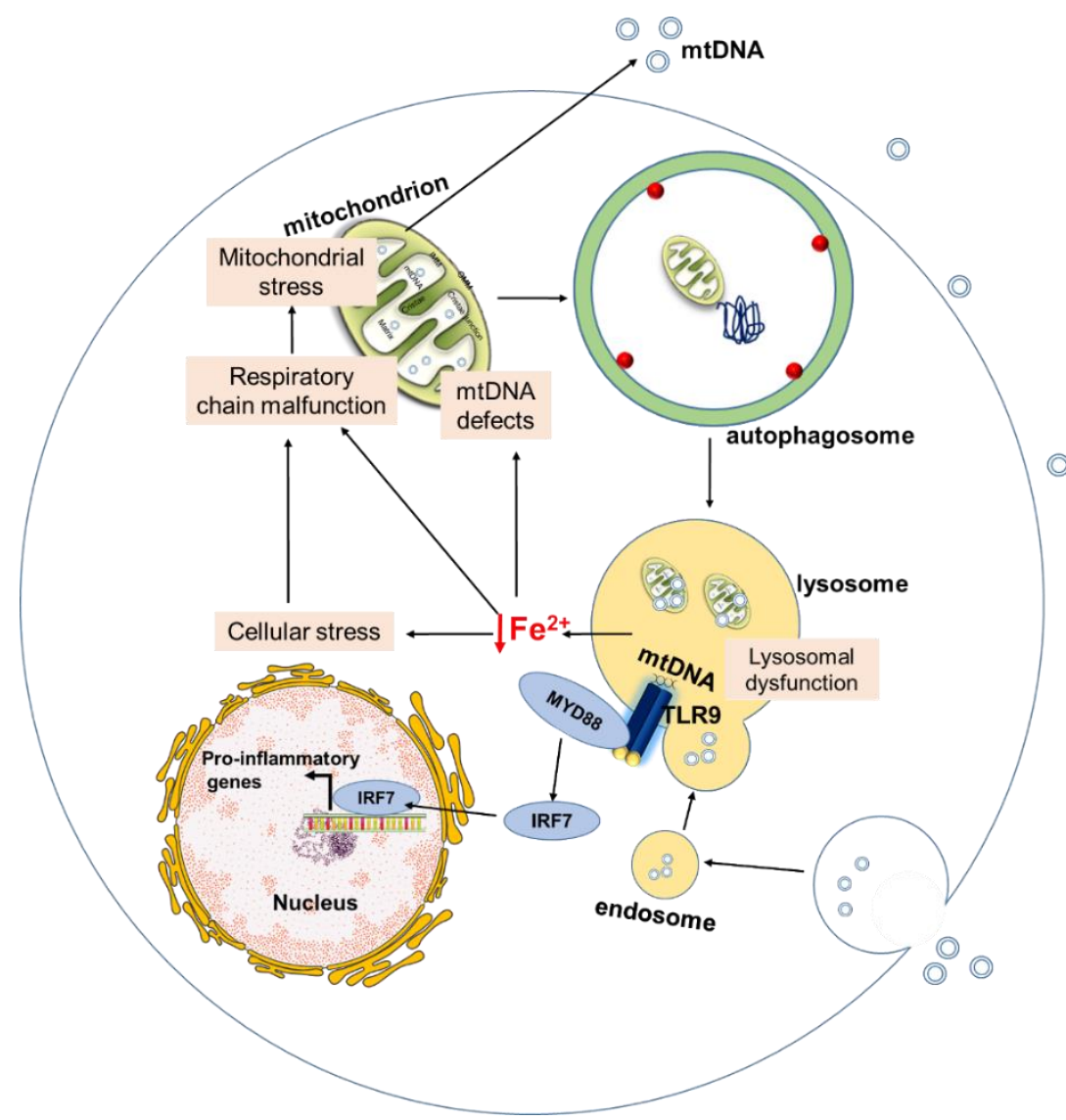

Figure 51| Mechanism of lysosomal-mitochondrial crosstalk in Pompe's disease Chronic lysosomal malfunction impairs iron homeostasis resulting in functional iron deficiency, which impairs mitochondrial function and nucleotide bioavailability for mtDNA synthesis in quiescent cells. This results in mtDNA instability and depletion. mtDNA consequently reaches the lysosome, via the endocytic and/or autophagy pathways, where it acts as DAMPs to trigger TLR9-MYD88-IRF7 pro-inflammatory signaling.( Original illustration, KF Yambire) 


\section{Summary and conclusion}

Distribution of cellular functions in membrane-bound organelles such as the nucleus, mitochondrion, and lysosome is a hallmark of eukaryotic life. Contrary to earlier views that organelles were functionally isolated from each other, unequivocal evidence nowadays suggests the existence of interdependent networks between these membrane-limited cellular compartments. It is therefore likely that the interplay between these organelles plays a crucial role in maintaining organelle homeostasis and contributes to pathologies that arise due to organelle dysfunction. As a result, understanding the crosstalk between organelles has become of utmost priority to cell biology.

The findings of this thesis deepen our understanding of the mechanisms of communication between lysosomes and mitochondria, and illustrates further that disease pathogenesis that arise due to organelle dysfunction engages the crosstalk between organelles as well. Based on two different and etiologically distinct LSDs (lipid storage disorder and glycogen storage disorder), this study established that lysosomal dysfunction affects mitochondrial homeostasis and function at several levels including transcriptional, mtDNA maintenance and ultimately functional levels. Interestingly, we had shown previously that mitochondrial malfunction affects transcriptional programs of lysosomal biogenesis (Fernández-Mosquera et al., 2017), further illustrating that organelle crosstalk via transcriptional regulation may be a widespread mechanism. The study further elucidates the underlying mechanisms that link the different LSD models to mitochondrial deficits.

In the context of Niemann-Pick diseases, the findings of this study established how impaired sphingosine-1-phosphate signaling, which arises primarily due to impaired catabolic activity of lysosomal sphingomyelinases, engages transcriptional repressors of mitochondrial biogenesis, KLF2, and ETV1. In addition, the mechanisms of KLF2 and ETV1 induction were demonstrated, and these mechanisms highlighted the therapeutic potential of sphingosine-1-phosphate modulation in ameliorating features of Niemann-Pick diseases. Interestingly, modulators of sphingosine-1-phosphate signaling have been the subject of phase III clinical trials in multiple sclerosis, which exacerbates some features of Niemann-Pick disease. In closing, our data establish the contribution of lysosomal and mitochondrial crosstalk in Niemann-Pick diseases and underscore the role of sphingosine-1-phosphate signaling in modulating the pathogenesis of Niemann-Pick disease. 
Importantly, this work also provides the first line of evidence of encephalic involvement in Pompe's disease pathology. In Pompe's disease, mtDNA defects were the most predominant mitochondrial phenotype, although increased glycolysis also suggested reduced mitochondrial function. Our data established that impaired iron homeostasis due to lysosomal malfunction was the main culprit of mtDNA defects in Pompe's disease. In addition, mtDNA defects are associated with prevalent phenotypes of LSDs. Consistent with this, it was demonstrated that mtDNA defects promoted pro-inflammatory signaling via TLR9-mediated induction of the innate immune response, which subsequently resulted in gliosis in the cortex. Remarkably, the mechanisms elucidated in this study were valid in vivo since modulating iron levels was sufficient to reverse phenotypes of mtDNA defects and dampen the activated innate immunity in Pompe's disease. This study provides evidence that supports modulation of Pompe's disease pathology through dietary iron supplementation by reversing mitochondrial defects. Iron is a safe and prescribed dietary supplement in humans, which based on our findings, might be beneficial to Pompe's disease patients.

\subsection{Perspectives}

As with most scientific endeavors, this work has contributed to broadening our knowledge and understanding of the concepts of organelle interdependence, but more importantly, it raises a wealth of new questions to be addressed in subsequent studies. In line with this, it would be of utmost interest to explore the KLF2-ETV1-NRF1 network of transcriptional regulation of mitochondrial biogenesis in the context of other lysosomal malfunction states. It must be emphasized that signaling pathways between organelles go beyond transcriptional networks and these signaling routes require further investigation to enhance our understanding. The concept of organelle interplay is increasingly becoming clearer; however, its role in the pathogenesis of various diseases is not yet appreciated. Our evidence of CNS involvement in Pompe's disease is interesting since it presents a slower progression of the disease, whose molecular mechanisms we hope to investigate. mtDNA copy number loss has been proposed as a biomarker for neurodegenerative diseases, suggesting that it is worth investigating the mechanisms of mtDNA maintenance in the CNS.

Although this work was primarily aimed at understanding some of the mechanisms of crosstalk between lysosomes and mitochondria, the findings of the study delineated mechanisms that underpin human disease. To this end, the therapeutic avenues that were elucidated in this study warrant further exploration to understand their potential in protecting against diseases. 
Specifically, our findings establish that Pompe's disease shares some common features with other neurodegenerative diseases (e.g., Parkinson's disease), including mtDNA defects and impaired iron homeostasis. It would be interesting to know whether the same mechanism mediates the mtDNA defects in neurodegenerative disorders, and whether modulating iron levels could be beneficial.

This work does not exhaust the subject of organelle crosstalk. We have only elucidated some mechanisms of communication between lysosomes and mitochondria. However, mitochondria are known to contact other organelles, including the ER and peroxisomes, physically or via signaling pathways. For example, the mitochondria-ER contact sites have been the subject of intense research. It is particularly interesting to understand whether mitochondrial crosstalk with ER influences lysosomal function, and the mechanisms of communication between lysosomes and mitochondria. The interplay between mitochondria and peroxisomes has partly been established. These organelles are known to share important metabolic functions, as well as proteins that regulate their function. It is particularly thought-provoking to know whether a functional interdependent network exists between lysosomes, mitochondria and peroxisomes. Understanding how metabolic cues from organelle crosstalk are integrated by the nucleus in driving cellular functions through gene expression would be especially interesting.

Finally, understanding nature has been the ultimate quest of scientific endeavors. Despite considerable efforts along these lines, nature continually outpaces science and leaves us always with a wealth of questions to be addressed. For instance, eukaryotes are thought to have acquired membrane-bound compartments more than a billion years ago. Although there is an enormous wealth of knowledge on the basic role that these organelles play in ensuring cell survival, and the cooperation between multiple organelles in the execution of some cellular functions, our understanding of the mechanisms that regulate organelle function and interplay are still limited. It is my hope that this work, which sought to uncover some of the mechanisms of interdependence between lysosomes and mitochondria, has contributed, though insignificant, to our understanding of the functions of these subcellular compartments. 


\section{Bibliography}

Alam, M.S., Getz, M., Safeukui, I., Yi, S., Tamez, P., Shin, J., Velázquez, P., and Haldar, K. (2012). Genomic Expression Analyses Reveal Lysosomal, Innate Immunity Proteins, as Disease Correlates in Murine Models of a Lysosomal Storage Disorder. PLoS One 7.

Alberts-Grill, N., Engelbertsen, D., Bu, D., Foks, A., Grabie, N., Herter, J.M., Kuperwaser, F., Chen, T., Destefano, G., Jarolim, P., et al. (2016). Dendritic cell KLF2 expression regulates T cell activation and proatherogenic immune responses. J. Immunol. 197, 4651-4662.

Anderson, S., Bankier, A.T., Barrell, B.G., de Bruijn, M.H., Coulson, A.R., Drouin, J., Eperon, I.C., Nierlich, D.P., Roe, B.A., Sanger, F., et al. (1981). Sequence and organization of the human mitochondrial genome. Nature 290, 457-465.

Arsenijevic, D., Onuma, H., Pecqueur, C., Raimbault, S., Manning, B.S., Miroux, B., Couplan, E., Alves-Guerra, M.C., Goubern, M., Surwit, R., et al. (2000). Disruption of the uncoupling protein-2 gene in mice reveals a role in immunity and reactive oxygen species production. Nat. Genet. 26, 435-439.

Baena, E., Shao, Z., Linn, D.E., Glass, K., Hamblen, M.J., Fujiwara, Y., Kim, J., Nguyen, M., Zhang, X., Godinho, F.J., et al. (2013). ETV1 directs androgen metabolism and confers aggressive prostate cancer in targeted mice and patients. Genes Dev. 27, 683-698.

Bai, A., Hu, H., Yeung, M., and Chen, J. (2007). Kruppel-like factor 2 controls T cell trafficking by activating L-selectin (CD62L) and sphingosine-1-phosphate receptor 1 transcription. J. Immunol. 178, 7632-7639.

Baixauli, F., Acín-Pérez, R., Villarroya-Beltrí, C., Mazzeo, C., Nuñez-Andrade, N., GabandéRodriguez, E., Ledesma, M.D., Blázquez, A., Martin, M.A., Falcón-Pérez, J.M., et al. (2015). Mitochondrial respiration controls lysosomal function during inflammatory t cell responses. Cell Metab. 22, 485-498.

Bano, D., Young, K.W., Guerin, C.J., LeFeuvre, R., Rothwell, N.J., Naldini, L., Rizzuto, R., Carafoli, E., and Nicotera, P. (2005). Cleavage of the plasma membrane $\mathrm{Na}+\mathrm{Ca}+$ exchanger in excitotoxicity. Cell 120, 275-285.

Barbalat, R., Ewald, S.E., Mouchess, M.L., and Barton, G.M. (2011). Nucleic Acid Recognition by the Innate Immune System. Annu. Rev. Immunol. 29, 185-214.

Baulies, A., Ribas, V., Núñez, S., Torres, S., Alarcón-Vila, C., Martínez, L., Suda, J., Ybanez, M.D., Kaplowitz, N., García-Ruiz, C., et al. (2016). Lysosomal Cholesterol Accumulation Sensitizes To Acetaminophen Hepatotoxicity by Impairing Mitophagy. Sci. Rep. 5, 18017.

Bell, E.L., Klimova, T.A., Eisenbart, J., Moraes, C.T., Murphy, M.P., Budinger, G.R.S., and Chandel, N.S. (2007). The Qo site of the mitochondrial complex III is required for the transduction of hypoxic signaling via reactive oxygen species production. J. Cell Biol. 177, 1029-1036.

Berk, A.J., and Clayton, D.A. (1974). Mechanism of mitochondrial DNA replication in mouse L-cells: Asynchronous replication of strands, segregation of circular daughter molecules, aspects of topology and turnover of an initiation sequence. J. Mol. Biol. 86, 801-824.

Biasiotto, G., Di Lorenzo, D., Archetti, S., and Zanella, I. (2016). Iron and Neurodegeneration: Is Ferritinophagy the Link? Mol. Neurobiol. 53, 5542-5574. 
Bogorad, L. (1975). Evolution of Organelles and Eukaryotic Genomes. Science (80-. ). 188, 891-898.

Bosch, M.E., and Kielian, T. (2015). Neuroinflammatory paradigms in lysosomal storage diseases. Front. Neurosci. 9, 1-11.

Bourdon, A., Minai, L., Serre, V., Jais, J.-P., Sarzi, E., Aubert, S., Chrétien, D., de Lonlay, P., Paquis-Flucklinger, V., Arakawa, H., et al. (2007). Mutation of RRM2B, encoding p53controlled ribonucleotide reductase (p53R2), causes severe mitochondrial DNA depletion. Nat. Genet. 39, 776-780.

Brown, T.A., Tkachuk, A.N., Shtengel, G., Kopek, B.G., Bogenhagen, D.F., Hess, H.F., and Clayton, D.A. (2011). Superresolution Fluorescence Imaging of Mitochondrial Nucleoids Reveals Their Spatial Range, Limits, and Membrane Interaction. Mol. Cell. Biol. 31, 49945010.

Brozzi, A., Urbanelli, L., Germain, P.L., Magini, A., and Emiliani, C. (2013). hLGDB: A database of human lysosomal genes and their regulation. Database 2013, 1-6.

Bultron, G., Kacena, K., and Pearson, D. (2010). The risk of Parkinson's disease in type 1 Gaucher disease. J. Inherit Metab. Dis. 33, 167-173.

Burbulla, L.F., Song, P., Mazzulli, J.R., Zampese, E., Wong, Y.C., Jeon, S., Santos, D.P., Blanz, J., Obermaier, C.D., Strojny, C., et al. (2017). Dopamine oxidation mediates mitochondrial and lysosomal dysfunction in Parkinson's disease. Science (80-. ). 9080, 1-12.

Calvo, S.E., Clauser, K.R., and Mootha, V.K. (2016). MitoCarta2.0: An updated inventory of mammalian mitochondrial proteins. Nucleic Acids Res. 44, D1251-D1257.

Cantalupo, G., Alifano, P., Roberti, V., Bruni, C.B., and Bucci, C. (2001). Rab-interacting lysosomal protein (RILP): The Rab7 effector required for transport to lysosomes. EMBO J. 20, 683-693.

Cárdenas, C., Miller, R.A., Smith, I., Bui, T., Molgó, J., Müller, M., Vais, H., Cheung, K.H., Yang, J., Parker, I., et al. (2010). Essential Regulation of Cell Bioenergetics by Constitutive InsP3 Receptor Ca2+ Transfer to Mitochondria. Cell 142, 270-283.

Castellano, B.M., Thelen, A.M., Moldavski, O., Feltes, M., van der Welle, R.E.N., MydockMcGrane, L., Jiang, X., van Eijkeren, R.J., Davis, O.B., Louie, S.M., et al. (2017). Lysosomal cholesterol activates mTORC1 via an SLC38A9-Niemann-Pick C1 signaling complex.

Science (80-. ). 355, 1306-1311.

Castillo, J.J., Jelinek, D., Wei, H., Gannon, N.P., Vaughan, R.A., Horwood, L.J., Meaney, F.J., Garcia-Smith, R., Trujillo, K.A., Heidenreich, R.A., et al. (2017). The Niemann-Pick C1 gene interacts with a high-fat diet to promote weight gain through differential regulation of central energy metabolism pathways. Am. J. Physiol. - Endocrinol. Metab. ajpendo.00369.2016.

Chacinska, A., Koehler, C.M., Milenkovic, D., Lithgow, T., and Pfanner, N. (2009). Importing Mitochondrial Proteins: Machineries and Mechanisms. Cell 138, 628-644.

Chan, D.C. (2012). Fusion and Fission: Interlinked Processes Critical for Mitochondrial Health. Annu. Rev. Genet. 46, 265-287.

Chang, J., Lee, S., and Blackstone, C. (2014). Spastic paraplegia proteins spastizin and spatacsin mediate autophagic lysosome reformation. J. Clin. Invest. 124, 5249-5262. 
Chantranupong, L., Wolfson, R.L., and Sabatini, D.M. (2015). Nutrient-sensing mechanisms across evolution. Cell 161, 67-83.

Chatzi, A., Manganas, P., and Tokatlidis, K. (2016). Oxidative folding in the mitochondrial intermembrane space: A regulated process important for cell physiology and disease.

Biochim. Biophys. Acta - Mol. Cell Res. 1863, 1298-1306.

Chauhan, S., Goodwin, J.G., Chauhan, S., Manyam, G., Wang, J., Kamat, A.M., and Boyd, D.D. (2013). ZKSCAN3 Is a Master Transcriptional Repressor of Autophagy. Mol. Cell 50, 16-28.

Chen, Y., and Yu, L. (2017). Recent progress in autophagic lysosome reformation. Traffic 18, 358-361.

Chen, H., Detmer, S.A., Ewald, A.J., Griffin, E.E., Fraser, S.E., and Chan, D.C. (2003). Mitofusins Mfn1 and Mfn2 coordinately regulate mitochondrial fusion and are essential for embryonic development. J. Cell Biol. 160, 189-200.

Chi, P., Chen, Y., Zhang, L., Guo, X., Wongvipat, J., Shamu, T., Fletcher, J.A., Dewell, S., Maki, R.G., Zheng, D., et al. (2010). ETV1 is a lineage survival factor that cooperates with KIT in gastrointestinal stromal tumours. Nature 467, 849-853.

Chin, R.M., Fu, X., Pai, M.Y., Vergnes, L., Hwang, H., Deng, G., Diep, S., Lomenick, B., Meli, V.S., Monsalve, G.C., et al. (2014). The metabolite $\alpha$-ketoglutarate extends lifespan by inhibiting ATP synthase and TOR. Nature.

Christensen, K. a, Myers, J.T., and Swanson, J. a (2002). pH-dependent regulation of lysosomal calcium in macrophages. J. Cell Sci. 115, 599-607.

Colombini, M. (1980). Pore size and properties of channels from mitochondria isolated fromNeurospora crassa. J. Membr. Biol. 53, 79-84.

Cotney, J., Wang, Z., and Shadel, G.S. (2007). Relative abundance of the human mitochondrial transcription system and distinct roles for h-mtTFB1 and h-mtTFB2 in mitochondrial biogenesis and gene expression. Nucleic Acids Res. 35, 4042-4054.

Cribbs, J.T., and Strack, S. (2007). Reversible phosphorylation of Drp1 by cyclic AMPdependent protein kinase and calcineurin regulates mitochondrial fission and cell death. EMBO Rep. 8, 939-944.

D’Aurelio, M., Pallotti, F., Barrientos, A., Gajewski, C.D., Kwong, J.Q., Bruno, C., Beal, M.F., and Manfredi, G. (2001). In Vivo Regulation of Oxidative Phosphorylation in Cells Harboring a Stop-codon Mutation in Mitochondrial DNA-encoded Cytochrome c Oxidase Subunit I. J. Biol. Chem. 276, 46925-46932.

Das, M., Lu, J., Joseph, M., Aggarwal, R., Kanji, S., McMichael, B.K., Lee, B.S., Agarwal, S., Ray-Chaudhury, a., Iwenofu, O.H., et al. (2012). Kruppel-Like Factor 2 (KLF2) Regulates Monocyte Differentiation and Functions in mBSA and IL-1 $\beta$-Induced Arthritis. Curr. Mol. Med. 12, 113-125.

Davies, J.P., and loannou, Y.A. (2000). Topological analysis of Niemann-Pick C1 protein reveals that the membrane orientation of the putative sterol-sensing domain is identical to those of 3-hydroxy-3-methylglutaryl-CoA reductase and sterol regulatory element binding protein cleavage-activating. J. Biol. Chem. 275, 24367-24374.

Dekker, P.J.T., Martin, F., Maarse, A.C., Bömer, U., Müller, H., Guiard, B., Meijer, M., 
Rassow, J., and Pfanner, N. (1997). The Tim core complex defines the number of mitochondrial translocation contact sites and can hold arrested preproteins in the absence of matrix Hsp70-Tim44. EMBO J. 16, 5408-5419.

Demers-Lamarche, J., Guillebaud, G., Tlili, M., Todkar, K., B??langer, N., Grondin, M., P'Nguyen, A., Michel, J., and Germain, M. (2016). Loss of mitochondrial function impairs Lysosomes. J. Biol. Chem. 291, 10263-10276.

DeRuisseau, L.R., Fuller, D.D., Qiu, K., DeRuisseau, K.C., Donnelly Jr., W.H., Mah, C., Reier, P.J., and Byrne, B.J. (2009). Neural deficits contribute to respiratory insufficiency in Pompe disease. Proc Natl Acad Sci U S A 106, 9419-9424.

DiMauro, S. (2004). Mitochondrial diseases. Biochim. Biophys. Acta - Bioenerg. 1658, 80-88.

DiMauro, S., and Davidzon, G. (2005). Mitochondrial DNA and disease. Ann. Med. 37, 222232.

Diogo, C. V., Yambire, K.F., Fernández Mosquera, L., Branco F., T., and Raimundo, N. (2017). Mitochondrial adventures at the organelle society. Biochem. Biophys. Res. Commun. $1-7$.

Doddaballapur, A., Michalik, K.M., Manavski, Y., Lucas, T., Houtkooper, R.H., You, X., Chen, W., Zeiher, A.M., Potente, M., Dimmeler, S., et al. (2015). Laminar shear stress inhibits endothelial cell metabolism via KLF2-mediated repression of PFKFB3. Arterioscler. Thromb. Vasc. Biol. 35, 137-145.

Douglas, A.E. (2014). Symbiosis as a general principle in eukaryotic evolution. Cold Spring Harb. Perspect. Biol. 6, 1-14.

Doulias, P.T., Christoforidis, S., Brunk, U.T., and Galaris, D. (2003). Endosomal and lysosomal effects of desferrioxamine: Protection of HeLa cells from hydrogen peroxideinduced DNA damage and induction of cell-cycle arrest. Free Radic. Biol. Med. 35, 719-728.

Dugas, J.C., Tai, Y.C., Speed, T.P., Ngai, J., and Barres, B.A. (2006). Functional genomic analysis of oligodendrocyte differentiation. J. Neurosci. 26, 10967-10983.

De Duve, C., Pressman, B.C., Gianetto, R., Wattiaux, R., and Appelmans, F. (1955). Tissue fractionation studies. 6 . Intracellular distribution patterns of enzymes in rat-liver tissue. Biochem. J. 60, 604-617.

El-Hattab, A.W., Craigen, W.J., and Scaglia, F. (2017). Mitochondrial DNA maintenance defects. Biochim. Biophys. Acta - Mol. Basis Dis. 1863, 1539-1555.

Elbaz-Alon, Y., Eisenberg-Bord, M., Shinder, V., Stiller, S.B., Shimoni, E., Wiedemann, N., Geiger, T., and Schuldiner, M. (2015). Lam6 Regulates the Extent of Contacts between Organelles. Cell Rep. 12, 7-14.

Evans, M.J., and Scarpullas, C. (1989). Interaction of Nuclear Factors with Multiple Sites in the Somatic Cytochrome c Promoter. J Biol Chem 264, 14361-14368.

Ewald, S.E., Lee, B.L., Lau, L., Wickliffe, K.E., Shi, G.-P., Chapman, H.A., and Barton, G.M. (2008). The ectodomain of Toll-like receptor 9 is cleaved to generate a functional receptor. Nature 456, 658-662.

Facci, L., Barbierato, M., Marinelli, C., Argentini, C., Skaper, S.D., and Giusti, P. (2014). TollLike Receptors 2, -3 and -4 Prime Microglia but not Astrocytes Across Central Nervous 
System Regions for ATP-Dependent Interleukin-1 $\beta$ Release. Sci. Rep. 4, 6824.

Falk, D.J., Todd, A.G., Lee, S., Soustek, M.S., Elmallah, M.K., Fuller, D.D., Notterpek, L., and Byrne, B.J. (2015). Peripheral nerve and neuromuscular junction pathology in pompe disease. Hum. Mol. Genet. 24, 625-636.

Fan, M., Sidhu, R., Fujiwara, H., Tortelli, B., Zhang, J., Davidson, C., Walkley, S.U., Bagel, J.H., Vite, C., Yanjanin, N.M., et al. (2013). Identification of Niemann-Pick C1 disease biomarkers through sphingolipid profiling. J. Lipid Res. 54, 2800-2814.

Feng, R., Desbordes, S.C., Xie, H., Tillo, E.S., Pixley, F., Stanley, E.R., and Graf, T. (2008). PU.1 and C/EBPalpha/beta convert fibroblasts into macrophage-like cells. Proc. Natl. Acad. Sci. U. S. A. 105, 6057-6062.

Fernández-Mosquera, L., Diogo, C. V, Yambire, K.F., Santos, G.L., Luna Sánchez, M., Bénit, P., Rustin, P., Lopez, L.C., Milosevic, I., and Raimundo, N. (2017). Acute and chronic mitochondrial respiratory chain deficiency differentially regulate lysosomal biogenesis. Sci. Rep. 7, 1-11.

Di Fiore, P.P., and von Zastrow, M. (2014). Endocytosis, signaling, and beyond. Cold Spring Harb. Perspect. Biol. 6, 1-14.

Forgac, M. (2007). Vacuolar ATPases: rotary proton pumps in physiology and pathophysiology. Nat. Rev. Mol. Cell Biol. 8, 917-929.

Friedland, N., Liou, H.-L., Lobel, P., and Stock, A.M. (2003). Structure of a cholesterol-binding protein deficient in Niemann-Pick type C2 disease. Proc. Natl. Acad. Sci. U. S. A. 100, 25122517.

Gandre-Babbe, S., and van der Bliek, A.M. (2008). The novel tail-anchored membrane protein Mff controls mitochondrial and peroxisomal fission in mammalian cells. Mol. Biol. Cell 19, 2402-2412.

Gao, Y., Chen, Y., Zhan, S., Zhang, W., Xiong, F., and Ge, W. (2017). Comprehensive proteome analysis of lysosomes reveals the diverse function of macrophages in immune responses. Oncotarget $8,7420-7440$.

German, D.C., Liang, C.L., Song, T., Yazdani, U., Xie, C., and Dietschy, J.M. (2002). Neurodegeneration in the Niemann-Pick C mouse: Glial involvement. Neuroscience 109, 437-450.

Gilchrist, A., Au, C.E., Hiding, J., Bell, A.W., Fernandez-Rodriguez, J., Lesimple, S., Nagaya, H., Roy, L., Gosline, S.J.C., Hallett, M., et al. (2006). Quantitative Proteomics Analysis of the Secretory Pathway. Cell 127, 1265-1281.

Gray, M.W., Burger, G., and Lang, B.F. (1999). Mitochondrial Evolution. Science (80-. ). 283, $1-16$.

Grishchuk, Y., Pen, K.A., Coblentz, J., King, V.E., Humphrey, D.M., Wang, S.L., Kiselyov, K.I., Slaugenhaupt, S.A., Y., G., K.A., P., et al. (2015). Impaired myelination and reduced brain ferric iron in the mouse model of mucolipidosis IV. DMM Dis. Model. Mech. 8, 15911601.

Gui, Y.X., Xu, Z.P., Lv, W., Zhao, J.J., and Hu, X.Y. (2015). Evidence for polymerase gamma, POLG1 variation in reduced mitochondrial DNA copy number in Parkinson's disease. Park. Relat. Disord. 21, 282-286. 
Guittet, O., Håkansson, P., Voevodskaya, N., Fridd, S., Gräslund, A., Arakawa, H., Nakamura, Y., and Thelander, L. (2001). Mammalian p53R2 Protein Forms an Active Ribonucleotide Reductase in Vitro with the R1 Protein, Which Is Expressed Both in Resting Cells in Response to DNA Damage and in Proliferating Cells. J. Biol. Chem. 276, 4064740651.

Gulick, T., Cresci, S., Caira, T., Moore, D.D., and Kelly, D.P. (1994). The peroxisome proliferator-activated receptor regulates mitochondrial fatty acid oxidative enzyme gene expression. Proc. Natl. Acad. Sci. U. S. A. 91, 11012-11016.

Gustafsson, C.M., Falkenberg, M., and Larsson, N.-G. (2016). Maintenance and Expression of Mammalian Mitochondrial DNA. Annu. Rev. Biochem. 85, 133-160.

Hagen, N., Hans, M., Hartmann, D., Swandulla, D., and van Echten-Deckert, G. (2011). Sphingosine-1-phosphate links glycosphingolipid metabolism to neurodegeneration via a calpain-mediated mechanism. Cell Death Differ. 18, 1356-1365.

Hamanaka, R.B., and Chandel, N.S. (2010). Mitochondrial reactive oxygen species regulate cellular signaling and dictate biological outcomes. Trends Biochem. Sci. 35, 505-513.

Hell, K., Herrmann, J.M., Pratje, E., Neupert, W., and Stuart, R.A. (1998). Oxa1p, an essential component of the $\mathrm{N}$-tail protein export machinery in mitochondria. Proc. Natl. Acad. Sci. 95, 2250-2255.

Herrmann, J.M., and Riemer, J. (2010). The intermembrane space of mitochondria. Antioxid. Redox Signal. 13, 1341-1358.

Holt, I.J., Lorimer, H.E., and Jacobs, H.T. (2000). Coupled Leading- and Lagging-Strand Synthesis of Mammalian Mitochondrial DNA. Cell 100, 515-524.

Hsing, L.C., and Rudensky, A.Y. (2005). The lysosomal cysteine proteases in MHC class II antigen presentation. Immunol. Rev. 207, 229-241.

Irizarry, R.A., Bolstad, B.M., Collin, F., Cope, L.M., Hobbs, B., and Speed, T.P. (2003). Summaries of Affymetrix GeneChip probe level data. Nucleic Acids Res. 31, e15.

Janknecht, R. (1996). Analysis of the ERK-stimulated ETS transcription factor ER81. Mol. Cell. Biol. 16, 1550-1556.

Janknecht, R. (2003). Regulation of the ER81 transcription factor and its coactivators by mitogen- and stress-activated protein kinase 1 (MSK1). Oncogene 22, 746-755.

Jenkins, R.W., Idkowiak-Baldys, J., Simbari, F., Canals, D., Roddy, P., Riner, C.D., Clarke, C.J., and Hannun, Y.A. (2011). A novel mechanism of lysosomal acid sphingomyelinase maturation: Requirement for carboxyl-terminal proteolytic processing. J. Biol. Chem. 286, 3777-3788.

Jouaville, L.S., Pinton, P., Bastianutto, C., Rutter, G. a, and Rizzuto, R. (1999). Regulation of mitochondrial ATP synthesis by calcium: evidence for a long-term metabolic priming. Proc. Natl. Acad. Sci. U. S. A. 96, 13807-13812.

Karageorgos, L.E., Isaac, E.L., Brooks, D. a, Ravenscroft, E.M., Davey, R., Hopwood, J.J., and Meikle, P.J. (1997). Lysosomal biogenesis in lysosomal storage disorders. Exp. Cell Res. 234, 85-97.

Kaufman, B.A., Durisic, N., Mativetsky, J.M., Constantino, S., Hancock, M.A., Grutter, P., and 
Shoubridge, E.A. (2007). The Mitochondrial Transcription Factor TFAM Coordinates the Assembly of Multiple DNA Molecules into Nucleoid-like Structures. Mol. Biol. Cell 18, 32253236.

Kaushik, S., and Cuervo, A.M. (2012). Chaperone-mediated autophagy: A unique way to enter the lysosome world. Trends Cell Biol. 22, 407-417.

Kawai, T., and Akira, S. (2010). The role of pattern-recognition receptors in innate immunity: update on Toll-like receptors. Nat. Immunol. 11, 373-384.

Kawai, T., Takahashi, K., Sato, S., Coban, C., Kumar, H., Kato, H., Ishii, K.J., Takeuchi, O., and Akira, S. (2005). IPS-1, an adaptor triggering RIG-I- and Mda5-mediated type I interferon induction. Nat. Immunol. 6, 981-988.

Kelly, D.P., and Scarpulla, R.C. (2004). Transcriptional regulatory circuits controlling mitochondrial biogenesis and function. Genes Dev. 357-368.

Keogh, M.J., and Chinnery, P.F. (2015). Mitochondrial DNA mutations in neurodegeneration. Biochim. Biophys. Acta 1847, 1401-1411.

Kizaki, T., Suzuki, K., Hitomi, Y., Taniguchi, N., Saitoh, D., Watanabe, K., Onoé, K., Day, N.K., Good, R.A., and Ohno, H. (2002). Uncoupling protein 2 plays an important role in nitric oxide production of lipopolysaccharide-stimulated macrophages. Proc. Natl. Acad. Sci. U. S. A. 99, 9392-9397.

Klionsky, D.J., Cregg, J.M., Dunn, W.A., Emr, S.D., Sakai, Y., Sandoval, I. V., Sibirny, A., Subramani, S., Thumm, M., Veenhuis, M., et al. (2003). A unified nomenclature for yeast autophagy-related genes. Dev. Cell 5, 539-545.

Klumperman, J., and Raposo, G. (2014). The complex ultrastructure of the endolysosomal system. Cold Spring Harb. Perspect. Biol. 6.

Korhonen, J.A., Pham, X.H., Pellegrini, M., and Falkenberg, M. (2004). Reconstitution of a minimal mtDNA replisome in vitro. EMBO J. 23, 2423-2429.

Kornfeld, S., and Mellman, I. (1989). The biogenesis of I ysosomes. Annu. Rev. Cell Biol 483-525.

Koshiba, T. (2004). Structural Basis of Mitochondrial Tethering by Mitofusin Complexes. Science (80-. ). 305, 858-862.

Krauss, S. (2001). Mitochondria : Structure and Role in Respiration. Life Sci. 1-6.

Krebs, H.A., and Johnson, W.A. (1937). The role of citric acid in intermediary metabolism in animal tissue. Enzymologia 4, 148-156.

Kukat, C., Wurm, C.A., Spahr, H., Falkenberg, M., Larsson, N.-G., and Jakobs, S. (2011).

Super-resolution microscopy reveals that mammalian mitochondrial nucleoids have a uniform size and frequently contain a single copy of mtDNA. Proc. Natl. Acad. Sci. 108, 1353413539.

Kurz, T., Terman, A., Gustafsson, B., and Brunk, U.T. (2008). Lysosomes in iron metabolism, ageing and apoptosis. Histochem. Cell Biol. 129, 389-406.

Lachmann, R.H., Te Vruchte, D., Lloyd-Evans, E., Reinkensmeier, G., Sillence, D.J., Fernandez-Guillen, L., Dwek, R.A., Butters, T.D., Cox, T.M., and Platt, F.M. (2004).

Treatment with miglustat reverses the lipid-trafficking defect in Niemann-Pick disease type C. 
Neurobiol. Dis. 16, 654-658.

Lane, N., and Martin, W. (2010). The energetics of genome complexity. Nature 467, 929-934.

Lane, D.J.R., Merlot, A.M., Huang, M.L.H., Bae, D.H., Jansson, P.J., Sahni, S., Kalinowski, D.S., and Richardson, D.R. (2015). Cellular iron uptake, trafficking and metabolism: Key molecules and mechanisms and their roles in disease. Biochim. Biophys. Acta - Mol. Cell Res. 1853, 1130-1144.

Laplante, M., and Sabatini, D.M. (2012). MTOR signaling in growth control and disease. Cell 149, 274-293.

Larsson, N.G., Wang, J., Wilhelmsson, H., Oldfors, a, Rustin, P., Lewandoski, M., Barsh, G.S., and Clayton, D. a (1998). Mitochondrial transcription factor A is necessary for mtDNA maintenance and embryogenesis in mice. Nat. Genet. 18, 231-236.

LeBleu, V.S., O'Connell, J.T., Gonzalez Herrera, K.N., Wikman, H., Pantel, K., Haigis, M.C., de Carvalho, F.M., Damascena, A., Domingos Chinen, L.T., Rocha, R.M., et al. (2014). PGC$1 \alpha$ mediates mitochondrial biogenesis and oxidative phosphorylation in cancer cells to promote metastasis. Nat. Cell Biol. 16, 992-1003.

Lewis, M.R., and Lewis, W.H. (1914). Mitochondria in tissue culture. Science (80-. ). 39, 330333.

Li, J.Y., Paragas, N., Ned, R.M., Qiu, A., Viltard, M., Leete, T., Drexler, I.R., Chen, X., SannaCherchi, S., Mohammed, F., et al. (2009). Scara5 Is a Ferritin Receptor Mediating Non-

Transferrin Iron Delivery. Dev. Cell 16, 35-46.

Li, X., Rydzewski, N., Hider, A., Zhang, X., Yang, J., Wang, W., Gao, Q., Cheng, X., and Xu, $\mathrm{H}$. (2016). A molecular mechanism to regulate lysosome motility for lysosome positioning and tubulation. Nat. Cell Biol. 18, 404-417.

Lim, J.A., Li, L., Kakhlon, O., Myerowitz, R., and Raben, N. (2015). Defects in calcium homeostasis and mitochondria can be reversed in Pompe disease. Autophagy 11, 385-402.

Lin, J., Handschin, C., and Spiegelman, B.M. (2005). Metabolic control through the PGC-1 family of transcription coactivators. Cell Metab. 1, 361-370.

Lipmann, F., Kaplan, N.O., Novelli, G.D., Tuttle, C.L., and Giurard, B.M. (1950). Isolation of Coenzyme A. J. Biol. Chem. 186, 235-243.

Lloyd-Evans, E., Morgan, A.J., He, X., Smith, D. a, Elliot-Smith, E., Sillence, D.J., Churchill, G.C., Schuchman, E.H., Galione, A., and Platt, F.M. (2008). Niemann-Pick disease type C1 is a sphingosine storage disease that causes deregulation of lysosomal calcium. Nat. Med. 14, 1247-1255.

Loson, O.C., Song, Z., Chen, H., and Chan, D.C. (2013). Fis1, Mff, MiD49, and MiD51 mediate Drp1 recruitment in mitochondrial fission. Mol. Biol. Cell 24, 659-667.

Ma, X.M., and Blenis, J. (2009). Molecular mechanisms of mTOR-mediated translational control. Nat. Rev. Mol. Cell Biol. 10, 307-318.

Manavski, Y., Abel, T., Hu, J., Kleinlützum, D., Buchholz, C.J., Belz, C., Augustin, H.G., Boon, R.A., and Dimmeler, S. (2017). Endothelial transcription factor KLF2 negatively regulates liver regeneration via induction of activin A. Proc. Natl. Acad. Sci. 114, 3993-3998.

Mancias, J.D., Wang, X., Gygi, S.P., Harper, J.W., and Kimmelman, A.C. (2014). Quantitative 
proteomics identifies NCOA4 as the cargo receptor mediating ferritinophagy. Nature 509, 105-109.

Marco Sardiello, Michela Palmieri, Alberto di Ronza, Diego Luis Medina, M.V., Vincenzo Alessandro Gennarino, Chiara Di Malta, Francesca Donaudy, V.E., and Roman S. Polishchuk, Sandro Banfi, Giancarlo Parenti, Elena Cattaneo, A.B. (2009). A Gene Network Regulating Lysosomal Biogenesis and Function. Science (80-. ). 325, 473-477.

Maroulakou, I.G., and Bowe, D.B. (2000). Expression and function of Ets transcription factors in mammalian development: a regulatory network. Oncogene 19,6432-6442.

Martina, J.A., Chen, Y., Gucek, M., and Puertollano, R. (2012). MTORC1 functions as a transcriptional regulator of autophagy by preventing nuclear transport of TFEB. Autophagy 8 , 903-914.

Martina, J.A., Diab, H.I., Lishu, L., Jeong-A, L., Patange, S., Raben, N., and Puertollano, R. (2014). The Nutrient-Responsive Transcription Factor TFE3 Promotes Autophagy, Lysosomal Biogenesis, and Clearance of Cellular Debris. Sci. Signal. 7, ra9-ra9.

Matak, P., Matak, A., Moustafa, S., Aryal, D.K., Benner, E.J., Wetsel, W., and Andrews, N.C. (2016). Disrupted iron homeostasis causes dopaminergic neurodegeneration in mice. Proc. Natl. Acad. Sci. 113, 3428-3435.

Matsushima, Y., Goto, Y., and Kaguni, L.S. (2010). Mitochondrial Lon protease regulates mitochondrial DNA copy number and transcription by selective degradation of mitochondrial transcription factor A ( TFAM ).

Medina, D.L., Di Paola, S., Peluso, I., Armani, A., De Stefani, D., Venditti, R., Montefusco, S., Scotto-Rosato, A., Prezioso, C., Forrester, A., et al. (2015). Lysosomal calcium signalling regulates autophagy through calcineurin and TFEB. Nat. Cell Biol. 17, 288-299.

Mendoza, A., Fang, V., Chen, C., Serasinghe, M., Verma, A., Muller, J., Chaluvadi, V.S., Dustin, M.L., Hla, T., Elemento, O., et al. (2017). Lymphatic endothelial S1P promotes mitochondrial function and survival in naive T cells. Nature 546, 158-161.

Meylan, E., Curran, J., Hofmann, K., Moradpour, D., Binder, M., Bartenschlager, R., and Tschopp, J. (2005). Cardif is an adaptor protein in the RIG-I antiviral pathway and is targeted by hepatitis C virus. Nature 437, 1167-1172.

Meyron-Holtz, E.G. (2004). Mammalian Tissue Oxygen Levels Modulate Iron-Regulatory Protein Activities in Vivo. Science (80-. ). 306, 2087-2090.

Mindell, J.A. (2012). Lysosomal acidification mechanisms. Annu Rev Physiol 74, 69-86.

Mitchell, P. (1961). Coupling of phosphorylation to electron and hydrogen transfer by a chemi-osmotic type of mechanism. Nature 191, 144-148.

Mizushima, N., and Komatsu, M. (2011). Autophagy: Renovation of cells and tissues. Cell $147,728-741$.

Mokranjac, D., and Neupert, W. (2005). Protein Import into Mitochondria. Biochem. Soc. Trans. 33, 1019-1023.

Morita, M., Gravel, S., Chénard, V., Sikstro, K., Zheng, L., Alain, T., Gandin, V., Avizonis, D., Arguello, M., Zakaria, C., et al. (2013). mTORC1 Controls Mitochondrial Activity and Biogenesis through 4E-BP-Dependent Translational Regulation. Cell Metab. 18, 698-711. 
Morrish, F., Giedt, C., and Hockenbery, D. (2003). c-MYC apoptotic function is mediated by NRF-1 target genes. Genes Dev. 17, 240-255.

Müller, M., Mentel, M., van Hellemond, J.J., Henze, K., Woehle, C., Gould, S.B., Yu, R.-Y., van der Giezen, M., Tielens, A.G.M., and Martin, W.F. (2012). Biochemistry and evolution of anaerobic energy metabolism in eukaryotes. Microbiol. Mol. Biol. Rev. 76, 444-495.

Nass, S., and Nass, M.M.K. (1963). Intramitochondrial fibers with DNA characteristics. J. Cell Biol. 19, 613-629.

Nezich, C.L., Wang, C., Fogel, A.I., and Youle, R.J. (2015). MiT/TFE transcription factors are activated during mitophagy downstream of Parkin and Atg5. J. Cell Biol. 210, 435-450.

Nikkanen, J., Forsström, S., Euro, L., Paetau, I., Kohnz, R.A., Wang, L., Chilov, D., Viinamäki, J., Roivainen, A., Marjamäki, P., et al. (2016). Mitochondrial DNA Replication Defects Disturb Cellular dNTP Pools and Remodel One-Carbon Metabolism. Cell Metab. 23, 635-648.

Nilsson, R., Jain, M., Madhusudhan, N., Sheppard, Nina Gustafsson 1, Strittmatter, L., Kampf, C., Huang, J., Asplund, Anna, A., and Mootha, V.K. (2014). Metabolic enzyme expression highlights a key role for MTHFD2 and the mitochondrial folate pathway in cancer. Nat. Commun. 5, 3128.

Nunnari, J., and Suomalainen, A. (2012). Mitochondria: In sickness and in health. Cell 148, 1145-1159.

O'Neill, L.A.J., Golenbock, D., and Bowie, A.G. (2013). The history of Toll-like receptors redefining innate immunity. Nat. Rev. Immunol. 13, 453-460.

Ohguchi, H., Hideshima, T., Bhasin, M.K., Gorgun, G.T., Santo, L., Cea, M., Samur, M.K., Mimura, N., Suzuki, R., Tai, Y.-T., et al. (2016). The KDM3A-KLF2-IRF4 axis maintains myeloma cell survival. Nat. Commun. 7, 10258.

Oka, T., Hikoso, S., Yamaguchi, O., Taneike, M., Takeda, T., Tamai, T., Oyabu, J., Murakawa, T., Nakayama, H., Nishida, K., et al. (2012). Mitochondrial \{DNA\} that escapes from autophagy causes inflammation and heart failure. Nature 485, 251-255.

Ordonez, M.P. (2012). Defective mitophagy in human Niemann-Pick type C1 neurons is due to abnormal autophagy activation. Autophagy 8, 1157-1158.

Osellame, L.D., and Duchen, M.R. (2014). Quality control gone wrong: Mitochondria, lysosomal storage disorders and neurodegeneration. Br. J. Pharmacol. 171, 1958-1972.

Osellame, L.D., Rahim, A. a, Hargreaves, I.P., Gegg, M.E., Richard-Londt, A., Brandner, S., Waddington, S.N., Schapira, A.H. V, and Duchen, M.R. (2013). Mitochondria and quality control defects in a mouse model of Gaucher disease--links to Parkinson's disease. Cell Metab. 17, 941-953.

Pagliarini, D.J., Calvo, S.E., Chang, B., Sheth, S.A., Vafai, S.B., Ong, S.E., Walford, G.A., Sugiana, C., Boneh, A., Chen, W.K., et al. (2008). A Mitochondrial Protein Compendium Elucidates Complex I Disease Biology. Cell 134, 112-123.

Palmer, C.S., Elgass, K.D., Parton, R.G., Osellame, L.D., Stojanovski, D., and Ryan, M.T. (2013). Adaptor proteins MiD49 and MiD51 can act independently of Mff and Fis1 in Drp1 recruitment and are specific for mitochondrial fission. J. Biol. Chem. 288, 27584-27593. 
Palmieri, M., Impey, S., Kang, H., di Ronza, A., Pelz, C., Sardiello, M., and Ballabio, A. (2011). Characterization of the CLEAR network reveals an integrated control of cellular clearance pathways. Hum. Mol. Genet. 20, 3852-3866.

Palmieri, M., Pal, R., Nelvagal, H.R., Lotfi, P., Stinnett, G.R., Seymour, M.L., Chaudhury, A., Bajaj, L., Bondar, V. V., Bremner, L., et al. (2017). mTORC1-independent TFEB activation via Akt inhibition promotes cellular clearance in neurodegenerative storage diseases. Nat. Commun. 8, 14338.

Paschen, S. a., Waizenegger, T., Stan, T., Preuss, M., Cyrklaff, M., Hell, K., Rapaport, D., and Neupert, W. (2003). Evolutionary conservation of biogenesis of b -barrel membrane proteins. Nature 426, 862-866.

Patten, D.A., Wong, J., Khacho, M., Soubannier, V., Mailloux, R.J., Pilon-larose, K., Maclaurin, J.G., Park, D.S., Mcbride, H.M., Trinkle-mulcahy, L., et al. (2014). OPA1dependent cristae modulation is essential for cellular adaptation to metabolic demand. EMBO J. 33, 2676-2691.

Patterson, M.C., and Walkley, S.U. (2017). Niemann-Pick disease, type C and Roscoe Brady. Mol. Genet. Metab. 120, 34-37.

Patterson, M.C., Hendriksz, C.J., Walterfang, M., Sedel, F., Vanier, M.T., and Wijburg, F. (2012). Recommendations for the diagnosis and management of Niemann-Pick disease type C: An update. Mol. Genet. Metab. 106, 330-344.

Perera, R.M., and Zoncu, R. (2016). The Lysosome as a Regulatory Hub. Annu. Rev. Cell Dev. Biol. 32, annurev-cellbio-111315-125125.

Pernas, L., and Scorrano, L. (2016). Mito-Morphosis: Mitochondrial Fusion, Fission, and Cristae Remodeling as Key Mediators of Cellular Function. Annu. Rev. Physiol. 78, 505-531.

Persidsky, Y., Ghorpade, A., Rasmussen, J., Limoges, J., Liu, X.J., Stins, M., Fiala, M., Way, D., Kim, K.S., Witte, M.H., et al. (1999). Microglial and Astrocyte Chemokines Regulate Monocyte Migration through the Blood-Brain Barrier in Human Immunodeficiency Virus-1 Encephalitis. Am. J. Pathol. 155, 1599-1611.

Pickrell, A.M., and Youle, R.J. (2015). The roles of PINK1, Parkin, and mitochondrial fidelity in parkinson's disease. Neuron 85, 257-273.

Di Pietro, E., Wang, X.L., and MacKenzie, R.E. (2004). The expression of mitochondrial methylenetetrahydrofolate dehydrogenase-cyclohydrolase supports a role in rapid cell growth. Biochim. Biophys. Acta - Gen. Subj. 1674, 78-84.

Pinto, M., and Moraes, C.T. (2014). Biochimica et Biophysica Acta Mitochondrial genome changes and neurodegenerative diseases is. BBA - Mol. Basis Dis. 1842, 1198-1207.

Platt, F.M. (2014). Sphingolipid lysosomal storage disorders. Nature 510, 68-75.

Platt, F.M., Boland, B., and van der Spoel, A.C. (2012). Lysosomal storage disorders: The cellular impact of lysosomal dysfunction. J. Cell Biol. 199, 723-734.

Platt, N., Speak, A.O., Colaco, A., Gray, J., Smith, D.A., Williams, I.M., Wallom, K.L., and Platt, F.M. (2016). Immune dysfunction in Niemann-Pick disease type C. J. Neurochem. 136, 74-80.

van der Ploeg, A.T., and Reuser, A.J. (2008). Pompe's disease. Lancet 372, 1342-1353. 
Ploper, D., and De Robertis, E.M. (2015). The MITF family of transcription factors: Role in endolysosomal biogenesis, Wnt signaling, and oncogenesis. Pharmacol. Res. 99, 36-43.

Pollard, P.J., Brière, J.J., Alam, N.A., Barwell, J., Barclay, E., Wortham, N.C., Hunt, T., Mitchell, M., Olpin, S., Moat, S.J., et al. (2005). Accumulation of Krebs cycle intermediates and over-expression of HIF1 $\alpha$ in tumours which result from germline FH and SDH mutations. Hum. Mol. Genet. 14, 2231-2239.

Pontarin, G., Ferraro, P., Bee, L., Reichard, P., and Bianchi, V. (2012). Mammalian ribonucleotide reductase subunit $\mathrm{p53R2}$ is required for mitochondrial DNA replication and DNA repair in quiescent cells. Proc. Natl. Acad. Sci. U. S. A. 109, 13302-13307.

Poole, A.M., Gribaldo, S., Guy, L., Saw, J.H., Ettema, T.J.G., Eme, L., Sharpe, S.C., Brown, M.W., Irimia, M., and Roy, S.W. (2014). Eukaryotic Origins: How and When Was the Mitochondrion Acquired? Cold Spring Harb. Perspect. Biol.

Pu, J., Schindler, C., Jia, R., Jarnik, M., Backlund, P., and Bonifacino, J.S. (2015). BORC, a Multisubunit Complex that Regulates Lysosome Positioning. Dev. Cell 33, 176-188.

Puigserver, P., Wu, Z., Park, C.W., Graves, R., Wright, M., and Spiegelman, B.M. (1998). A cold-inducible coactivator of nuclear receptors linked to adaptive thermogenesis. Cell 92, 829-839.

Pyle, A., Anugrha, H., Kurzawa-Akanbi, M., Yarnall, A., Burn, D., and Hudson, G. (2015). Reduced mitochondrial DNA copy number is a biomarker of Parkinson's disease. Neurobiol. Aging 38, 216.e7-216.e10.

Raben, N., Plotz, P., and Byrne, B. (2002). Acid a-Glucosidase Deficiency (Glycogenosis Type II, Pompe Disease). Curr. Mol. Med. 2, 145-166.

Raben, N., Wong, A., Ralston, E., and Myerowitz, R. (2012). Autophagy and mitochondria in Pompe disease: Nothing is so new as what has long been forgotten. Am. J. Med. Genet. Part C Semin. Med. Genet. 160 C, 13-21.

Raimundo, N. (2014). Mitochondrial pathology: Stress signals from the energy factory. Trends Mol. Med. 20, 282-292.

Raimundo, N., Baysal, B.E., and Shadel, G.S. (2011). Revisiting the TCA cycle: Signaling to tumor formation. Trends Mol. Med. 17, 641-649.

Raimundo, N., Song, L., Shutt, T.E., McKay, S.E., Cotney, J., Guan, M.X., Gilliland, T.C., Hohuan, D., Santos-Sacchi, J., and Shadel, G.S. (2012). Mitochondrial stress engages E2F1 apoptotic signaling to cause deafness. Cell 148, 716-726.

Raimundo, N., Fern??ndez-Mosquera, L., Yambire, K.F., and Diogo, C. V. (2016). Mechanisms of communication between mitochondria and lysosomes. Int. J. Biochem. Cell Biol. 79, 345-349.

Rapaport, D. (2005). How does the TOM complex mediate insertion of precursor proteins into the mitochondrial outer membrane? J. Cell Biol. 171, 419-423.

Rasola, A., and Bernardi, P. (2011). Mitochondrial permeability transition in Ca2+-dependent apoptosis and necrosis. Cell Calcium 50, 222-233.

Rehling, P., Model, K., Brandner, K., Kovermann, P., Sickmann, A., Meyer, H.E., Kühlbrandt, W., Wagner, R., Truscott, K.N., and Pfanner, N. (2003). Protein Insertion into the 
Mitochondrial Inner Membrane by a Twin-Pore Translocase. Science (80-. ). 299, 1747-1751.

Reichert, A.S., and Neupert, W. (2002). Contact sites between the outer and inner membrane of mitochondria-role in protein transport. Biochim. Biophys. Acta 1592, 41-49.

Rensvold, J.W., Ong, S., Jeevananthan, A., Carr, S.A., Mootha, V.K., and Pagliarini, D.J. (2013). Resource Complementary RNA and Protein Profiling Identifies Iron as a Key Regulator of Mitochondrial Biogenesis. Celrep 3, 237-245.

Rigante, D., Cipolla, C., Basile, U., Gulli, F., and Savastano, M.C. (2017). Overview of immune abnormalities in lysosomal storage disorders. Immunol. Lett. 188, 79-85.

Rink, J., Ghigo, E., Kalaidzidis, Y., and Zerial, M. (2005). Rab conversion as a mechanism of progression from early to late endosomes. Cell 122, 735-749.

Rio, D.C., Ares, M., Hannon, G.J., and Nilsen, T.W. (2010). Purification of RNA using TRIzol (TRI Reagent). Cold Spring Harb. Protoc. 5, 2010-2013.

Rizzuto, R., De Stefani, D., Raffaello, A., and Mammucari, C. (2012). Mitochondria as sensors and regulators of calcium signalling. Nat. Rev. Mol. Cell Biol. 13, 566-578.

Rocha, N., Kuijl, C., Van Der Kant, R., Janssen, L., Houben, D., Janssen, H., Zwart, W., and Neefjes, J. (2009). Cholesterol sensor ORP1L contacts the ER protein VAP to control Rab7RILP-p150Glued and late endosome positioning. J. Cell Biol. 185, 1209-1225.

Roczniak-Ferguson, A., Petit, C.S., Froehlich, F., Qian, S., Ky, J., Angarola, B., Walther, T.C., and Ferguson, S.M. (2012). The Transcription Factor TFEB Links mTORC1 Signaling to Transcriptional Control of Lysosome Homeostasis. Sci. Signal. 5, ra42-ra42.

Rong, Y., Mcphee, C., Deng, S., Chen, L., Liu, M., Tracy, K., Baehreck, E.H., Rong, Y., Mcphee, C.K., Huang, L., et al. (2011). Correction for Rong et al., Spinster is required for autophagic lysosome reformation and mTOR reactivation following starvation. Proc. Natl. Acad. Sci. 108, 11297-11297.

Rong, Y., Liu, M., Ma, L., Du, W., Zhang, H., Tian, Y., Cao, Z., Li, Y., Ren, H., Zhang, C., et al. (2012). Clathrin and phosphatidylinositol-4,5-bisphosphate regulate autophagic lysosome reformation. Nat. Cell Biol. 14, 924-934.

Rosa-Ferreira, C., and Munro, S. (2011). Arl8 and SKIP Act Together to Link Lysosomes to Kinesin-1. Dev. Cell 21, 1171-1178.

Rossignol, R., Gilkerson, R., Aggeler, R., Yamagata, K., Remigton, S.J., and Capaldi, R.A. (2004). Energy substrate modulates mitochondrial structures and oxidative capacityin cancer cells. Cancer Res. 64, 985-993.

Rouault, T.A. (2013). Iron metabolism in the CNS: implications for neurodegenerative diseases. Nat. Rev. Neurosci. 14, 551-564.

Saftig, P., and Klumperman, J. (2009). Lysosome biogenesis and lysosomal membrane proteins: trafficking meets function. Nat. Rev. Mol. Cell Biol. 10, 623-635.

Sakamaki, J. ichi, Wilkinson, S., Hahn, M., Tasdemir, N., O’Prey, J., Clark, W., Hedley, A., Nixon, C., Long, J.S., New, M., et al. (2017). Bromodomain Protein BRD4 Is a Transcriptional Repressor of Autophagy and Lysosomal Function. Mol. Cell 66, 517-532.e9.

Sambri, I., D’Alessio, R., Ezhova, Y., Giuliano, T., Sorrentino, N.C., Cacace, V., De Risi, M., Cataldi, M., Annunziato, L., De Leonibus, E., et al. (2017). Lysosomal dysfunction disrupts 
presynaptic maintenance and restoration of presynaptic function prevents neurodegeneration in lysosomal storage diseases. EMBO Mol. Med. 9, 112-132.

Sato, Y., Kobayashi, H., Higuchi, T., Shimada, Y., Ida, H., and Ohashi, T. (2016). Metabolomic Profiling of Pompe Disease-Induced Pluripotent Stem Cell-Derived Cardiomyocytes Reveals That Oxidative Stress Is Associated With Cardiac and Skeletal Muscle Pathology. Stem Cells Transl. Med. 5, 1-9.

Scalettar, B.A., Abney, J.R., and Hackenbrock, C.R. (1991). Dynamics, structure, and function are coupled in the mitochondrial matrix. Proc. Natl. Acad. Sci. 88, 8057-8061.

Scarpulla, R.C. (2008). Nuclear control of respiratory chain expression by nuclear respiratory factors and PGC-1-related coactivator. Ann. N. Y. Acad. Sci. 1147, 321-334.

Schägger, H., and von Jagow, G. (1991). Blue native electrophoresis for isolation of membrane protein complexes in enzymatically active form. Anal. Biochem. 199, 223-231.

Schägger, H., and Pfeiffer, K. (2000). Supercomplexes in the respiratory chains of yeast and mammalian mitochondria. EMBO J. 19, 1777-1783.

Scheffler, I.E. (2007). Mitochondria: Second Edition.

Schreiber, S.N., Emter, R., Hock, M.B., Knutti, D., Cardenas, J., Podvinec, M., Oakeley, E.J., and Kralli, A. (2004). The estrogen-related receptor alpha (ERRalpha) functions in

PPARgamma coactivator 1alpha (PGC-1alpha)-induced mitochondrial biogenesis. Proc. Natl. Acad. Sci. U. S. A. 101, 6472-6477.

Schuchman, E.H., and Desnick, R.J. (2017). Types A and B Niemann-Pick disease. Mol. Genet. Metab. 120, 27-33.

Schulz, H.N., and Jørgensen, B.B. (2001). Ig acteria. Annu. Rev. Microbiol. 55, 105-137.

Schulze, R.J., Weller, S.G., Schroeder, B., Krueger, E.W., Chi, S., Casey, C.A., and McNiven, M.A. (2013). Lipid droplet breakdown requires dynamin 2 for vesiculation of autolysosomal tubules in hepatocytes. J. Cell Biol. 203, 315-326.

Selak, M.A., Armour, S.M., MacKenzie, E.D., Boulahbel, H., Watson, D.G., Mansfield, K.D., Pan, Y., Simon, M.C., Thompson, C.B., and Gottlieb, E. (2005). Succinate links TCA cycle dysfunction to oncogenesis by inhibiting HIF-a prolyl hydroxylase. Cancer Cell 7, 77-85.

Seth, R.B., Sun, L., Ea, C.K., and Chen, Z.J. (2005). Identification and characterization of MAVS, a mitochondrial antiviral signaling protein that activates NF-kB and IRF3. Cell 122, 669-682.

Settembre, C., Polito, V.A., Garcia, M., Vetrini, F., Erdin, S., Erdin, S.U., Huynh, T., Medina, D., Colella, P., Sardiello, M., et al. (2011). TFEB Links Autophagy to Lysosomal Biogenesis. Science (80-. ). 332, 1429-1433.

Settembre, C., Zoncu, R., Medina, D.L., Vetrini, F., Erdin, S., Erdin, S., Huynh, T., Ferron, M., Karsenty, G., Vellard, M.C., et al. (2012). A lysosome-to-nucleus signalling mechanism senses and regulates the lysosome via mTOR and TFEB. EMBO J. 31, 1095-1108.

Shadel, G.S., and Clayton, D.A. (1997). Mitochondrial DNA maintenance in vertebrates. Annu. Rev. Biochem. 66, 409-435.

Shen, H.M., and Mizushima, N. (2014). At the end of the autophagic road: An emerging understanding of lysosomal functions in autophagy. Trends Biochem. Sci. 39, 61-71. 
Sherman, L.E., Go, N.E., and Nargang, F.E. (2005). Functions of the Small Proteins in the TOM Complex of Neurospora crasssa. Mol. Biol. Cell 16, 4172-4182.

Sinclair, L. V, Finlay, D., Feijoo, C., Cornish, G.H., Gray, A., Ager, A., Okkenhaug, K., Hagenbeek, T.J., Spits, H., and Cantrell, D. a (2008). Phosphatidylinositol-3-OH kinase and nutrient-sensing mTOR pathways control T lymphocyte trafficking. Nat. Immunol. 9, 513-521.

Skon, C.N., Lee, J.-Y., Anderson, K.G., Masopust, D., Hogquist, K.A., and Jameson, S.C. (2013). Transcriptional downregulation of S1pr1 is required for the establishment of resident memory CD8+ T cells. Nat. Immunol. 14, 1285-1293.

Smirnova, E., Griparic, L., Shurland, D.-L., and Bliek, A.M. van der (2001). Dynamin-related Protein Drp1 Is Required for Mitochondrial Division in Mammalian Cells. Mol. Biol. Cell 12, 2245-2256.

Song, Z., Ghochani, M., McCaffery, M.J., Frey, T.G., and Chan, D.C. (2009). Mitofusins and OPA1 mediate sequential steps in mitochondrial membrane fusion. Mol. Biol. Cell 20, 35253532.

Soubannier, V., McLelland, G.L., Zunino, R., Braschi, E., Rippstein, P., Fon, E.A., and McBride, H.M. (2012). A vesicular transport pathway shuttles cargo from mitochondria to lysosomes. Curr. Biol. 22, 135-141.

Staretz-Chacham, O., Lang, T.C., LaMarca, M.E., Krasnewich, D., and Sidransky, E. (2009). Lysosomal Storage Disorders in the Newborn. Pediatrics 123, 1191-1207.

Stiller, S.B., Höpker, J., Oeljeklaus, S., Schütze, C., Schrempp, S.G., Vent-Schmidt, J., Horvath, S.E., Frazier, A.E., Gebert, N., Van Der Laan, M., et al. (2016). Mitochondrial OXA Translocase Plays a Major Role in Biogenesis of Inner-Membrane Proteins. Cell Metab. 23, 901-908.

Stojanovski, D., Müller, J.M., Milenkovic, D., Guiard, B., Pfanner, N., and Chacinska, A. (2008). The MIA system for protein import into the mitochondrial intermembrane space. Biochim. Biophys. Acta - Mol. Cell Res. 1783, 610-617.

Tufi, R., Gandhi, S., de Castro, I.P., Lehmann, S., Angelova, P.R., Dinsdale, D., Deas, E., Plun-Favreau, H., Nicotera, P., Abramov, A.Y., et al. (2014). Enhancing nucleotide metabolism protects against mitochondrial dysfunction and neurodegeneration in a PINK1 model of Parkinson's disease. Nat Cell Biol 16, 157-166.

Turner, S., Falk, D., and Byrne, B. (2016). Transcriptome assessment of the Pompe (Gaa-/-) mouse spinal cord indicates widespread neuropathology. Physiological 1012, 785-794.

Vanier, M.T. (2013). Niemann-Pick diseases (Elsevier B.V.).

Varlamov, D. a, Kudin, A.P., Vielhaber, S., Schröder, R., Sassen, R., Becker, A., Kunz, D., Haug, K., Rebstock, J., Heils, A., et al. (2002). Metabolic consequences of a novel missense mutation of the mtDNA CO I gene. Hum. Mol. Genet. 11, 1797-1805.

Virbasius, C.M.A., Virbasius, J. V., and Scarpulla, R.C. (1993a). NRF-1, an activator involved in nuclear-mitochondrial interactions, utilizes a new DNA-binding domain conserved in a family of developmental regulators. Genes Dev. 7, 2431-2445.

Virbasius, J. V., Virbasius, C.A., and Scarpulla, R.C. (1993b). Identity of GABP with NRF-2, a multisubunit activator of cytochrome oxidase expression, reveals a cellular role for and ETS domain activator of viral promoters. Genes Dev. 7, 380-392. 
Vitner, E.B., Futerman, A.H., and Platt, N. (2015). Innate immune responses in the brain of sphingolipid lysosomal storage diseases. Biol. Chem. 396, 659-667.

Te Vruchte, D., Lloyd-Evans, E., Veldman, R.J., Neville, D.C.A., Dwek, R.A., Platt, F.M., Van Blitterswijk, W.J., and Sillences, D.J. (2004). Accumulation of glycosphingolipids in NiemannPick C disease disrupts endosomal transport. J. Biol. Chem. 279, 26167-26175.

Watanabe, T., Frahm, J., and Michaelis, T. (2015). In Vivo Brain MR Imaging at Subnanoliter Resolution: Contrast and Histology. Magn. Reson. Med. Sci. 15, 11-25.

Watanabe, T., Frahm, J., and Michaelis, T. (2016). Amide proton signals as pH indicator for in vivo MRS and MRI of the brain-Responses to hypercapnia and hypothermia. Neuroimage 133, 390-398.

West, A.P., and Shadel, G.S. (2017). Mitochondrial DNA in innate immune responses and inflammatory pathology. Nat. Rev. Immunol. 17, 363-375.

West, A.P., Shadel, G.S., and Ghosh, S. (2011). Mitochondria in innate immune responses. Nat. Rev. Immunol. 11, 389-402.

West, A.P., Khoury-Hanold, W., Staron, M., Tal, M.C., Pineda, C.M., Lang, S.M., Bestwick, M., Duguay, B.A., Raimundo, N., MacDuff, D.A., et al. (2015). Mitochondrial DNA stress primes the antiviral innate immune response. Nature 520, 553-557.

Whitworth, A.J., and Pallanck, L.J. (2017). PINK1/Parkin mitophagy and neurodegenerationwhat do we really know in vivo? Curr. Opin. Genet. Dev. 44, 47-53.

Wiedemann, N., Kozjak, V., Chacinska, A., Schoenfisch, B., Rospert, S., Ryan, M.T., Pfanner, N., and Meisinger, C. (2003). Machinery for protein sorting and assembly in the mitochondrial outer membrane. Nature 424, 565-571.

Wiedemann, N., Frazier, A.E., and Pfanner, N. (2004). The Protein Import Machinery of Mitochondria. J. Biol. Chem. 279, 14473-14476.

Wiese, S., Gronemeyer, T., Ofman, R., Kunze, M., Grou, C.P., Almeida, J.A., Eisenacher, M., Stephan, C., Hayen, H., Schollenberger, L., et al. (2007). Proteomics Characterization of Mouse Kidney Peroxisomes by Tandem Mass Spectrometry and Protein Correlation Profiling. Mol. Cell. Proteomics 6, 2045-2057.

Winterbourn, C.C. (1995). Toxicity of iron and hydrogen peroxide: the Fenton reaction. Toxicol. Lett. 82-83, 969-974.

Wong, T.W., and Clayton, D.A. (1985). In vitro replication of human mitochondrial DNA: Accurate initiation at the origin of light-strand synthesis. Cell 42, 951-958.

Woś, M., Szczepanowska, J., Pikuła, S., Tylki-Szymańska, A., Zabłocki, K., and Bandorowicz-Pikuła, J. (2016). Mitochondrial dysfunction in fibroblasts derived from patients with Niemann-Pick type C disease. Arch. Biochem. Biophys. 593, 50-59.

Xu, L.G., Wang, Y.Y., Han, K.J., Li, L.Y., Zhai, Z., and Shu, H.B. (2005). VISA is an adapter protein required for virus-triggered IFN-?? signaling. Mol. Cell 19, 727-740.

Xu, S., Zhong, M., Zhang, L., Wang, Y., Zhou, Z., Hao, Y., Zhang, W., Yang, X., Wei, A., Pei, L., et al. (2009). Overexpression of Tfam protects mitochondria against ??-amyloid-induced oxidative damage in SH-SY5Y cells. FEBS J. 276, 4224-4233.

Yao, L., Kan, E.M., Lu, J., Hao, A., Dheen, S.T., Kaur, C., and Ling, E.A. (2013). Toll-like 
receptor 4 mediates microglial activation and production of inflammatory mediators in neonatal rat brain following hypoxia: role of TLR4 in hypoxic microglia. J Neuroinflammation $10,23$.

Yasukawa, T., Reyes, A., Cluett, T.J., Yang, M.-Y., Bowmaker, M., Jacobs, H.T., and Holt, I.J. (2006). Replication of vertebrate mitochondrial DNA entails transient ribonucleotide incorporation throughout the lagging strand. EMBO J. 25, 5358-5371.

Youle, R.J., and Narendra, D.P. (2011). Mechanisms of mitophagy. Nat. Rev. Mol. Cell Biol. 12, 9-14.

Young, J.C., Hoogenraad, N.J., and Hartl, F.U. (2003). Molecular chaperones Hsp90 and Hsp70 deliver preproteins to the mitochondrial import receptor Tom70. Cell 112, 41-50.

Yu, L., McPhee, C.K., Zheng, L., Mardones, G.A., Rong, Y., Peng, J., Mi, N., Zhao, Y., Liu, Z., Wan, F., et al. (2010). Termination of autophagy and reformation of lysosomes regulated by mTOR. Nature $465,942-946$.

Zalman, L.S., Nikaido, H., and Kagawa, Y. (1980). Mitochondrial outer membrane contains a protein producing nonspecific diffusion channels. J. Biol. Chem. 255, 1771-1774.

Zeigerer, A., Gilleron, J., Bogorad, R.L., Marsico, G., Nonaka, H., Seifert, S., Epstein-Barash, H., Kuchimanchi, S., Peng, C.G., Ruda, V.M., et al. (2012). Rab5 is necessary for the biogenesis of the endolysosomal system in vivo. Nature 485, 465-470.

Zhang, Q., Raoof, M., Chen, Y., Sumi, Y., Sursal, T., Junger, W., Brohi, K., Itagaki, K., and Hauser, C.J. (2010). Circulating mitochondrial DAMPs cause inflammatory responses to injury. Nature 464, 104-107.

Zhao, M., Antunes, F., Eaton, J.W., and Brunk, U.T. (2003). Lysosomal enzymes promote mitochondrial oxidant production, cytochrome c release and apoptosis. Eur. J. Biochem. 270, 3778-3786. 


\section{Appendix}

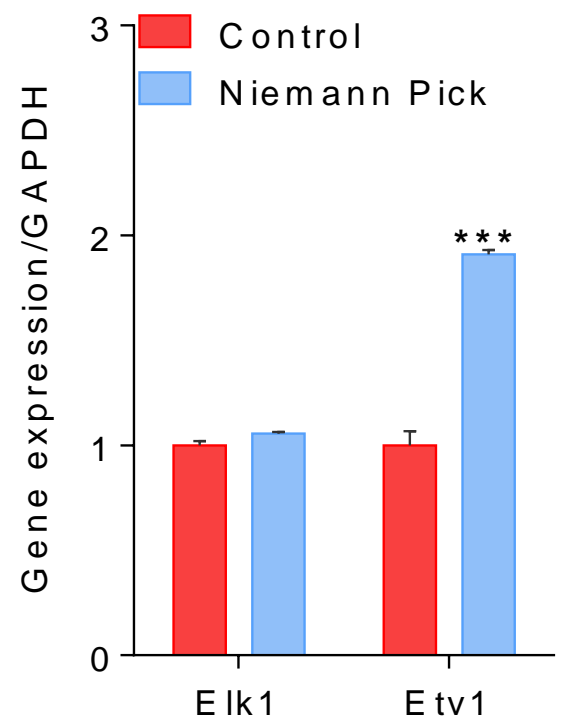

Figure 52| Expression levels of ETS transcription factors in NP patient cells Transcript levels of ETV1 are increased by about two-fold in NP fibroblasts while ELK1 levels are unchanged. $\mathrm{p}<0.001\left(^{* * *}\right)$; Student's t-test; mean \pm SEM, N = 8; SEM: standard error of the mean

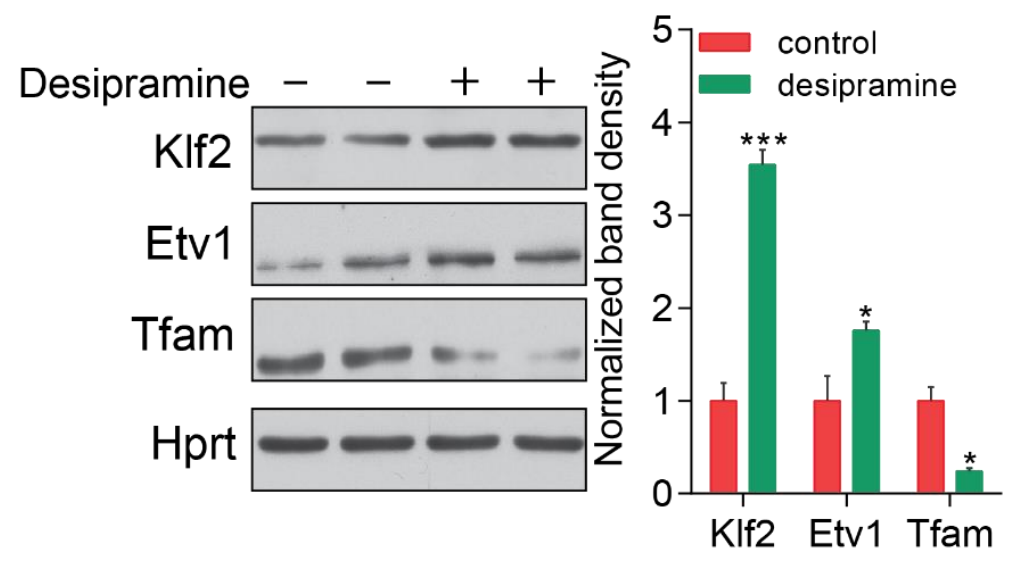

Figure 53| KLF2, ETV1 and TFAM protein levels in desipramine-treated cells Desipramine-treated human fibroblasts, like NP fibroblasts, showed increased KLF2 and ETV1 protein levels, and reduced TFAM protein levels. Graphs are mean $\pm \mathrm{SD}, \mathrm{N}=3 ; \mathrm{p}<0.05\left(^{*}\right)$ and $\mathrm{p}<0.00\left(^{* * *}\right)$; Student's t-test; SD: standard deviation 


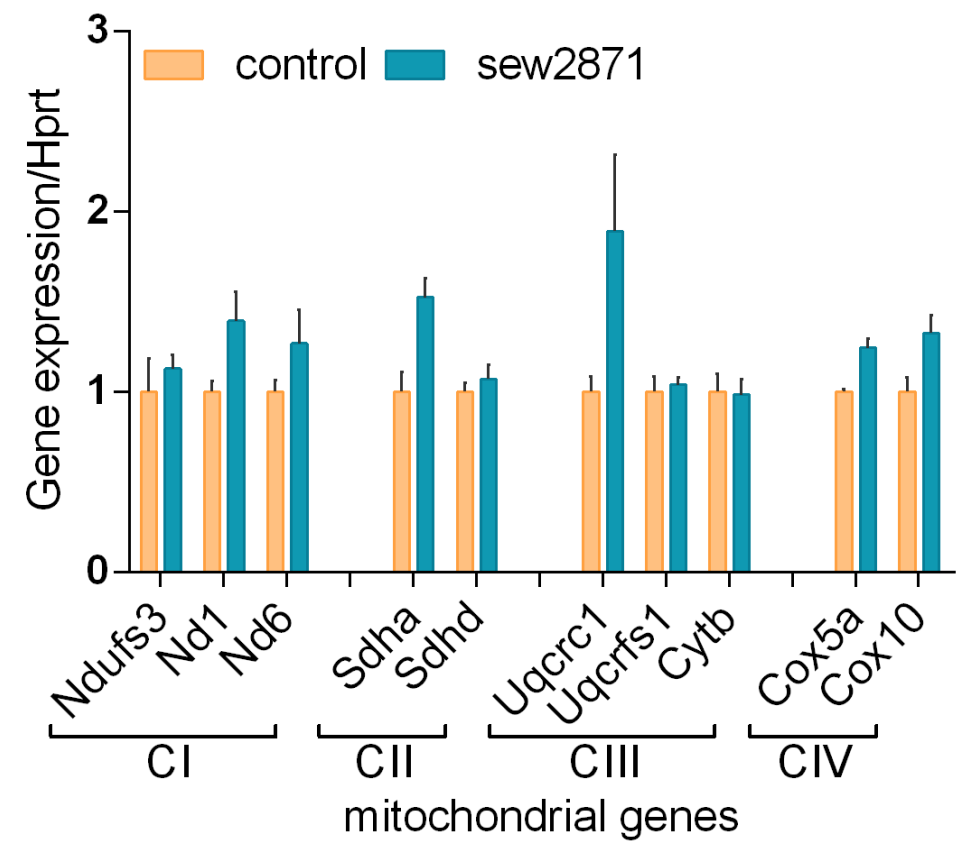

Figure 54| Enhanced S1P signaling induces mitochondrial gene expression Treatment of control human fibroblasts with Sew2871 (S1P receptor agonist) results in increased mitochondrial transcript levels.

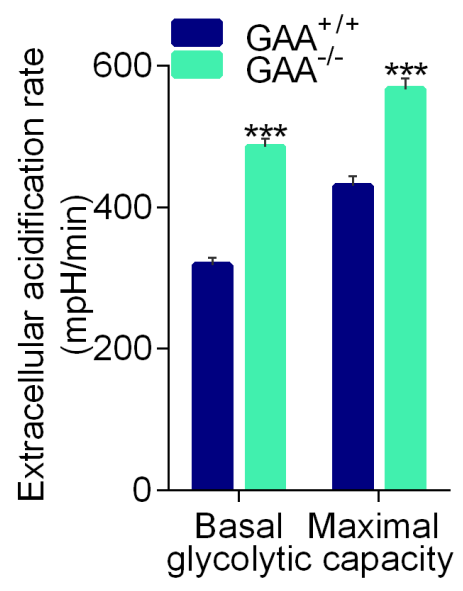

Figure 55| Increased glycolysis in GAA /- MEFs - Extracellular acidification rate measurements showed increased basal and maximal glycolytic capacities in GAA $\%$ MEFs. p < $\left.0.001{ }^{* * *}\right)$; Student's t-test; mean \pm SEM, N = 8; SEM: standard error of the mean 


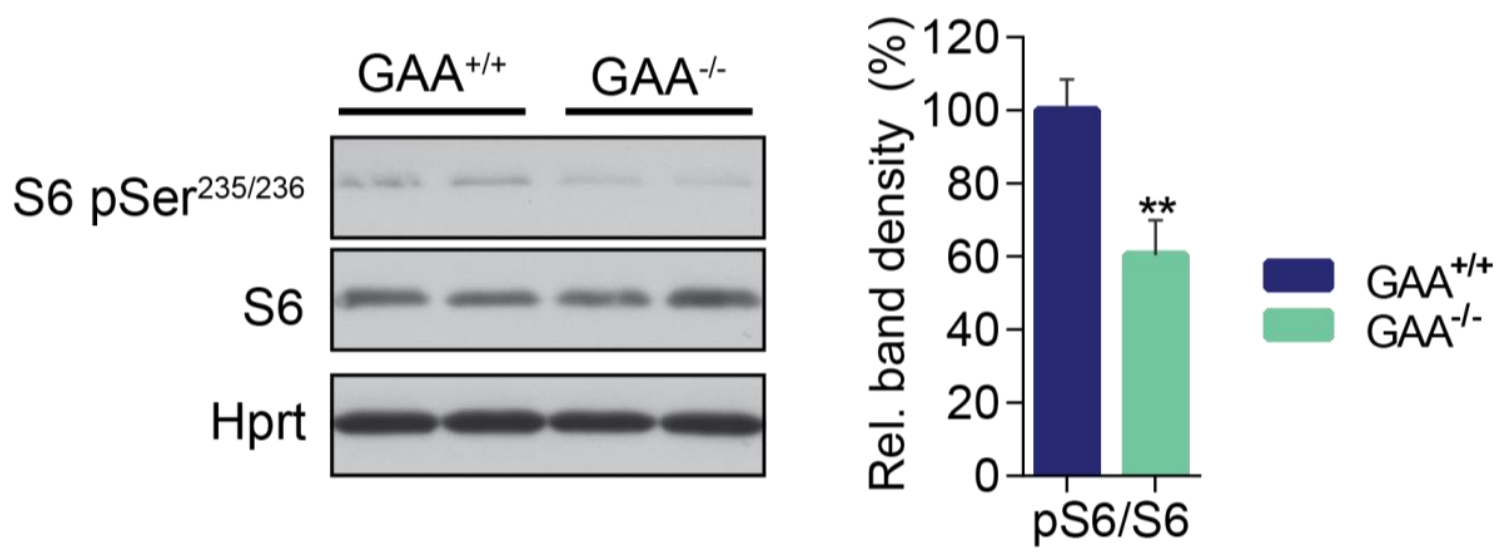

Figure 56| mTORC1 activity in GAA /- cortex - mTORC1 activity in the cortex of GAA / mice is reduced. This is demonstrated by decreased phosphorylation of ribosomal protein S6 at Serine 235/236. Band quantifications are represented as mean $\pm \mathrm{SEM}, \mathrm{N}=8$; $\mathrm{p}<0.01\left(^{* *}\right.$ ); Student's t-test; SEM: standard error of the mean
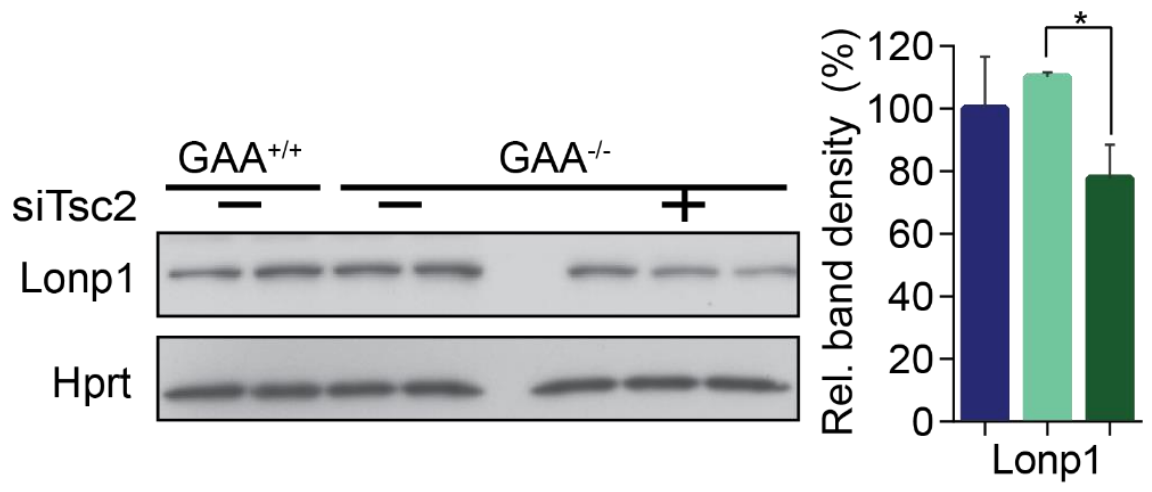

$\mathrm{GAA}^{+/+}$siCtrl

$\mathrm{GAA}^{-/-}$siCtrl

$\mathrm{GAA}^{-/-} \mathrm{siTsc} 2$

Figure 57| LONP1 levels are partly dependent on mTORC1 activity in GAA /- MEFs

- LONP1 protein levels in GAA \% MEFs are partially reduced following siTSC2-mediated induction of mTORC1 activity. Band quantifications are depicted as mean \pm SD for triplicate samples; $\mathrm{p}<0.05\left(^{*}\right)$; Student's t-test; SD: standard deviation 

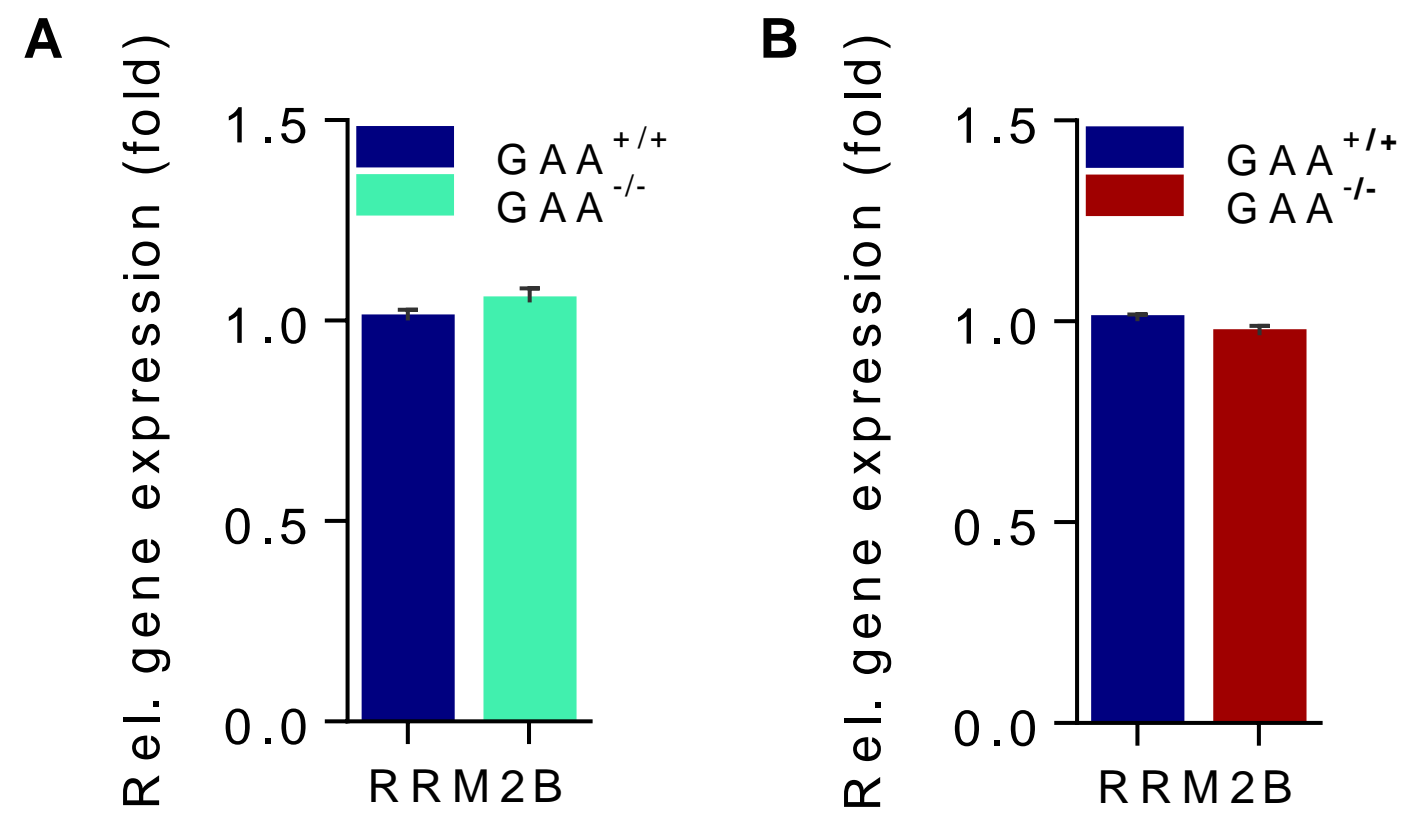

Figure 58| RRM2B gene expression in GAA-/- mice - RRM2B transcript levels in the cortex (A) and in the heart (B) of $\mathrm{GAA}^{-/}$mice. Gene expression levels of RRM2B are unchanged based on $\mathrm{p}>0.05$; Student's t-test. Graphs represent mean \pm SEM, N = 8; SEM: standard error of the mean 


\section{List of publications}

Diogo CV, Yambire KF, Fernandez-Mosquera L, Branco T and Raimundo N (2017) Mitochondrial adventures at the organelle society BBRC: 1-7.

Fernandez-Mosquera L, Diogo CV, Yambire KF, Santos GL, ...., and Raimundo N (2017) Acute and chronic respiratory chain deficiency differentially regulate lysosomal biogenesis. Scientific Reports $(7): 1-11$

Raimundo N, Fernandez-Mosquera L, Yambire KF and Diogo CV (2016) Mechanisms of communication between mitochondria and lysosomes. Int J Biochem Cell Biol (79): 345-349.

Nuno Raimundo, Lorena Fernandez-Mosquera, and King Faisal Yambire (2015) Mitochondrial Signaling. In: Hockenbery, D e.d. Mitochondrial and Cell Death Springer

\section{Manuscript(s) in revision}

Yambire KF, Fernandez-Mosquera L, Steinfeld R, Ikonen E, Milosevic I and Raimundo N (2017) Impaired sphingosine-1-phosphate signaling in Niemann-Pick disease triggers repression of mitochondrial biogenesis via KLF2 and ETV1 PLoS Biology: In revision

\section{Manuscript(s) in preparation}

Yambire KF, Rostosky C, Watanabe T, Pacheu-Grau D,...., Milosevic I and Raimundo N (2017) Perturbed iron homeostasis links lysosomal malfunction to mtDNA depletion and induction of the innate immune response. In preparation.

Fernandez-Mousquera L, Yambire KF, Diogo CV,...., and Raimundo N (2017) Mitochondrial deficiency impairs lysosomal function via AMPK-PIKFYVE-PI(3,5) $\mathrm{P}_{2}-M C O L N 1$ pathway. In preparation. 


\section{Acknowledgements}

This work was carried out in the labs of Dr. Nuno Raimundo (Institute of Cellular Biochemistry) and Dr. Ira Milosevic (European Neuroscience Institute), who both mentored me through the past four years. I am very grateful for the unwavering support and opportunity you provided me throughout my PhD studies. Mostly staying in the middle, I have learned a great deal from both of you, about how to approach science from different perspectives. To Nuno, we discussed science whenever we wanted; even at times when you were away, in the 'remotest' of places without good access to internet. We even discussed football from Benfica through Real Madrid to Arsene and Mourinho and it was engaging. To Ira, our discussions were always fruitful since you constantly pointed out the essential details of what needed to be done. You always wanted me to succeed and did everything possible to ensure this. When I joined your labs, I witnessed, from first-hand, the challenges of starting a lab and striving for greater success in the midst of those challenges. You both never hesitated, even for once, to provide me with the needed resources for my work during these years and I am eternally grateful for that. Two heads, they say, are indeed better than one! You have both me made me a better scientist.

I am thankful for the support and helpful discussions of Prof. André Fischer and Prof. KlausArmin Nave, who were part of my thesis committee. You both stimulated my interest in science, and guided me through the right paths in the last years. I would like to thank Prof. Steven Johnsen for the immense support as a member of the examination board for my thesis defense, and for your useful discussions and help with appropriate cell lines for my project.

Every adventure requires a first step and mine was the MSc/PhD Neuroscience program, which granted me financial support in the Master's phase of my studies. My sincere gratitude goes to Prof. Michael Hörner and Sandra Drube of the coordination office for your continuous guidance and support since my first day in Germany. You always cared for me, listened to my challenges and offered solutions to most of my problems. Danke Ihnen!

I would like to acknowledge the assistance of the diligent collaborators I worked and discussed with over the years on several projects including Dr. David Pacheu-Grau, Dr. Takashi Watanabe, Dr. Massimiliano Stagi, and Prof. Phillip West. Thank you all!

To all past and present members of the Raimundo and Milosevic labs, I say thank you for your support and for providing the enabling environment to carry out this work. I am especially grateful to Christine, Sindhu, Lorena, Leonardo and Renata for your experimental support and meaningful discussions. 
I am grateful to Dr. Anita Krisko, Dr. John Murdoch, Dr. Lorena Fernandez-Mosquera, Sindhu, and Isaac for taking time off your busy schedule to peruse this thesis.

I made friends along the way, lost some, and some stayed on and even became 'family'. You have been with me through several winters and tolerated my troubles. I am especially thankful to Sindhu, Lorena, Isaac, Leonardo, Anita, Peter and Jonida.

Distance means so little when you mean so much to me. I am thankful to my family, especially my mum and dad for the continuous support and love. I am grateful to Uncle Samuel for been a great mentor and for his support throughout my career. You all spur me on! 